\title{
WestVirginiaUniversity
}

THE RESEARCH REPOSITORY @ WVU

Graduate Theses, Dissertations, and Problem Reports

2013

\section{Modeling of High Pressure Confined Inflatable Structures}

Jerry C. Wong

Follow this and additional works at: https://researchrepository.wvu.edu/etd

\section{Recommended Citation}

Wong, Jerry C., "Modeling of High Pressure Confined Inflatable Structures" (2013). Graduate Theses, Dissertations, and Problem Reports. 7357.

https://researchrepository.wvu.edu/etd/7357

This Dissertation is protected by copyright and/or related rights. It has been brought to you by the The Research Repository @ WVU with permission from the rights-holder(s). You are free to use this Dissertation in any way that is permitted by the copyright and related rights legislation that applies to your use. For other uses you must obtain permission from the rights-holder(s) directly, unless additional rights are indicated by a Creative Commons license in the record and/ or on the work itself. This Dissertation has been accepted for inclusion in WVU Graduate Theses, Dissertations, and Problem Reports collection by an authorized administrator of The Research Repository @ WVU.

For more information, please contact researchrepository@mail.wvu.edu. 
Modeling of High Pressure Confined Inflatable Structures

Jerry C Wong

\begin{abstract}
Dissertation submitted to the
Benjamin M. Statler College of Engineering and Mineral Resources at West Virginia University in partial fulfillment of the requirements for the degree of
\end{abstract}

\author{
Doctor of Philosophy \\ in \\ Mechanical Engineering
}

Department of Mechanical and Aerospace Engineering

Dr. Ever J. Barbero, Chair

Dr. Eduardo M. Sosa, Co-Chair

Dr. Victor H. Mucino

Dr. Jacky C. Prucz

Dr. Xingbo Liu

\title{
Morgantown, West Virginia \\ 2013
}

Keywords: inflatable structure, finite element analysis, airbag deployment, tunnel safety Copyright (C) 2013 Jerry C. Wong 
UMI Number: 3605890

All rights reserved

INFORMATION TO ALL USERS

The quality of this reproduction is dependent upon the quality of the copy submitted.

In the unlikely event that the author did not send a complete manuscript and there are missing pages, these will be noted. Also, if material had to be removed, a note will indicate the deletion.

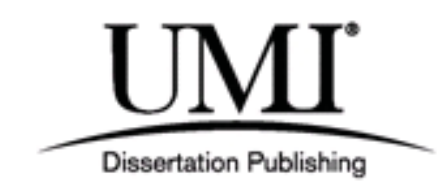

UMI 3605890

Published by ProQuest LLC (2013). Copyright in the Dissertation held by the Author.

Microform Edition (C) ProQuest LLC.

All rights reserved. This work is protected against unauthorized copying under Title 17, United States Code

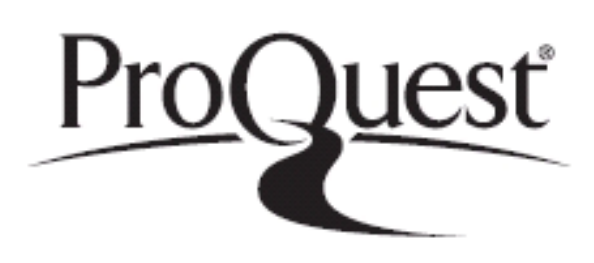

ProQuest LLC.

789 East Eisenhower Parkway

P.O. Box 1346

Ann Arbor, MI 48106 - 1346 


\section{Abstract \\ Modeling of High Pressure Confined Inflatable Structures \\ Jerry C Wong}

Safety of transportation tunnels is a top priority among transportation agencies and public administrators and a very important aspect in the daily operation of a tunnel system. However, it is always a challenge to create and integrate protection systems in existing tunnels to prevent or at least mitigate the occurrence of hazardous events such as spread of smoke or noxious fumes, flooding, among others. Typically there two ways for preventing or mitigating the occurrence of hazardous events: one is the implementation of permanent solutions and, the second one, is the use of temporary solutions. Permanent solutions usually have relatively high sealing efficiency due to their solid and rigid sealing mechanisms such as bulkheads and floodgates. However, they can be extremely expensive and sometimes difficult to build or install due to physical, economical or operational constrains. On the other hand, temporary solutions, which can be relatively low cost and easy to install, offer a temporary countermeasure while permanent repairs are implemented. The development of flexible structures, such as inflatable plugs for temporary solutions is becoming a viable alternative for protection of transportation tunnels and other similar critical civil infrastructure.

The Resilient Tunnel System (RTS) is a passive tunnel protection system developed at West Virginia University (WVU). This system is intended to prevent or minimize the damage induced by hazardous events by creating a compartment to contain the threat. The Resilient Tunnel System implements inflatable structures at specific locations of the tunnel to seal up the tunnel and create a compartment to isolate the compromised region. WVU has conducted several validation tests on full scale inflatable structures designed to mitigate flooding in an actual rail transportation tunnel and in specially built testing facilities. However, testing at full scale either in an actual tunnel or in specially built testing facilities, is a very complex and resource demanding task. It can take several iterations to achieve desired results which cannot be accurately 
predicted in advance. Therefore, the development of numerical models using Finite Element Analysis becomes imperative in order to: first, reproduce experimental work done at WVU using different prototypes at different scales; and then use the calibrated models as predicting tool that can anticipate the outcome of experiments and eventually reduce its number due to the intrinsic complexity and cost.

This dissertation aims to present the results of the development of Finite Element Models of confined inflatable structures designed to withstand flooding pressures. Models of different prototypes were created and analyzed in order to reproduce experimental results. Numerical results show that the adjusted models can reproduce experimental results, ranging from deployment, full pressurization and induced failure, with a great degree of accuracy providing a reliable predicting tool for evaluation of alternative configurations and parametric studies. 
To my wife, Sophia Wong, and my daughters, Emma Wong and Anna Wong. 


\section{Acknowledgements}

First of all, I would like to praise the Lord for going through every difficult moment with me and putting a great number of good people to help me during the entire journey.

I would like to show my greatest gratitude my research advisor and mentor, Dr. Ever Barbero, who gave me opportunities to work on short projects when I started my doctoral degree and a doctoral research opportunity later when I needed one. His guidance, support, and encouragement have made this a wonderful journey.

Dr. Eduardo Sosa has been an incredible research advisor who spent countless hours on checking my work and proofreading. “Jerry, don't give up! We are almost there.", Dr. Sosa always said this to me whenever I was overwhelmed by the research. The same encouraging phrase keeps coming up in my mind whenever I think of my dissertation. Dr. Sosa's encouragement and support have motivated me through the entire journey, especially during the final stage of writing.

Dr. Victor Mucino is one of my dissertation committees. He provided assistantship and allowed flexible working hours, so that I could focus on writing. This dissertation would never be the same without his support. I also would like to thank my dissertation committees of Dr. Jacky Prucz, and Dr. Xingbo Liu for their support for over the past four years.

I also would like to thank Mr. Bartosz Gradzik for his generosity in sharing his knowledge of scripting, coding, as well as his experience in modeling. Mr. Gradzik also shared his time selflessly in teaching me modeling techniques. I really appreciate the extra knowledge that Mr. Gradzik has added on me as well as our friendship.

I would like to share this honor with my parents who always show their unconditional love to their only son. My parents-in-law took care of Sophia and Emma when I started this dissertation and I really appreciate their help. Pastor Joseph Cheng and Mrs. Sharon Cheng also gave a hand to Sophia and I by taking care of Emma while Sophia was delivering Anna in the hospital. 
Last but not least, I would like to thank my sisters, Foon Ngok, Hoon King, and Siaw Foong and my brother-in-law, Wan Ran, for their support. 


\section{Table of Contents}

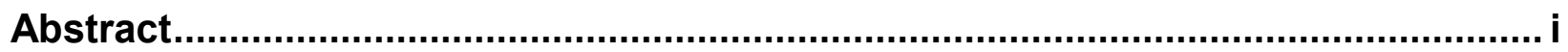

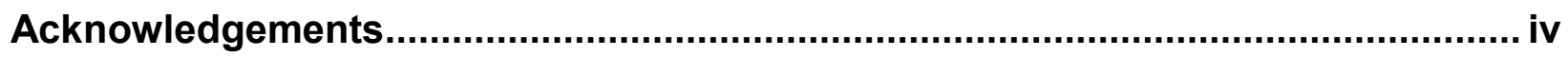

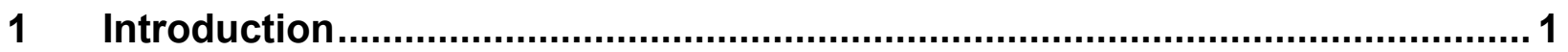

1.1 Origin, Evolution, and Applications of Inflatable Structures....................... 1

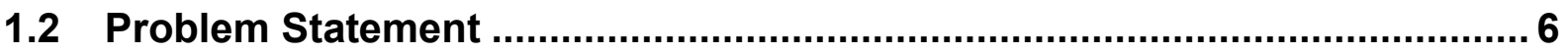

1.3 Resilient Tunnel Plug (RTP) System ...................................................... 9

1.4 Advantages and Disadvantages of Inflatable Structures for Tunnel

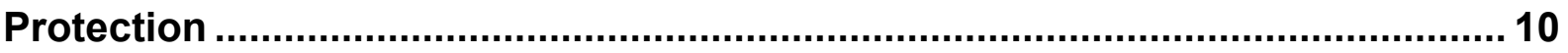

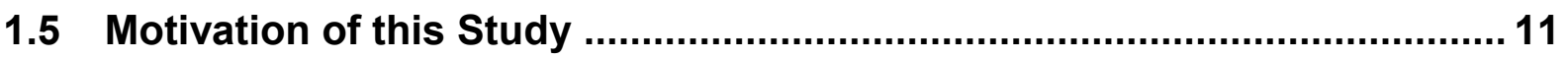

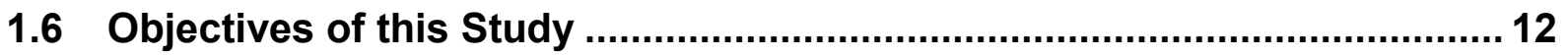

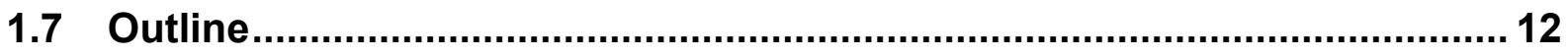

2 Introduction to the Finite Element Method ...................................................... 14

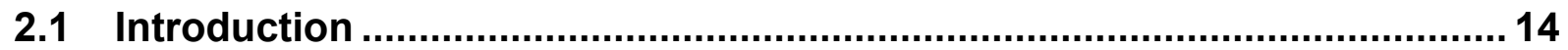

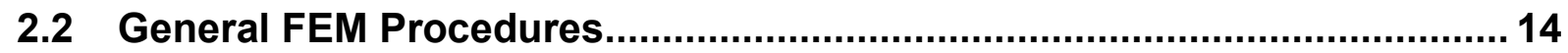

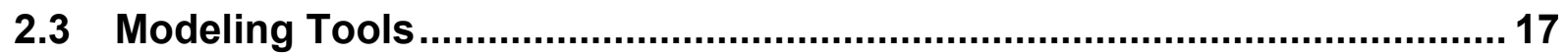

2.3.1 Element Types ................................................................................... 17

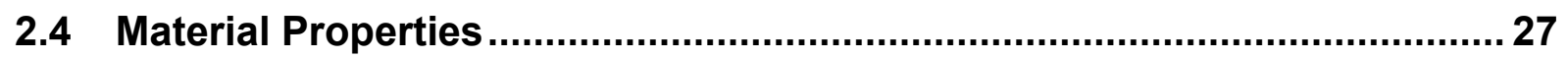

2.4.1 Fabric Material Behavior .............................................................. 28

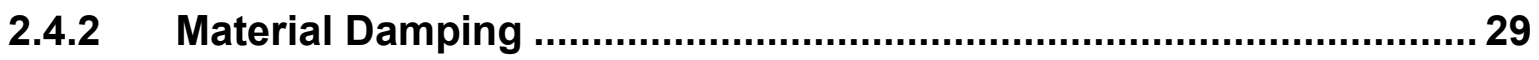

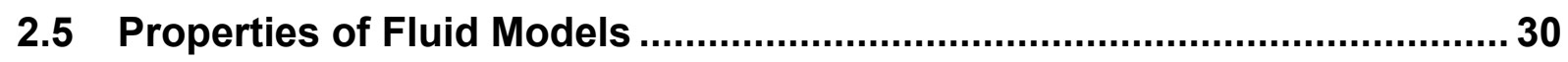

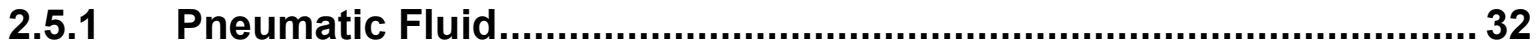

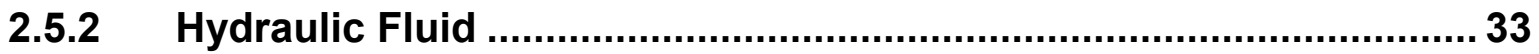

2.5.3 Surface-based Fluid Cavity Algorithm ............................................ 33

2.6 Friction and Contact Properties ............................................................ 37 


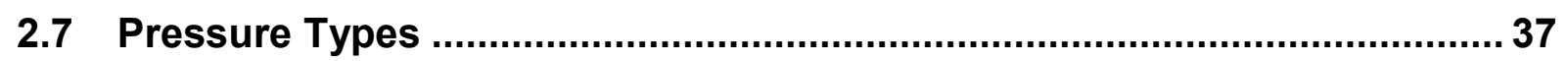

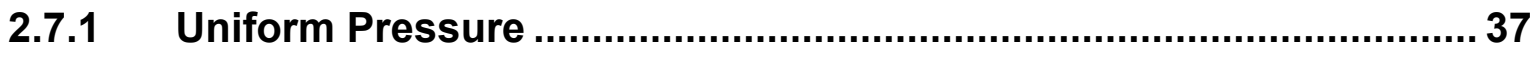

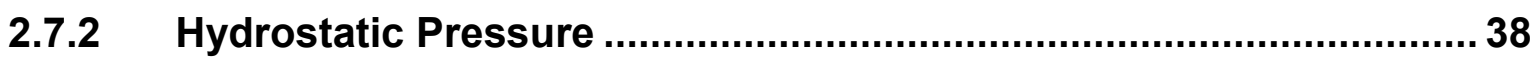

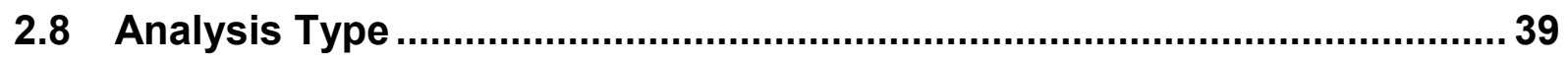

2.8.1 Explicit Dynamic Analysis ............................................................ 39

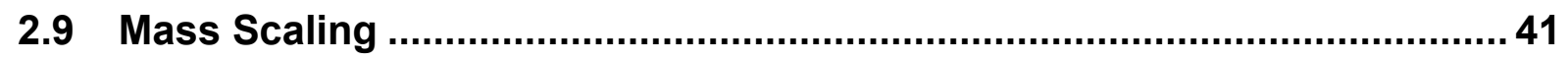

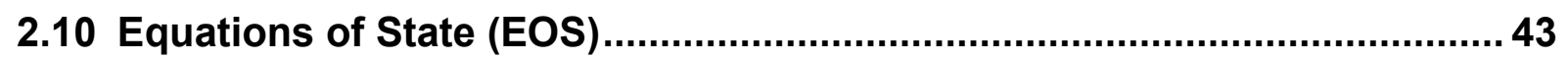

3 Reduced-Scale Prototype: Model Evaluation .................................................. 47

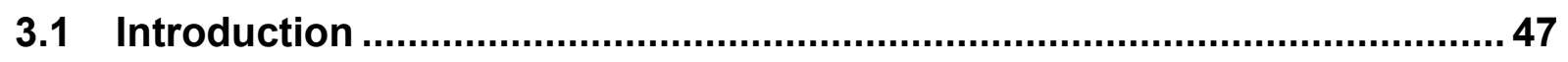

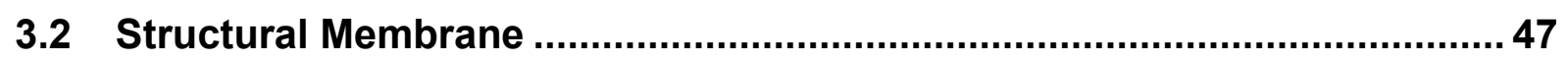

3.3 Assumptions for Modeling of Reduced-Scale Prototype ..........................49

3.4 Modeling Plan of Reduced-Scale Prototype .............................................49

3.5 FE Model Set-up of Reduced-Scale Prototype ........................................ 51

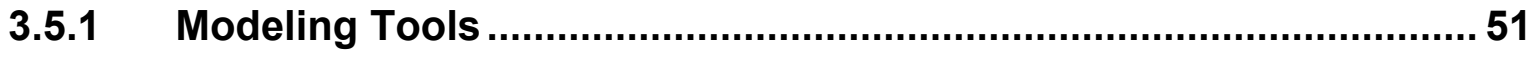

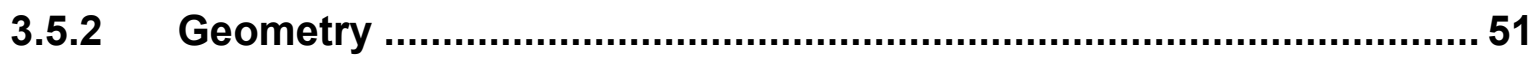

3.5.3 Element Selection and Meshing..................................................52

3.6 Modeling of Simplified Deployment of Reduced-Scale Prototype .............. 54

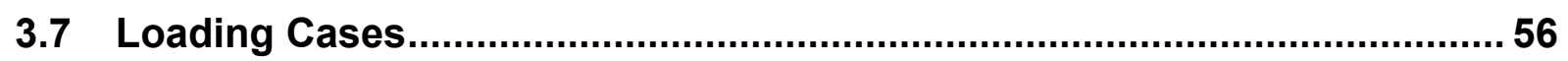

3.7.1 Loading Case I: Stress Evaluation.....................................................56

3.7.2 Loading Case II: Elongation Evaluation ........................................ 57

3.7.3 Loading Case III: Axial Slippage Evaluation ......................................60

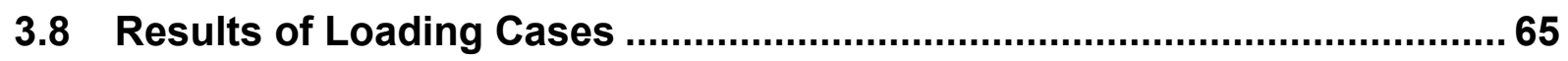

3.8.1 Loading Case I: Stress Evaluation........................................................ 65

3.8.2 Loading Case II: Elongation Evaluation .......................................... 66

3.8.3 Loading Case III: Axial Slippage Evaluation .....................................70 


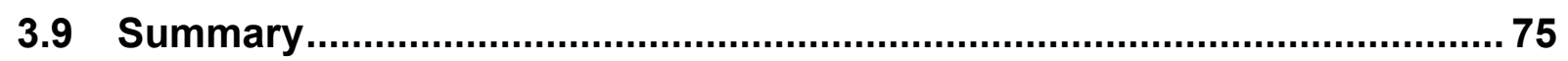

$4 \quad$ Full-Scale Prototype: FE Model Initial Preparation ............................................. 77

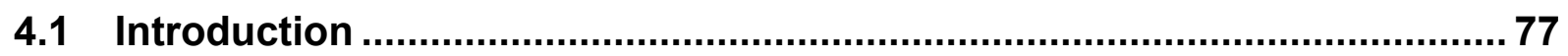

4.2 Dimensions of Full-Scale Prototype …................................................... 77

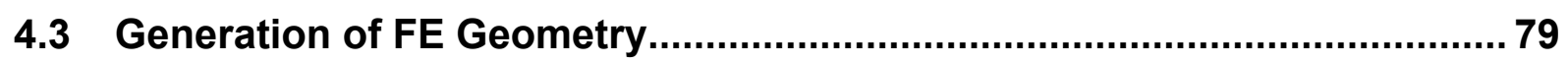

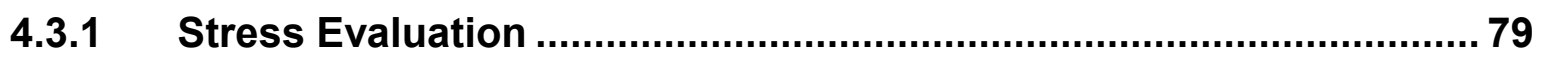

4.3.2 Mesh Convergence Study ............................................................ 82

4.4 Geometries of Tunnel, Base, and Rotational Plates. ................................. 83

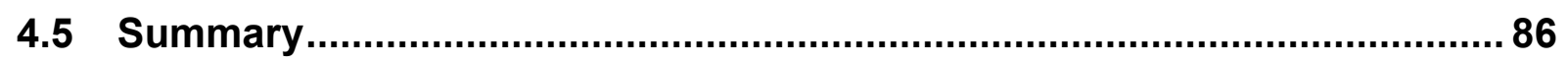

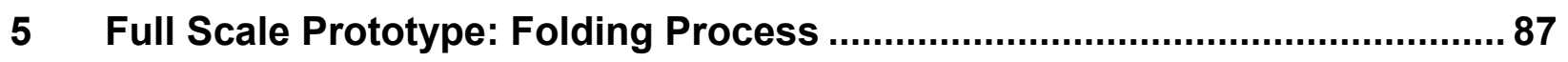

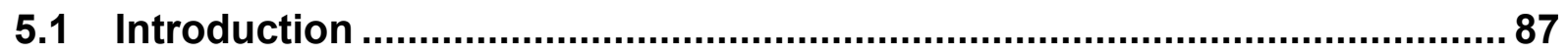

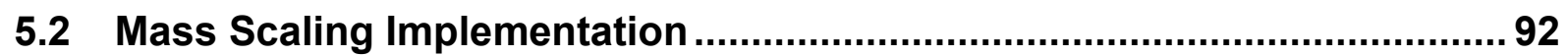

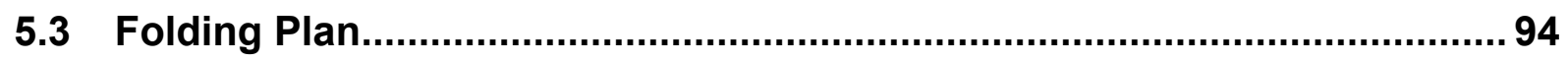

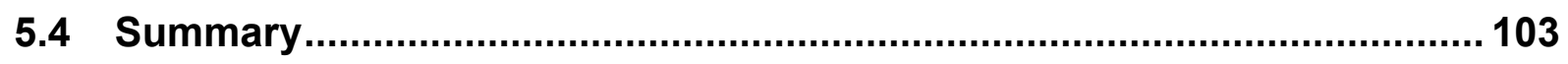

6 Full-Scale Prototype: Placement and Dynamic Relaxation............................ 104

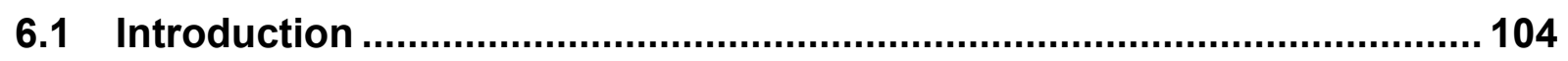

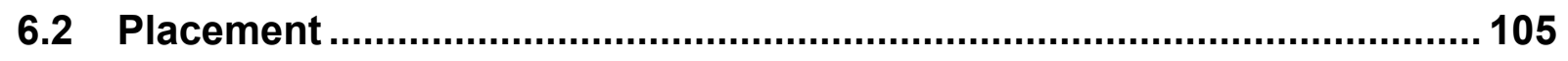

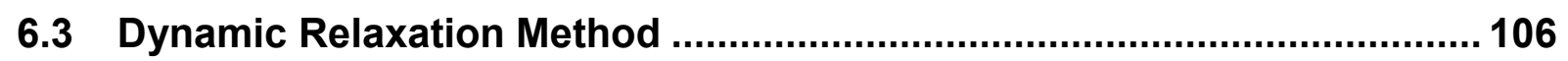

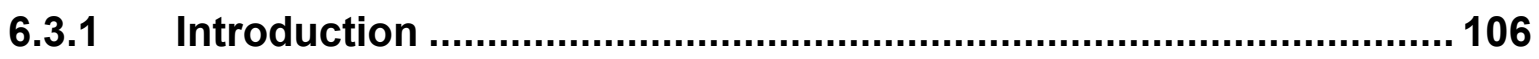

6.3.2 Brief History of Dynamic Relaxation ........................................... 107

6.3.3 Formulation............................................................................... 108

6.3.4 Implementation .......................................................................... 110

6.4 Evaluation of Relaxation on Unconstrained Folded Plug........................ 111

6.5 Relaxation Process on Folded Plug in Storage Position .........................112

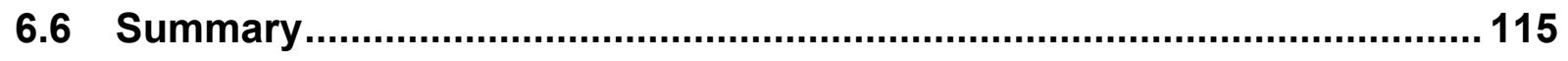


$7 \quad$ Full-Scale Prototype: Deployment ….......................................................... 117

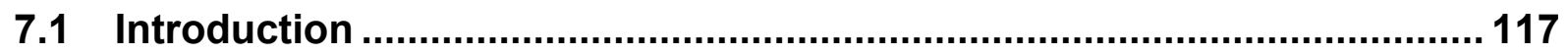

7.2 Initial Configuration Before Deployment................................................117

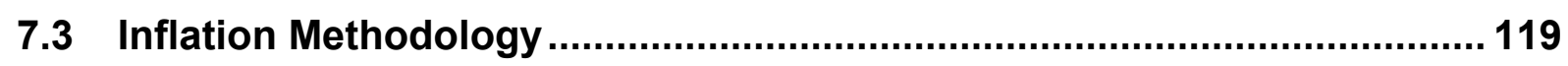

7.3.1 Introduction to Inflation Algorithms ..........................................119

7.3.2 Assumptions for the Deployment Simulation ............................... 121

7.3.3 Uniform Pressure Method .......................................................... 122

7.3.4 Multi-Chamber and Fluid Exchange Setup................................... 126

7.4 Volume Flow Rate Verification .................................................................. 129

7.5 Passive Restraining Mechanism............................................................. 131

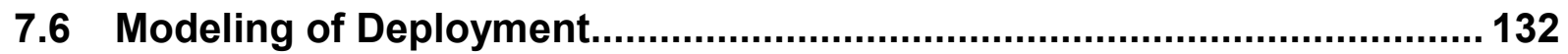

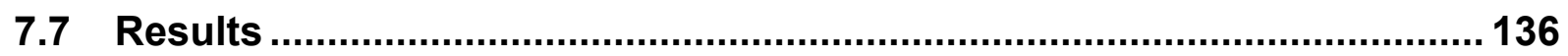

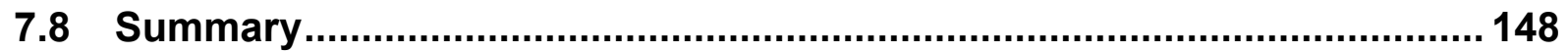

8 Full-Scale Prototype: Evaluation of Axial Slippage ...................................... 150

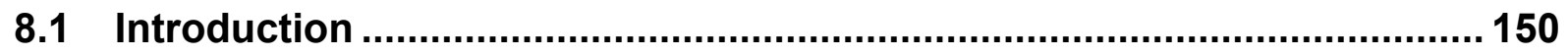

8.2 Induced Axial Slippage: Model Setup ...................................................... 150

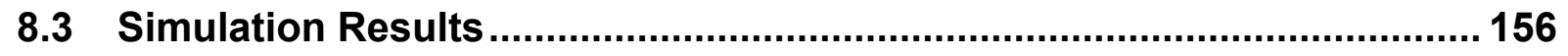

8.3.1 Results for Normal Operating Conditions .................................... 156

8.3.2 Results for Assuming Depressurization of the Plug ..................... 160

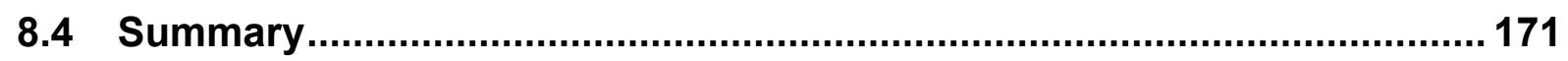

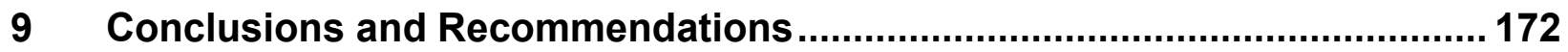

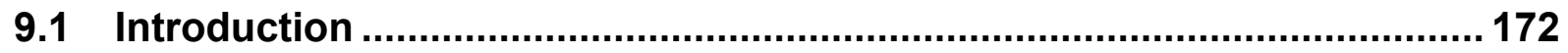

9.2 Conclusions for Reduced-Scale Prototype.............................................. 172

9.2.1 FE Model Creation........................................................................ 173

9.2.2 Mesh Convergence Study …................................................... 173 
9.2.3 Simplified Deployment............................................................... 173

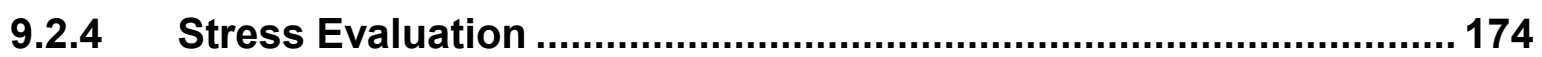

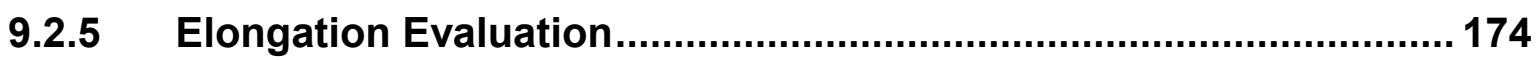

9.2.6 Slippage Evaluation .................................................................. 174

9.3 Conclusions for Full Scale Prototype .................................................. 175

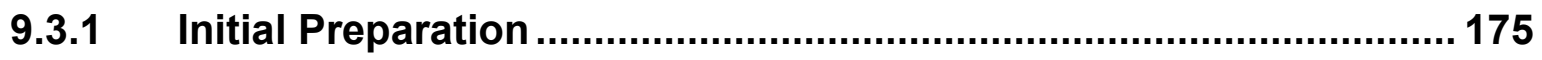

9.3.2 Folding Configuration ................................................................ 176

9.3.3 Placement and Relaxation ........................................................ 176

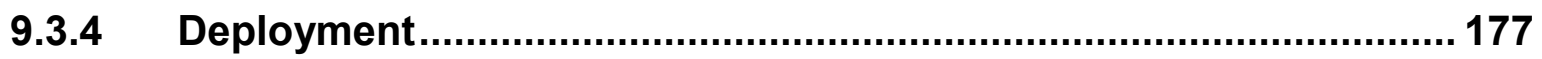

9.3.5 Evaluation of Axial Slippage ..................................................... 178

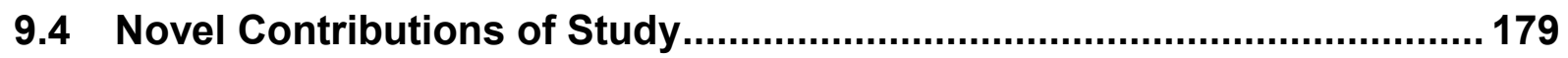

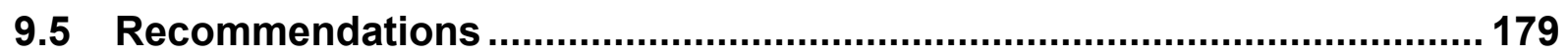

9.5.1 Recommendations for Simulations of Reduced-scale Prototype .. 179

9.5.2 Recommendations for Simulations of Full-Scale Prototype .......... 180

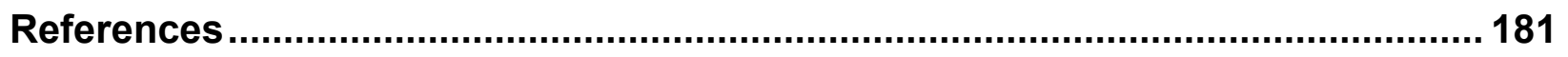

Appendix A: Theoretical Calculation of Stress for Quarter Scale Prototype ....... 194

Appendix B: Theoretical Calculation of Stress for Full Scale Prototype ............. 195

Appendix C: Calculation of Equivalent Cross Sectional Area of Rope ................. 196

Appendix D: Calculation of Elastic Force for a Connector Element...................... 198

Appendix E: Calculation of Equivalent Density for Full-Scale Slippage Simulation. 


\section{Table of Figures}

Figure 1.1 Francesco Lana de Terzi's conceptual vacuum airship (left) and hot air balloon from Montgolfier brothers (right) [3] .................................................... 1

Figure 1.2 Deployment sequence of Bird's air-supported radome [7]..................... 2

Figure 1.3: Inflation sequence of the satellite-balloon Echo 1 [8]........................... 3

Figure 1.4: Aeronautic application: Mars Pathfinder Airbags [10]........................ 4

Figure 1.5: Aeronautic application: Inflatable Lunar Habitats [11]. ......................... 4

Figure 1.6: Automotive application: Automotive Airbag [12] .............................. 5

Figure 1.7: Civil engineering application: Inflatable Composite Bridge [13]......... 5

Figure 1.8: Architectural application: Inflatable Exhibition Dome [7]..................... 5

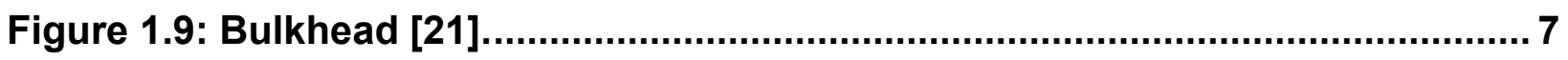

Figure 1.10: Floodgate for city tunnel (left) [22]; underground London (right) [23]. 8

Figure 1.11: Giant grout bag (left) and Inflatable plug (right) [24-25]..................... 9

Figure 1.12: Inflatable Tunnel Plugs from Lindstrand Technologies Ltd, UK [26-27].

Figure 1.13: Schematic of RTP deployment [14-15] ........................................... 10

Figure 1.14: Plugs tested in an actual tunnel in 2008 (left) [14-15, 28], and in testing facilities at WVU in 2012 (right) [29]................................................... 12

Figure 2.1: Conceptual Schematic of the finite element procedure........................ 15

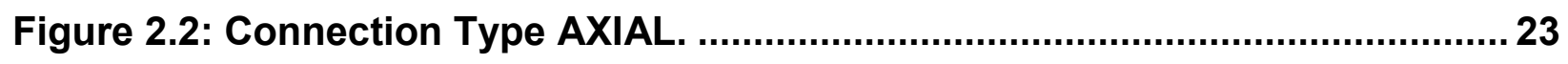

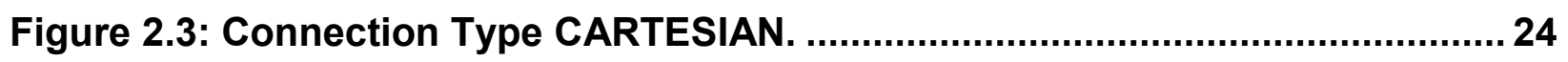

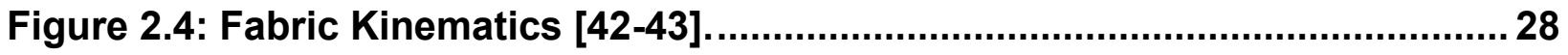

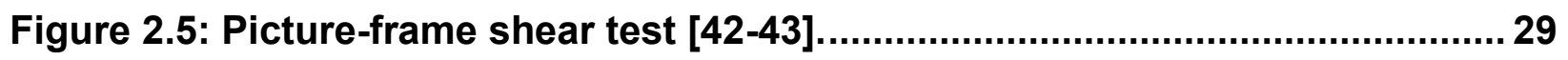

Figure 2.6: Cavity reference node of the fluid cavity [42-43]............................... 34

Figure 2.7: Scenario of tunnel leakage that causes flooding in the tunnel.............38

Figure 2.8: Schematic of hydrostatic pressure distributions................................. 39

Figure 3.1: Detail of layers of fabric used for the construction of the membrane of reduced and full scale prototypes. ...................................................................... 48

Figure 3.2: Dimensions of reduced scale prototype. ............................................52 
Figure 3.3: Convergence plot for FE Model of unconstrained reduced-scale

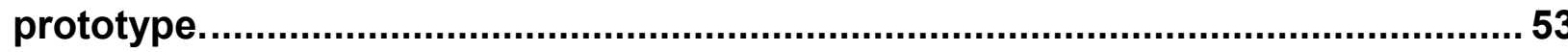

Figure 3.4: Sequence of simplified deployment of reduced-scale prototype. ....... 55

Figure 3.5: Reduced-scale FE model under unconstrained pressurization........... 57

Figure 3.6: Schematic of Constrained Elongation Measurement [59] .................... 58

Figure 3.7: Pressurization Procedure for Elongation Test of Constrained Reduced

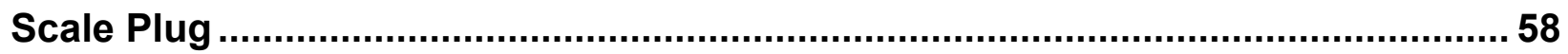

Figure 3.8: Experimental setup for reduced scale prototype tested under

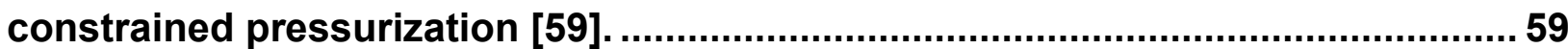

Figure 3.9: Diagram of the constitutive model used in the simulations.................60

Figure 3.10: (a) Schematic of Axial Slippage Model; (b) Longitudinal Cut-view of

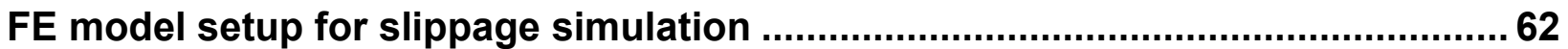

Figure 3.11: (a) Scenario of decreasing inflation pressure $\left(P_{i}\right)$. (b) Scenario of

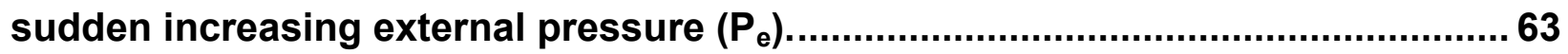

Figure 3.12: Selected Pressurization Sequence for Induced Slippage of

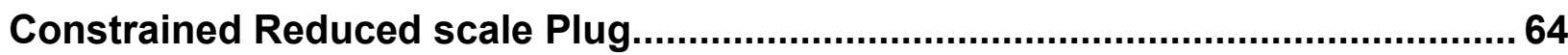

Figure 3.13: Contour of Hoop and longitudinal stresses obtained from FE results

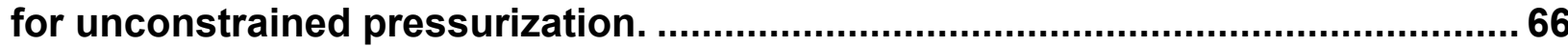

Figure 3.14: FE model performs the elongation test under constrained condition. 67

Figure 3.15: Plot of material properties evaluation. ................................................ 67

Figure 3.16: Error of material candidates respect of experimental results............69

Figure 3.17: Contour of Hoop and longitudinal stresses obtained from FE results

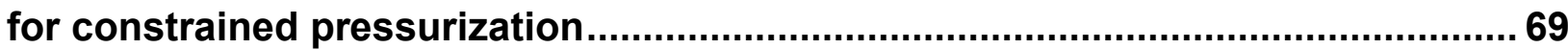

Figure 3.18: Slippage results of same friction coefficient $(0.15)$ with various loading combinations. ........................................................................................ 71 Figure 3.19: Slippage results of same friction coefficient $(0.19)$ with various

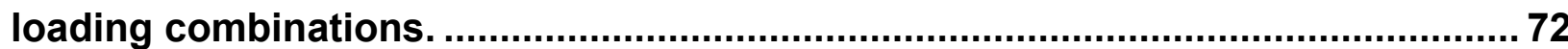

Figure 3.20: Slippage results of same friction coefficient $(0.25)$ with various

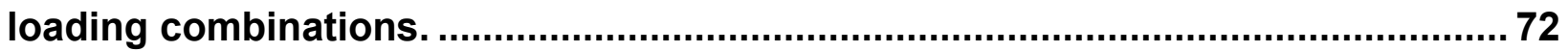


Figure 3.21: Slippage results of same loading combinations (55/40) with various friction coefficients. 73

Figure 3.22: Slippage position for pressure (55/40) with various friction coefficients. 74

Figure 3.23: Slippage results for design pressure (68/48) with various friction coefficients. 75

Figure 4.1: Nominal Dimensions of Full-Scale Prototype....................................... 78

Figure 4.2: Isometric View of Actual Full Scale Prototype. .................................... 78

Figure 4.3: (a) Initial Geometry and (b) Mesh Configuration. .................................. 79

Figure 4.4: Hoop Stress of Model with 30000 elements (20000 DOF).................... 81

Figure 4.5: Longitudinal stress of Model with 30000 elements (20000 DOF)......... 81

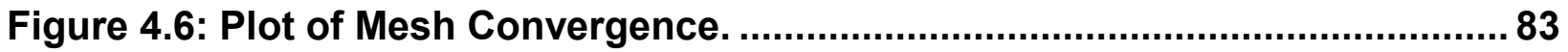

Figure 4.7: Geometry (left) and Mesh Configuration (right) of Tunnel. ..................84 84

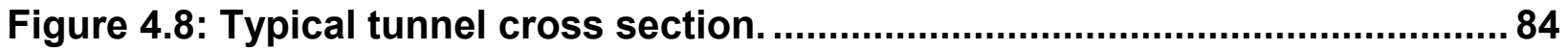

Figure 4.9: Geometry (left) and Mesh Configuration (right) of Base....................... 85

Figure 4.10: Geometry (left) and Mesh Configuration (right) of Rotational Plate. . 85

Figure 4.11: Components of the FE model for full-scale prototype........................ 85

Figure 5.1: (a) Tuck fold, (b) Thin fold [71], (c) Thick fold [72], (d) Simple fold [73],

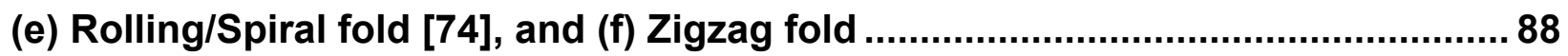

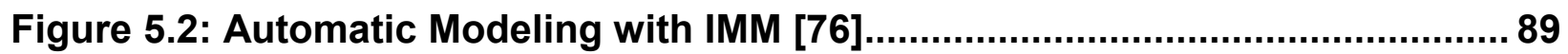

Figure 5.3: Folding sequence of the polyhedron approximation geometry [78].... 89

Figure 5.4: A zigzag fold using rigid plates [79] ................................................ 90

Figure 5.5: Simulation of folding as part of the manufacturing process [73]....... 90

Figure 5.6: Mass scaling factor versus percentage difference between kinetic and internal energy 94

Figure 5.7: (a) Flattened cylindrical region. (b) Schematic of folding sequence

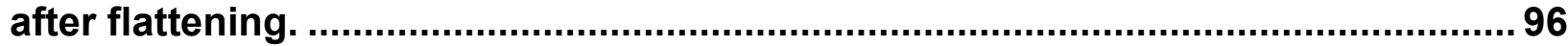

Figure 5.8: View of initial condition from XY-plane (left) and isometric (right)..... 97

Figure 5.9: Translation of Line $A$ and Line $B$ along the $X$-axis............................... 97

Figure 5.10: Gravity force acting on the deflated plug along the Y-axis................ 98

5.11: Line $C$ translates along the $Y$-axis.................................................................... 99 


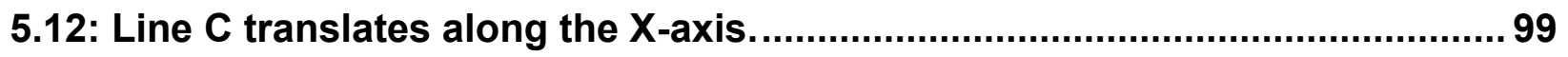

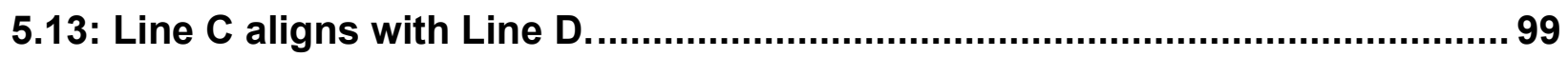

Figure 5.14: Initial condition for Folding Part II. ................................................ 100

Figure 5.15: Rotational Plate \#1 translates along the $\mathrm{Y}$-axis................................ 100

Figure 5.16: (a) Rotational Plate \#1 rotates clockwise $120^{\circ}$ about the Z-axis. (b)

Gravity force acting to complete the fold............................................................. 101

Figure 5.17: Rotational Plate \#2 translates along the Y-axis............................... 101

Figure 5.18: (a) Rotational Plate \#2 rotates clockwise $135^{\circ}$ about the Z-axis. (b)

Completion of second folding by action of gravity force. ................................. 101

Figure 5.19: Rotational Plate \#3 translates along the Y-axis................................. 102

Figure 5.20: (a) Rotational Plate \#3 rotates clockwise $135^{\circ}$ about the Z-axis. (b)

Completion of third folding by action of gravity force........................................... 102

Figure 5.21: Line $B$ of plug translates along the $Y$-axis...................................... 103

Figure 6.1: Location of Storage Area inside a Tunnel.......................................... 104

Figure 6.2: (a) Initial condition for placement; (b) Orientation of folded plug after

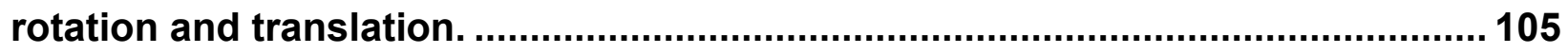

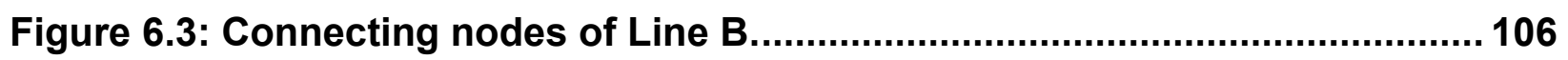

Figure 6.4: Connecting nodes after placement simulation................................... 106

Figure 6.5: Kinetic Energy Plot Pattern of Dynamic Relaxation [90-91]. .............. 109

Figure 6.6: Evaluation of influence of relaxation through volume comparison... 111

Figure 6.7: Model with Dynamic Relaxation @ t=25 sec in Figure 6.6. Deformation

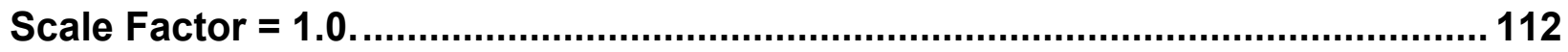

Figure 6.8: Model without Dynamic Relaxation @ $\mathrm{t}=25 \mathrm{sec}$ in Figure 6.6.

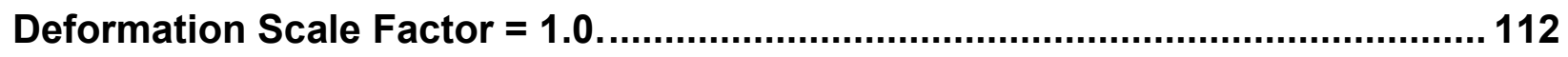

Figure 6.9: Rigid plane before closing the storage area..................................... 113

Figure 6.10: Rigid plane after closing the storage area. .....................................113

Figure 6.11: Folded plug after implementation of dynamic relaxation. ................ 113

Figure 6.12: Restitution of Total Area in the Folded plug after relaxation. ........... 114

Figure 6.13: Evaluation of Energies during the Implementation of Dynamic

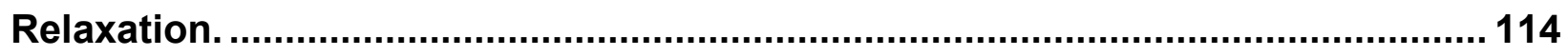


Figure 6.14: Evaluation of Kinetic Energies during the Implementation of Dynamic Relaxation. 115

Figure 7.1: Initial position of the folded plug before deployment. 118

Figure 7.2: (a) Eulerian Lagrangian Interaction Algorithm [119] and (b) Meshfree Algorithm [120]. 120

Figure 7.3: Definition of multi-chamber in an inflatable tube [145]...................... 126

Figure 7.4: Definition of multi-chamber in an inflatable modeled by Slade et al. [146] 126

Figure 7.5: Flow rate verification. 131

Figure 7.6: Actual deployment of a full-scale prototype performed at the WVU testing facility [58]. 133

Figure 7.7: Position of chamber walls. 134

Figure 7.8: Undirected air flow schema (Deployments \#1, \#2, and \#3)................ 135

Figure 7.9: Directed air flow schema (Deployment \#4). 136

Figure 7.10: Actual deployment. (a) Front view with details of local conformity, and (b) Wrinkle view from the interior of the plug. 137

Figure 7.11: Deployment \#1. (a) Front view and (b) Wrinkle view. (Deformation Scale Factor $=1$ ) 139

Figure 7.12: Deployment \#2. (a) Front view and (b) Wrinkle view. (Deformation Scale Factor $=1$ ) 140

Figure 7.13: Deployment \#3. (a) Front view and (b) Wrinkle view. (Deformation Scale Factor $=1$ ). 141

Figure 7.14: Deployment \#4. (a) Front view and (b) Wrinkle view. (Deformation Scale Factor $=1)^{2}$ 142

Figure 7.15: Energy Trace of Deployment \#4 ....................................................... 144

Figure 7.16: Area Restoration in Deployment \#4................................................. 145

Figure 7.17: Deployment behavior of Deployment \#1 and \#2................................ 146

Figure 7.18: Deployment behavior of Deployment \#3 and \#4............................... 147

Figure 8.1: Pressurization event for normal operating conditions ....................... 152

Figure 8.2: Pressurization Scheme for Internal and External Pressures. ............. 152 
Figure 8.3: Sequence of pressurization and depressurization to induce slippage

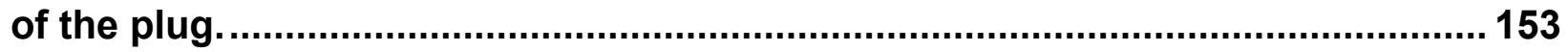

Figure 8.4: Cut view of FE model setup for slippage scenarios \#1 and \#2. ......... 155

Figure 8.5: Cut view of Loading Scenario \#1 with hidden solid elements of fluid

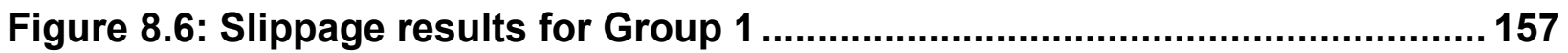

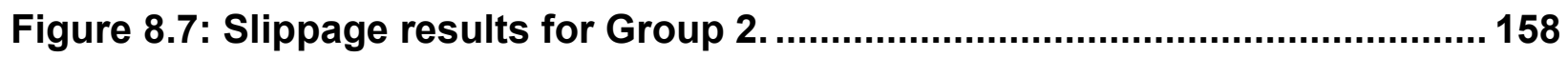

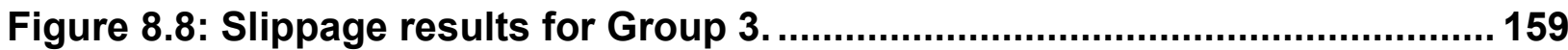

Figure 8.9: Slippage results for Group 4.......................................................... 159

Figure 8.10: Slippage results for Group 5........................................................ 161

Figure 8.11: Sequence of slippage for friction coefficients of 0.13 and 0.15

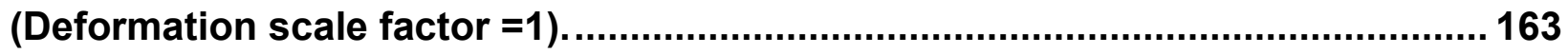

Figure 8.12: Sequence of slippage for friction coefficients of 0.19 and 0.25 .

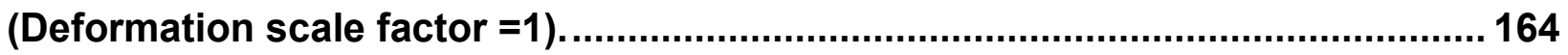

Figure 8.13: Slippage results for Group 6.......................................................... 166

Figure 8.14: Sequence of slippage for friction coefficients of 0.13 and 0.15

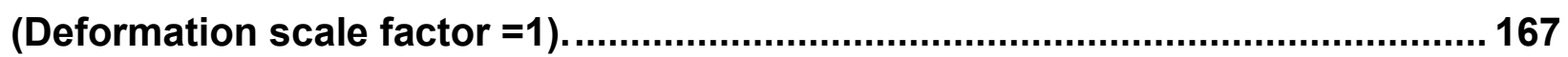

Figure 8.15: Sequence of slippage for friction coefficients of 0.19 and 0.25 .

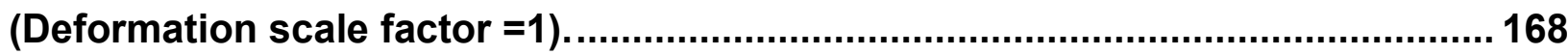

Figure 8.16: Slippage result for Group 7........................................................ 169

Figure 8.17: Slippage result for Group 8. ....................................................... 170 


\section{Table of Tables}

Table 3.1: Material properties of the structural membrane ...................................48

Table 3.2: Hoop stress results for unconstrained reduced- scale prototype. .......54

Table 3.3: Candidate of material properties...........................................................60

Table 3.4: Load combinations of maximum internal and external pressure..........64 64

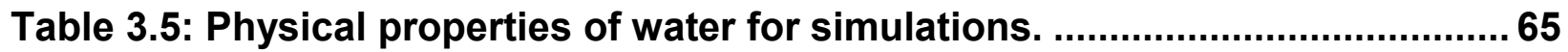

Table 3.6: Stress evaluation of reduced scale prototype for unconstrained

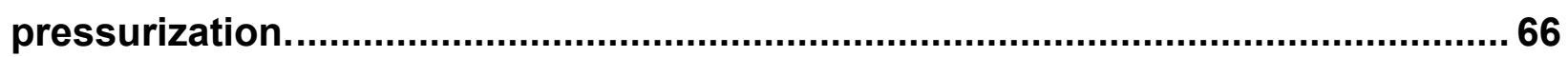

Table 3.7: Summary of holding resistance ratios for different friction coefficients.

Table 4.1: Stress evaluation results of unconstrained full-scale prototype. ..........80 80

Table 4.2: Summary of convergence study using hoop stress as control

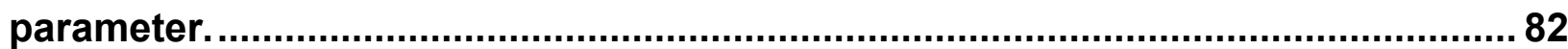

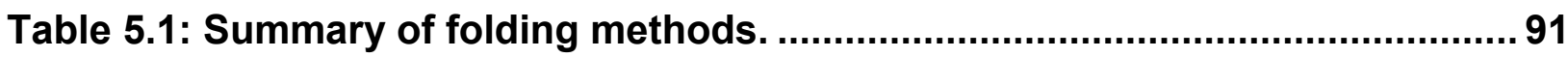

Table 5.2: Computational cost corresponding to mass scaling factor....................94

Table 7.1: Material Properties of Equivalent Membrane ......................................119

Table 7.2: Physical Properties of Air [136-139] ............................................. 125

Table 7.3: Test Plan of Deployment Simulations.................................................... 134

Table 7.4: Contact area after completion of deployment....................................... 143

Table 8.1: Physical properties of water and air. .................................................... 151

Table 8.2: Loading Scenarios for Induced Slippage. ............................................. 154

Table 8.3: Complete Induced Slippage FE Simulation Plan ................................. 156

Table 8.4: Influence of Friction Coefficient on Holding Resistance Ratio............. 161

Table 8.5: Influence of Friction Coefficient on Holding Resistance Ratio............. 166

Table 8.6: Summary results of holding resistance of Groups 5 to 8 . ..................171 


\section{Introduction}

\subsection{Origin, Evolution, and Applications of Inflatable Structures}

The history of inflatable structures was firstly begun centuries ago in the field of aeronautical engineering. In 1670, Francesco Lana de Terzi, an italian mathematician, proposed the concept of using Archimedes' buoyancy principle to vacuum the spheres shown in Figure 1.1. These spheres were made of very thin copper foil, so that they would be lighter than the air and lift a boat into the air [1]. Although Lana de Terzi's airship with vacuum spheres had never been built, the idea still influences scientists in their studies on the field of flight. Joseph and Etienne Montgolfier constructed a balloon with linen, covered by stiff paper and anchored the balloon with ropes in 1780 (Figure 1.1). The Montgolfier brothers successfully launched the first manned balloon by delivering heated air (which is lighter than the air surrounding outside the balloon) into the balloon [2].
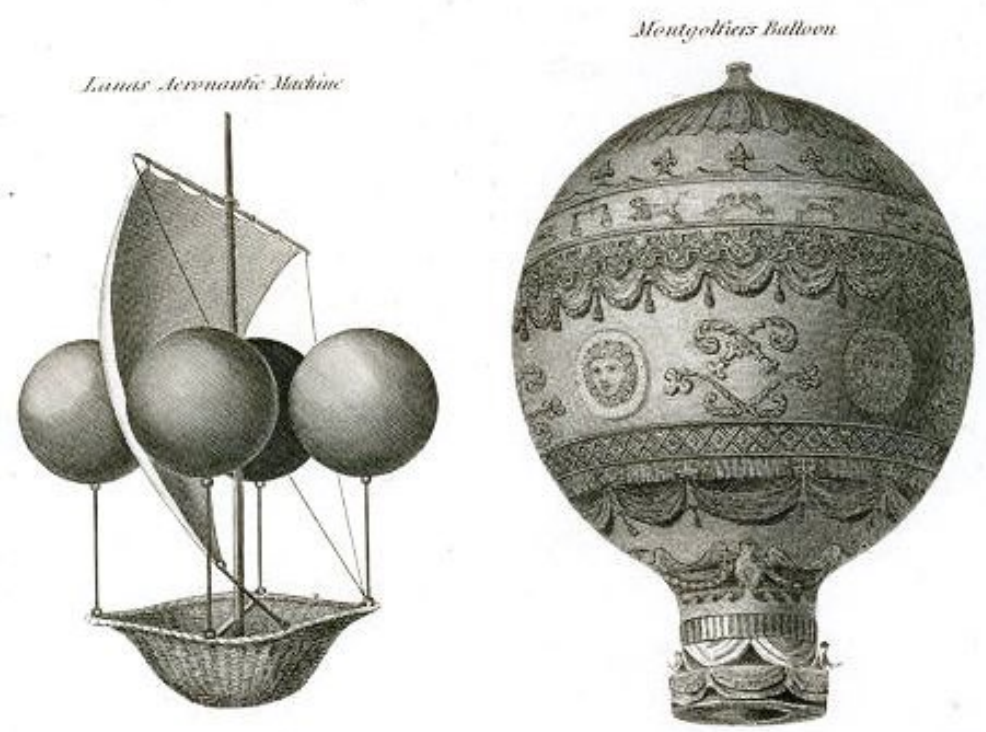

Figure 1.1 Francesco Lana de Terzi's conceptual vacuum airship (left) and hot air balloon from Montgolfier brothers (right) [3]. 
Other application of inflatable structures was the one developed by R. W. Thomson who invented the pneumatic tire using india-rubber for which he was granted a patent in the US in 1847 [4]. Thomson's pneumatic tire consists of a hollow tube, screw caps, and enclosed cover. The hollow tube was inflated with air, sealed with screw caps, riveted on the wheel, and covered by a leather cover for protection purposes [4]. When people commenced to use rubber and nylon widely in 1940s, the inflatable structures started to be used in military applications as vehicle tires and decoys.

After World War II ended, Walter Bird received research funding from U.S. military to design a shelter to enclose and protect the large surveillance radar antennas from extreme weather conditions [5]. To avoid interference with the radar signals, the shelters had to be non-metallic. Walter Bird developed an air-supported radome that was made of thin neoprene-coated fiberglass fabric in 1946 [6]. The invention of fabric membranes would later play an important role on the development of inflatable structures. Figure 1.2 shows Bird's concept, in which a metal frame was initially used to deploy the thin fabric into a dome-shape and later inflated with air.
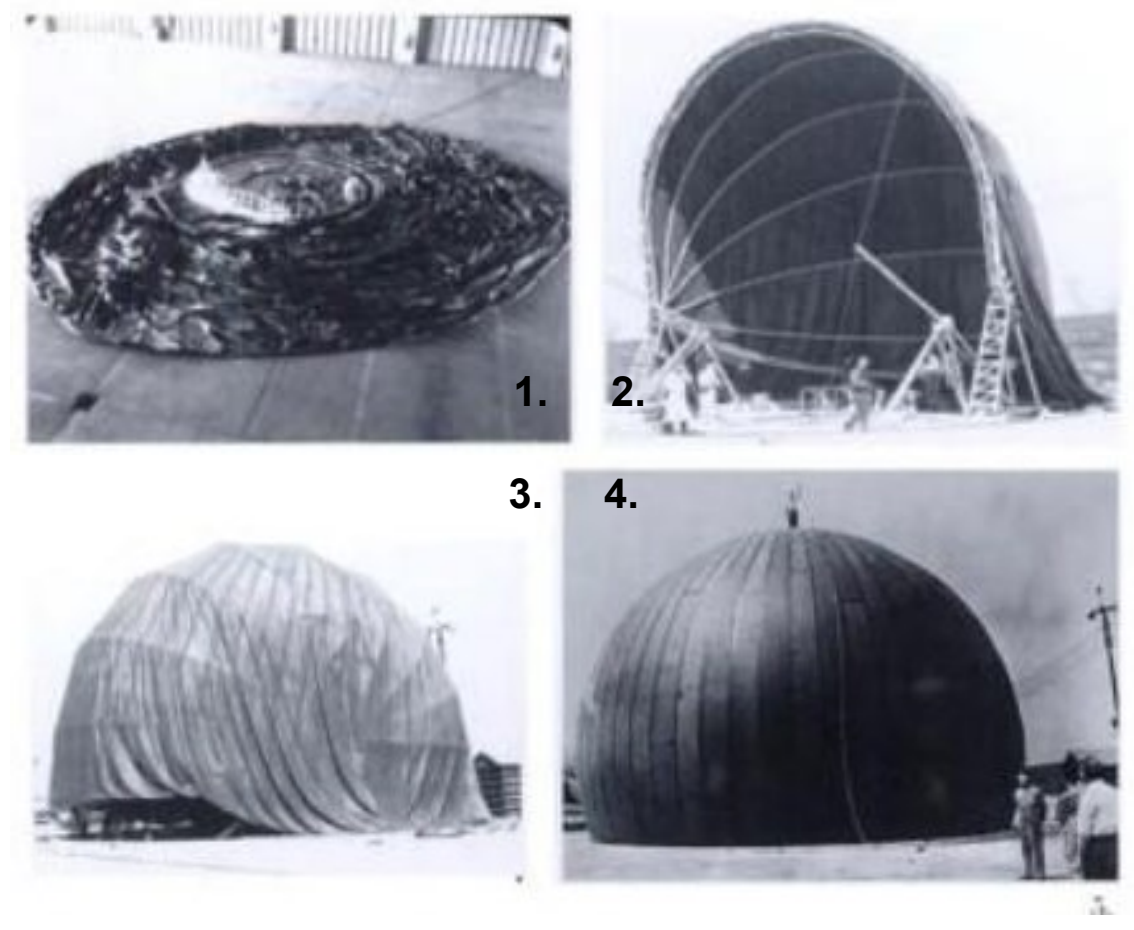

Figure 1.2 Deployment sequence of Bird's air-supported radome [7]. 
After several launch attempts, Echo 1, the passive satellite-balloon was launched successfully and inflated perfectly by the National Aeronautics and Space Administration (NASA) in 1960. The 30.5 meter of diameter "satelloon" was made of an aluminized, Mylar polyester film with a thickness only $12.7 \mu \mathrm{m}$. Echo 1 wrote an evolutionally new chapter on space technology. Figure 1.3 shows the inflation testing of Echo 1 which took place in a navy hangar at Weeksville, NC before the actual launch [8].

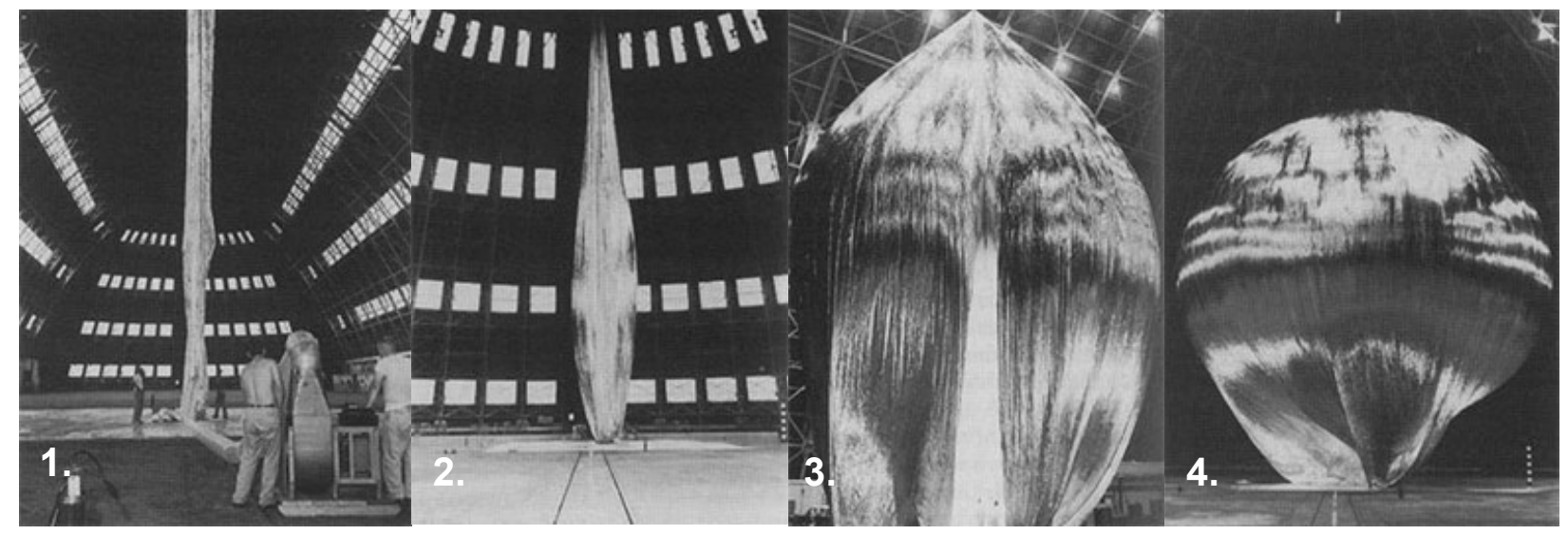

Figure 1.3: Inflation sequence of the satellite-balloon Echo 1 [8].

Walter Bird's pioneer contribution to the developing the commercial applications of the pneumatics and NASA's Echo 1 ignited the era of academic research on inflatable structures. Frei Otto [9] implemented advanced mathematics for the design of the structural form of pneumatic constructions. Since then, inflatable structures have been innovated and developed widely in a variety of designs, shapes, and sizes for aeronautic, automotive, civil engineering, and architectural applications. For example, some of the aeronautic applications included the landing airbag for the Mars Pathfinder project [10] and the inflatable lunar habitat developed in the NASA Langley Research Center [11] shown in Figure 1.4 and Figure 1.5, respectively. The automotive industry developed the safety airbag in vehicles [12] that have been widely implemented to protect the passengers during car accident (Figure 1.6). Other application is illustrated in Figure 1.7, where a conventional bridge was built by the combination of inflatable composite arch structures and concrete [13]. The inflatable structural fiber tubes were inflated and infused with resin used to form lightweight hollow arches at the work site. 
The hollow arches then occupied with concrete and covered by the composite panels to support the roadway. For architectural applications, inflatable domes such as the one illustrated in Figure 1.8 are common structures used in exhibition events to provide shelter from weather with significant outward appearance and shape. Most recently, a new application for high pressure confined inflatable structures was developed for sealing tunnels segments to prevent spread of smoke, fumes, and flooding. The feasibility of implementation of this concept is presentenced in Ref. [14] and [15].

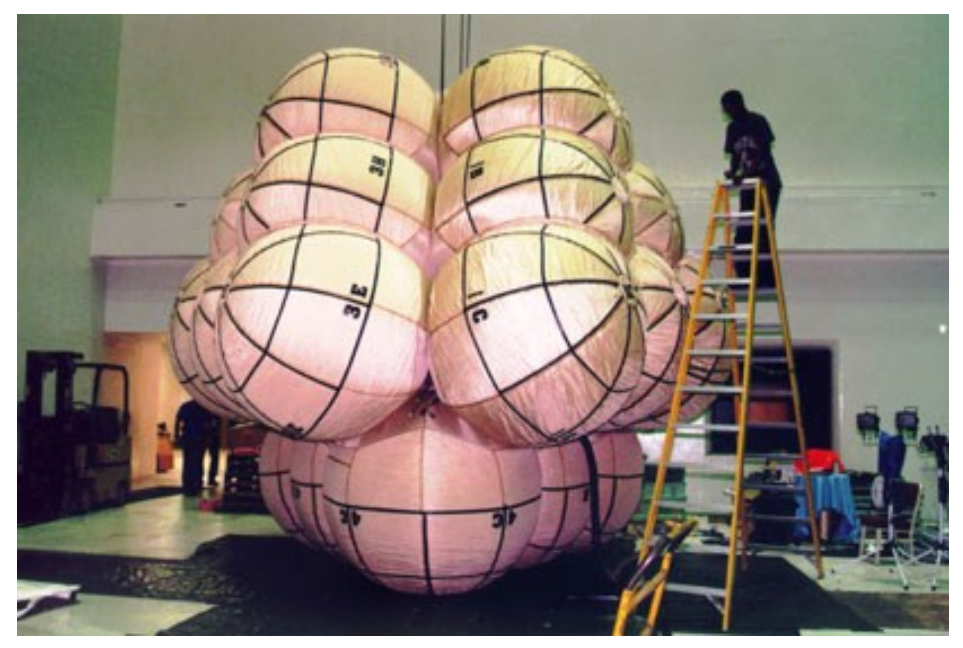

Figure 1.4: Aeronautic application: Mars Pathfinder Airbags [10].

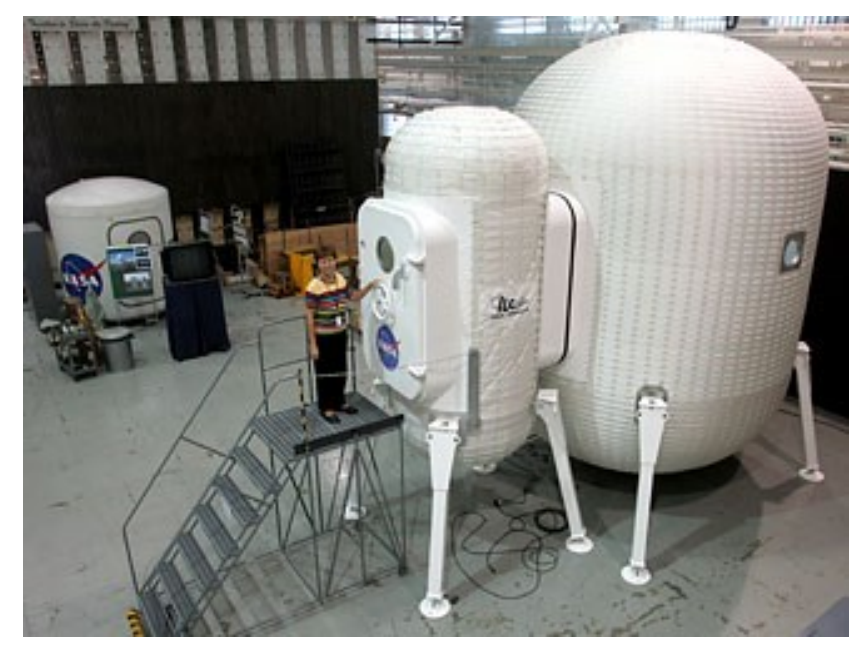

Figure 1.5: Aeronautic application: Inflatable Lunar Habitats [11]. 


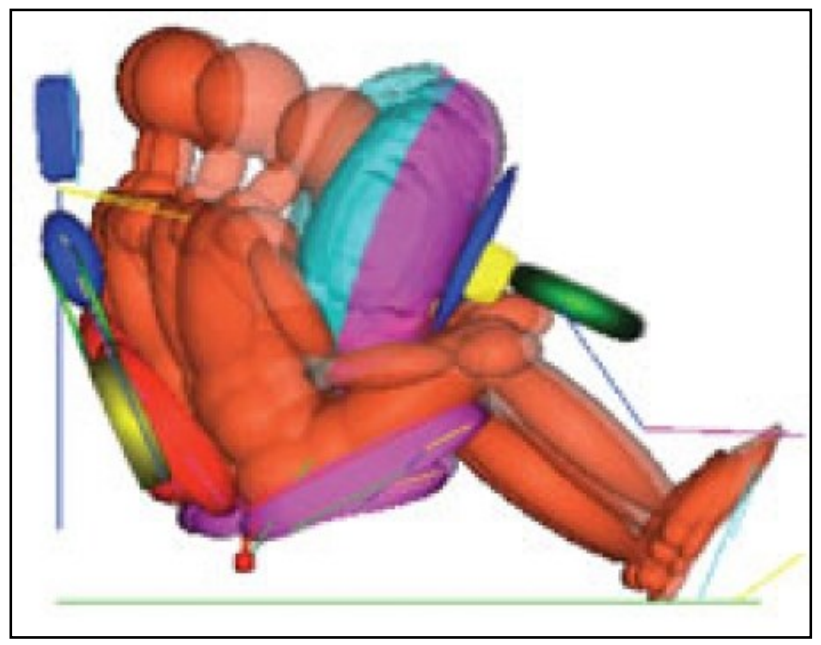

Figure 1.6: Automotive application: Automotive Airbag [12].

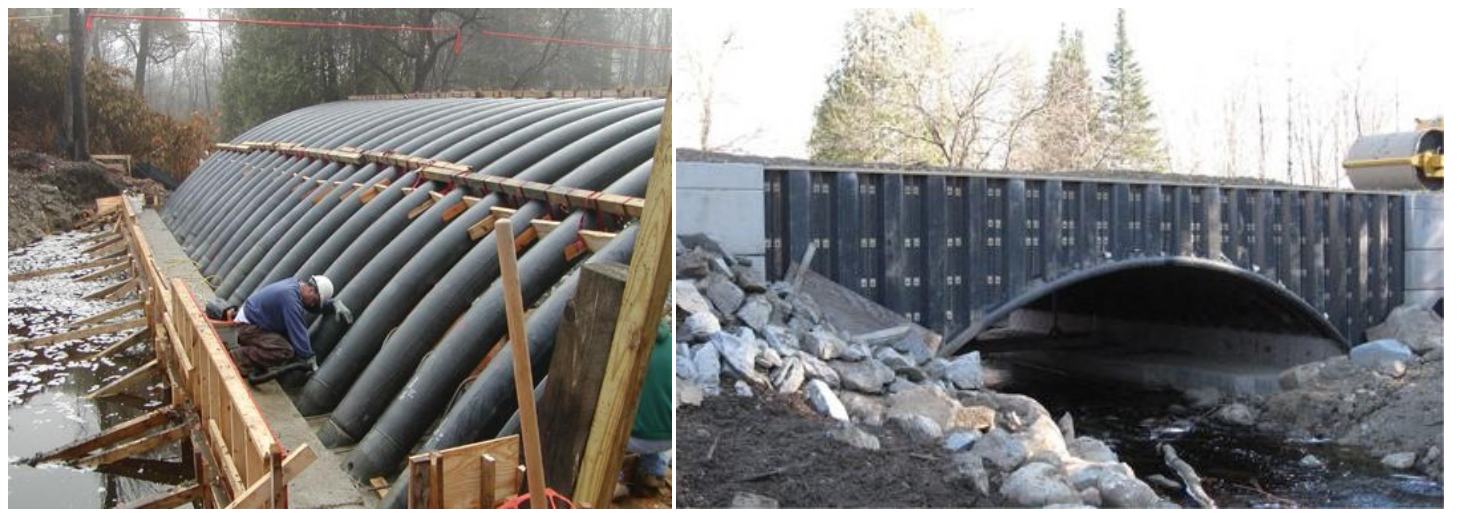

Figure 1.7: Civil engineering application: Inflatable Composite Bridge [13].

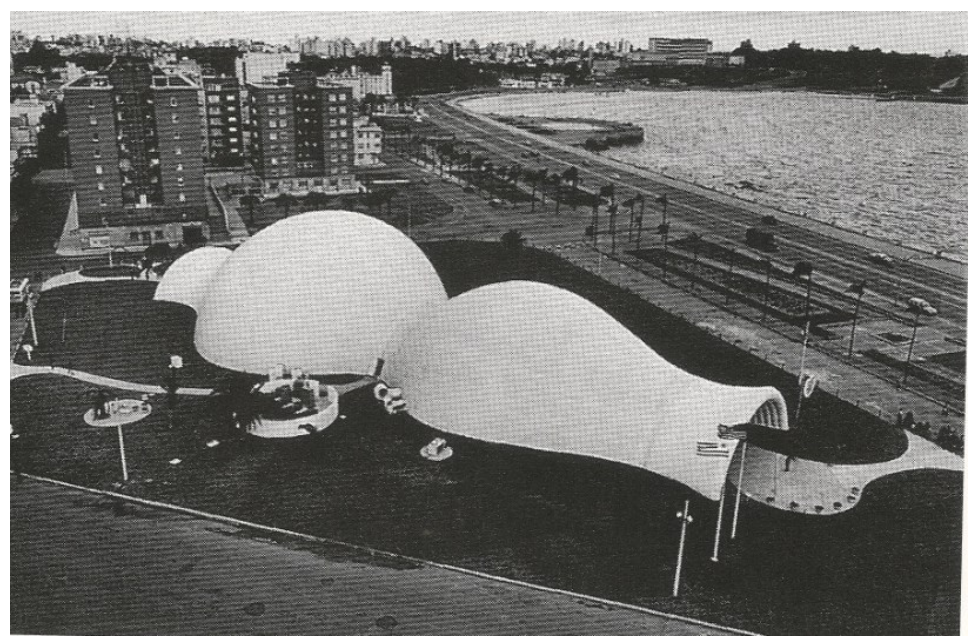

Figure 1.8: Architectural application: Inflatable Exhibition Dome [7]. 


\subsection{Problem Statement}

In the last few decades, the protection of critical civil infrastructure has become a difficult task for different government agencies around the world. Specifically, tunnels have been identified as particularly vulnerable to different threats that require additional or alternative protection systems as well as mitigation technologies. Typically, a tunnel is an underground passage which is enclosed but open for ingress and egress at the ends. Most of the tunnels are constructed completely under the ground, but some are built across the bottoms of bodies of water, such as rivers, bays or lakes. Tunnels can be categorized into three main types:

I. Mine Tunnels (used for delivering miners, equipments, and mineral back and forth from deep earth during ore extraction)

II. Public Works Tunnels (used for carrying water, sewage, and gas lines)

III. Transportation Tunnels (used for traveling and shipping such as railway tunnels, roadway tunnels, and pedestrian tunnels)

Safety of transportation tunnels (III) is a very important aspect in the operation of a tunnel system and it's always a challenge to create or integrate a protection system to prevent or mitigate the occurrence of hazardous events. The most common hazardous scenarios include:

a. Fire/smoke: Any active or post conflagration condition causes heat, smoke and harmful vapors. An example of this scenario is the Jungangno Subway Station Arson Fire which happened in Korea in 2003 [16].

b. Flooding: The condition of overflowing of water onto tunnel area as some examples in the US include the Chicago Freight Tunnel Flood in 1992, and the MTA tunnel flood in New York during Hurricane Sandy in 2012 [17, 20].

c. Introduction of Hazardous Materials: Any discharge of fatal chemical, biological, or radiological agents into the tunnel possibly in a form of liquid, solid, or gas like in the Subway Sarin Gas Attack which happened in Japan in 1995 [18]. 
d. Explosion: A violent gas release during the decomposition of explosive substances like in the Moscow Subway Suicide Bombing which happened in Russia in 2004 [19].

e. Structural Integrity Loss: Any reduction of the fitness of the tunnel to carry passengers/freight by human factors or natural causes.

The countermeasures for preventing or mitigating the occurrence of hazardous events can be classified into permanent solutions and temporary solutions. The permanent solutions usually have relatively high sealing efficiency due to their solid and rigid sealing mechanisms such as metallic bulkheads used in New Zealand [21] (Figure 1.9), floodgates for city tunnels in Malaysia [22] (Figure 1.10), and underground London [23] (Figure 1.10). However, they can be very expensive and sometimes difficult to build, install, or maintain over long periods of time.

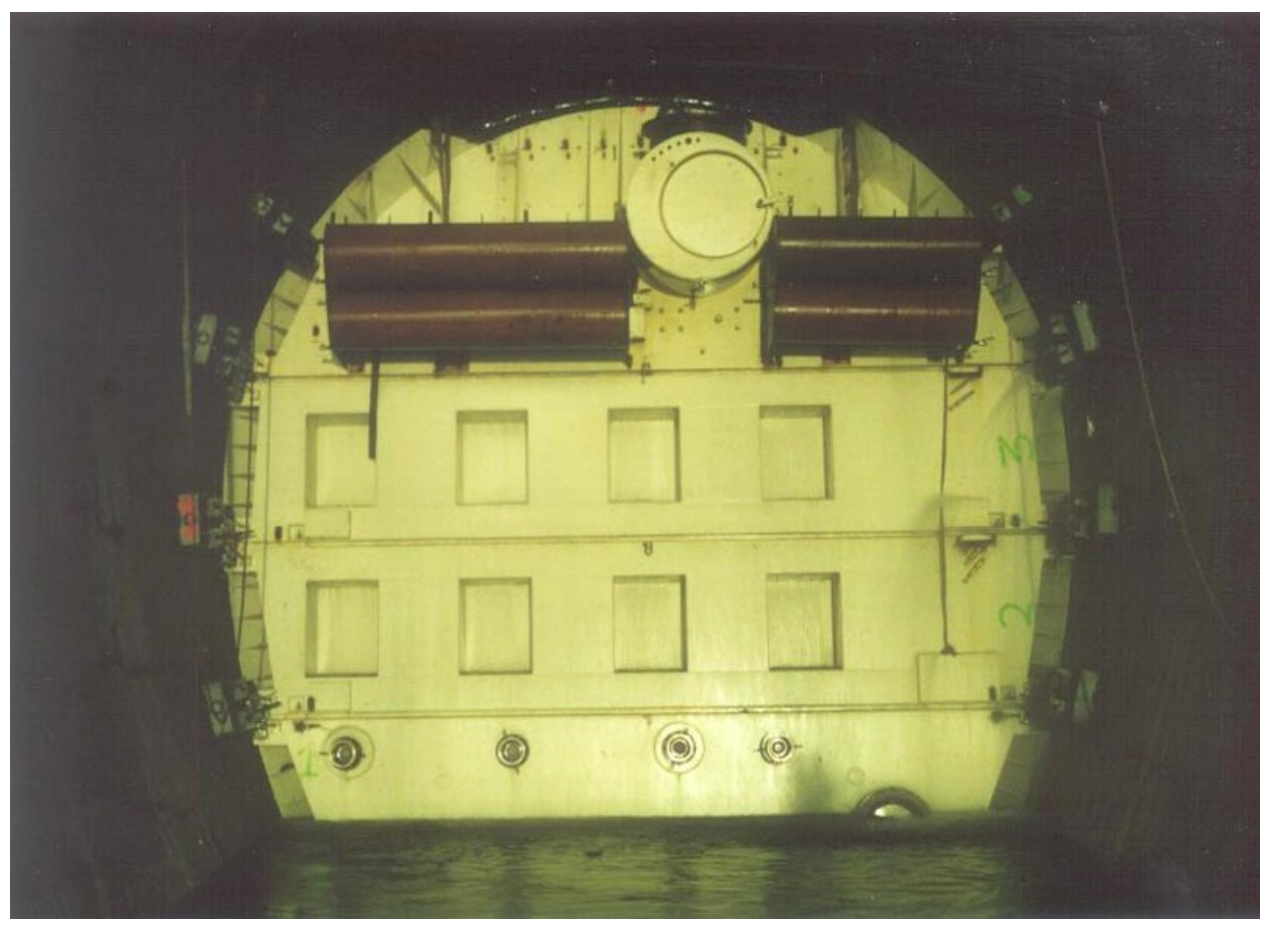

Figure 1.9: Bulkhead [21]. 

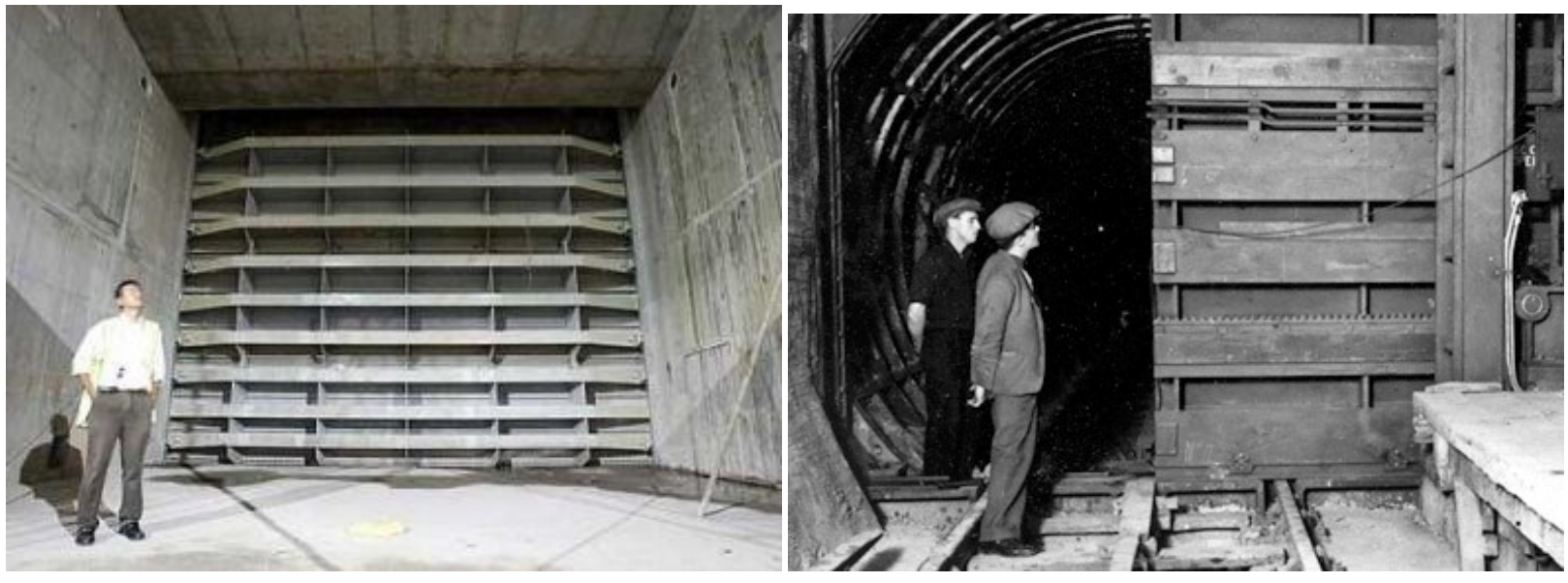

Figure 1.10: Floodgate for city tunnel (left) [22]; underground London (right) [23].

On the other hand, the temporary solutions can be relatively low cost and easy to install while permanent solutions or repairs are implemented. Temporary solutions such as inflatable plugs served in various tunnel events. There are several examples of application of temporary solutions for sealing tunnels. Among them we can cite the case of Petersen Products Co. who manufactured large grout bags and inflatable plugs (Figure 1.11) for emergency, abandonment or maintenance purposes by sealing off mine shafts, canals, or tunnels sections [24-25]. In Europe, Lindstrand Technologies Ltd. [26] from United Kingdom manufactures the inflatable plugs (Figure 1.12) using glass fiber fabric coated with solvent free silicone rubber and aluminum flakes that are able to resist temperatures up to $450^{\circ} \mathrm{C}$. The fabric is burned into sand without releasing any toxic gases if the temperature exceeds the limit of the fabric. The full-scale seals have been tested and proved the ability of extinguishing fire in the tunnel of the Brenner Pass in Italy and in the Hubertustunnel in Holland [27]. The solution proposed by Lindstrand Technologies Ltd. is also installed in the London Underground Victoria Line. 


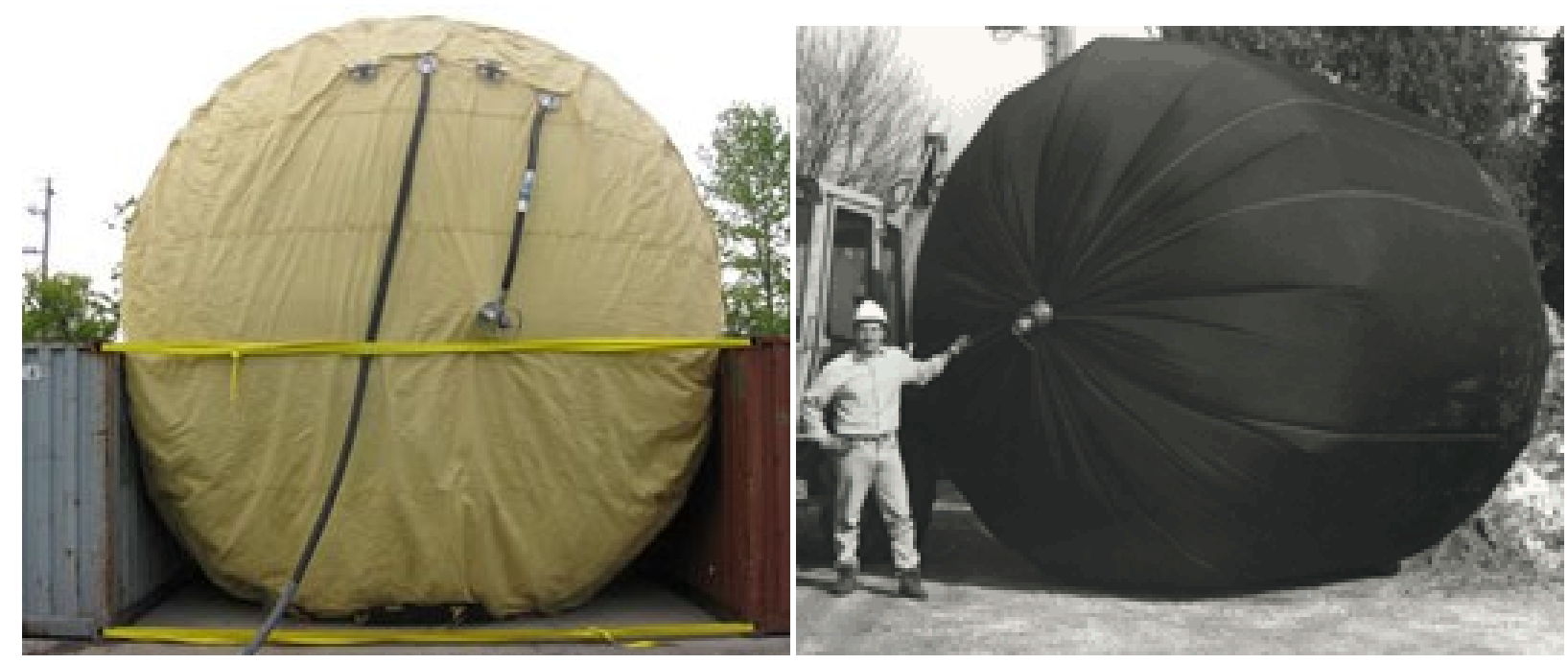

Figure 1.11: Giant grout bag (left) and Inflatable plug (right) [24-25].
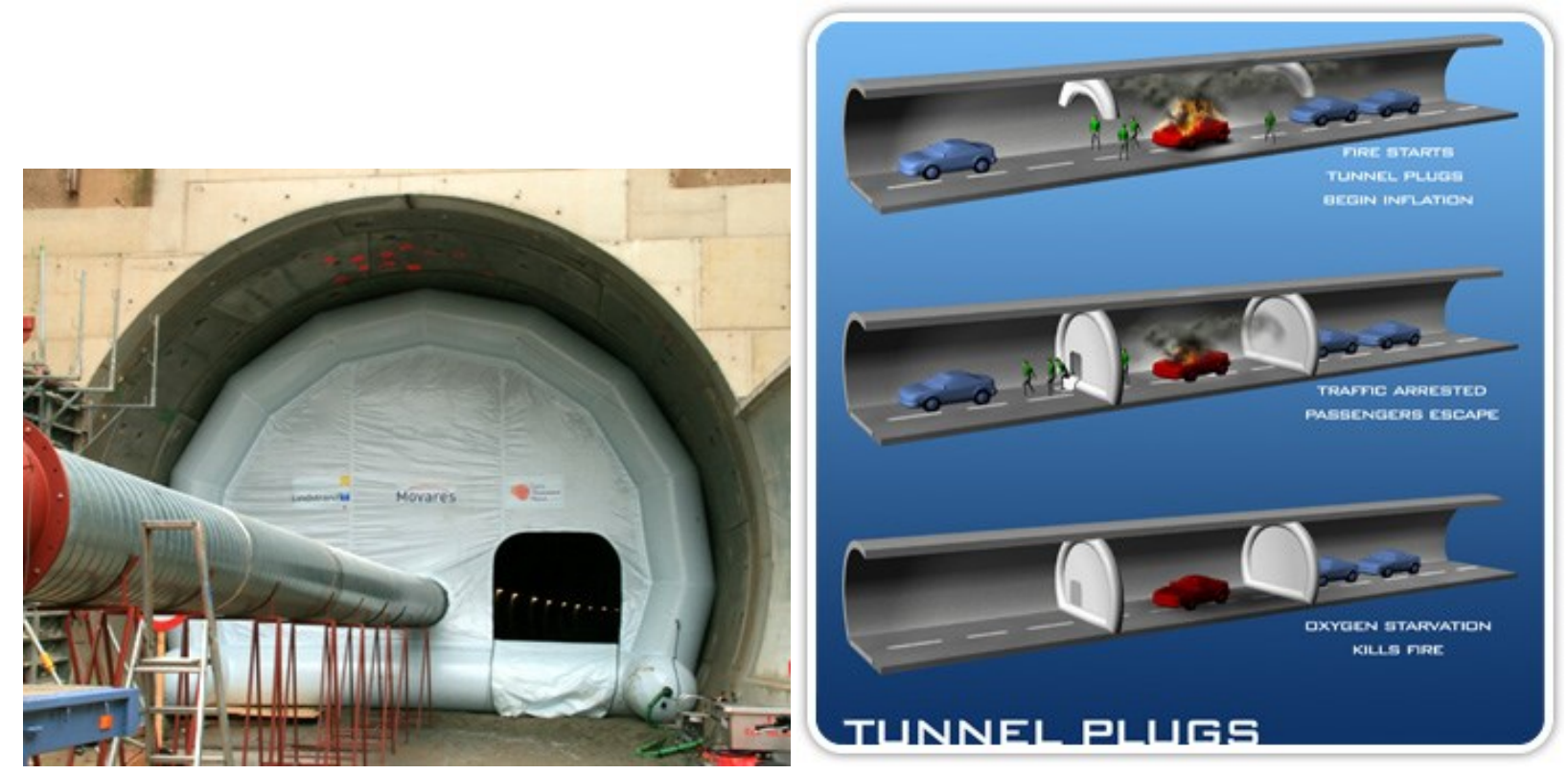

Figure 1.12: Inflatable Tunnel Plugs from Lindstrand Technologies Ltd, UK [26-27].

\subsection{Resilient Tunnel Plug (RTP) System}

The Resilient Tunnel Plug (RTP) System is a passive tunnel protection system developed at West Virginia University (WVU) which was proposed in 2007 [14-15] as a way to prevent or minimize damage induced by hazardous events by creating a compartment inside the tunnel to contain the threat. The RTP system consists in the 
installation of inflatable structures (or inflatable plugs) at specific locations of the tunnel to seal off the tunnel and create a compartment to isolate the compromised region. Figure 1.13 shows conceptually the RTP deployment [14-15]. The inflatable plugs are folded and placed into a container which is later transported to a specific section and pre-installed within the tunnel. When the RTP is activated, the plugs are released from the container, then inflated and pressurized in order to maximize the sealing effect and eventually stop the threat which can be flooding, gases, or fumes.

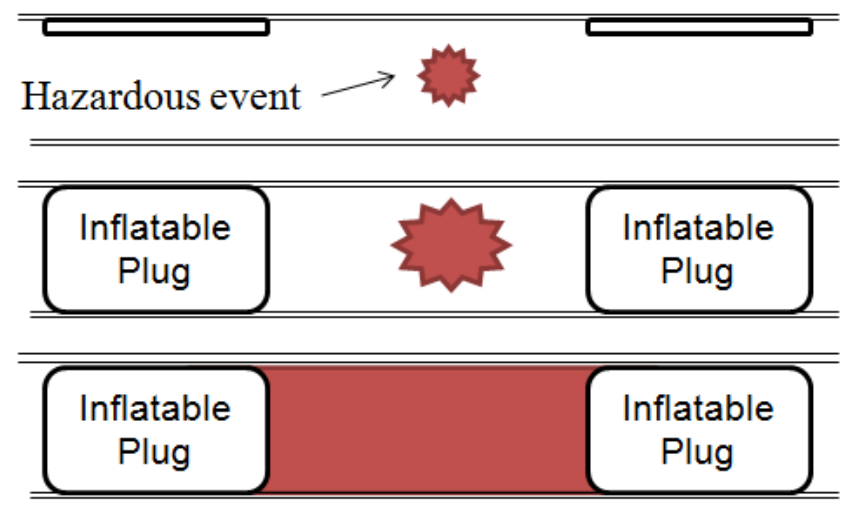

RTP Concept - Deployment Sequence:

1. Hazardous event happens.

2. Event is detected and it triggers

the deployment of inflatable plugs.

3. Threat containment.

Figure 1.13: Schematic of RTP deployment [14-15].

\subsection{Advantages and Disadvantages of Inflatable Structures for Tunnel Protection}

Just like in any structural configuration, the use of inflatable structures for tunnel protection has both advantages and disadvantages. Among the advantages, confined inflatable structures can be formed and self-supported with relatively low pressure and built to be flexible to cover irregular curved surfaces as demonstrated in [14-15]. Inflatable structures can be not only flexible for transportation and packing but can also be strong enough to be able to withstand aggressive loads like blasting, flooding, fire, or noxious substances when deployed and fully inflated. Unlike solid and rigid structures, inflatable structures offer great flexibility, are relatively easy to pack, transport and deploy when needed. Moreover, the inflatable structure can be activated remotely and have low or no human intervention for self-deploying.

Among the disadvantages, and depending on the particular application, the shape of the inflatable structure may not be maintained consistently rigid and a high 
energy consumption may be needed to maintain its shape and therefore its sealing effectiveness. Some instances may require anchoring points which could induce stress concentrations that can severely damage the structural membrane or other critical components. These anchoring points are mostly needed to restrain the structure during deployment; otherwise, the inflatable structures will be dislocated or misplaced during the inflation and pressurization process which can severely undermine its sealing effectiveness. Some applications may require high inflation pressures which depending on the design, they may require high or very high fabric strengths. This constraint may lead to heavier and more expensive solutions.

\subsection{Motivation of this Study}

Since 2008, WVU has worked in the development of RTP systems and conducted several validation tests of full and reduced scale prototypes of confined inflatable structures. Tests were performed using specially built testing facilities designed to simulate flooding in rail transportation tunnels [14-15, 28-29] (Figure 1.14). However, testing especially at full scale, either in an actual tunnel or in the specially built testing facilities is a very complex and expensive demanding task. The validation tests performed at full scale demonstrated that it can take several iterations to achieve acceptable results which cannot be accurately predicted in advance. Therefore, the development of numerical models of these tests using Finite Element Analysis (FEA) becomes imperative in order to: first, reproduce experimental results using different prototypes at different scales; and second, use the calibrated models as predicting tool that can anticipate the outcome of further experiments and eventually reduce its number due to the intrinsic operational complexity and associated costs. Calibrated models can also be used for parametric studies and design optimization that otherwise would be very costly to implement at experimental level. 

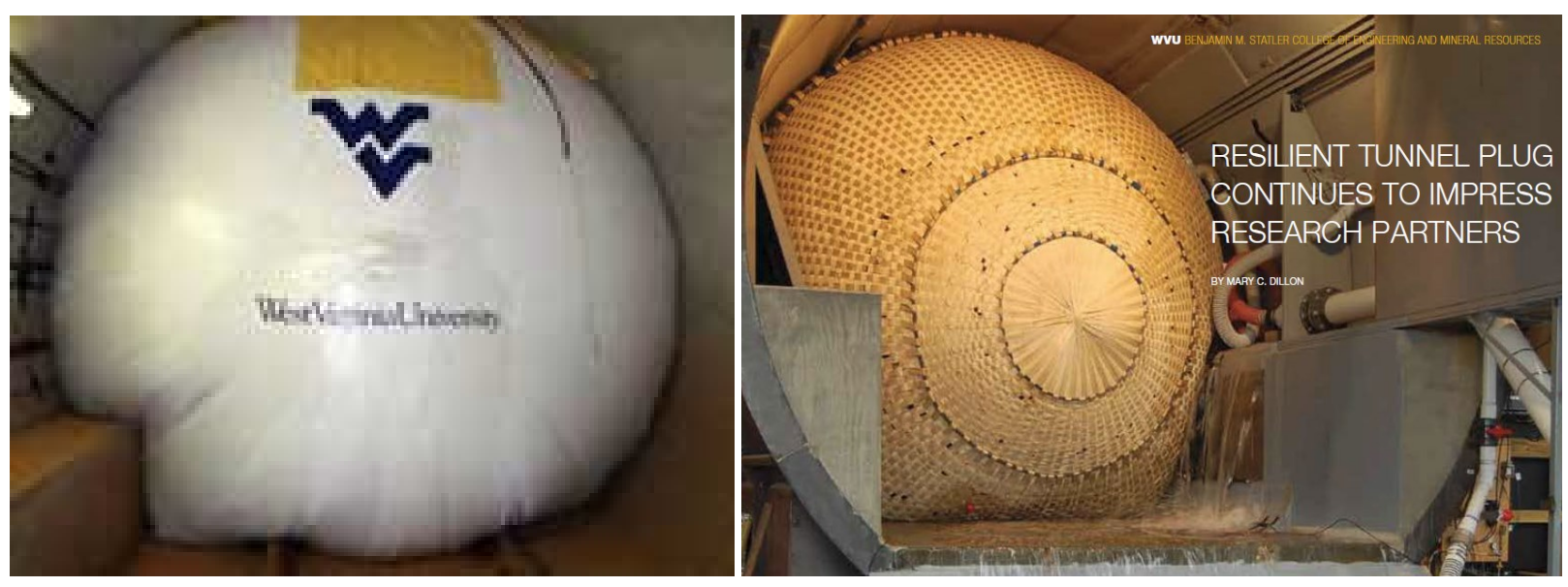

Figure 1.14: Plugs tested in an actual tunnel in 2008 (left) [14-15, 28], and in testing facilities at WVU in 2012 (right) [29].

\subsection{Objectives of this Study}

The main objectives of this study are:

1. Define a modeling strategy to identify necessary material properties and to devise ways to correlate experimental results with numerical results obtained from tests and models at different scales.

2. Create Finite Element Models of the RTP system to simulate:

a. The structural components of the inflatable plug

b. The folding of the inflatable

c. The deployment of the inflatable under confined conditions

d. The interaction of the inflatable with different elements of the confining surface and the global and local conformity to those elements

e. The stability of the inflatable to withstand different loading configurations and friction characteristics of the confining surface

3. Compare numerical results with available experimental results in order to adjust modeling parameters and improve the predicting capability of the models.

\subsection{Outline}

This dissertation is divided into eight chapters and begins with the problem statement, literature review, and objectives of this research presented in Chapter 1. 
Chapter 2 explains the fundamental theories of finite element method relevan to this work as well as the finite element algorithms used to perform FEA calculations implemented in this work.

The literature review of membrane elements is presented in Section 2 to describe the characteristic of membrane elements, especially when the elements are used for modeling of inflatable structures.

Chapter 3 presents a preliminary Finite Element model of a quarter-scale prototype used to get key parameters that are initially calibrated for comparing numerical and experimental results. Stress evaluations, mesh convergence studies, equivalent material properties evaluation, simplified deployment approach, and slippage evaluation are presented in this chapter.

The geometrical properties and set-up of the FE model of a full scale prototype are presented in Chapter 4 . Chapter 5 presents the modeling of folding and packing the inflatable plug. The deployment simulation consists of several main factors such as characteristic of membrane elements, folding process, influences of dynamic relaxation, and inflation algorithms. The procedure of placement and dynamic relaxation is explained in Chapter 6. Chapter 7 presents the inflation algorithms as well as details of the advanced deployment model and compares both numerical and experimental results. Chapter 8 presents the slippage test results of the full scale FE model. Finally, Chapter 9 presents summary conclusions obtained from the previous chapters and also provides recommendations for future work. Some of the chapters are complemented by Appendixes included at the end of this document. 


\section{Introduction to the Finite Element Method}

\subsection{Introduction}

The Finite Element Method (FEM) is widely used in both engineering and science applications. It was developed to deal with problem of stress analysis, fluid mechanics, heat transfer, vibrations, etc. through computational scheme [30]. An overview on how the method operates is as follows:

(i) The target geometry is divided into a finite number of pieces called elements.

(ii) The variation of the unknown variables at each element uses either interpolation or approximation functions to implement specific assumptions.

(iii) Nodes are the special element location where the approximated variation is eligible in terms of solution values.

(iv) The method generates an algebraic system of equations for unknown nodal values which approximate the continuous solution through the discretization process.

(v) The method can calculate and obtain a solution quite accurately due to the variability of element size, shape, and approximating scheme.

\subsection{General FEM Procedures}

Figure 2.1 shows a condition that assumes that the geometry of an arbitrary body under an unloaded condition $R_{o}$ initially has zero initial stress, $S_{o}$. The material is represented by the elastic constants, $C_{i j k l}$, the thermal expansion coefficient and temperature distribution are neglected for simplicity; it is also assumed that a body force, $b$, acts on the geometry, and displacements $u^{*}(x)$ act on a portion $\partial_{1} R$ or tractions, $t$, on a portion $\partial_{2} R$ of the boundary of $R$ [30]. 

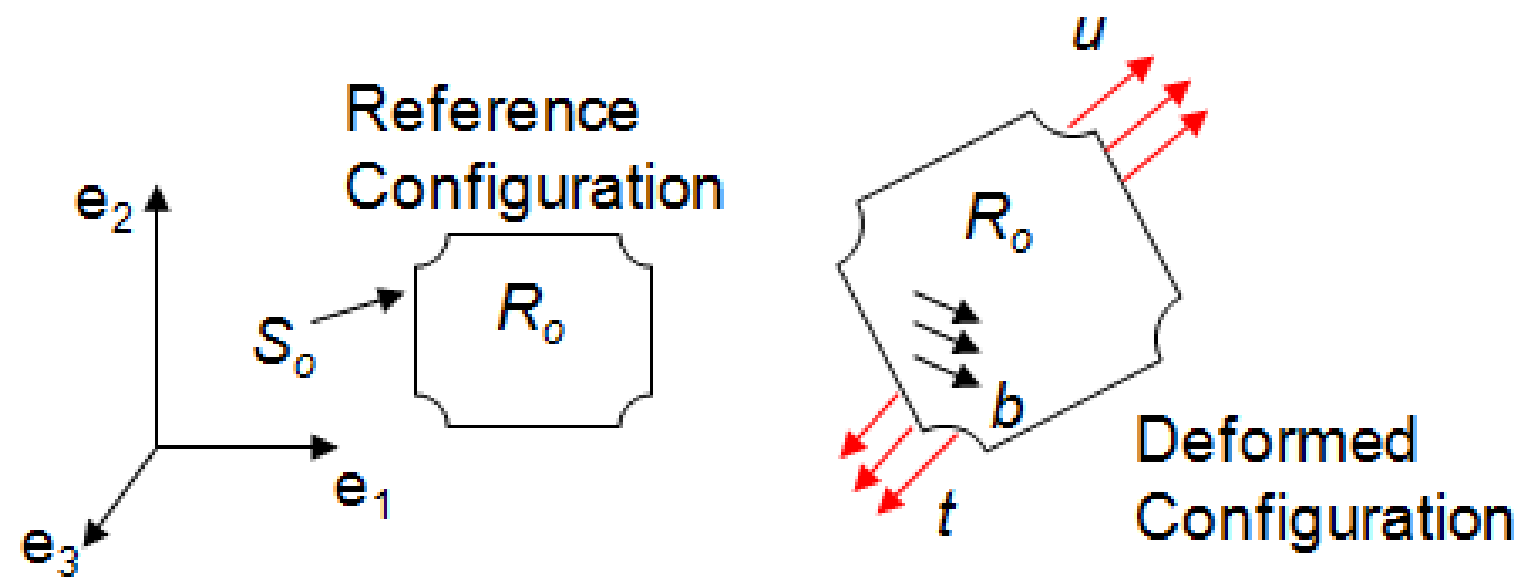

Figure 2.1: Conceptual Schematic of the finite element procedure.

To satisfy the governing equations, the component of displacements, strains, and stresses need to be calculated [30].

Strain-displacement equation, $\varepsilon_{i j}=\frac{1}{2}\left(\frac{\partial u_{i}}{\partial x_{j}}+\frac{\partial u_{j}}{\partial x_{i}}\right)$

Elastic stress-stain (constitutive) law, $\sigma_{i j}=C_{i j k l} \varepsilon_{k l}$

Equation of equilibrium, $\frac{\partial \sigma_{i j}}{\partial x_{i}}+b_{j}=0$

Boundary condition of stress and displacement,

$u_{i}=u_{i}^{*}$ on $\partial_{1} R ; \quad \sigma_{i j} n_{i}=t_{j}^{*}$ on $\partial_{2} R$

To derive the virtual work equation, the linear momentum equation balance is used for the derivation of the equation,

$\frac{\partial \sigma_{j i}}{\partial y_{j}}+\rho b_{i}=\rho \frac{d v_{i}}{d t}$

Substitute the virtual strain field equation into Equation 2.5,

$\delta \varepsilon_{i j}=\frac{1}{2}\left(\frac{\partial \delta v_{i}}{\partial x_{j}}+\frac{\partial \delta v_{j}}{\partial x_{i}}\right)$ 
The principle of virtual work can be expanded as follows,

$\int_{R} \sigma_{i j} \delta \varepsilon_{i j} d V_{0}-\int_{R} b_{i} \delta v_{i} d V_{0}-\int_{\partial_{2} R} t_{i} \delta v_{i} d A=0$

The virtual work equation can be used to derive the governing equation for the displacement field in a weak form by substituting equation 2.8 into equation 2.7 .

$\sigma_{i j} \delta \varepsilon_{i j}=C_{i j k l} \frac{\partial u_{i}}{\partial x_{j}} \frac{\partial \delta v_{i}}{\partial x_{j}}$

A general form of an interpolation equation can be expressed as,

$u_{i}(x)=\sum_{a=1}^{n} N^{a}(x) u_{i}^{a}$

where $x$ are the coordinates of an arbitrary point in the geometry and $N^{a}(x)$ are functions of position only. The virtual velocity can be derived in the same way,

$\delta v_{i}(x)=\sum_{a=1}^{n} N^{a}(x) \delta v_{i}^{a}$

Substituting equation 2.10 into 2.7 and rewriting the equation as

$\int_{R} C_{i j k l} \frac{\partial N^{b}(x)}{\partial x_{i}} u_{k}^{b} \frac{\partial N^{a}(x)}{\partial x_{j}} \delta v_{i}^{a} d V-\int_{R} b_{i} N^{a}(x) \delta v_{i}^{a} d V-\int_{\partial 2 R} t_{i}^{*} N^{a}(x) \delta v_{i}^{a} d A=0$

Since the interpolation functions are known, therefore, equation 2.11 can be rewritten as

$\left(K_{\text {aibk }} u_{k}^{b}-F_{i}^{a}\right) \delta v_{i}^{a}=0$

where $K_{a i b k}=\int_{R} C_{i j k l} \frac{\partial N^{a}(x)}{\partial x_{j}} \frac{\partial N^{b}(x)}{\partial x_{i}} d V$

and $F_{i}^{a}=\int_{R} b_{i} N^{a}(x) d V+\int_{\partial 2 R} t_{i}^{*} N^{a}(x) d A$

When the displacement satisfies with $u_{i}^{a}=u_{i}^{*}$ at the nodes,

$K_{\text {aibk }} u_{k}^{b}=F_{i}^{a} \quad \forall\{a, i\}: x_{k}^{a}$ not on $\partial_{1} R$

$u_{i}^{a}=u_{i}^{*}\left(x_{i}^{a}\right) \quad \forall\{a, i\}: x_{k}^{a}$ on $\partial_{1} R$

where $K$ is the stiffness matrix. 


\subsection{Modeling Tools}

A commercial finite element analysis software, Abaqus ${ }^{1}$, was used as solver of the general equations presented in the previous section. The models presented in this work were generated, solved and post-processed using different tools available within Abaqus.

Besides Abaqus, other commercial software and programming languages were exercised and interfaced with Abaqus for specific purposes. For instance, Altair HyperMesh $^{2}$ was used to renumber the nodes and elements during the pre-processing stage. Moreover, several subroutines files were generated in format of Fortran code, Python ${ }^{\mathrm{TM}}$, and Abaqus Scripting in order to facilitate the obtaining of simulation results during the post-processing stage.

Key features available in Abaqus 6.10 implemented in the models generated in this research are presented in the following sections.

\subsubsection{Element Types}

The element types which have been used in the finite element simulation of this research included membrane element, rigid body/rigid element, connector element, surface element, and solid element.

The membrane element is an element type which was used to define the woven layers of RTP in the FE model. The rigid body or rigid element was used to represent a part/section of a geometry which assumed to be rigid body such as fitting sections on the plug and tunnel wall in order to simplify the FE model.

The connector element was used to simulate the passive restraining mechanism in the FE model. On the other hand, the surface element was used to model the internal chamber walls in the FE model. The water in some of the slippage scenarios was modeled using solid element type.

\footnotetext{
${ }^{1}$ Abaqus is a finite element analysis (FEA) and computer-aided engineering (CAE) software package which has the capabilities in performing pre-processing (solid modeling), comprehensive FEA solving, and pro-processing (visual rendering). The software was acquired by Dassault Systèmes Simulia Corp. in 2005. Visit www.simulia.com for more details.

2 HyperMesh is one of the portfolios of HyperWorks, a CAE software product, belongs to Altair Engineering Inc.. HyperMesh is mainly used to perform comprehensive CAE pre- and post-processing tasks. Visit www.altair.com for more details.
} 


\subsubsection{Membrane Elements}

Green and Adkins [31] propounded the theory of elastic membranes by treating the problem as one of plane stress in a continuous form. The finite element method which defines an approximated continuum by a finite number of elements was used to formulate a consistent discrete model [32]. The formulation of finite number of discrete variables can be reduced to one which is the displacement of selected node points in the membrane [32].

Oden [33-34] and Kubitza [35] derived the general analysis of finite deformations of elastic membranes using linear displacement approximations and triangular finite elements when discussing the numerical analysis of nonlinear pneumatic structures. Oden and Sato [36] presented a consistent finite element formulation for the calculation of finite strain and large displacements in elastic membranes of general geometry.

Flores and Oñate [37-38] presented the improved membrane behavior of a thin shell triangular element which assumed to have a plane stress behavior with an additive decomposition of elastic and plastic strains and rotation-free degree of freedom through Lagrangian formulation.

Taylor et. al [39] used the right hand side of Cauchy-Green deformation tensor to develop the large deformation formulation of the three-node triangular membrane element where the deformation gradient is first constructed in term of nodal variables. Oñate et. al [40] used Lagrangian formulation to calculate the constant bending strains and linear membrane strains of inflatable structures. Three adjacent membrane elements which only have translational degrees of freedom were used to evaluate the strains in terms of the nodal displacements. This approach becomes a conventional method of finite element analysis as well as Abaqus to define the membrane elements.

In Abaqus, the element dimensionality is used to define membrane elements. General membrane elements are named as follows,

\section{$\underline{M} \underline{X D} \underline{Y}$}


where $\underline{\mathbf{M}}$ indicates membrane element, $\underline{\mathbf{X}}$ represents the dimensionality of the element, and $\underline{Y}$ shows the number of nodes in the element. For instance, M3D3 is a threedimensional, 3-node triangular membrane element and M3D4 is a three-dimensional, 4node quadrilateral membrane element [42-43]. Reduced integration (an optimization method uses less functions to solve the integral through Gaussian Quadrature) is available only to quadrilateral membrane elements [42-43].

Membrane elements have only three translational Degrees of Freedom (DOF) at each node [42-43]. Therefore, there are two significant characteristics in membrane elements: First, the membrane elements do not have bending stiffness. Second, they do not take bending moments into account because they are defined and handled as surface elements which can only transfer in-plane forces. Membrane elements usually are used to represent thin surfaces that provide strength in the plane of the element without bending stiffness [42-43].

The equilibrium of membrane elements begins with the definition of the virtual work contribution. The virtual work contribution in terms of the internal force can be expressed as [42-43],

$\delta W^{I}=\int_{V} \sigma: \delta \varepsilon d V$

where $\sigma$ indicates the Cauchy stress, $\delta \varepsilon$ is the virtual rate of deformation, and $V$ represents the current volume of the membrane [42-43]. The membrane stress components in the surface of the membrane are assumed to be nonzero $\left(\sigma_{3 i}=0\right)$, thus, the virtual work equation can be simplified as

$\delta W^{I}=\int_{V} \sigma_{\alpha \beta} \delta \varepsilon_{\alpha \beta} d V$

where $d V=t d A$ where $t$ is the current thickness and $A$ is the current area of the same element [42-43]. Since the Cauchy Stress is symmetric, the virtual work equation [42-43] can be rewritten as

$\delta W^{I}=\int_{V} \sigma_{\alpha \beta} \delta L_{\alpha \beta} d V$ 
where $\delta L_{\alpha \beta}=e_{\alpha} \frac{\partial \delta u}{\partial x} \cdot e_{\beta}=e_{\alpha} \frac{\partial \delta u}{\partial x_{\beta}}$ is a local orthonormal basis system $e_{i}$ that defines a basis on a surface in space through standard convention of ABAQUS, where $e_{1}$ and $e_{2}$ are in the surface of the element and $e_{3}$ is normal to the element by standard convention for a basis on a surface in space. When the Jacobian transformation is implemented to elaborate the integrands of virtual work [42-43], the equation can be written as

$d \delta W^{I}=\int_{A} t\left(\delta \varepsilon: D: d \varepsilon+\sigma:\left(\delta L^{T} \cdot d L-2 \delta \varepsilon \cdot d \varepsilon\right)\right) d A$

Since transverse stresses, $\sigma_{3 i}$, and transverse shear strains, $\varepsilon_{3 i}$, are assumed to be zero, and $\delta \varepsilon_{\alpha i} d \varepsilon_{i \beta}=\delta \varepsilon_{\alpha \gamma} d \varepsilon_{\gamma \beta}$, the first term of the integrand can be expressed as

$\delta \varepsilon_{\alpha \beta} D_{\alpha \beta \gamma \delta} d \varepsilon_{\gamma \delta}$

and the second term of integrand can be expanded as

$\sigma_{\alpha \beta}\left(\frac{\partial u}{\partial x_{\alpha}} \cdot \frac{\partial u}{\partial x_{\beta}}-\frac{1}{2}\left(e_{\gamma} \cdot \frac{\partial u}{\partial x_{\alpha}}+e_{\alpha} \cdot \frac{\partial u}{\partial x_{\gamma}}\right)\left(e_{\gamma} \cdot \frac{\partial u}{\partial x_{\beta}}+e_{\beta} \cdot \frac{\partial u}{\partial x_{\gamma}}\right)\right)$

An expanded equation of virtual work with consistent Jacobian transformations [42-43] can be rewritten as

$\sigma_{11} \frac{\partial \delta u}{\partial x_{1}} \cdot \frac{\partial d u}{\partial x_{1}}+\sigma_{22} \frac{\partial \delta u}{\partial x_{2}} \cdot \frac{\partial d u}{\partial x_{2}}+\sigma_{12}\left(\frac{\partial \delta u}{\partial x_{1}} \cdot \frac{\partial d u}{\partial x_{2}}+\frac{\partial \delta u}{\partial x_{2}} \cdot \frac{\partial d u}{\partial x_{1}}\right)-2 \sigma_{11} e_{1} \cdot \frac{\partial \delta u}{\partial x_{1}} e_{1} \cdot \frac{\partial d u}{\partial x_{1}}-2 \sigma_{22} e_{2} \cdot$

$\frac{\partial \delta u}{\partial x_{2}} e_{2} \cdot \frac{\partial d u}{\partial x_{2}}-\frac{1}{2}\left(\sigma_{11}+\sigma_{22}\right)\left(e_{2} \cdot \frac{\partial \delta u}{\partial x_{1}} e_{2} \cdot \frac{\partial d u}{\partial x_{1}}+e_{1} \cdot \frac{\partial \delta u}{\partial x_{2}} e_{1} \cdot \frac{\partial d u}{\partial x_{2}}+e_{1} \cdot \frac{\partial \delta u}{\partial x_{2}} e_{2} \cdot \frac{\partial d u}{\partial x_{1}}+e_{2} \cdot \frac{\partial \delta u}{\partial x_{1}} e_{1}\right.$.

$\left.\frac{\partial d u}{\partial x_{2}}\right)-\sigma_{12}\left(e_{1} \cdot \frac{\partial \delta u}{\partial x_{1}} e_{1} \cdot \frac{\partial d u}{\partial x_{1}}+e_{2} \cdot \frac{\partial \delta u}{\partial x_{1}} e_{2} \cdot \frac{\partial d u}{\partial x_{2}}+e_{1} \cdot \frac{\partial \delta u}{\partial x_{2}} e_{1} \cdot \frac{\partial d u}{\partial x_{1}}+e_{2} \cdot \frac{\partial \delta u}{\partial x_{2}} e_{2} \cdot \frac{\partial d u}{\partial x_{1}}+e_{1} \cdot \frac{\partial \delta u}{\partial x_{2}} e_{2}\right.$.

$\left.\frac{\partial d u}{\partial x_{2}}+e_{2} \cdot \frac{\partial \delta u}{\partial x_{1}} e_{1} \cdot \frac{\partial d u}{\partial x_{1}}+e_{1} \cdot \frac{\partial \delta u}{\partial x_{1}} e_{2} \cdot \frac{\partial d u}{\partial x_{1}}+e_{2} \cdot \frac{\partial \delta u}{\partial x_{2}} e_{1} \cdot \frac{\partial d u}{\partial x_{2}}\right)$

When the cross-sectional thickness changes with the Poisson's ratio as a function of the membrane strain in geometrically non-linear analyses, the linear elastic strain equation [42-43] can be expressed as

$\epsilon_{33}=-\frac{v}{1-v}\left(\epsilon_{11}+\epsilon_{22}\right),\left(\right.$ assuming in plane stress, $\left.\sigma_{33}=0\right)$ 
The relationship of thickness with respect to Poisson's ratio can be comprehended when the linear elastic strain is expressed in logarithmic form as [42-43],

$\ln \left(\frac{t}{t_{0}}\right)=-\frac{v}{1-v}\left(\ln \left(\frac{l_{1}}{l_{1}^{0}}\right)+\ln \left(\frac{l_{2}}{l_{2}^{0}}\right)\right)=-\frac{v}{1-v} \ln \left(\frac{A}{A^{0}}\right)$

$\frac{t}{t_{0}}=\left(\frac{A}{A_{0}}\right)^{-\frac{v}{1-v}}$

where $A$ is the area on the reference surface of the membrane. The deformation gradient can be written in $F=\partial x / \partial X$ and since there is no transverse shear [42-43], thus,

$F_{3 \alpha}=e_{3} \cdot \frac{\partial x}{\partial X_{\alpha}}=0$ and $F_{\alpha 3}=e_{\alpha} \cdot \frac{\partial x}{\partial X_{3}}=0$

Substituting the thickness change equation into the deformation gradient, the deformation gradient's direct out-of-plane component [42-43] can be expressed as

$F_{33}=\frac{1}{\left(F_{11} F_{22}-F_{12} F_{21}\right)^{\frac{v}{1-v}}}$

The two tangent vectors at the end of the increment can be used to calculate the deformation gradient through defining the derivative of the position that respects to the reference coordinates [42-43].

$\frac{\partial x^{n+1}}{\partial X_{\beta}}=\frac{\partial x^{n+1}}{\partial \xi_{\alpha}} \frac{\partial \xi_{\alpha}}{\partial x_{\beta}}$

where $\partial x^{n+1} / \partial \xi_{\alpha}$ is the interpolation shape function derived from nodal coordinates and $\partial \xi_{\alpha} / \partial X_{\beta}$ is the change of coordinate transformation on the reference geometry. The deformation gradient expressed and satisfied symmetry condition [42-43],

$F_{\alpha \beta}=e_{\alpha}^{n+1} \cdot \frac{\partial x^{n+1}}{\partial X_{\beta}}=F_{\beta \alpha}$

and the rotation angle is 
$\Delta \psi=\tan ^{-1}\left[\frac{\hat{F}_{21}-\hat{F}_{12}}{\hat{F}_{11}+\hat{F}_{22}}\right]$

\subsubsection{Rigid Body and Rigid Elements}

A rigid body can be a group of nodes, elements, or surfaces. The motion of this group is governed by the motion of a reference node called rigid body reference node. The rigid body elements can perform large rigid body motions but do not deform because the relative position of the nodes and elements of rigid body remains unchanged during the simulation [42-43].

Applying boundary conditions at the rigid body reference node defines the motion of a rigid body. The concentrated loads on nodes and distributed loads on elements of the rigid body generate the loads on a rigid body. Rigid bodies are able to connect to deformable elements at the nodes and to other rigid body with connector elements. Also, surfaces on rigid bodies are able to have contact interaction with other bodies in the model [42-43].

In Abaqus, rigid elements are named as below,

\section{$\underline{\mathbf{R}} \underline{\mathbf{X D}} \underline{\mathrm{K}}$}

where $\underline{\mathbf{R}}$ indicates rigid element, $\underline{\mathbf{X} \mathbf{D}}$ represents the dimensionality of element, and $\underline{\mathbf{K}}$ shows the number of nodes in the element. For instance, R3D3 is a three-dimensional, 3-node triangular rigid element and R3D4 is a three-dimensional, 4-node quadrilateral rigid element [42-43].

\subsubsection{Connector Elements}

Connector elements in Abaqus are used in two-dimensional, axisymmetric, and three-dimensional analyses for modeling a connection between two nodes. Each node can be connected to a deformable part, a rigid part, or not connected to any part [42-43]. The connector elements have relative local displacements and rotations and comprehensive kinematic and kinetic output [42-43]. Every connector element is constructed with two nodes only and the position and motion of the second node on each connector element are determined relative to the first node [42-43]. 
The convention for naming a connector element by using the acronym,

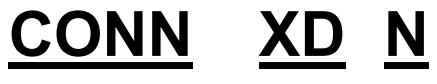

where $\underline{\text { CONN }}$ stands for connector element, $\underline{\text { XD }}$ represents the dimensional analyses and is available in 2D (two-dimensional) and 3D (three-dimensional), and the last $\underline{\mathbf{N}}$ in the term indicates the number of nodes, but the number of nodes is always 2 since all the connector elements only have two nodes at most [42-43]. There are two connector elements available in Abaqus depending on the dimensionality of the analysis: CONN2D2 is a 2-node connector element for two-dimensional and axisymmetric analyses and three-dimensional, and CONN3D2 is a 2-node connector element for three-dimensional analyses [42-43].

Among the translational connector elements, the AXIAL and CARTESIAN are the two connection types that include translational and rotational degrees of freedom at both node $a$ and node $b$ [42-43]. Connection type AXIAL (Figure 2.2) defines a discrete physical connection between two nodes where the relative displacement is used to measure the line separating the two nodes [42-43].

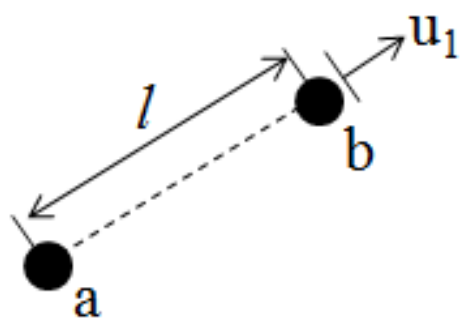

Figure 2.2: Connection Type AXIAL.

The AXIAL connection does not impose any constraint on relative motion. The distance between nodes $a$ and $b$ can be expressed as

$l=\left\|x_{b}-x_{a}\right\|$ 
The relative motion, $u_{1}$, acts along the connection line between two nodes and determines the change of the distance between the two nodes [42-43]. The relative motion can be defined as

$u_{1}=l-l_{0}$

where $l_{0}$ is the initial distance between nodes $a$ and $b$. From the classic spring force, the kinetic force [42-43] can be written as

$f_{\text {axial }}=f_{1} q$

where $f_{1}$ is the force at component 1 and $q$ is the coefficient of nominal length between node $a$ and $b$. The nominal length [42-43] can be defined as

$q=\frac{\left(x_{b}-x_{a}\right)}{\left\|x_{b}-x_{a}\right\|}$

Connection type CARTESIAN (Figure 2.3) defines the connection between two nodes by measuring the change in position of node $b$ in three local connection directions $\left\{e_{1}^{a}, e_{2}^{a}, e_{3}^{a}\right\}$ for node $a$ [42-43]. The local directions follow the rotation of node $a$ at node $a$. Connection type CARTESIAN does not constrain any relative motion [4243].

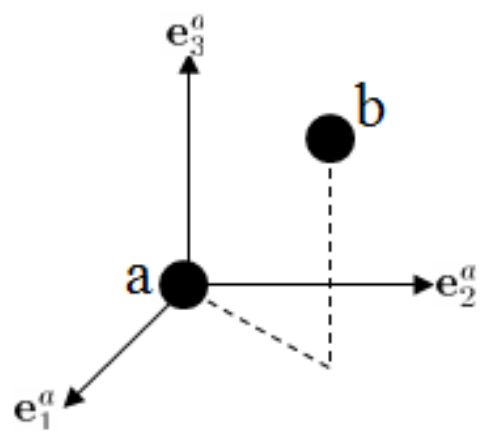

Figure 2.3: Connection Type CARTESIAN.

The position of node $b$ respects to node $a$ [42-43] can be expressed as

$x=e_{1}^{a} \cdot\left(x_{b}-x_{a}\right)$ 
$y=e_{2}^{a} \cdot\left(x_{b}-x_{a}\right)$

$z=e_{3}^{a} \cdot\left(x_{b}-x_{a}\right)$

and the available components of relative motion [42-43] can be derived as

$u_{1}=x-x_{0}$

$u_{2}=y-y_{0}$

$u_{3}=z-z_{0}$

The kinetic force can be expressed in terms of local connection directions [42-43] ,

$f_{\text {Cartesian }}=f_{1} e_{1}^{a}+f_{2} e_{2}^{a}+f_{3} e_{3}^{a}$

There are many types of effects that can be used to define the connector behavior [42-43] such as

- Elasticity: Defines the linear or nonlinear elastic effect on connector through relative motion dependently or independently using elastic stiffness (force or moment per relative displacement or rotation).

- Failure: Failure of specified components in the connection if a failure criterion is achieved.

- Friction: Defines the frictional effect on connector using the concept of Coulomb's friction (refer to Equation 2.72).

- Plasticity: Defines the plastic effect on connector using classical plasticity formulations.

- Damage: Performs the irreversible damage (degradation) on elastic, elasticplastic, force response in connector elements if relative forces or motions exceed critical values in a connection.

- Stops and locks: Defines stopping or locking effects on the connection using available components of relative motion or relative force within certain limit values. 
- Uniaxial behavior: Specifies the loading, unloading behavior, individual response in the tensile and compressive directions, nonlinear elastic behavior, damaged elastic behavior, or elastic-plastic behavior with permanent deformation on the connection.

\subsubsection{Surface Elements in Abaqus}

Surface elements are defined just like membrane elements, but with zero thickness [42-43]. Therefore, the surface elements do not have inherent stiffness, bending stiffness, or transverse stiffness [42-43] and the numerical equations of this type of element are shown in Section 2.3.1.1. Surface elements can be used to define rigid bodies, embedded in solid element, have mass per unit area, and transmit only inplane forces.

The convention for naming a general surface element by using the acronym,

\section{$\underline{\text { SF }} \underline{M} \underline{3 D} \underline{\mathbf{N}}$}

where $\underline{\mathrm{SF}}$ stands for surface element, $\underline{\mathrm{M}}$ indicates the membrane-like behavior, $\underline{\text { 3D }}$ represents the dimensional analyses and is available in 3D (three-dimensional) only, and the last $\underline{\mathbf{N}}$ in the term indicates the number of nodes and is available in 3-node (triangle), 4-node (quadrilateral), 6-node (high order triangle), and 8-node (high order quadrilateral) [42-43]. Reduced integration is available only to quadrilateral surface elements [42-43].

Surface elements are used to specify the surfaces under a constraint without structure properties. Also, surface elements are used as a model in the form of a mass per unit area. For instance, the mass of fluid in a tank can be represented by a model of surface elements with mass per unit area [42-43].

\subsubsection{Solid (Continuum) Elements}

In Abaqus, solid elements are the standard volume elements. Solid elements can be used to define homogeneous material or define the laminated composite solid with 
several layers of different materials. Quadrilaterals and hexahedral (solid) elements are more accurate if not distorted compared to triangular and tetrahedral (solid) elements, but the latter are less sensitive to distortion [42-43].

Solid elements are available for linear analysis, nonlinear analyses (including contact, plasticity, and large deformations), stress, heat transfer, acoustic, coupled thermal-stress, coupled pore fluid-stress, piezoelectric, electromagnetic, and coupled thermal-electrical analyses [42-43].

A three-dimensional solid (continuum) element with reduced integration can be named as follows:

\section{$\underline{\mathrm{C}} \quad \underline{3 \mathrm{D}} \underline{\mathrm{N}} \underline{\mathrm{R}}$}

where $\underline{\mathbf{C}}$ stands for continuum stress/displacement, $\underline{\text { 3D }}$ represents the dimensional analyses in 3D (three-dimensional), and the $\underline{\mathbf{N}}$ in the term indicates the number of nodes and is available in 4-node (linear tetrahedron), 6-node (linear triangular prism), 8node (linear brick), 10-node (quadratic tetrahedron), 15-node (quadratic triangular prism), and 20-node (quadratic brick) [42-43]. The $\underline{\mathbf{R}}$ indicates the reduced integration option. Hexahedral elements are recommended in three-dimensional analyses due to high result accuracy for minimum cost [42-43].

All the solid elements in Abaqus are written in term of finite-strain components which allow for finite strain and rotation in large displacement analysis [42-43]. The general governing equations of displacement, strain, and stress have been discussed in Session 2.2.

\subsection{Material Properties}

The structural membrane of the inflatable structures analyzed in this work is modeled by an equivalent single layer membrane that is structurally representative of the actual tri-layer system used in testing prototypes. 


\subsubsection{Fabric Material Behavior}

The fabric material model is considered orthotropic and non-linear. When a fabric material model created according to a woven fabric that is made up of yarns in the fill and warp direction, the mechanical response of the woven fabric can be acquired [4243]. The fabric material model also can be represented by the material that has two non-orthogonal directions to each other. The changes between angle of the shear strain and the nominal strains along the yarn directions is a function that can be defined as local fabric stresses in the fabric material model [42-43]. Figure 2.4 illustrates the orthogonal basis of material and the local directions.

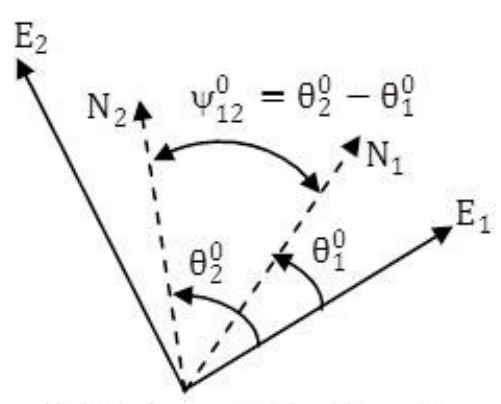

(a) Reference Configuration

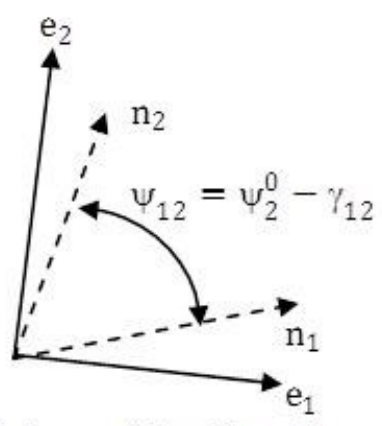

(b) Deformed Configuration

Figure 2.4: Fabric Kinematics [42-43].

The Cauchy stress, $\sigma$, can be calculated with respect to nominal stress, $T$, the work coupled with nominal strain and the Cauchy stress equation can be written as,

$$
\begin{aligned}
J \sigma= & \lambda_{1} T_{11} n_{1} n_{1}+\lambda_{2} T_{22} n_{2} n_{2}+ \\
& T_{12} \csc \left(\psi_{12}\right)\left(n_{1} n_{2}+n_{2} n_{1}\right)-T_{12} \cot \left(\psi_{12}\right)\left(n_{1} n_{1}+n_{2} n_{2}\right)
\end{aligned}
$$

where $J$ is the volumetric Jacobian, $\lambda$ is yarn stretch value, $n$ is nominal strain along yarn direction, and $\psi$ is the change between angle of two yarn direction (from reference to deformed configuration) [42-43].

The picture-frame test method was used to study the shear response of fabric [60]. Figure 2.5 shows schematically the setup of a picture-frame test where $L_{0}$ is the length of the picture-frame side, and $\psi_{12}$ is the angle between the yarn directions [42- 
43]. The four sides of the fabric specimen are constrained completely so that the length cannot be changed when the frame elongates and the angle between yarn directions changes under the action of the force, $F$ [42-43].
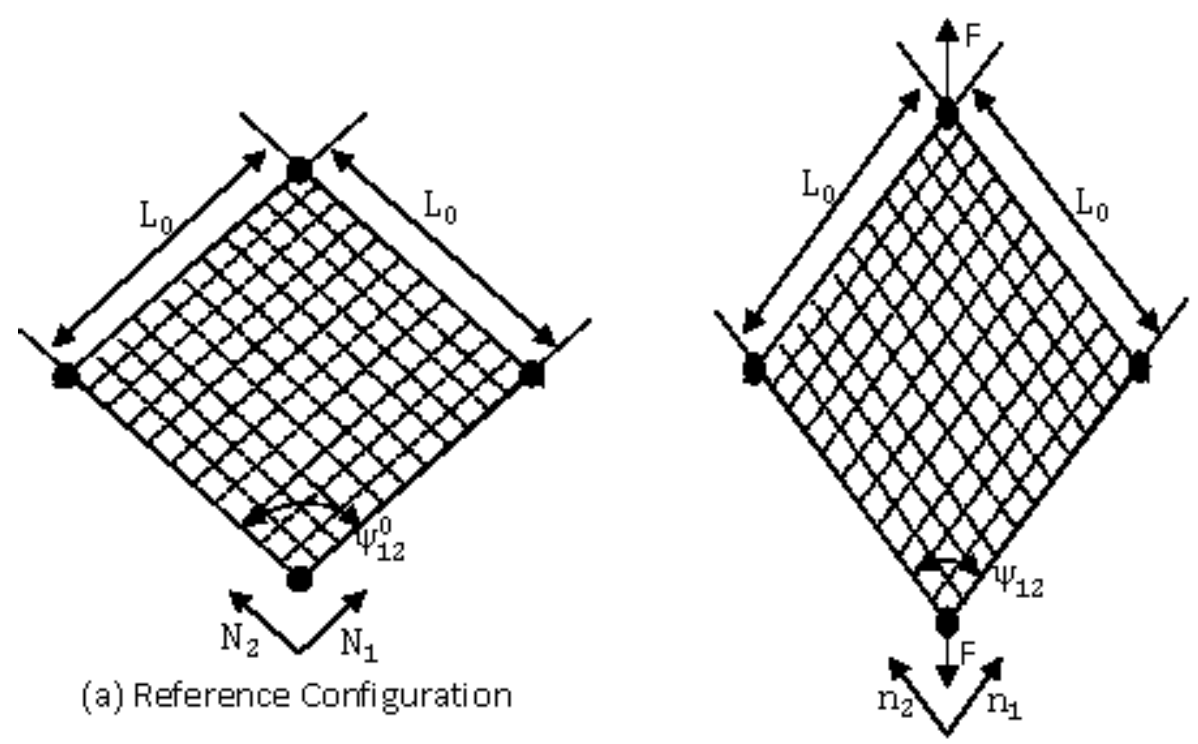

(b) Deformed Configuration

Figure 2.5: Picture-frame shear test [42-43].

The angle between yarn directions changed from reference to deformed configuration under force, $F$. The equation of nominal shear stress $T_{12}$ with respect to $F$ can be expressed as follows [42-43]:

$T_{12}=\left(\frac{F L_{0}}{v_{0}}\right) \sin \left(\frac{\psi_{12}}{2}\right)$

where $v_{0}$ is the initial volume of the fabric specimen. The fabric engineering shear strain can be defined through changes of angle between the yarn directions [42-43],

$\gamma_{12}=\psi_{12}^{0}-\psi_{12}$

\subsubsection{Material Damping}

Abaqus uses Rayleigh damping to introduce damping to the models that do not have energy dissipation sources. For analyses cases related to direct-solution steadystate dynamic and subspace-based steady-state dynamic, Rayleigh damping can be 
used to predict quantitatively accurate results, especially in the natural frequencies [4243].

Rayleigh damping can be described as a convenient generalized concept to damp lower (mass-dependent) and higher (stiffness-dependent) frequency range behaviors [42-43]. The Rayleigh damping, $\xi_{i}$, can be defined as follows:

$\xi_{i}=\frac{\alpha_{R}}{2 \omega_{i}}+\frac{\beta_{R} \omega_{i}}{2}$

where $\alpha_{R}$ is mass proportional damping or friction damping (unit of $1 /$ time), and $\beta_{R}$ is stiffness proportional damping (unit of time), and $\omega_{i}$ is the natural frequency of mode $i$ (unit of rad/sec) [42-43]. The damping factors alpha $(\alpha)$ and beta $(\beta)$ are assigned as part of the material properties. In most of the cases, the friction damping can be ignored [42-43]. The Rayleigh damping ratio equals to half of internal damping loss factor, $\eta_{i}$, thus the equation of stiffness proportional damping, $\beta_{R}$, can be rewritten as shown below [42-43].

$\frac{\eta_{i}}{2}=\frac{\beta_{R} \omega_{i}}{2}$

$\eta_{i}=\beta_{R} \omega_{i}$

$\beta_{R}=\frac{\eta_{i}}{\omega_{i}}$

\subsection{Properties of Fluid Models}

In Abaqus, the surface-based fluid-filled cavities under hydrostatic conditions can be modeled and calculated using surface definition through the coupling of the deformation of the fluid-filled structure and the enforcing pressure on the boundary of the fluid cavity [42-43]. The compressibility of a fluid can be defined through Mach Number below,

$M=\frac{v}{a}$ 
where $v$ is velocity of the source relative to the fluid and $a$ is the speed of sound in the fluid [42-43].

In this study, the hydraulic fluid that represents water is labeled as incompressible model and the pneumatic fluid that represents air is labeled as compressible model [42-43]. The pressure of a fluid (compressible or incompressible) can be calculated from the fluid volume, $\bar{V}$, which is a function of the fluid pressure, $p$; the fluid temperature, $\theta$; and the fluid mass, $m$, in the cavity:

$\bar{V}=\bar{V}(p, \theta, m), \quad \frac{d \bar{V}}{d p}=-\frac{m}{\rho_{R} K}$

where $K$ is fluid bulk modulus, $\rho_{R}$ is the reference density of fluid at zero pressure and initial temperature, and $m$ is total fluid mass [42-43]. The fluid volume, $\bar{V}$, should equal to actual volume of the cavity fluid, $V$, [42-43] thus,

$V-\bar{V}=0$

When the fluid is assumed to perform like an ideal gas, the density of the fluid in the cavity can be defined as [42-43],

$\rho(p, \theta)=\frac{p+p_{A}}{R\left(\theta-\theta_{A}\right)}$

where $R$ represents the gas constant, $\theta_{A}$ is the temperature at absolute zero, and $p_{A}$ indicates the ambient pressure [42-43].

The expanded virtual work equation with respect to cavity pressure can be written as follows,

$\delta \Pi^{*}=\delta \Pi-p \delta V-\delta p(V-\bar{V})$

where the expanded virtual work equation, $\delta \Pi^{*}$, equals to the virtual work equation (without considering the cavity), $\delta \Pi$, releases energy from the fluid when it increases the cavity volume [42-43]. Equation 2.50 also indicates the structural displacements and fluid pressure as primary variables for a mixed formulation [42-43]. The rate of change 
of the expanded virtual work equation can be extended by implementing the pressure load stiffness, $-p d \delta V$, and the volume-pressure compliance of the fluid, $d \bar{V} / d p$ [42-43].

$$
\begin{aligned}
d \delta \Pi^{*} & =d \delta \Pi-p d \delta V-d p \delta V-(d V-\overline{d V}) \delta p \\
& =d \delta \Pi-p d \delta V-d p \delta V-d V \delta p+\frac{d \bar{V}}{d p} d p \delta p
\end{aligned}
$$

Again, the expanded virtual work equation equals the sum of right-hand expression since the pressure for all the surface facets (or elements) in the cavity is uniformly distributed [42-43]. Thus,

$$
\begin{aligned}
\delta \Pi^{*} & =\delta \Pi-p \sum_{e} \delta V^{e}-\delta p\left[\sum_{e} V^{e}-\sum_{e} \bar{V}^{e}\right] \\
& =\sum_{e}\left[\delta \Pi^{e}-p \delta V^{e}-\delta p\left(V^{e}-\bar{V}^{e}\right)\right]
\end{aligned}
$$

Likewise, the volume of fluid element of each facet individually can be calculated since the temperature for all cavity facets is considered to be constant [42-43].

$\bar{V}^{e}=\bar{V}^{e}\left(p, \theta, m^{e}\right)$

where $\mathrm{m}^{\mathrm{e}}$ indicates as element mass. However, the total fluid volume defines the volume of the cavity and vice versa in the solution [42-43].

\subsubsection{Pneumatic Fluid}

Jetteur et. al [44] and Bonet et. al [118] used the Boyle's law (changes in volume) to determine the changes in air pressure when performing a FEA of air supported membrane structures. The fluid pressure equation of Boyle's law for a pneumatic system, at either isothermal or adiabatic stage, can be defined as the changes of initial pressure with respect to the ratio of changed volume $[53,118]$,

$p=p_{o} \frac{V_{o}}{v}=$ constant

where $p_{o}$ is the initial pressure, $V_{o}$ represents the initial volume, and $v$ is the current volume. The pressure is constant over the entire volume. 
On the other hand, Rumpel et. al [54-55] introduced the Poisson's law to specify the physical behavior of the gas in their models,

$p v^{k}=P_{o} V_{o}^{k}=$ constant

where $k$ is the isentropic constant, $P_{o}$ and $V_{o}$ are initial pressure and volume, respectively.

The ideal gas law is commonly used as one of the important elements in inflation algorithm in automotive airbag simulation. In Abaqus, the pneumatic fluid is defined as ideal gas [43] and further explanation of ideal gas is presented in Chapter 7.

\subsubsection{Hydraulic Fluid}

On the other hand, the fluid pressure equation for a hydraulic system is defined through Hooke's law. The fluid pressure in hydraulic models is determined by the change of volume and bulk modulus of the fluid [54].

$p=-K \frac{v-V_{o}}{V_{o}}$

where $K$ is the bulk modulus of the fluid, $V_{o}$ is the initial volume, and $v$ is the current fluid volume.

\subsubsection{Surface-based Fluid Cavity Algorithm}

Abaqus/Explicit uses the Gauss integral theorem to calculate the volume of fluid within the inflatable structure. When Abaqus calculates the volume for a fluid cavity, a reference node needs to be defined. The cavity reference node is associated with a surface facet geometry in order to create the fluid cavity within a completely enclosed cavity boundary (Figure 2.6) [42-43]. 


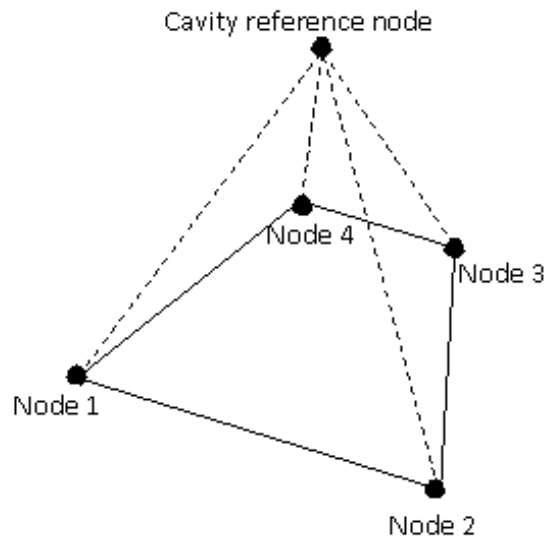

Figure 2.6: Cavity reference node of the fluid cavity [42-43].

To calculate the volume, $V^{e}$, of each element, the process begins with the coordinates of a point. The coordinates of each point on the pyramid element are defined by

$X=\sum_{N} N^{N}(g, h) X^{N}$

$X$ is the summation of all the nodes on the base, where $N^{N}$ are the interpolation functions with parametric coordinates $g$ and $h$, and $X^{N}$ are the nodal coordinates [42-43]. The Jacobian on the surface for three-dimensional elements is taken into account as

$\frac{\partial X}{\partial g}=\sum_{N} \frac{\partial N^{N}}{\partial g} X^{N}, \quad \frac{\partial X}{\partial h}=\sum_{N} \frac{\partial N^{N}}{\partial h} X^{N}$

An infinitesimal area of the element face, $d A$, is multiplied to the normal to the element face, $n$, to obtain,

$n d A=\left(\frac{\partial X}{\partial g} \times \frac{\partial X}{\partial h}\right) d g d h$

The infinitesimal area associated with the infinitesimal volume, $d V$, and expressed as

$d V=\frac{1}{3}\left(X_{R}-X\right) \cdot n d A$

where $X_{R}$ is the coordinate points of the cavity reference node. The volume of the element, $V^{e}$, can then be obtained. For a quadrilateral base this yields 
$V^{e}=\int_{V^{e}} d V=\int_{-1}^{+1} \int_{-1}^{+1} \frac{1}{3}\left(X_{R}-X\right) \cdot\left(\frac{\partial X}{\partial g} \times \frac{\partial X}{\partial h}\right) d g d h$

However, the triangular base has different of integration boundaries. Subsituting the relative position, $\bar{X}=X-X_{R}$, into previous equation,

$V^{e}=\int_{-1}^{+1} \int_{-1}^{+1}-\frac{1}{3} \bar{X} \cdot\left(\frac{\partial \bar{X}}{\partial g} \times \frac{\partial \bar{X}}{\partial h}\right) d g d h$

And the variation in the element volume can be written as

$\delta V^{e}=\int_{-1}^{+1} \int_{-1}^{+1}-\frac{1}{3}\left[\delta \bar{X} \cdot\left(\frac{\partial \bar{X}}{\partial g} \times \frac{\partial \bar{X}}{\partial h}\right)+\bar{X} \cdot\left(\frac{\partial \delta \bar{X}}{\partial g} \times \frac{\partial \bar{X}}{\partial h}+\frac{\partial \bar{X}}{\partial g} \times \frac{\partial \delta \bar{X}}{\partial h}\right)\right] d g d h$

The equivalent forces will be considered as the effect of the pressure on sides of the pyramid element since the equation above contributes to the volume change due to variation in the these sides. The side of the adjacent pyramid element will balance the pressure on the sides. Therefore, taking the pressure on the base of pyramid into account, the calculation of such pressure is addressed [42-43]. The equation can be derived by using partial integration as follows,

$\delta V_{b}^{e}=\int_{-1}^{+1} \int_{-1}^{+1}-\delta \bar{X} \cdot\left(\frac{\partial \bar{X}}{\partial g} \times \frac{\partial \bar{X}}{\partial h}\right) d g d h-\int_{-1}^{+1}\left[\bar{X} \cdot\left(\delta \bar{X} \times \frac{\partial \bar{X}}{\partial h}+\bar{X} \times \frac{\partial \delta \bar{X}}{\partial h}\right)\right]_{g=-1}^{g=1} d h-$

$\int_{-1}^{+1}\left[\bar{X} \cdot\left(\frac{\partial \delta \bar{X}}{\partial g} \times \bar{X}+\frac{\partial \bar{X}}{\partial g} \times \delta \bar{X}\right)\right]_{h=-1}^{h=1} d g$

The equation can be simplified by eliminating the last two integrals which represent the contributions on the sides of the pyramid; a simpler equation is obtained and as shown below,

$\delta V_{b}^{e}=\int_{-1}^{+1} \int_{-1}^{+1}-\delta \bar{X} \cdot\left(\frac{\partial \bar{X}}{\partial g} \times \frac{\partial \bar{X}}{\partial h}\right) d g d h$

The second variation of the volume can be expressed as,

$d \delta V_{b}^{e}=\int_{-1}^{+1} \int_{-1}^{+1}-\frac{1}{3}\left[\delta \bar{X} \cdot\left(\frac{\partial d \bar{X}}{\partial g} \times \frac{\partial \bar{X}}{\partial h}+\frac{\partial \bar{X}}{\partial g} \times \frac{\partial d \bar{X}}{\partial h}\right)+d \bar{X} \cdot\left(\frac{\partial \delta \bar{X}}{\partial g} \times \frac{\partial \bar{X}}{\partial h}+\frac{\partial \bar{X}}{\partial g} \times \frac{\partial \delta \bar{X}}{\partial h}\right)+\bar{X}\right.$.

$\left.\left(\frac{\partial \delta \bar{X}}{\partial g} \times \frac{\partial d \bar{X}}{\partial h}+\frac{\partial d \bar{X}}{\partial g} \times \frac{\partial \delta \bar{X}}{\partial h}\right)\right] d g d h$ 
For three-dimensional elements, the first and second variations can be generated in the same way and the integrations can be derived analytically. Take a shell element with 4 nodes for example, the above equations can be established in integral form,

$V^{e}=\frac{1}{12}\left[\left(\bar{X}^{3}-\bar{X}^{1}\right) \cdot\left(\bar{X}^{2} \times \bar{X}^{4}\right)+\left(\bar{X}^{4}-\bar{X}^{2}\right) \cdot\left(\bar{X}^{3} \times \bar{X}^{1}\right)\right]$

where $\bar{X}^{N}, N=1,2,3$, and 4 represent the relative nodal coordinates. The first variation of the volume, $\delta V_{b}^{e}$, that only involves the base of pyramid element can be expressed as

$\delta V_{b}^{e}=\frac{1}{12}\left[\delta \bar{X}^{1} \cdot\left(\bar{X}^{2}-\bar{X}^{4}\right) \times\left(2 \bar{X}^{1}-\bar{X}^{2}-\bar{X}^{3}\right)+\delta \bar{X}^{2} \cdot\left(\bar{X}^{3}-\bar{X}^{1}\right) \times\left(2 \bar{X}^{2}-\bar{X}^{3}-\bar{X}^{4}\right)+\right.$

$\left.\delta \bar{X}^{3} \cdot\left(\bar{X}^{4}-\bar{X}^{2}\right) \times\left(2 \bar{X}^{3}-\bar{X}^{4}-\bar{X}^{1}\right)+\delta \bar{X}^{4} \cdot\left(\bar{X}^{1}-\bar{X}^{3}\right) \times\left(2 \bar{X}^{4}-\bar{X}^{1}-\bar{X}^{2}\right)\right]$

and the second variation of the volume, $d \delta V^{e}$, is

$$
\begin{aligned}
& d \delta V^{e}=\frac{1}{12}\left[\left(\delta \bar{X}^{2} \times d \bar{X}^{1}-\delta \bar{X}^{1} \times d \bar{X}^{2}\right) \cdot\left(\bar{X}^{3}-\bar{X}^{4}\right)+\left(\delta \bar{X}^{3} \times d \bar{X}^{2}-\delta \bar{X}^{2} \times d \bar{X}^{3}\right)\right. \\
& \left(\bar{X}^{4}-\bar{X}^{1}\right)+\left(\delta \bar{X}^{4} \times d \bar{X}^{3}-\delta \bar{X}^{3} \times d \bar{X}^{4}\right) \cdot\left(\bar{X}^{1}-\bar{X}^{2}\right)+\left(\delta \bar{X}^{1} \times d \bar{X}^{4}-\delta \bar{X}^{4} \times d \bar{X}^{1}\right) \\
& \left(\bar{X}^{2}-\bar{X}^{3}\right)+\left(\delta \bar{X}^{1} \times d \bar{X}^{3}-\delta \bar{X}^{3} \times d \bar{X}^{1}\right) \cdot\left(\bar{X}^{2}-\bar{X}^{4}\right)+\left(\delta \bar{X}^{2} \times d \bar{X}^{4}-\delta \bar{X}^{4} \times d \bar{X}^{2}\right) \\
& \left.\left(\bar{X}^{3}-\bar{X}^{1}\right)\right]
\end{aligned}
$$

The fluid-filled cavity can be used to model the behavior of hydraulic or pneumatic fluid. The pneumatic model can be used to simulate nearly incompressible or fully incompressible fluid behavior through definition of ideal gas. On the other hand, the compressibility of hydraulic model can be defined by a bulk modulus [42-43].

Since the inertia of the fluid cavity or fluid exchanged between cavities is not taken into account during simulation, a reasonable representation of the distributed fluid mass needs to be added in order to define the effect of inertia [42-43]. Due to the pressure of fluid cavity is assumed to be uniform (equation 2.52) and the calculation of nodal displacement starts instantaneously when the analysis pressure begins to react on the elements. That restricts the fluid cavity from modeling any pressure gradientdriven fluid motions within a meshed structure [42-43]. 


\subsection{Friction and Contact Properties}

The coefficient of frictional (shear) stress under contact pressure interfacing with contact bodies was calculated through the Coulomb's law of friction [42-43]. The critical shear stress of the sliding surfaces was defined as a fraction of contact pressure between the surfaces [42-43].

$\tau_{\text {crit }}=\mu p$

where $\tau_{\text {crit }}$ is the critical shear stress, $\mu$ is the friction coefficient, and $p$ is the contact pressure. $\mu$ was assumed to be isotropic friction in this study [42-43]. Sticking state happens when two contacting surfaces interface with each other and generate shear stress up to certain magnitude before begin sliding relative one to another [42-43].

\subsection{Pressure Types}

\subsubsection{Uniform Pressure}

The external virtual work can be expressed in terms of pressure as

$\delta W^{E}=\int_{A} \delta \boldsymbol{u} \cdot p \boldsymbol{n} d A$

where $A$ is the surface which the pressure is applied on the normal direction, $n$, pointing into the material and to the surface, $\delta u$ is the virtual displacement field, and $p$ is the pressure [42-43].

When the pressure load stiffness is applied on a surface in a three-dimensional space, the expression $\boldsymbol{n} d A$ can be expanded as follows,

$n d A=\frac{\partial x}{\partial g} \times \frac{\partial x}{\partial h} d g d h$

where $x$ are the current coordinates of a point on the surface and both $g$ and $h$ are the surface parametric coordinates [42-43]. Through the cross product, the surface parametric coordinates defines the correct sign of $n$ [42-43]. The external virtual work can be rewritten as, 
$-\delta W^{E}=-\int_{g, h} p \delta u \cdot\left(\frac{\partial x}{\partial g}+\frac{\partial x}{\partial h}\right) d g d h$

and the load stiffness matrix can be obtained from the following expression [42-43],

$-d \delta W^{E}=-\int_{g, h} p \delta u \cdot\left(\frac{\partial d u}{\partial g} \times \frac{\partial x}{\partial h}+\frac{\partial x}{\partial g} \times \frac{\partial d u}{\partial h}\right) d g d h$

where $d x=d u$ for a solid model.

\subsubsection{Hydrostatic Pressure}

A schematic of the hydrostatic load scenario is presented in Figure 2.7. A breach is assumed to occur in the proximity of a tunnel segment. The breach can produce a leak that can flood the interior of the tunnel. The function of the inflatable plug is to avoid the propagation of the flooding by containing the water within a determined segment of the tunnel.

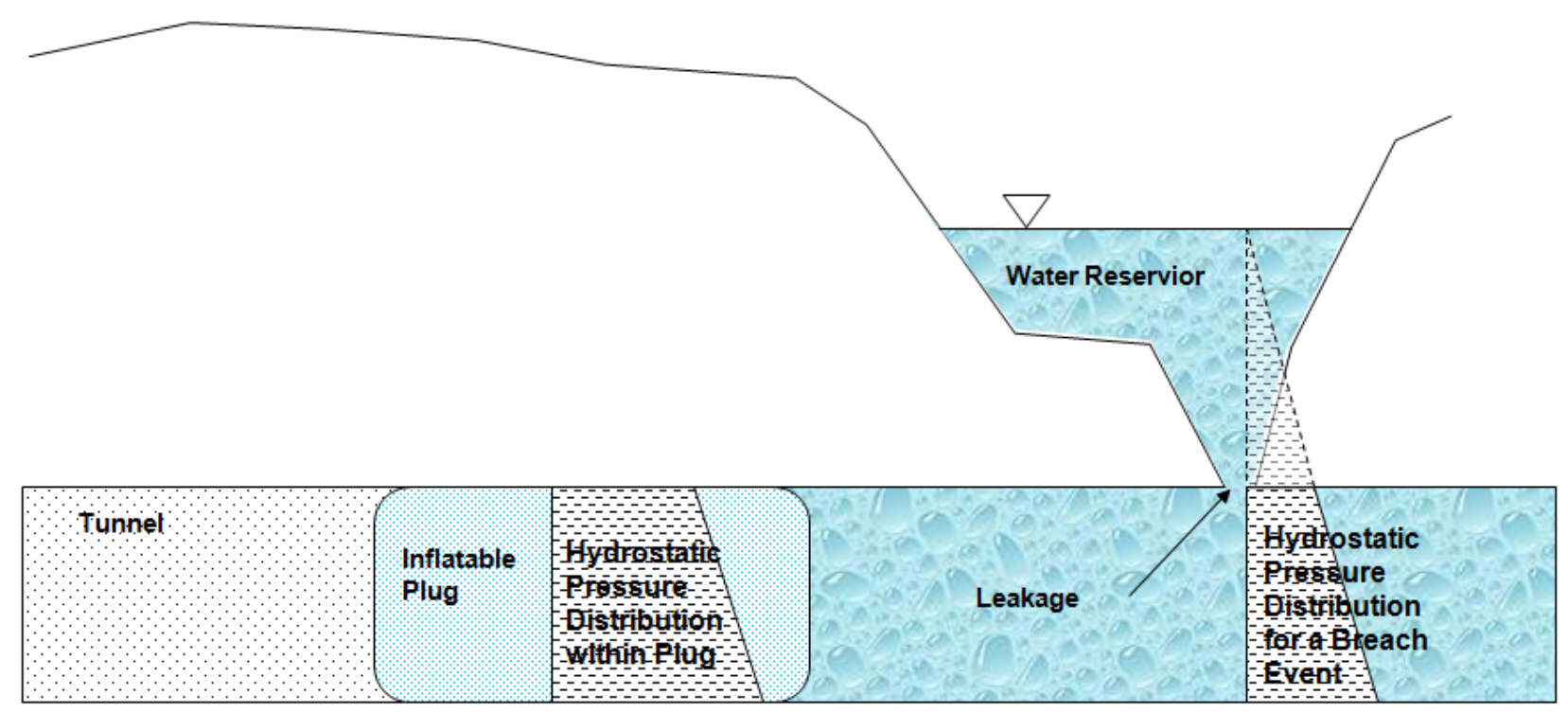

Figure 2.7: Scenario of tunnel leakage that causes flooding in the tunnel.

The equation of hydrostatic pressure is used to calculate the pressure distribution along the Z-axis (Figure 2.8). For an incompressible fluid (such as water) the pressure difference between two elevations can be expressed as:

$p_{2}-p_{1}=-\gamma\left(z_{2}-z_{1}\right)$ 
where $\gamma$ is specific weight of fluid, which is equal to the density of fluid times gravity force, $\rho g$. The pressure at the ceiling of the tunnel, $z_{2}$, is represented as $p_{2} ; p_{1}$ is pressure at the tunnel floor located at the elevation, $z_{1}$.

In order to maintain the axial stability of the system, the average internal or plug pressure $\mathrm{P}_{\mathrm{i}}=\left(\frac{\mathrm{pi1}+\mathrm{pi} 2}{2}\right)$ must be greater or at least equal to the average external pressure, $\mathrm{P}_{\mathrm{e}}=\left(\frac{\mathrm{pe} 1+\mathrm{pe} 2}{2}\right)$.

Chapter

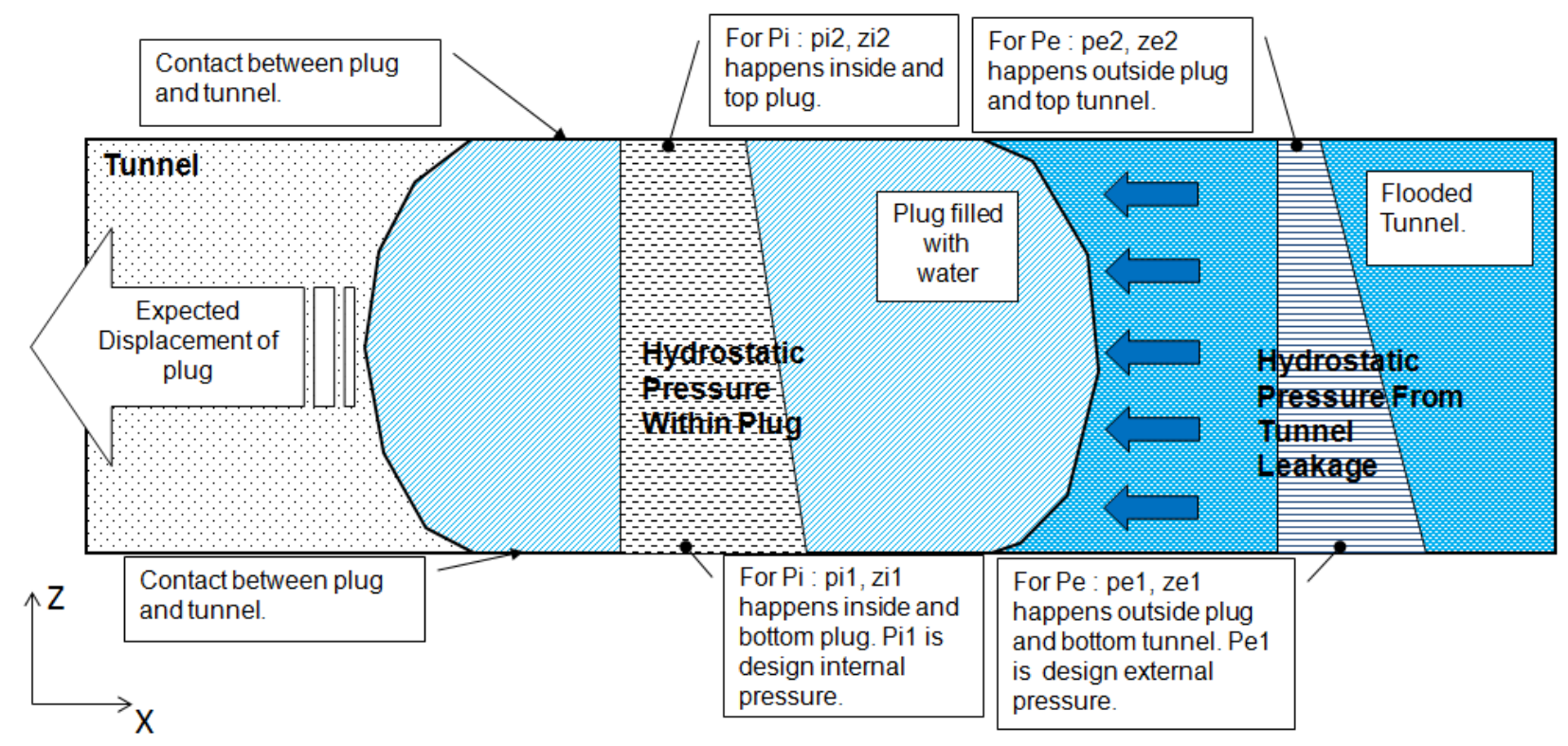

Figure 2.8: Schematic of hydrostatic pressure distributions.

\subsection{Analysis Type}

\subsubsection{Explicit Dynamic Analysis}

Explicit dynamic analysis is usually used for large models with relatively short dynamic response times and for models that have discontinuous events or processes. This type of analysis is used to simulate large rotations and deformations using largedeformation theory [42-43]. 
In this research, explicit dynamic analysis was used to solve the quasi-static problem with complex contact conditions and interactions. The explicit integration rule using diagonal or "lumped" element mass matrices was implemented in the explicit dynamics analysis and explicit central difference integration rule was utilized to define the motion for the body [42-43] as follows,

$\dot{u}^{\left(i+\frac{1}{2}\right)}=\dot{u}^{\left(i-\frac{1}{2}\right)}+\frac{\Delta t^{(i+1)}+\Delta t^{(i)}}{2} \ddot{u}^{(i)}$

$u^{(i+1)}=u^{(i)}+\Delta t^{(i+1)} \dot{u}^{\left(i+\frac{1}{2}\right)}$

where $\dot{u}$ is velocity and $\ddot{u}$ is acceleration. The increment number in an explicit dynamic step was indicated by $i$ and mid-increment values were referred to $(i-1 / 2)$ and $(i+1 / 2)$ [32-33]. Using diagonal element mass matrices is the key to the computational efficiency of the explicit procedure when calculating the accelerations at the beginning of the increment [42-43].

$\ddot{u}^{(i)}=M^{-1} \cdot\left(F^{(i)}-I^{(i)}\right)$

where the acceleration $\ddot{u}^{(i)}$ is obtained from the inversion of mass matrix $M^{-1}$, the applied load vector $F^{(i)}$, and the internal force vector $I^{(i)}$ at increment number $i$. The explicit dynamic procedure does not require iterations and tangent stiffness matrix [4243].

For presentation of results, initial conditions, and certain constraints, the mean velocities, $\dot{u}^{(i \pm 1 / 2)}$, requires special treatment and the state velocities can be obtained through interpolating the mean velocities linearly [42-43].

$\dot{u}^{(i+1)}=\dot{u}^{\left(i+\frac{1}{2}\right)}+\frac{1}{2} \Delta t^{(i+1)} \ddot{u}^{(i+1)}$

The number of mean velocities, $\dot{u}^{(-1 / 2)}$, needs to be input in order to activate the center difference operator, therefore, at time $=0$, the initial magnitudes of the velocity and acceleration are zero unless they are indicated specifically [42-43]. 
$\dot{u}^{\left(+\frac{1}{2}\right)}=\dot{u}^{(0)}+\frac{\Delta t^{(1)}}{2} \ddot{u}^{(0)}$

$\dot{u}^{\left(-\frac{1}{2}\right)}=\dot{u}^{(0)}-\frac{\Delta t^{(0)}}{2} \ddot{u}^{(0)}$

\subsection{Mass Scaling}

When a quasi-static or dynamic model has some very small elements which affect the stable time increment during simulation, mass scaling is one of the analysis techniques that can be used to improve the computational efficiency [42-43]. Since the explicit central difference method (see equation 2.81 and 2.82) is utilized to integrate the equations in time, the discrete mass matrix (see equation 2.83) that used in the equilibrium equation affects both computational efficiency and accuracy [42-43]. However, mass scaling techniques are more appropriate for quasi-static analysis than dynamic analysis since overly mass scaling would affect the dynamic response of the model [42-43]. Therefore, the changes in mass and dynamic consequent should be ensured, so that the inertial forces do not change the solution significantly [42-43].

In general, the mass scaling strategies can be performed by defining a constant factor to the specified elements and/or setting a minimum stable time increment for specified elements [42-43]. Mass scaling strategies [42-43] can executed through

- modifying the densities of the materials in the model in order to offer much more flexibility.

- scaling the mass of the entire model or specific individual elements/element sets

- $\quad$ scaling the mass on every step in a multistep analysis

- $\quad$ scaling the mass at the beginning and/or throughout the step

The representations of the physical mass and inertia in the model are required to capture the transient response in dynamic analysis. Thus, the natural time scale is important. When a dynamic model has small elements, Abaqus/Explicit will be forced to use a small time increment to integrate the whole model [42-43]. The stable time increment will be improved significantly without affecting the overall dynamic behavior of 
the model if scaling the masses of these small elements at the beginning of the simulation [42-43]. The stable time increment equation without considering damping can be written as

$\Delta t \leq \min \left(L^{e l} \sqrt{\frac{\rho}{\lambda+2 \mu}}\right)$

where $L^{e l}$ is the element with the smallest length, $\rho$ is the density of material, and $\lambda$ and $\mu$ are the Lamé's constants of the materials. Abaqus' manuals do not address the mass scaling approaches in term the mass matrix equation, however, they do explain that the cost of simulation can be defined directly to the number of time increments $(n)$ if time increment $(\Delta t)$ remains constant [42-43]. The number of time increment can be expressed as

$n=T \max \left(\frac{1}{L^{e l}} \sqrt{\frac{\lambda+2 \mu}{\rho}}\right)$

where $T$ is the time period of the simulation event $(n \cdot \Delta t)$. The mass scaling approach is using an artificial factor $\left(f^{2}\right)$ to increase the material density $(\rho)$ and decrease the event time $(T)$ [42-43].

In a quasi-static analysis, Abaqus suggests to verify the mass scaled model through ensuring the ratio $\left(\frac{E_{k}}{E_{i}}\right)$ percentage lower than $10 \%$ between kinetic energy, $E_{k}$, and internal energy, $E_{i}$. This will typically ensure the inertia forces do not dominate and alter the simulation result [42-43].

The mass scaling approach will not affect thermal solution response, gravity loads, viscous pressure loads, adiabatic heat calculations, EOS materials, fluid and fluid link elements, surface-based fluid cavities, and spring and dashpot elements [42-43]. Further details on mass scaling implemented in this research is discussed in Chapter 5. 


\subsection{Equations of State (EOS)}

Equations of State (EOS) can be used to simulate a hydrodynamic behavior of material. An equation of state is governed by constitutive equation through pressure which in term of the density and the internal energy [42-43].

An incompressible viscous and inviscid laminar flow which is governed by the Navier-Stokes equation of motion, can be modeled through a linear $U_{s}-U_{p}$ equation of state model [42-43]. The general Navier-Stokes equation can be expressed as below,

$\rho\left(\frac{\partial \mathrm{v}}{\partial \mathrm{t}}+\mathrm{v} \cdot \nabla \mathrm{v}\right)=-\nabla p+\nabla \cdot \mathrm{T}+\mathrm{f}$

where $\rho$ is the fluid density, $\nabla$ represents the delta operator, $v$ is the flow velocity, $p$ is the pressure, $T$ is the deviatoric component of the total stress tensor, and $f$ is the body forces per unit volume which acts on the fluid. The volumetric response is governed by the bulk modulus which acts as a penalty parameter for the incompressible constraint through equations of state [42-43].

Abaqus uses the Newtonian viscous deviatoric model and define the real linear viscosity of the fluid to model the viscous laminar flow of Navier-Poisson law of a Newtonian fluid. On the other hand, nonlinear viscosity models can be used to model non-Newtonian viscous flow. To obtain an accurate solution for this class of problems, approximate initial conditions for fluid velocity and stress need to be defined [42-43].

Abaqus suggests to define a small amount of shear resistance to suppress shear modes to prevent element distortion during simulating an incompressible fluid (such as water) in Abaqus/Explicit. Due to the water flow is assumed as inviscid, the shear modulus or viscosity should be small or result in an overly stiff response [42-43]. Abaqus recommends to choose an elastic shear modulus with several orders of magnitude lower than the bulk modulus to avoid overly stiff response. This is because the forces arising due to the volumetric response should be kept several orders of magnitude above the internal forces arising due to the deviatoric response of the material [42-43].

The energy equation without considering heat conduction can be expressed as 
$\rho \frac{\partial E_{m}}{\partial t}=\left(p-p_{b v}\right) \frac{1}{\rho} \frac{\partial \rho}{\partial t}+\mathbf{S}: \dot{\mathbf{e}}+\rho \dot{Q}$

where $\rho$ is the current density, $E_{m}$ is the internal energy per unit mass, $p$ is the pressure stress, $p_{b v}$ is the pressure stress from bulk viscosity, $\mathbf{S}$ is the deviatoric stress tensor, $\dot{\mathbf{e}}$ is the deviatoric strain rate, and $\dot{Q}$ is the heat rate per unit mass [42-43].

The pressure is defined as a function of current density and internal energy per unit mass to define all the equilibriums states that exist in a material,

$p=f\left(\rho, E_{m}\right)$

Equation 2.86 can be simplified to obtain a $p$ (pressure) versus $V$ (current volume)

relationship or, equivalently, written as an unique $p$ versus $\frac{1}{\rho}$ relationship by eliminating the internal energy. This relationship which is locus of $p-V$ states achievable behind a shock is called the Hugoniot curve [42-45]. Figure 2.9 is a schematic representation of a Hugoniot curve. In Figure 2.9, the $p_{H}$ represents the Hugoniot pressure which is a function of density and can be defined from fitting experimental data [42-43].

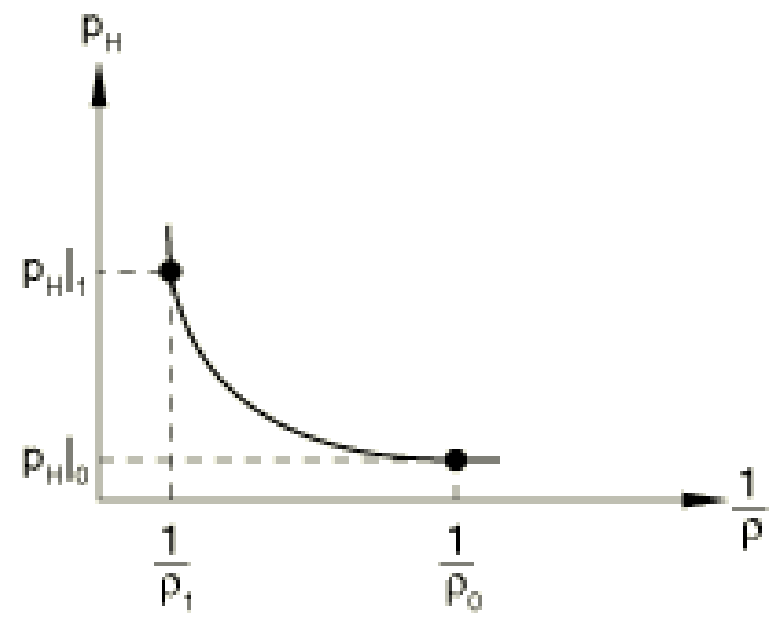

Figure 2.9: A Hugoniot curve [42-43].

When the equation of state is assumed to be linear in energy, the pressure stress can be written as below,

$p=f(\rho)+g(\rho) E_{m}$ 
where $f(\rho)$ and $g(\rho)$ are functions of density only and both functions depend on the particular equation of state model. The Mie-Grüneisen equation of state [42-43, 46-47] is defined as linear in energy and its general form of pressure stress can be expressed as below,

$p-p_{H}=\Gamma \rho\left(E_{m}-E_{H}\right)$

where $p_{H}$ and $E_{H}$ are the Hugoniot pressure and Hugoniot specific energy per unit mass, respectively. Both Hoguniot terms are functions of density only [42-43]. $\Gamma$ is the Grüneisen's gamma defined in term of density ratio,

$\Gamma=\Gamma_{0} \frac{\rho_{0}}{\rho}$

where $\Gamma_{0}$ is the Grüneisen's gamma at the reference state and $\rho_{0}$ is the initial density [42-43]. The Hugoniot specific energy per unit mass can be expressed in term of Hugoniot pressure by

$E_{H}=\frac{p_{H} \eta}{2 \rho_{0}}$

where $\eta$ is the nominal volumetric compressive strain $\left(1-\rho_{0} / \rho\right)$. Equation 2.88 can be rewritten by eliminating the Grüneisen's gamma and Hugoniot specific energy (per unit mass) [42-43],

$p=p_{H}\left(1-\frac{\Gamma_{0} \eta}{2}\right)+\Gamma_{0} \rho_{0} E_{m}$

The coupled equations for pressure and internal energy are represented by the equation of state and the energy equation and Abaqus solves these equations at each material point simultaneously [42-43].

When a general Hugoniot pressure is related to linear shock velocity $\left(U_{s}\right)$ and particle velocity $\left(U_{p}\right)$ by

$p_{H}=\frac{\rho_{0} c_{0}^{2} \eta}{(1-s \eta)^{2}}$ 
where $c_{0}$ is the bulk speed of sound and $s$ is the linear Hugoniot slope coefficient $\left(d U_{s} / d U_{p}\right)$. Furthermore, $\rho_{0} c_{0}^{2}$ is the elastic bulk modulus at small nominal strains [4243]. $c_{0}$ and $s$ define the linear relationship between $U_{s}$ and $U_{p}$ as below,

$U_{s}=c_{0}+s U_{p}$

The linear $U_{s}-U_{p}$ Hugoniot form can be rewritten by inserting the equation 2.91 into equation 2.92,

$p=\frac{\rho_{0} c_{0}^{2} \eta}{(1-s \eta)^{2}}\left(1-\frac{\Gamma_{0} \eta}{2}\right)+\Gamma_{0} \rho_{0} E_{m}$

In Abaqus, the initial state of material needs to be determined by defining the initial internal energy per unit mass $\left(E_{m}\right)$ and pressure stress $(p)$. The initial pressure is deduced from the specific stress states and Abaqus will calculate the initial density $(\rho)$ automatically to satisfy the equation of state (equation 2.86). Abaqus will assume the material has a reference state of zero internal energy, zero pressure stress, and current density equals to initial density [42-43]. Further details of using the $U_{s}-U_{p}$ equation of state to define the water behavior in form of finite element is presented in Chapter 3. 


\section{Reduced-Scale Prototype: Model Evaluation}

\subsection{Introduction}

This chapter presents the finite element (FE) modeling approaches used to determine the material properties and system friction through various parametric studies in a reduced-scale prototype which is one-fourth of a full-scale prototype tested experimentally by Barbero et al. [58].

The modeling techniques, parameters, and material properties obtained in the reduced-scale prototype are later used to verify the local conformance, effective contact length, and induced slippage in a FE model of a full-scale prototype.

This chapter also describes the approach used for creating a simplified model for deployment of the reduced-scale prototype. Most of the simulation results are compared to available experimental data.

\subsection{Structural Membrane}

The structural membrane of the reduced-scale and full-scale plugs is made up of a total of three layers of fabric as shown in Figure 3.1. It was manufactured by ILC Dover and consisted of a bladder, a fabric restraint, and a webbing restraint. The bladder is the inner most layer of the construction and has direct contact interaction with the fluid used for inflation and pressurization. The function of the fabric restraint is to act as a middle layer that protects the bladder. Both bladder and fabric restraint layers are oversized with respect of the webbing restraint to avoid being subjected to membrane stresses generated by the internal pressure. The outer most layer is a macro fabric made up of woven webbings designed to undertake the membrane stresses generated by the pressurization and acts as webbing restraint. The outer layer is the most important structural component while the other two inner layers only contribute to the mass and volume of the plug.

The outer layer is made of Vectran ${ }^{\circledR}$ webbings of $50.8 \mathrm{~mm}(2 \mathrm{in})$ in width. The webbing restraint at end caps requires narrower webbings to attain the correct shape, so $25.4 \mathrm{~mm}$ ( $1 \mathrm{in})$ webbings were used instead. Vectran ${ }^{\circledR}$ is a multifilament yarn spun 
from liquid crystal polymer (LCP). This type of fiber has high strength and modulus, and is able to handle abrasion, impact, and other types of loads as described in [56]. The material properties of each layer of fabric are summarized in Table 3.1.

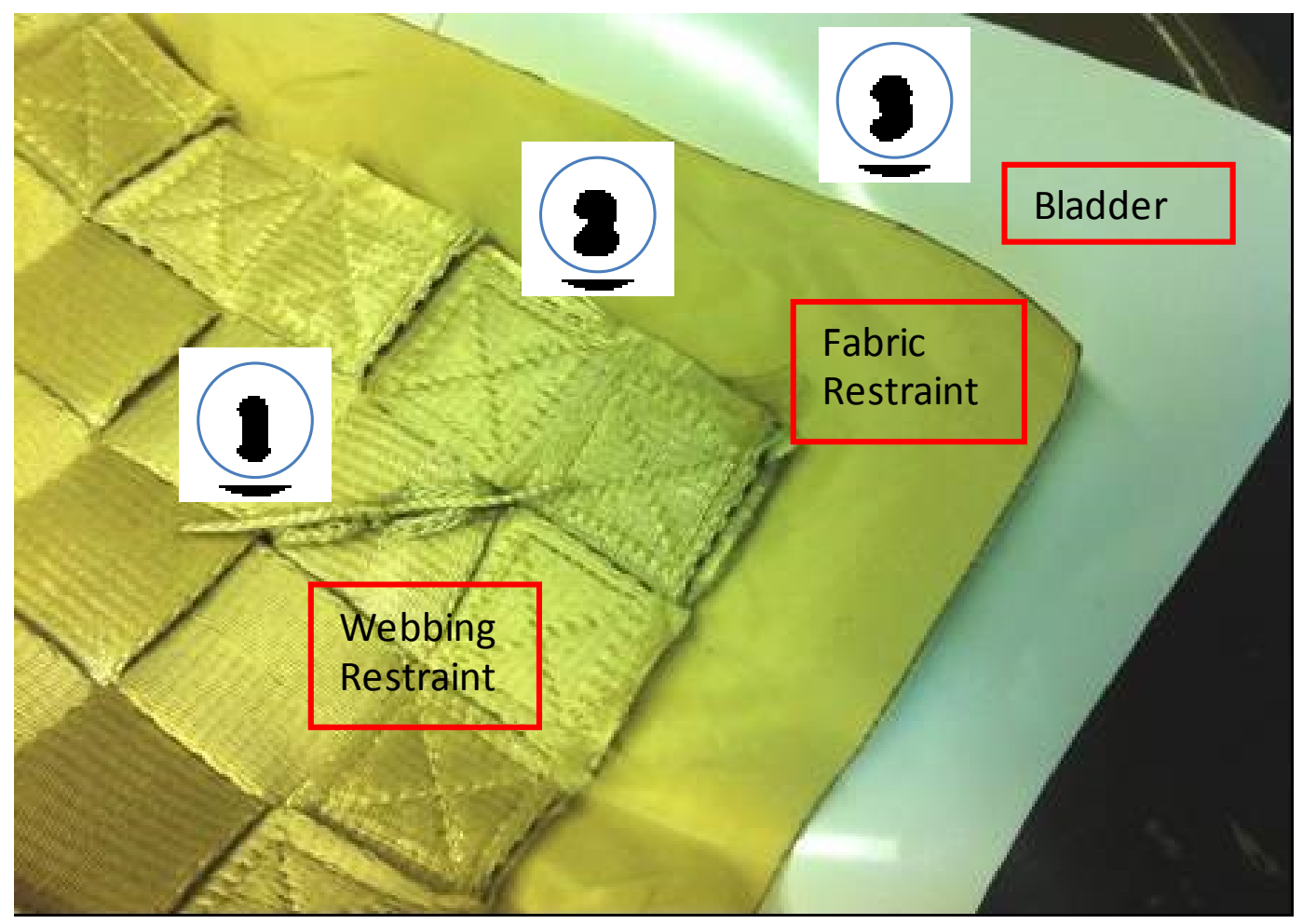

Figure 3.1: Detail of layers of fabric used for the construction of the membrane of reduced and full scale prototypes.

Table 3.1: Material properties of the structural membrane

\begin{tabular}{|c|c|c|c|c|c|c|}
\hline \# & $\begin{array}{l}\text { Layer } \\
\text { Position }\end{array}$ & Component & $\begin{array}{c}\text { Material } \\
\text { Description }\end{array}$ & $\begin{array}{l}\text { Break } \\
\text { Strength } \\
\mathrm{N} / \mathrm{mm}\end{array}$ & $\begin{array}{c}\text { Weight } \\
10^{-4} \\
\mathrm{~g} / \mathrm{mm}^{2}\end{array}$ & $\begin{array}{c}\text { Fabric } \\
\text { Thickness } \\
\mathrm{mm} / 2 \\
\text { layers }\end{array}$ \\
\hline 1 & Outer & $\begin{array}{l}\text { Webbing } \\
\text { Restraint }\end{array}$ & $\begin{array}{c}\text { Vectran } \\
24 \mathrm{~K}, 2.0 " \\
\text { Webbing }\end{array}$ & $\begin{array}{c}2101.5 \\
(590.97 \mathrm{MPa})\end{array}$ & 1.09 & 7.1120 \\
\hline 2 & Middle & $\begin{array}{c}\text { Fabric } \\
\text { Restraint }\end{array}$ & $\begin{array}{c}\text { Vectran } \\
\text { 400denier, } \\
53 \times 53 \text { ypi }\end{array}$ & $\begin{array}{c}140.1014 \\
(6.8998 \mathrm{MPa})\end{array}$ & 2.04 & 0.3175 \\
\hline 3 & Inner & Bladder & $\begin{array}{c}\# 857 \text { 500d } \\
\text { Polyster }\end{array}$ & - & 3.73 & 0.3683 \\
\hline \multicolumn{5}{|c|}{ Total (values used in the FE models) } & 6.85 & $\begin{array}{c}7.7978 \\
(0.307 \mathrm{in})\end{array}$ \\
\hline
\end{tabular}




\subsection{Assumptions for Modeling of Reduced-Scale Prototype}

The FE model of a reduced scale prototype were built based on the following assumptions:

- The tri-layer construction is represented by one equivalent layer built of an equivalent fabric with the same mass and thickness as the three-layer construction.

- The membrane strength of the equivalent single layer is provided only by the strength of the outer layer of three-layer system.

- Ropes in FE model use the same material properties as the equivalent single layer and have equivalent cross sectional area as real ropes installed in actual prototype.

- Shear strength, and self-friction of the Vectran ${ }^{\circledR}$ woven webbing were obtained through experimentation on material at coupon level [60].

- The time step implemented in the simulations is proportional to the real time used in actual experiments.

- The tunnel perimeter and inflation port fittings installed on the plug are assumed to be rigid bodies.

\subsection{Modeling Plan of Reduced-Scale Prototype}

The creation of a FE model of reduced-scale prototype was the starting point for the development of this work. FE models of a reduced-scale prototype were used to calibrate different parameters later used on the FE models of the full-scale prototype. The key parameters that need to be calibrated through comparison between theoretical or experimental and numerical results are the following:

i) The membrane stresses generated by pressurization of the inflatable plug under unconstrained conditions. The material properties that defined the constitutive model implemented in the FE simulations were obtained from uniaxial tests on individual webbings. Since the outer layer is considered to be the only load bearing member of the inflatable structure, the contribution of the other two layers is neglected. Moreover, this layer is treated as an orthotropic material since the structural layer is composed by webbings woven in a plain weave fashion. An FE model of an 
unconstrained plug was created in order to compare the theoretical and numerical hoop $\left(S_{11}\right)$ and longitudinal $\left(S_{22}\right)$ stresses generated by an internal pressure $\left(P_{i}\right)$ applied on the reduced-scale plug. This test would confirm the correspondence between the internal pressure and stresses.

ii) The axial elongation for constrained conditions provided key information to calibrate the stiffness of the model. The availability of experimental results allowed the adjustment of the material properties of the FE model. A series of elongation test were performed to verify the key elements of fabric's material properties that correspond to the Equation 2.41 in terms of tensile stress $\left(T_{11}\right)$, compressive stress $\left(T_{22}\right)$, tensile strain $\left(n_{1}\right)$, and compressive strain $\left(n_{2}\right)$ in both fill (component 1 ) and warp (component 2 ) directions. The values of stresses and strains obtained through simulation of elongation tests were used to adjust the material properties of the equivalent single layer membrane. The elongation tests were performed under constrained conditions and the numerical results compared to experimental results.

iii) The friction coefficient between the inflatable and the tunnel wall is an important property to ensure axial stability of the system. The value obtained experimentally by induced slippage was reproduced numerically and provided a basis for comparison of results obtained with other values that were not tested experimentally. The friction coefficient between the webbing strip and webbing strip of the inflatable was obtained from experimental friction test on woven webbings [60]. Induced slippage tests that involved changes in the inflation pressure $\left(P_{i}\right)$ and external pressure $\left(P_{e}\right)$ were designed to catch a glimpse of the friction coefficient (between the plug and tunnel wall) that would be used in the FE model of the full-scale plug. The induced slippage tests were conducted under constrained conditions and the behavior was measured by the holding resistance defined as the ratio of external pressure and inflation pressure $\left(\frac{P_{e}}{P_{i}}\right)$. Preliminary analysis of the reduced-scale model demonstrated that the difference between uniform and hydrostatic pressure distributions was not significant (around 4\%) and the computational cost was relatively more expensive for models that included a hydrostatic distribution; 
therefore, the uniform pressure distribution was selected for further evaluations in FE models of the reduced-scale prototype.

\subsection{FE Model Set-up of Reduced-Scale Prototype}

\subsubsection{Modeling Tools}

Altair's HyperMesh $\AA$ and Abaqus/CAE were chosen as finite element preprocessors for creating the geometry, meshing and renumbering the nodes and elements into a scripting-friendly form. For solving the models, different Abaqus packages were selected to solve the simulations. In the post-processing phase, Python, Tool Command Language (TCL) Script, and Abaqus Scripting were implemented to generate numerical outputs, and Abaqus/CAE was used to visualize and present the numerical results graphically. The fundamentals of the Finite Element Method (FEM) and the theoretical aspects that support the different features implemented by Abaqus that are relevant to this dissertation were summarized previously in Chapter 2.

\subsubsection{Geometry}

The reduced-scale prototype consists of a cylinder and two hemispherical endcaps. The transition from the cylindrical portion to the end caps is delimitated by ropes. These ropes also delimit zones of different densities of longitudinal webbings, as illustrated in Figure 3.2. The hemispherical end-cap has diameter of $1244.6 \mathrm{~mm}$ (49 inches) with three partitions. The cylinder has a nominal length of $1143.0 \mathrm{~mm}$ ( 45 inches) and a nominal diameter of $1244.6 \mathrm{~mm}$ (49 inches), as shown in Figure 3.2. The ropes are manufactured with the same fiber used for the webbings (Vectran) with a diameter of $25.4 \mathrm{~mm}$ (1.0 inch). Two round fittings made of Aluminum 7075 were assembled onto one of the hemispherical end-caps as air or water filling ports and they both have same diameter of $203.2 \mathrm{~mm}$ (8 inches). Figure 3.2 shows the actual reduced-scale prototype along with sketches illustrating the nominal dimensions used for the construction of the FE model. 


\subsubsection{Element Selection and Meshing}

\subsubsection{Membrane of the Inflatable}

Element type selection has to be very careful because the selected element type has to be able to simulate the characteristics of the actual material used for manufacturing the prototypes. The membrane element only takes in-plane stiffness into account. The plate element provides only out-plane stiffness. The shell element has both in-plane and out-plane stiffness. The outer layer formed by woven webbings does not include a coating that could create a stiffer material. Thus, the membrane element M3D4 was a selected to simulate the structural behavior of the cylindrical and hemispherical portions of the inflatable.

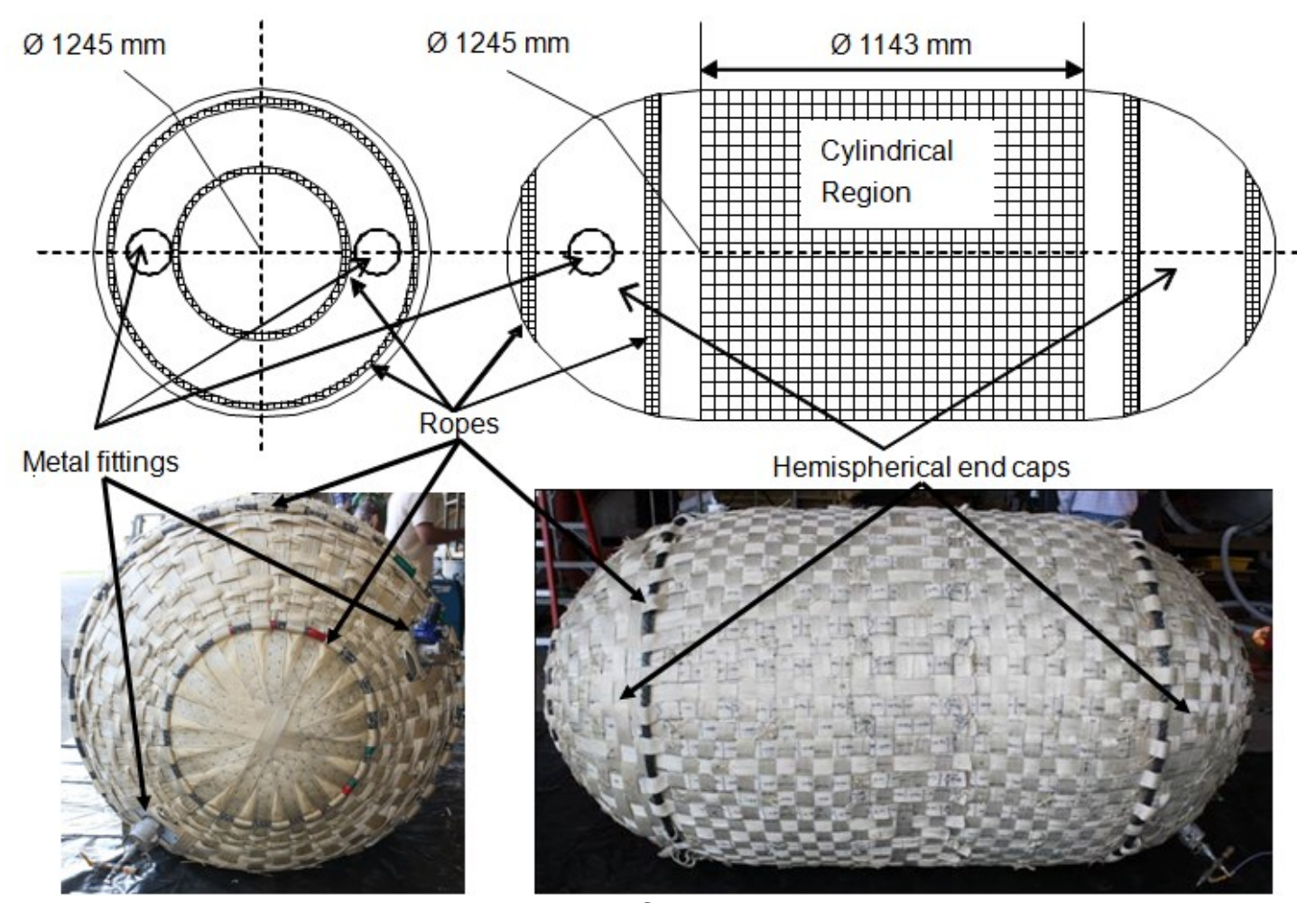

Figure 3.2: Dimensions of reduced scale prototype.

\subsubsection{Tunnel Surface and Inflation Fittings}

The tunnel surface and fittings on the plug are assumed to be very stiff components, when compared to the membrane. Rigid elements of the type R3D4 were 
selected to model the tunnel surface and inflation fittings which are either fixed or undergoing large motions without deformation.

\subsubsection{Mesh Convergence Study}

A preliminary FE model of an unconstrained reduced-scale prototype was created to perform a mesh convergence study. The model of the inflatable was pressurized with $0.4688 \mathrm{MPa}$ (68 psi) and the membrane stresses (hoop and longitudinal) at the cylindrical region were calculated. The convergence was evaluated through 1983, 5592, 10180, and 21191 of elements $(5571,8400,15282$, and 63585 of DOF respectively).

Figure 3.3 and Table 3.2 show that the model with more than 10180 elements (15282 DOF) resulted with an error of $1.86 \%$ and 5 minutes of computational time. An increase in the number of elements to 21121 only reduced the error to $1.53 \%$ and increased the computational time to 10 minutes. Therefore, a model with at least 10000 elements was selected and used for the remaining studies.

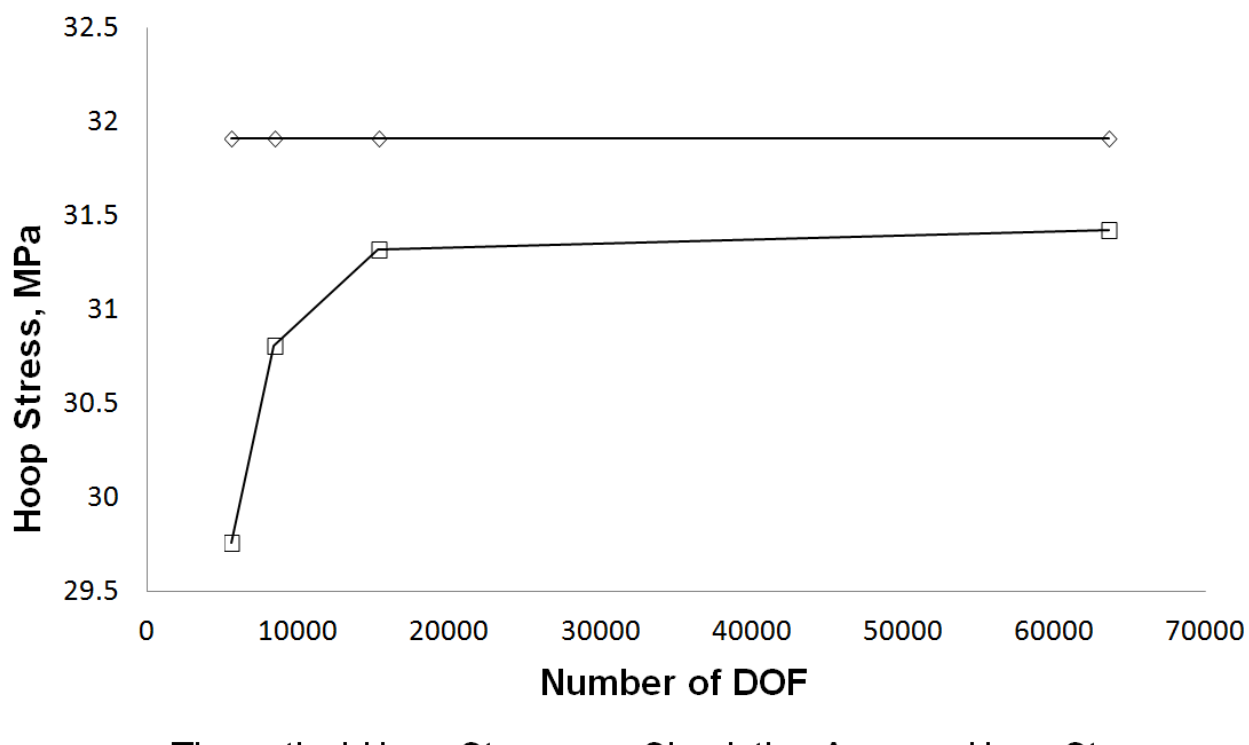

$\because$ Theoretical Hoop Stress $\quad$ Simulation Average Hoop Stress

Figure 3.3: Convergence plot for FE Model of unconstrained reduced-scale prototype. 
Table 3.2: Hoop stress results for unconstrained reduced-scale prototype.

\begin{tabular}{|c|c|c|c|c|c|}
\hline \multirow{2}{*}{$\begin{array}{l}\text { Reduced- } \\
\text { scale } \\
\text { Prototype }\end{array}$} & Number of Elements & 1983 & 5592 & 10180 & 21191 \\
\hline & Number of DOF & 5571 & 8400 & 15282 & 63585 \\
\hline \multirow{5}{*}{$\begin{array}{l}\text { Hoop } \\
\text { Stress } \\
\text { (S11) at } \\
\text { Cylindrical } \\
\text { Region }\end{array}$} & $\begin{array}{l}\text { Theoretical Value, } \\
\mathrm{MPa}\end{array}$ & 31.9135 & 31.9135 & 31.9135 & 31.9135 \\
\hline & $\begin{array}{l}\text { Average Hoop Stress } \\
\text { (S11),MPa }\end{array}$ & 29.7596 & 30.8107 & 31.3211 & 31.4237 \\
\hline & Difference & 2.1539 & 1.1028 & 0.5924 & 0.4898 \\
\hline & Error \% & $6.75 \%$ & $3.46 \%$ & $1.86 \%$ & $1.53 \%$ \\
\hline & $\begin{array}{l}\text { Computational Cost } \\
\text { (mins) }\end{array}$ & 1 & 2 & 5 & 10 \\
\hline
\end{tabular}

\subsection{Modeling of Simplified Deployment of Reduced-Scale Prototype}

This section describes an approach used to create a simplified deployment model of the reduced-scale prototype into the confining surface representative of a tunnel or a large-diameter pipe. The diameter of the plug $(1244.6 \mathrm{~mm})$ is larger than the diameter of tunnel (1219.2 $\mathrm{mm}$ ), so the formation of wrinkles is expected after the plug is completely inflated in the tunnel. The purpose of this approach was to verify the ability of FE model to simulate the initial deflation needed to place the inflatable inside the tunnel. Once the inflatable was positioned, the next step was the simulation of the initial inflation within the confining surface. The advantage of creating a simplified FE model for deployment is the relatively low computational cost. The deployment behavior obtained from this approach cannot be considered as an actual deployment, but it served the purpose of positioning the plug inside the confining surface. Several adjustments were done to adjust the position of the metallic fittings to approximately match their behavior in an actual deployment.

The simplified deployment of the reduced scale prototype is illustrated in Figure 3.4 and the sequence is as follows:

i. Two halves of the tunnel are initially setup to be open at angles of $\pm 45^{\circ}$ and the plug is placed in the middle of the two tunnel halves in inflated position. This initial position is illustrated in Figure 3.4 (1). 
ii. The deployment begins with deflation of the plug by applying vertical gravity force (along Y-axis) while the 2 tunnel halves get closed. The sequence of deflation is shown in Figure 3.4 (2-5).

iii. In Figure 3.4 (6), the deflated plug is left on the tunnel floor for a certain time after the deflation process.

iv. Finally, the plug is then re-inflated by a low magnitude uniform pressure until it is fully inflated and constrained by the tunnel section. The sequence of re-inflation is illustrated in Figure 3.4 (7-10).

1.

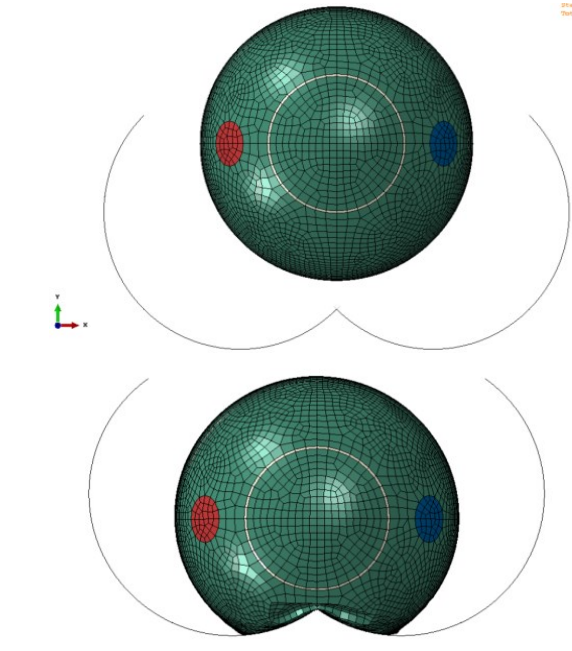

3.

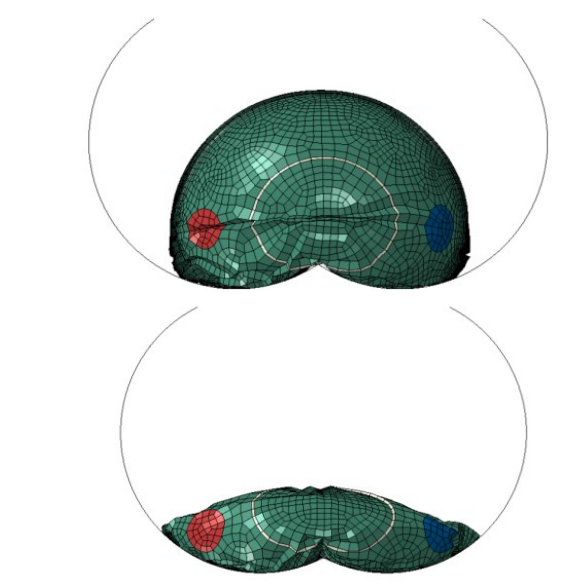

4.

5.

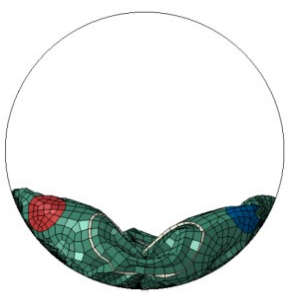

Deflation
6.

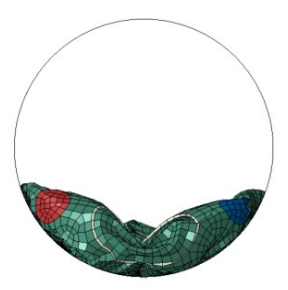

7.

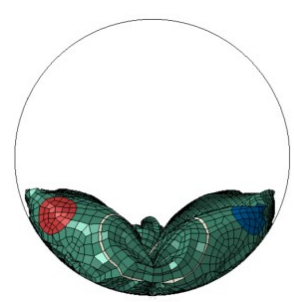

8.

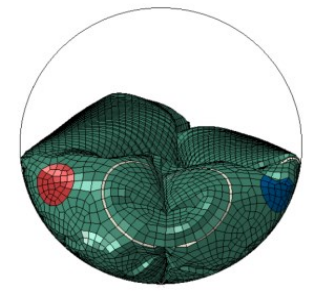

9.

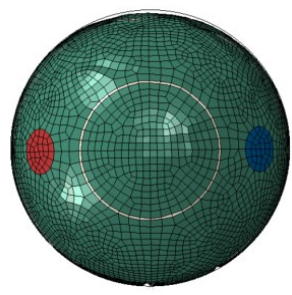

10.

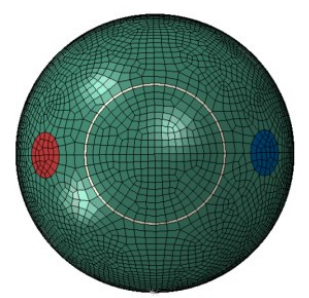

Inflation

Figure 3.4: Sequence of simplified deployment of reduced-scale prototype. 


\subsection{Loading Cases}

\subsubsection{Loading Case I: Stress Evaluation}

Figure 3.5 displays the FE model of reduced-scale prototype under unconstrained pressurization. The reduced scale FE model was meshed with 12972 linear quadrilateral elements of type M3D4 to represent the membrane. Fittings were assumed as rigid bodies and represented by rigid elements of type R3D4.

The longitudinal stress and hoop stress of the reduced scale FE model were compared to the theoretical exact solution in order to validate the fitness of the finite element model. The plug was pressurized up to a maximum of $0.4688 \mathrm{MPa}$ (68 psi), the total thickness of the equivalent fabric is $7.798 \mathrm{~mm}(0.307 \mathrm{in})$ and dimensions of the plug were shown in Figure 3.2.

The inflatable can be considered as a thin-walled pressure vessel composed by two hemispherical ends connected by a cylindrical segment. Theoretically, the hoop stress $\left(S_{11}\right)$ and longitudinal stress $\left(S_{22}\right)$ are expected to be same at hemispherical caps. These stresses are given by,

$S_{11}=S_{22}=\frac{P R}{2 t}$

where $P$ is the pressure applied inside the plug, $R$ is the radius of the hemisphere, and $t$ is the total thickness of fabric. In the cylindrical region, the hoop stress $\left(S_{11}\right)$ and longitudinal stress $\left(S_{22}\right)$ can be obtained by,

$S_{11}=\frac{P R}{t}$

$S_{22}=\frac{P R}{2 t}$

where the $P, R$, and $t$ are internal pressure of plug, radius of cylinder and total thickness of the equivalent fabric, respectively. 


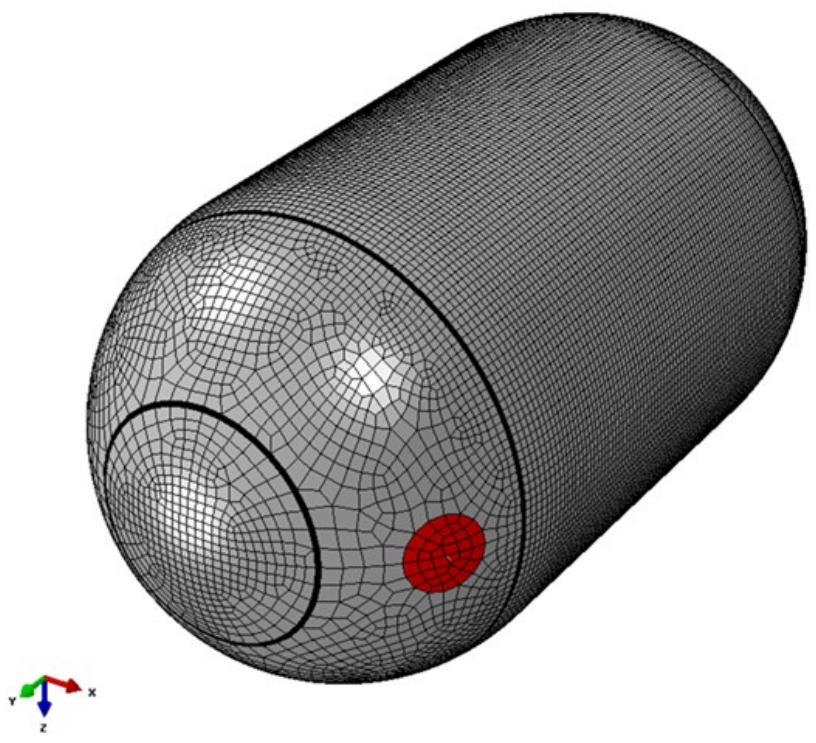

Figure 3.5: Reduced-scale FE model under unconstrained pressurization.

\subsubsection{Loading Case II: Elongation Evaluation}

Since the mechanical properties of the tri-layer system represented by an equivalent single membrane are not readily available, it was necessary to calibrate the stiffness of the model in order to reproduce the experimental results reported in [59]. The calibration was done through axial elongation tests under constrained pressurization.

The elongation under constrained pressurization was measured at the tips of the both hemispherical end caps as schematically shown in Figure 3.6. The experimental elongation data was collected in a stepwise method. The plug was initially pressurized up to $0.1034 \mathrm{MPa}$ (15 psi) using water. This level of pressure remained constant so that the laser meters could measure the total length of the plug. Then, the pressure was increased with increments of $0.1034 \mathrm{MPa}(15 \mathrm{psi})$ until reaching a maximum pressure of $0.4688 \mathrm{MPa}$ (68 psi) as illustrated in Figure 3.7. At each pressure level, the total elongation was measured. The simulation procedure of the reduced-scale plug model was designed similarly to the experimental one but using a proportional step time. The plug was loaded with uniform pressure and carried the mass of water used for pressurization starting from zero to $0.1034 \mathrm{MPa}(15 \mathrm{psi})$ and then increased to 0.2068 MPa (30 psi), $0.3103 \mathrm{MPa}$ (45 psi), $0.4137 \mathrm{MPa}$ (60 psi), and at last $0.4688 \mathrm{MPa}$ (68 
psi). For this simulation, the friction coefficient between the plug and the tunnel walls was assumed to be 0.19 [60] and the shear strength was obtained from experimental data reported in [59]. Figure 3.8 shows the experimental setup used to measure the axial elongation of the reduced scale prototype [60].

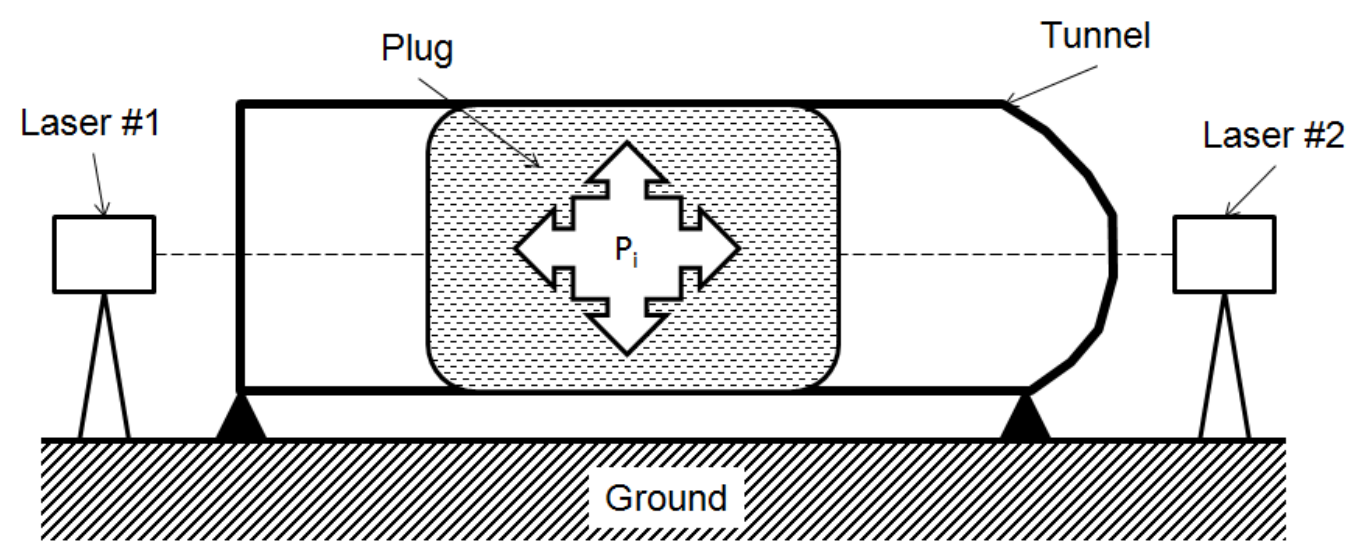

Figure 3.6: Schematic of Constrained Elongation Measurement [59]

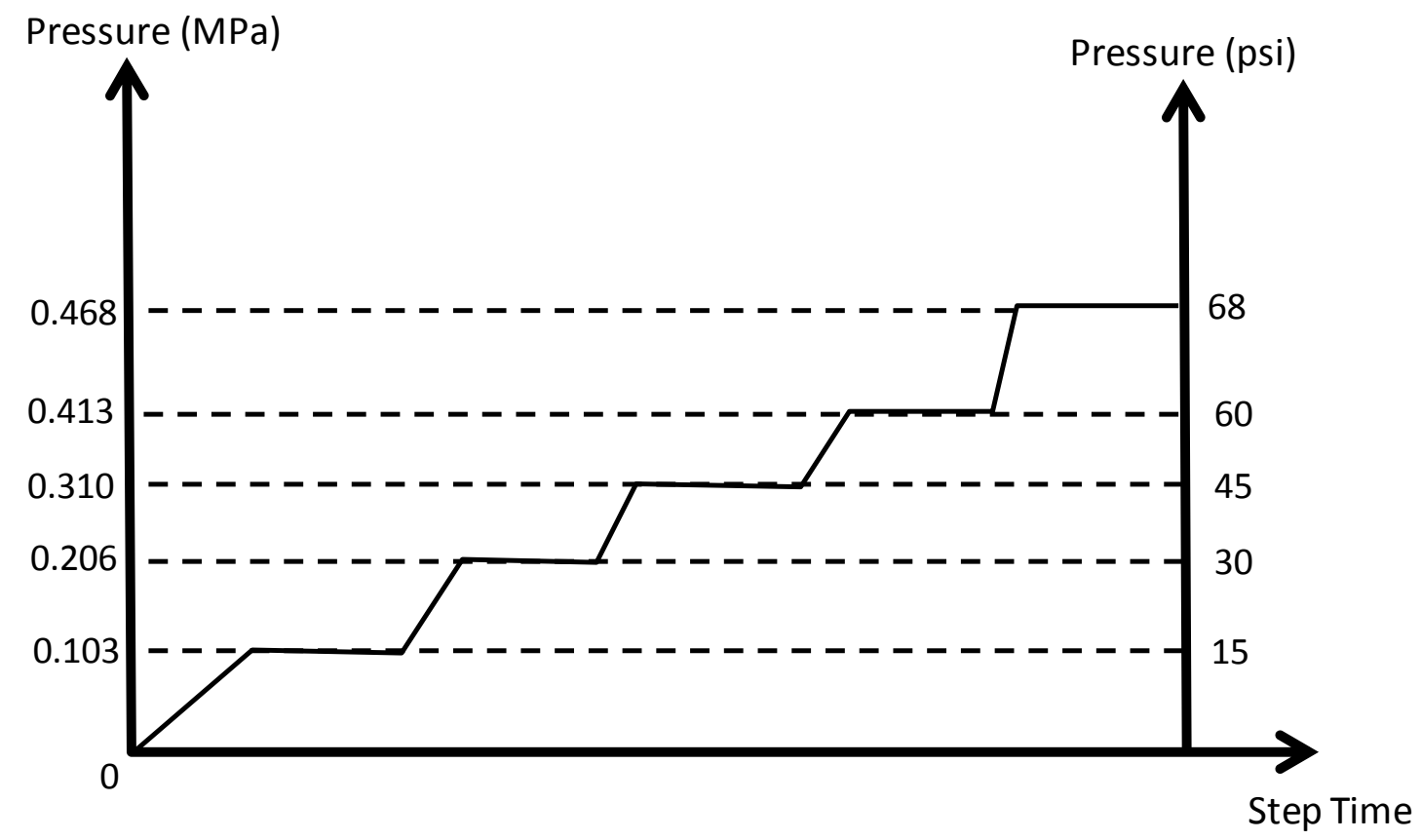

Figure 3.7: Pressurization Procedure for Elongation Test of Constrained Reduced Scale Plug 


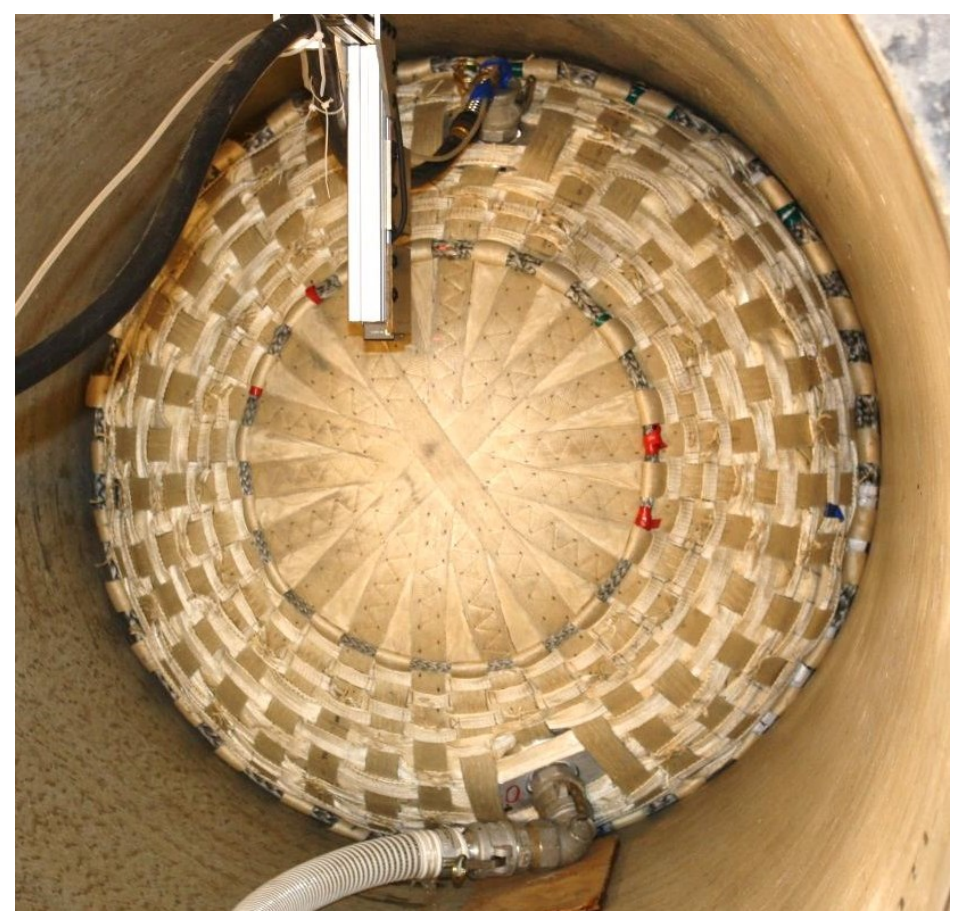

Figure 3.8: Experimental setup for reduced scale prototype tested under constrained pressurization [59].

The maximum strain for all the models was assumed to be 0.1 following the results reported in [60]. A compression stiffness (10\% of tensile stiffness) in the stressstrain curve was added in order to avoid the collapse of wrinkled elements under compressive loading when the plug is deflated. Several candidates for material properties were used to calibrate numerical results with experimental data. Table 3.3 summarizes the different materials considered for calibration. Figure 3.9 shows the diagram of the constitutive model used to represent the material properties summarized in Table 3.3.

The diameter of the rope is $25.4 \mathrm{~mm}$ (1.0 in) and the material properties of the ropes are the same as the orthotropic material properties assigned to membrane elements (tri-layer woven fabric). The equivalent cross sectional area of $506.6441 \mathrm{~mm}^{2}$ $\left(0.7853 \mathrm{in}^{2}\right)$ was added on top of the ropes section to account for their presence in the models. Appendix C summarizes additional details on the determination of the equivalent cross sectional area. The total mass of whole reduced-scale prototype is $56.25 \mathrm{~kg}(124 \mathrm{lb})$. 


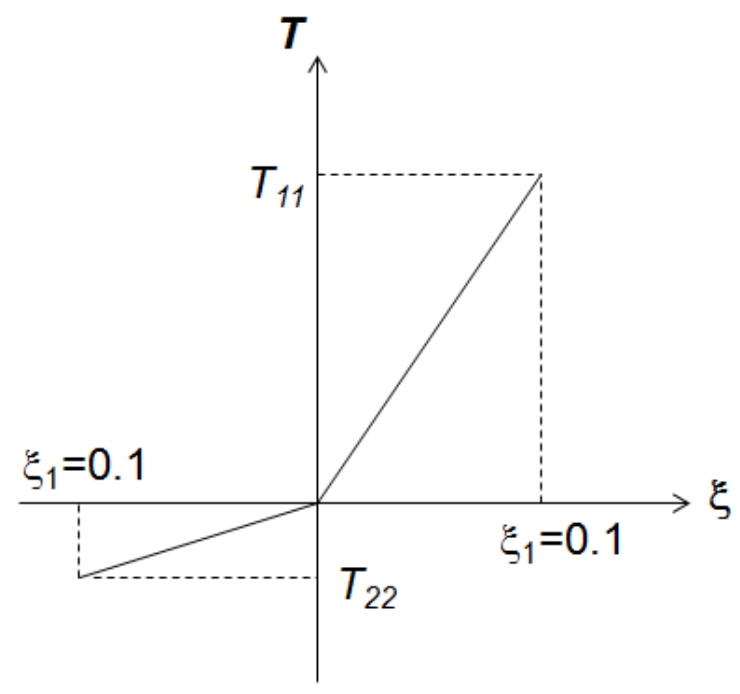

Figure 3.9: Diagram of the constitutive model used in the simulations.

Table 3.3: Candidate of material properties.

\begin{tabular}{|c|c|c|c|c|c|}
\hline Component & $\begin{array}{l}\text { Material Properties } \\
\text { for equivalent fabric }\end{array}$ & $\begin{array}{l}\text { Material } \\
\quad \# 1\end{array}$ & $\begin{array}{l}\text { Material } \\
\quad \# 2\end{array}$ & $\begin{array}{l}\text { Material } \\
\text { \#3 }\end{array}$ & $\begin{array}{l}\text { Material } \\
\quad \# 4\end{array}$ \\
\hline \multirow{2}{*}{$\begin{array}{c}1 \\
\text { (fill yarn } \\
\text { direction) }\end{array}$} & $\begin{array}{c}\text { Tensile Strength, } \\
T_{11}(\mathrm{MPa})\end{array}$ & 590.9772 & 295.4886 & 241.3165 & 258.5534 \\
\hline & $\begin{array}{c}\text { Compressive } \\
\text { Strength, } T_{22}(\mathrm{MPa})\end{array}$ & 59.0977 & 29.5489 & 24.1317 & 25.8553 \\
\hline \multirow{2}{*}{$\begin{array}{c}2 \\
\text { (warp yarn } \\
\text { direction) }\end{array}$} & $\begin{array}{c}\text { Tensile Strength, } \\
T_{11}(\mathrm{MPa})\end{array}$ & 590.9772 & 295.4886 & 241.3165 & 258.5534 \\
\hline & $\begin{array}{c}\text { Compressive } \\
\text { Strength, } T_{22}(\mathrm{MPa})\end{array}$ & 59.0977 & 29.5489 & 24.1317 & 25.8553 \\
\hline
\end{tabular}

\subsubsection{Loading Case III: Axial Slippage Evaluation}

As described in Chapter 1, the purpose of using inflatable structures in confined environments, such as tunnels or large-diameter pipes, is to contain the propagation of hazardous events. One of the most demanding scenarios is the containment of flooding in which the longitudinal axial stability of the inflatable has to be guaranteed. Therefore, the purpose of performing the induced slippage simulations is to predict the stability of the system and to determine the minimum required friction coefficient between the plug and tunnel wall to assure a successful performance. 
Figure 3.10 a shows schematically the main forces involved in a typical axial slippage model including inflation pressure $\left(P_{i}\right)$, external pressure $\left(P_{e}\right)$, mass of fluid within plug, friction $(\mu)$ between the plug and the tunnel wall, and slippage displacement $(U)$. The inflation pressure $\left(P_{i}\right)$ is the pressure that provides the normal component that along with the friction coefficient generates the friction force that resists the effect of the external pressure $\left(P_{e}\right)$ in order to keep the stability of the plug during the containment of the hazardous event. The friction coefficient between the plug and the tunnel was taken from the experimental results reported in [59]. The mass of fluid within plug depends on the fluid (water in this case) that is used to increase the slippage resistance of model.

Figure $3.10 \mathrm{~b}$ presents a longitudinal cut-view of quarter-scale FE model setup for the slippage simulation. The solid element type of C3D8R (linear hexahedral element) is used to mesh the solid geometry that represents water and the water geometry has 3780 and 4468 of total number of elements and nodes, respectively. The main purpose of having the solid elements of water is to evaluate the role of the mass of water in the slippage simulations, especially in the full-scale models. However, the hydrodynamic of water within the plugs is not considered to be a key factor in this study since the water within the plug is assumed to be filled gradually and the external pressure on the rear plug is applied gradually too, therefore, without creating any sloshing or impact scenario. With these considerations, the models developed in this research were setup to reproduce a quasi-static loading scenario in both quarter and full scales.

The linear $U_{s}-U_{p}$ equation of state and Newtonian shear viscosity which were presented in Chapter 2 are recommended by the literature [42-43, 48-51] to define the water behavior (bulk response) in explicit analysis. The volume of solid elements is $98 \%$ $\left(2.3284 \mathrm{~m}^{3}\right)$ of the internal volume of an inflated quarter scale plug. Besides the mass of the plug, the solid elements representing water also added a mass of $2328.4 \mathrm{~kg}$ within plug during the slippage analysis (Figure 3.10a). The gravity force is applied to the solid elements of water from the beginning throughout the entire simulation. The $U_{s}-U_{p}$ equation of state parameters (section 2.10) are taken from Cannmo and Snygg [42-43], and Abaqus benchmark examples [48-51] where the wave speed of water is 45.85 $\mathrm{m} / \mathrm{sec}(1805 \mathrm{in} / \mathrm{sec})$, the density of water is $983.204 \mathrm{~kg} / \mathrm{m}^{3}\left(0.92 \mathrm{E}-4 \mathrm{lb} \mathrm{sec} / \mathrm{in}^{4}\right)$, bulk 
modulus is $2.07 \mathrm{GPa}$ (3.0E5 psi), and shear viscosity of water is $13 \mathrm{E}-4 \mathrm{~Pa} \sec (1.5 \mathrm{E}-8$ psi sec). All these parameters have been verified by comparing with the numerical and experimental solutions that provided by Nakayama et al. [48] and Higuchi et al. [52], respectively.

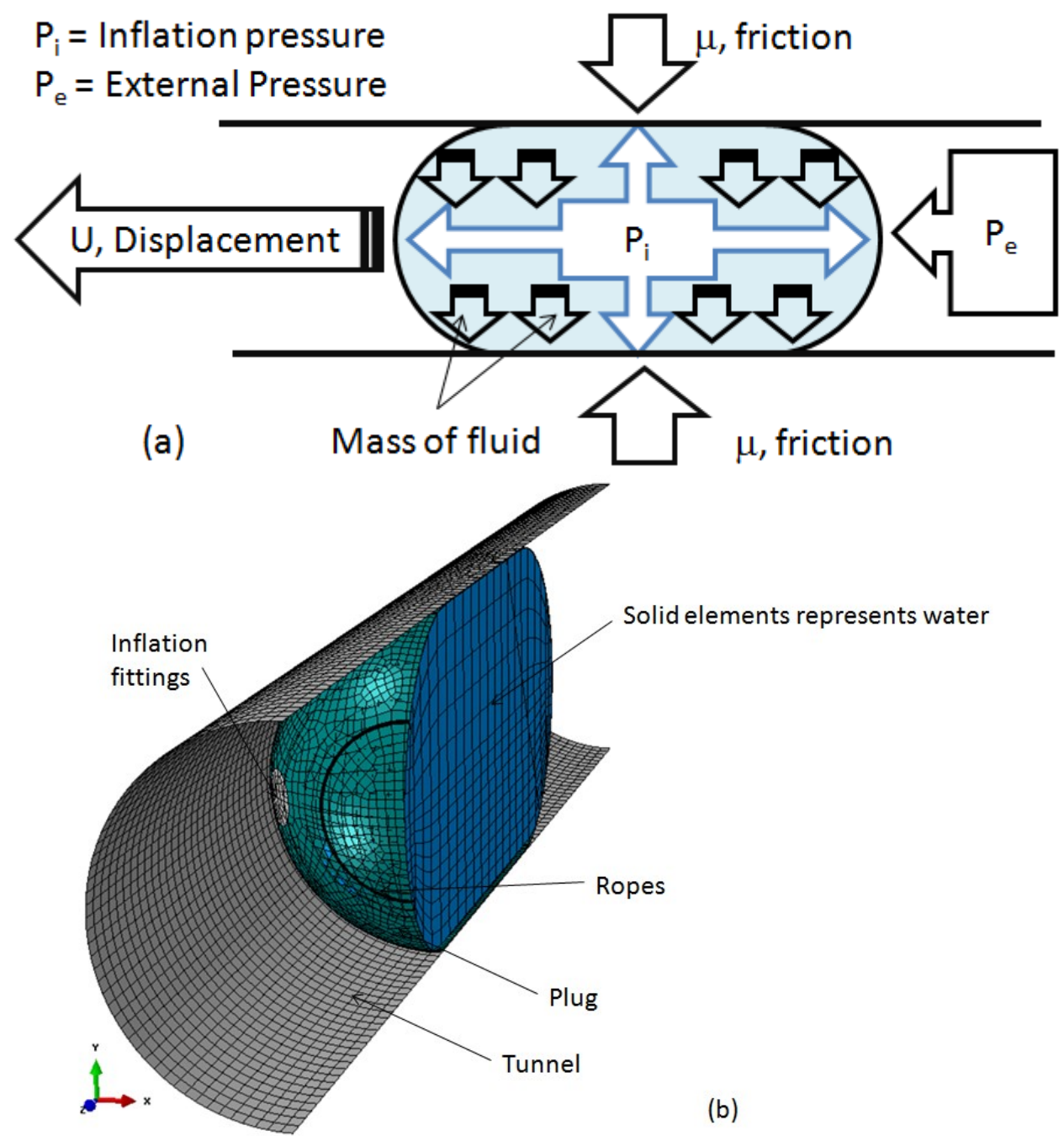

Figure 3.10: (a) Schematic of Axial Slippage Model; (b) Longitudinal Cut-view of FE model setup for slippage simulation

Two pressurization scenarios that can induce longitudinal axial slippage were considered:

a) The first scenario illustrated in Figure 3.11a assumes the presence of a leak in the membrane of the plug that produces gradual depressurization of the plug; or similarly, the pressurization system, typically consisting of pumps or compressors, 
fails to maintain the designated pressure within the plug. The slippage is expected to occur when the inflation pressure $\left(\mathrm{P}_{\mathrm{i}}\right)$ decreases to a level that approaches the magnitude of the external pressure $\left(P_{e}\right)$.

b) The second scenario illustrated in Figure $3.11 \mathrm{~b}$ assumes that the inflated plug withstands an unexpected rise in external pressure $\left(P_{e}\right)$ that approaches to the level of the inflation pressure $\left(P_{i}\right)$ while the inflation pressure $\left(P_{i}\right)$ remains constant during the event.
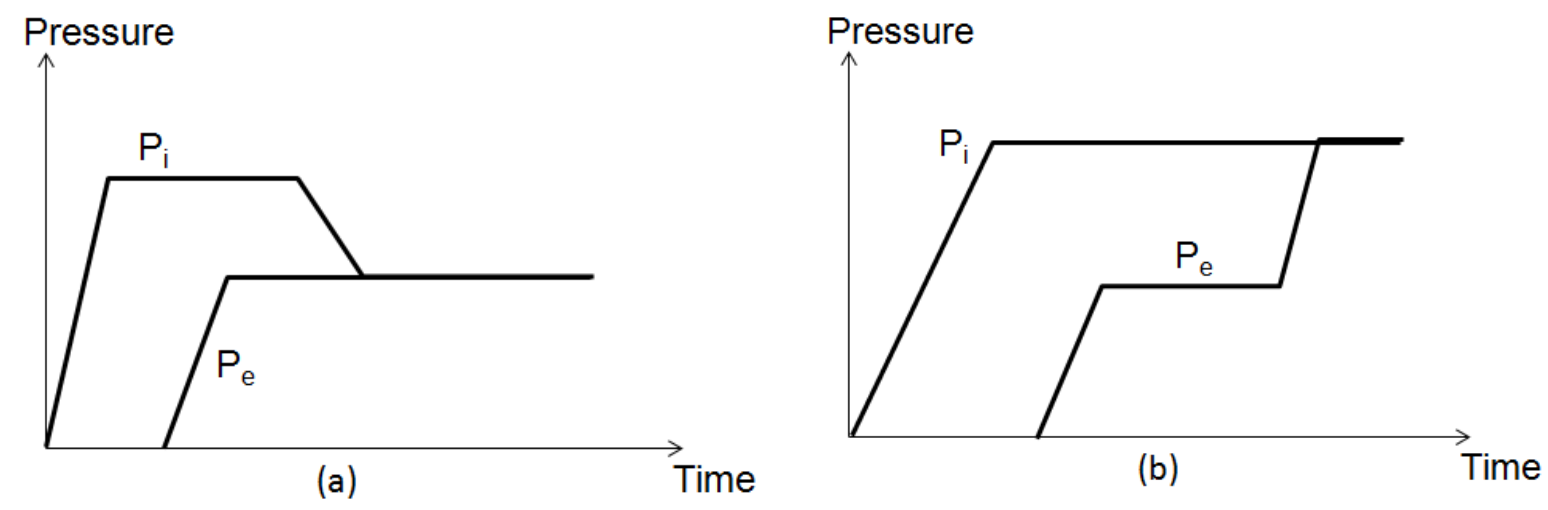

Figure 3.11: (a) Scenario of decreasing inflation pressure $\left(P_{i}\right)$. (b) Scenario of sudden increasing external pressure $\left(P_{e}\right)$.

Scenario (a) of Figure 3.11a is the selected scenario to be simulated due to its relatively simple and low-cost computational implementation as well as the availability of experimental data to compare with [59]. Figure 3.12 shows the detailed pressurization sequence of selected Scenario (a). A uniform pressure, $\mathrm{P}_{\mathrm{i}}$, is applied to the inner surface of the plug starting at the zero second from zero pressure to a specific maximum at the $1^{\text {st }}$ second. The external uniform pressure, $P_{e}$, is assumed to be applied gradually on the rear end-cap when the plug pressure is constant and at its selected value. From the $8^{\text {th }}$ to $12^{\text {th }}$ second, both the plug and tunnel pressure are constant and at their selected values. Then, the depressurization of the plug starts at the $12^{\text {th }}$ second in which the internal pressure begins to decline at certain depressurization rate $(\mathrm{lb} / \mathrm{sec})$ until it matches the external pressure at $32^{\text {nd }}$ second. 
When $\mathrm{P}_{\mathrm{i}}$ and $\mathrm{P}_{\mathrm{e}}$ are close to each other and depending on the friction coefficient between the plug and tunnel, the slippage is expected to occur. Table 3.4 shows the combinations of maximum internal pressure $\left(P_{i}\right)$ and maximum external pressure $\left(P_{e}\right)$ used in the pressurization procedure. The horizontal axial displacement of a node located at the apex of the plug was used to evaluate the occurrence of slippage for all the pressure combinations.

Table 3.4: Load combinations of maximum internal and external pressure.

\begin{tabular}{|c|c|c|}
\hline Test ID & $\begin{array}{c}\text { Maximum Internal } \\
\text { Pressure, } \mathrm{Pi}\end{array}$ & $\begin{array}{c}\text { Maximum External } \\
\text { Pressure, Pe }\end{array}$ \\
\hline $55 / 40$ & $0.3792 \mathrm{MPa}(55 \mathrm{psi})$ & $0.2758 \mathrm{MPa}(40 \mathrm{psi})$ \\
\hline $40 / 30$ & $0.2758 \mathrm{MPa}(40 \mathrm{psi})$ & $0.2068 \mathrm{MPa}(30 \mathrm{psi})$ \\
\hline $30 / 20$ & $0.2068 \mathrm{MPa}(30 \mathrm{psi})$ & $0.1379 \mathrm{MPa}(20 \mathrm{psi})$ \\
\hline $20 / 10$ & $0.1379 \mathrm{MPa}(20 \mathrm{psi})$ & $0.06895 \mathrm{MPa}(10 \mathrm{psi})$ \\
\hline
\end{tabular}

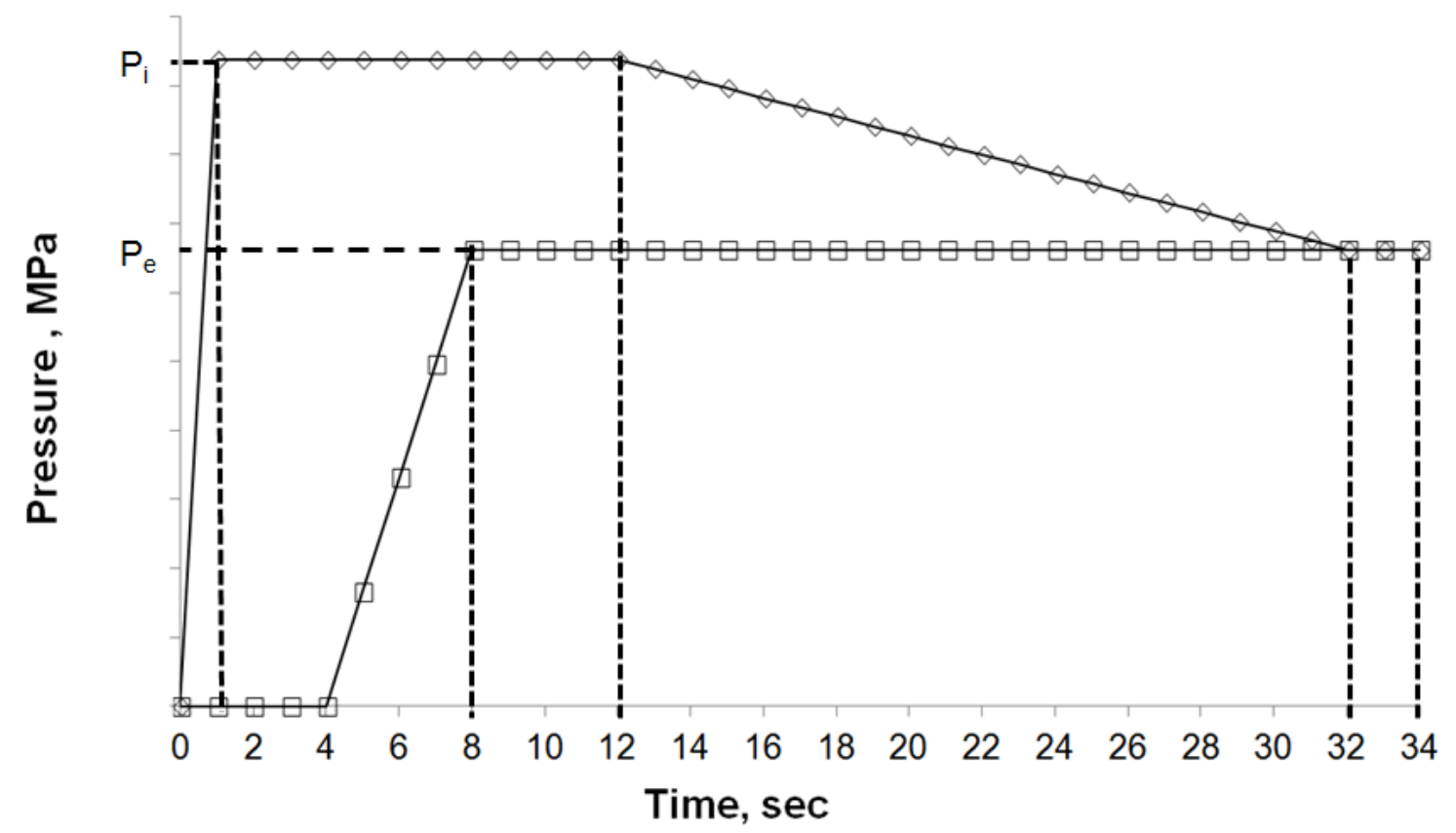

Figure 3.12: Selected Pressurization Sequence for Induced Slippage of Constrained Reduced scale Plug.

The simulations included a solid element in contact with the internal surface of plug to represent the water filling within the plug during the entire pressurization 
process. The properties of water used as input for the simulations are shown in Table 3.5.

Table 3.5: Physical properties of water for simulations.

\begin{tabular}{|c|c|c|}
\hline \multirow{2}{*}{ Material Propeties of Fluid } & \multicolumn{2}{|c|}{ Water at $277.15^{\circ} \mathrm{K}\left(4^{\circ} \mathrm{C}\right)$} \\
\hline \multirow{2}{*}{ Fluid Density } & $\mathrm{kg} / \mathrm{m}^{3}$ & 1000 \\
\cline { 2 - 3 } & $\mathrm{lbm} / \mathrm{in}^{3}$ & $3.612 \mathrm{E}-2$ \\
\cline { 2 - 3 } & $\mathrm{lbf} \cdot \mathrm{sec}^{2} / \mathrm{in}^{4}$ & $9.4 \mathrm{E}-5$ \\
\hline \multirow{2}{*}{ Fluid Bulk Modulus } & $\mathrm{MPa}$ & $2.15 \mathrm{E} 3$ \\
\cline { 2 - 3 } & $\mathrm{lbf} / \mathrm{in}^{2}$ & $3.12 \mathrm{E} 5$ \\
\hline Fluid Expansion Coefficient & $/ \mathrm{K}$ & $2.07 \mathrm{E}-4$ \\
\hline
\end{tabular}

\subsection{Results of Loading Cases \\ 3.8.1 Loading Case I: Stress Evaluation}

A contour of hoop stress and longitudinal stress obtained with the FE model is illustrated in Figure 3.13. At the hemispherical cap region, the theoretical hoop and longitudinal stresses are $15.96 \mathrm{MPa}$ (2314 psi) based on Equation 3.1. The simulation result shows that the average stress is $16.02 \mathrm{MPa}$ (2323.74 psi) which has $0.41 \%$ error than the theoretical value.

From Figure 3.13, the hoop stress and longitudinal stress at the cylindrical region were distributed differently. According to Equation 3.2, the hoop stress is $31.91 \mathrm{MPa}$ (4628.66 psi) and the simulation result yielded a hoop stress in average of $32.31 \mathrm{MPa}$ (4686.11 psi) which has a difference by $1.24 \%$ error. On the other hand, the longitudinal stress $\left(S_{22}\right)$ in the cylindrical region has same magnitude as the longitudinal or hoop stress in the hemispherical cap regions which is $15.96 \mathrm{MPa}$ (2314.33 psi) and 16.21 MPa (2351.27 psi), corresponding to theoretical and numerical results, respectively. The error in this case is $1.60 \%$. Table 3.6 shows a summary of the stress evaluation and Appendix A shows detailed calculations of both theoretical longitudinal and hoop stresses. 
Table 3.6: Stress evaluation of reduced scale prototype for unconstrained pressurization.

\begin{tabular}{|c|c|c|c|c|c|c|}
\hline \multirow{3}{*}{ Model } & \multicolumn{2}{|c|}{$\begin{array}{c}\text { Front Spherical End } \\
\text { Caps }\end{array}$} & \multicolumn{2}{|c|}{$\begin{array}{c}\text { Rear Spherical End } \\
\text { Caps }\end{array}$} & \multicolumn{2}{|c|}{$\begin{array}{l}\text { Cylindrical } \\
\text { Region }\end{array}$} \\
\hline & \multicolumn{2}{|c|}{$\mathrm{MPa}$} & \multicolumn{2}{|c|}{$\mathrm{MPa}$} & \multicolumn{2}{|c|}{$\mathrm{MPa}$} \\
\hline & $S_{11}$ & $S_{22}$ & $S_{11}$ & $S_{22}$ & $S_{11}$ & $S_{22}$ \\
\hline Theoretical Solution & 15.96 & 15.96 & 15.96 & 15.96 & 31.91 & 15.96 \\
\hline FE Result & 16.03 & 16.12 & 15.96 & 15.98 & 32.31 & 16.21 \\
\hline Difference & 0.08 & .016 & 0.00 & 0.02 & 0.40 & .25 \\
\hline Error \% & \multicolumn{4}{|c|}{$0.41 \%$} & $1.24 \%$ & $1.60 \%$ \\
\hline
\end{tabular}
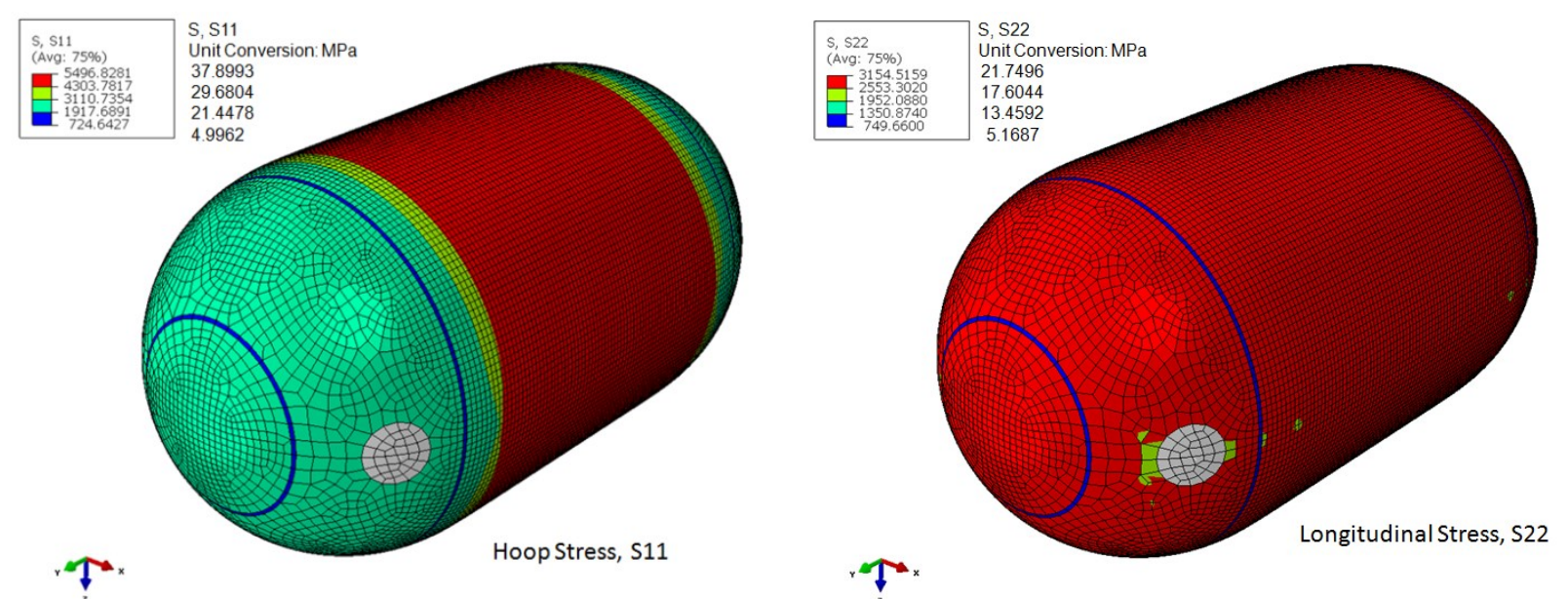

Figure 3.13: Contour of Hoop and longitudinal stresses obtained from FE results for unconstrained pressurization.

\subsubsection{Loading Case II: Elongation Evaluation}

Figure 3.14 shows the FE model for the elongation test under constrained pressurization and Figure 3.15 shows the elongation results obtained from the implementation of candidate materials summarized in Table 3.3. The experimental results corresponding to the tri-layer plug were adjusted with a linear fitting of 
experimental data [59] that was used for comparison with the results obtained from the equivalent single-layer membrane implemented in the FE models. The comparison was made in terms of the proximity of the slope of the fitting lines obtained with the FE models, with respect to the slope of the fitting line obtained from measurements in the actual three layer plug [59].
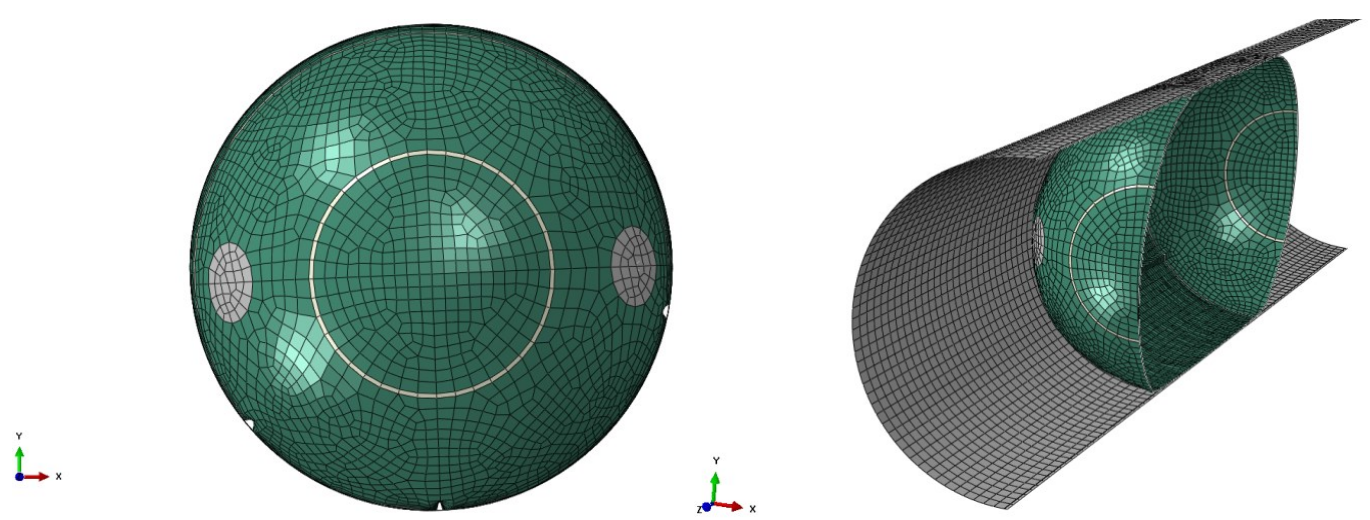

Figure 3.14: FE model performs the elongation test under constrained condition.

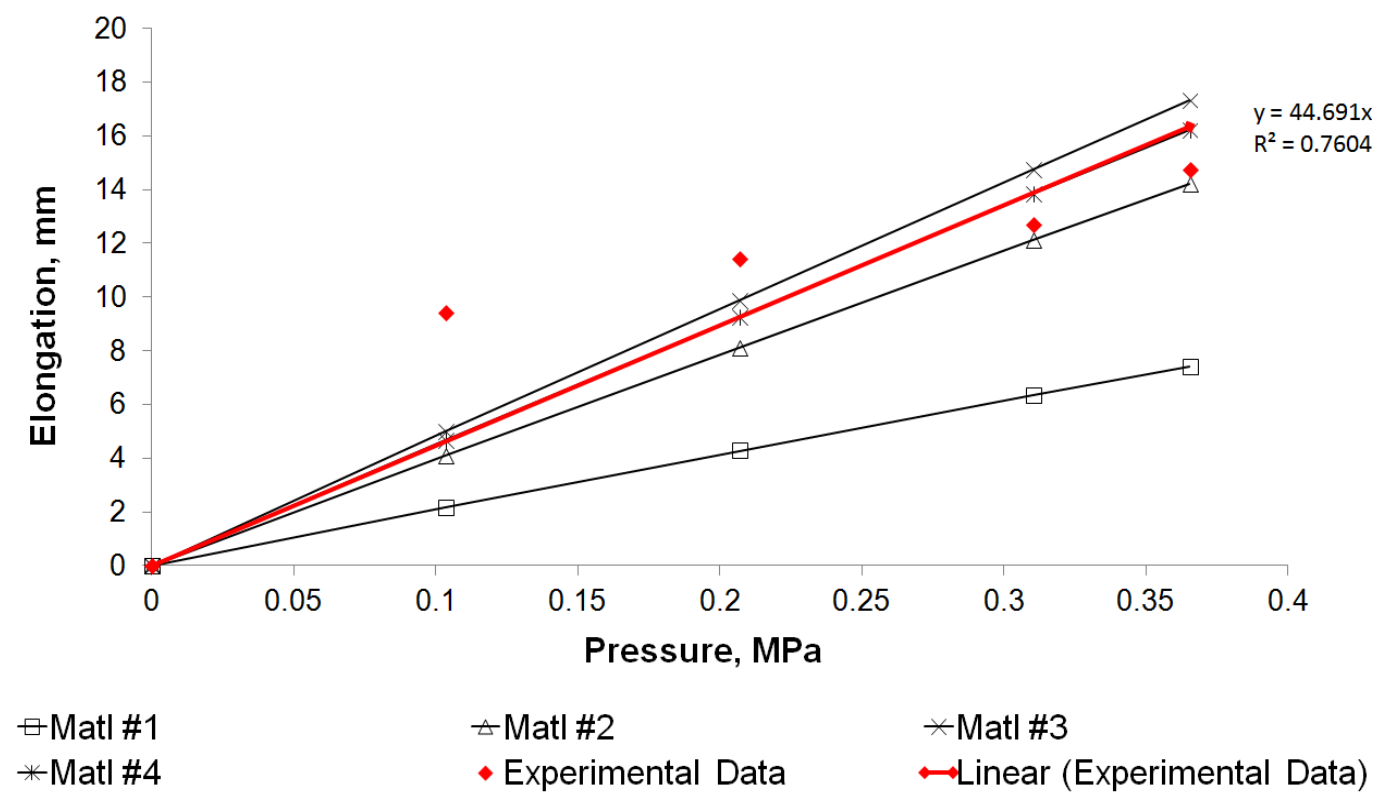

Figure 3.15: Plot of material properties evaluation.

When comparing the elongation results obtained from the material candidates with the slope of the linear fitting of the tri-layer woven fabric, Material \#1 with tensile strength of $590.98 \mathrm{MPa}$ has error of $54 \%$, Material \#2 with tensile strength of 295.49 $\mathrm{MPa}$ has error of $12 \%$, Material \#3 with tensile strength of $241.32 \mathrm{MPa}$ has error of $-7 \%$, 
and Material \#4 with tensile strength of $258.55 \mathrm{MPa}$ has approximately $0 \%$ error. Figure 3.16 shows the variation of error corresponding to each material candidate and the experimental results. Material \#4 has the least error among the candidates. The material selection for the remainder of this work was made based on the minimum error which corresponds to Material \#4.

The stress evaluation under constrained pressurization was done with a friction coefficient between the plug and the tunnel wall of 0.19 and for an inflation pressure of $0.4688 \mathrm{MPa}$ (68 psi). At this pressure, the maximum average stresses (hoop and longitudinal) at the hemispherical end-cap resulted in values of $18.2063 \mathrm{MPa}$ and 19.4386 MPa, respectively. On the other hand, the maximum average stresses (hoop and longitudinal) in the cylindrical region were $8.7068 \mathrm{MPa}$ and $4.7548 \mathrm{MPa}$, respectively, (Table 3.6). The stresses at the hemispherical end cap are nearly $21 \%$ higher than the values for unconstrained pressurization (see Table 3.6); while the hoop and longitudinal stresses at the cylindrical region are $335 \%$ and $366 \%$, respectively, lower than the values corresponding to unconfined pressurization. These results indicate that the confining effect of the tunnel is relieving considerably the hoop and longitudinal stresses in the cylindrical portion while slightly increasing the stresses in the hemi-spherical end-caps. The stress distribution for confined pressurization is illustrated in Figure 3.17. 


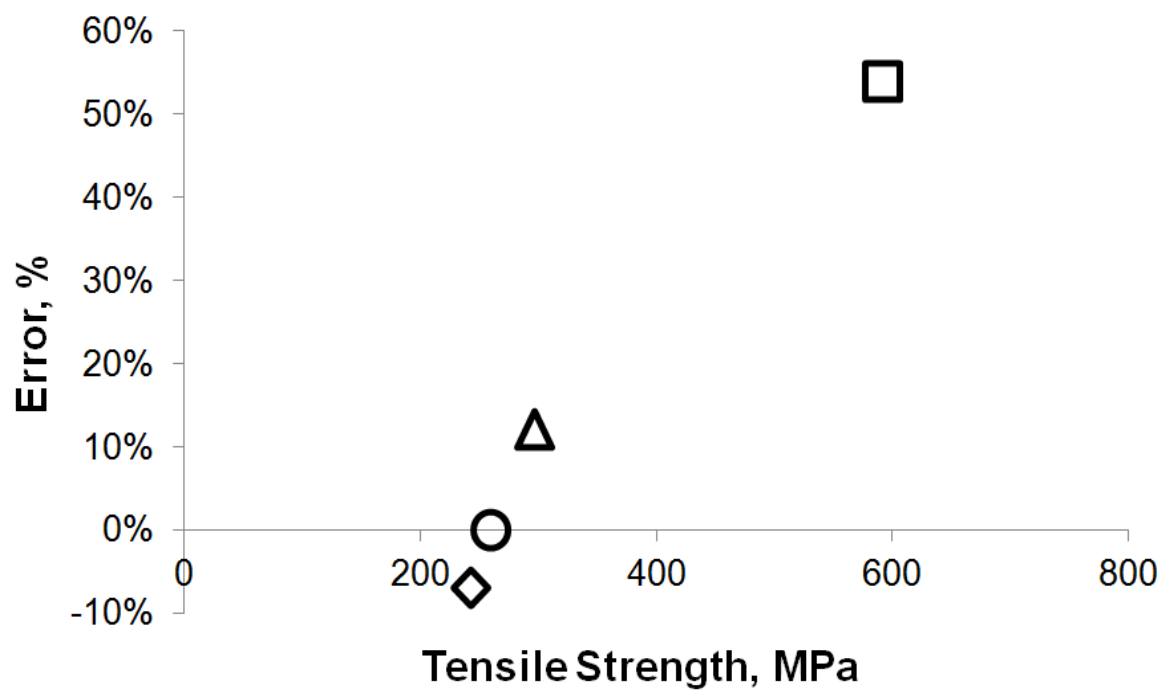

\section{口Matl \#1 $\quad \Delta$ Matl \#2 $\quad$ Matl \#3 OMatl \#4}

Figure 3.16: Error of material candidates respect of experimental results.

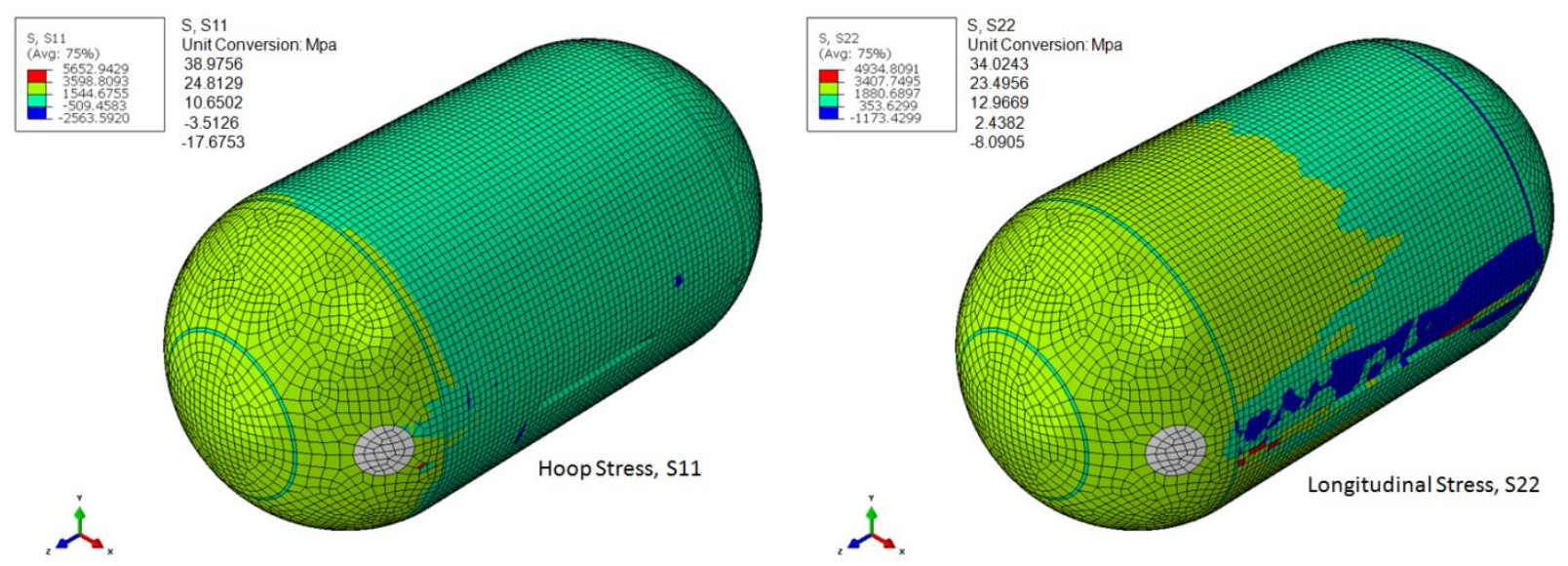

Figure 3.17: Contour of Hoop and longitudinal stresses obtained from FE results for constrained pressurization 


\subsubsection{Loading Case III: Axial Slippage Evaluation}

Table 3.4 presented previously summarized the definition of each model ID that represents the combinations of internal pressure $(\mathrm{Pi})$ and external pressure $(\mathrm{Pe})$ used for the slippage simulations. For instance, model ID of 55/40 represents the slippage model with a combination of external pressure of $0.3792 \mathrm{MPa}(55 \mathrm{psi})$ and internal pressure of $0.2758 \mathrm{MPa}(40 \mathrm{psi})$. Model $40 / 30$ has $0.2758 \mathrm{MPa}(40 \mathrm{psi})$ and 0.2068 $\mathrm{MPa}(30 \mathrm{psi}$ ) as external pressure and internal pressure, respectively. Model 30/20 has the combination of external pressure of $0.2068 \mathrm{MPa}(30 \mathrm{psi})$ and internal pressure of $0.1379 \mathrm{MPa}(20 \mathrm{psi})$. On the other hand, model 20/10 was designed to have external pressure of $0.1379 \mathrm{MPa}(20 \mathrm{psi})$ and internal pressure of $0.06895 \mathrm{MPa}(10 \mathrm{psi})$.

Table 3.7 illustrates the summary of holding resistance ratio $\left(\mathrm{P}_{\mathrm{e}} / \mathrm{P}_{\mathrm{i}}\right)$ obtained for friction coefficients of $0.15,0.19$, and 0.25 . Table 3.7 also shows the time (in seconds) at which the slippage occurred in the simulation. The slippage time was defined at the moment in which the plug had significant displacement change (a change of slope by more than $25 \mathrm{~mm}$ per second was adopted as indicator of axial slippage). The material properties used to run slippage tests correspond to Material \#4 described previously.

Table 3.7: Summary of holding resistance ratios for different friction coefficients.

\begin{tabular}{|c|c|c|c|c|c|c|}
\hline \multirow{2}{*}{ Model ID } & \multicolumn{2}{|c|}{$\mu=0.15$} & \multicolumn{2}{c|}{$\mu=0.19$} & \multicolumn{2}{c|}{$\mu=0.25$} \\
\cline { 2 - 7 } & $\mathrm{Pe} / \mathrm{Pi}$ & Time & $\mathrm{Pe} / \mathrm{Pi}$ & Time & $\mathrm{Pe} / \mathrm{Pi}$ & Time \\
\hline $55 / 40$ & - & 8 & 0.8163 & 20 & 0.9816 & 31 \\
\hline $40 / 30$ & - & 8 & 0.8108 & 18 & 0.9836 & 31 \\
\hline $30 / 20$ & - & 9 & 0.8163 & 23 & 0.9756 & 31 \\
\hline $20 / 10$ & 0.6667 & 22 & 0.8696 & 29 & 0.9524 & 31 \\
\hline Average & 0.6667 & & 0.8283 & & 0.9733 & \\
\hline
\end{tabular}

Figure 3.18 shows the slippage plot of the models with friction coefficient of 0.15 . The results show that models identified as $30 / 20,40 / 30$, and 55/40, with friction coefficient of 0.15 slipped as soon as the external pressure was applied on the plug. Therefore, they are not available to be calculated in terms of holding resistance ratios. The model 20/10 with friction coefficient of 0.15 slipped at $22^{\text {nd }}$ second and had a holding resistance ratio of 0.6667 . 


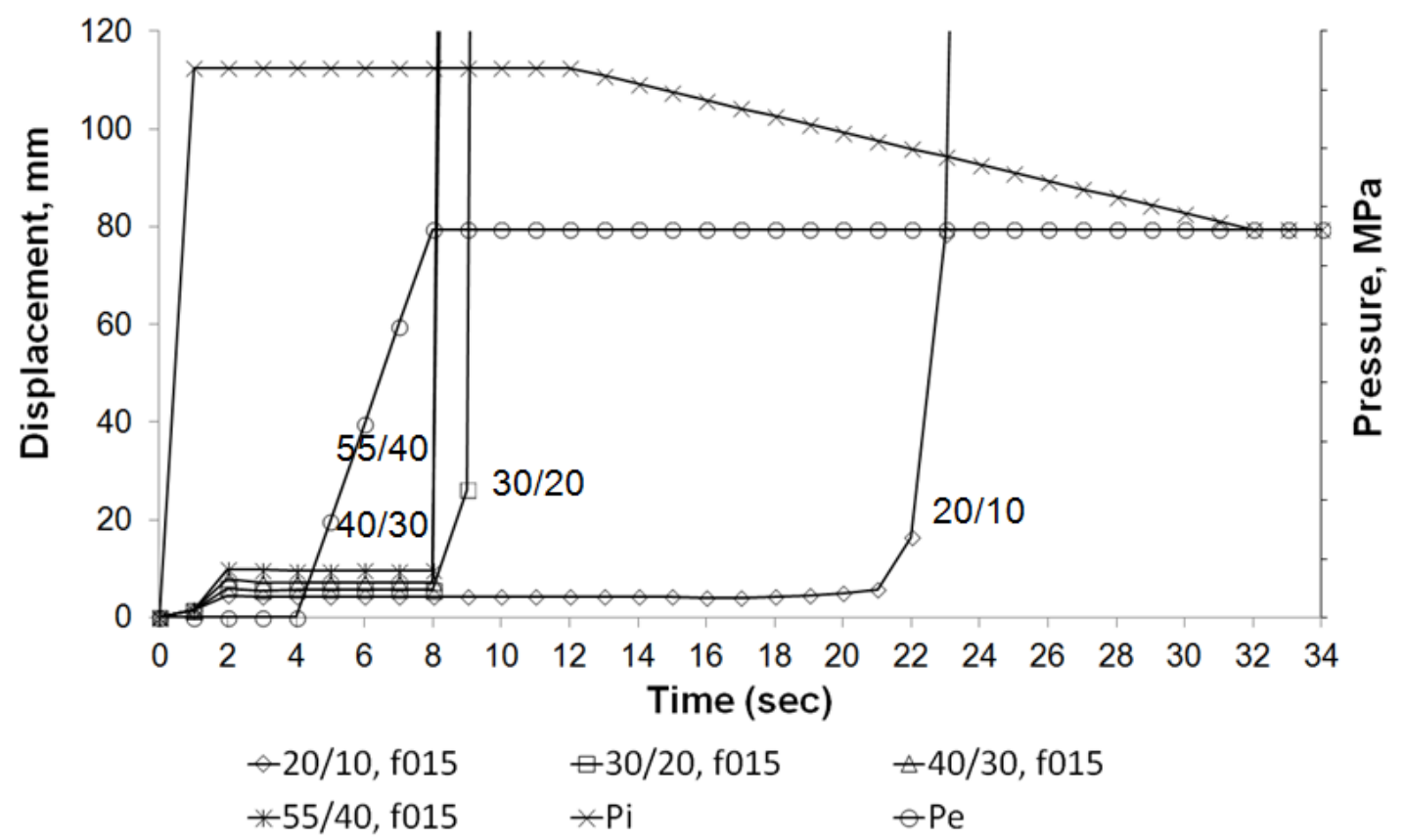

Figure 3.18: Slippage results of same friction coefficient $(0.15)$ with various loading combinations.

Figure 3.19 displays the slippage results corresponding to models with a friction coefficient of 0.19 . The average holding resistance for this case was 0.8283 . This value is nearly $3.5 \%$ higher than the average value of 0.80 obtained from experimental tests [59]. The loading combination of 20/10 displayed the highest holding resistance ratio with a value of 0.8696 . Models $30 / 20$ and $55 / 30$ resulted with same holding resistance ratios with values of 0.8163 , although their slippage timing was different $\left(20^{\text {th }}\right.$ and $23^{\text {rd }}$ second, respectively). The lowest holding resistance corresponded to the case 40/30 which produced a value of 0.8108 .

Figure 3.20 show the results corresponding to models with friction coefficient of 0.25 . As illustrated in the plots, there was no significant slippage for combinations $40 / 30$, $30 / 20,20 / 10$, and 55/40. The results obtained for friction coefficients 0.15 and 0.19 suggest that the holding resistance seems to be a function of the combination of pressures used to induce the slippage. That is, the higher the level of pressures (such as in the case of $55 / 40$ or $40 / 30$ ), the sooner the occurrence of slippage. In the other side, the lower the magnitude of the pressures, the later the slippage occurred. A similar trend can be observed in the case of $\mu=0.25$, where the plug did not slip significantly 
(less than $10 \mathrm{~mm}$ ) and just reached a new equilibrium position when the two acting pressures remained equal as illustrated in Figure 3.20.

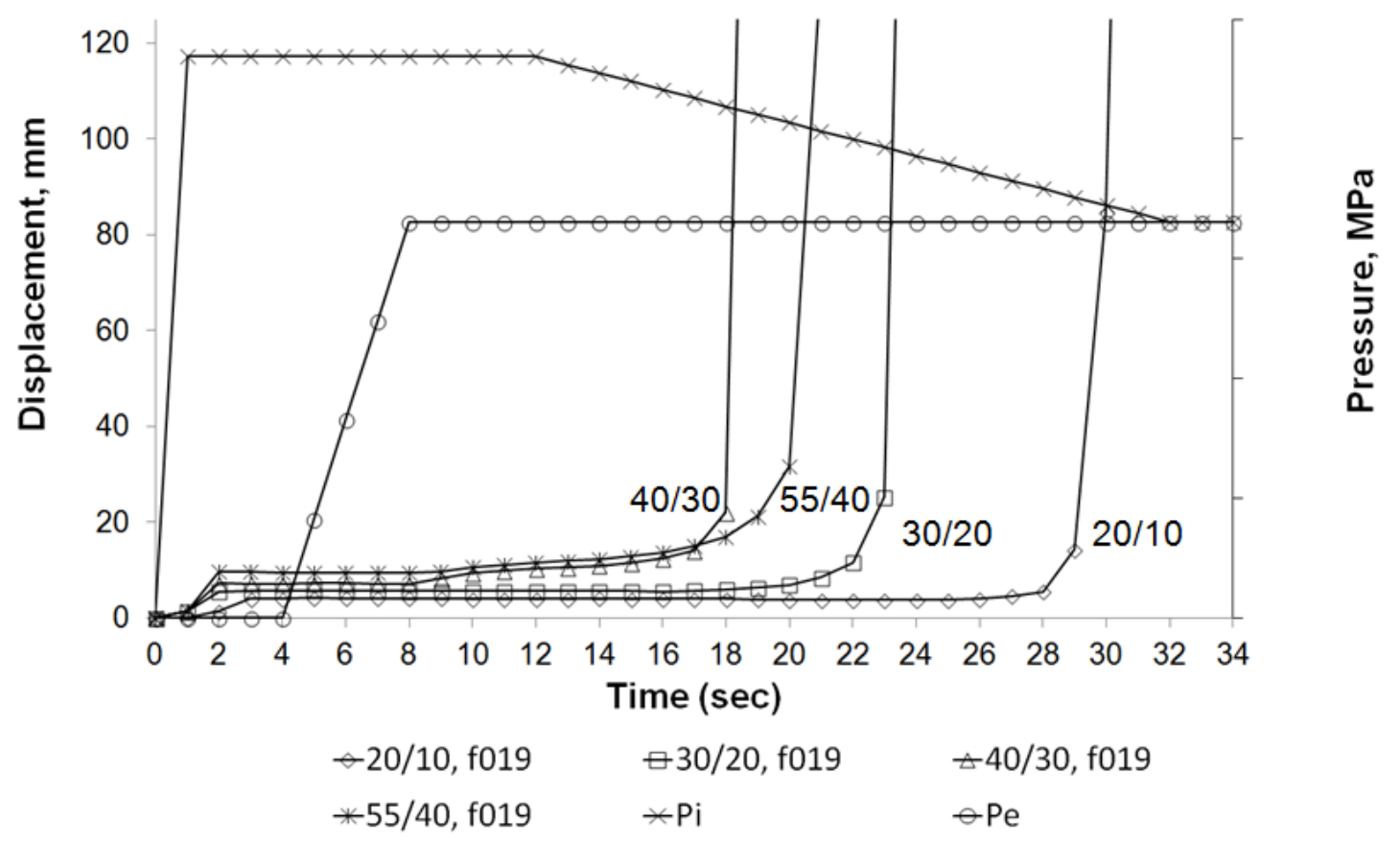

Figure 3.19: Slippage results of same friction coefficient $(0.19)$ with various loading combinations.

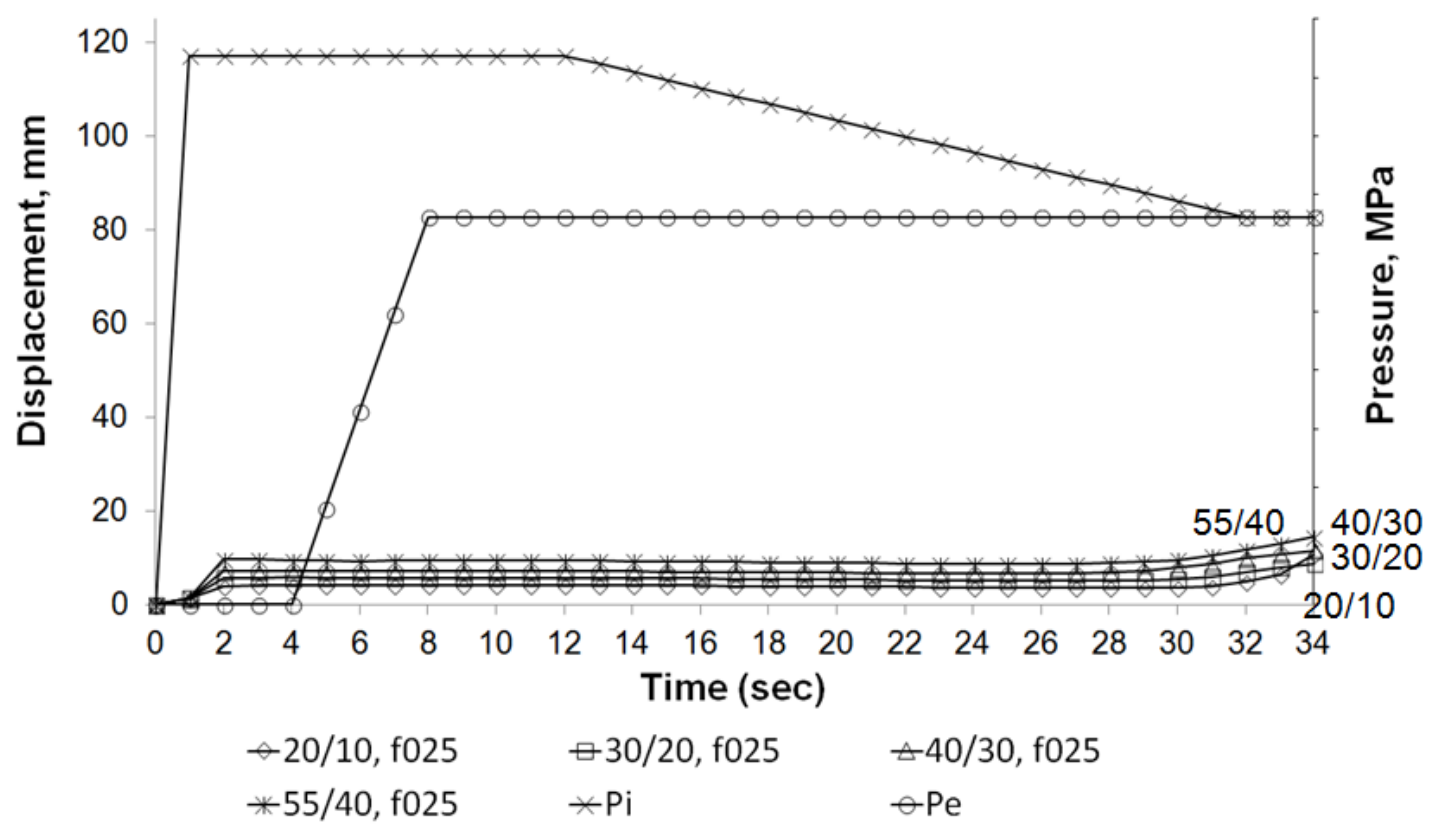

Figure 3.20: Slippage results of same friction coefficient $(0.25)$ with various loading combinations. 
Figure 3.21 displays the slippage results for models with the same loading combination (55/40) and different friction coefficients (0.15, 0.19, and 0.25). The 55/40 model with $\mu=0.15$ did slip as soon as the external pressure was applied. The model with $\mu=0.19$ resisted up to the $20^{\text {th }}$ second and the slipped. The model with $\mu=0.25$ moved nearly $15 \mathrm{~mm}$ before reaching a new equilibrium position. For this last case, it is safe to say that the plug did not slip within the time window considered for the analysis.

Figure 3.22 shows sequences of slippage corresponding to the $55 / 40$ model with friction coefficients of $0.15,0.19$, and 0.25 . Model with friction coefficient of 0.15 and 0.19 have obvious displacement at $8^{\text {th }}$ and $20^{\text {th }}$ second, respectively. Figure 3.22 also shows the model with friction coefficient of 0.25 did not slip.

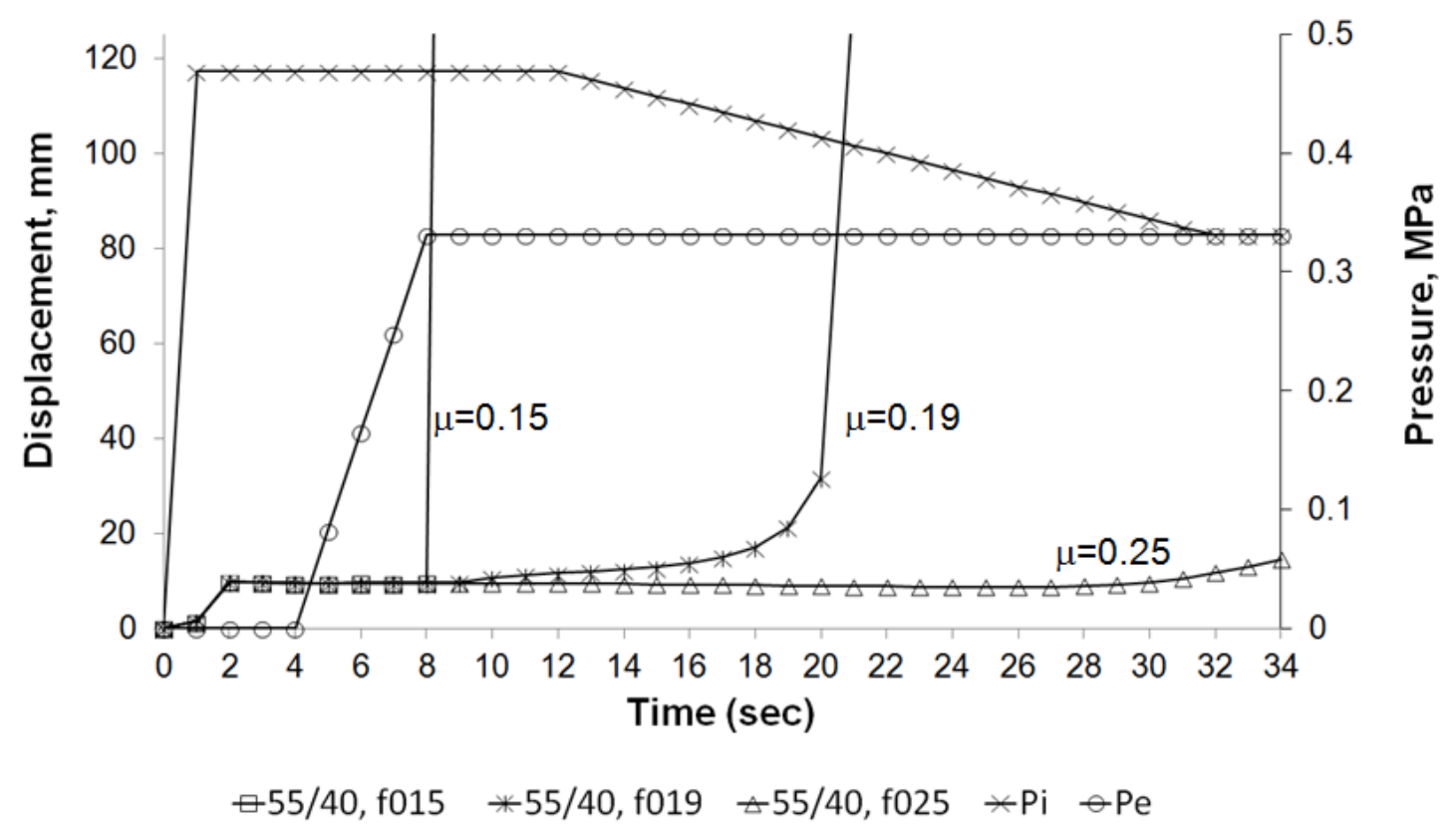

Figure 3.21: Slippage results of same loading combinations (55/40) with various friction coefficients. 


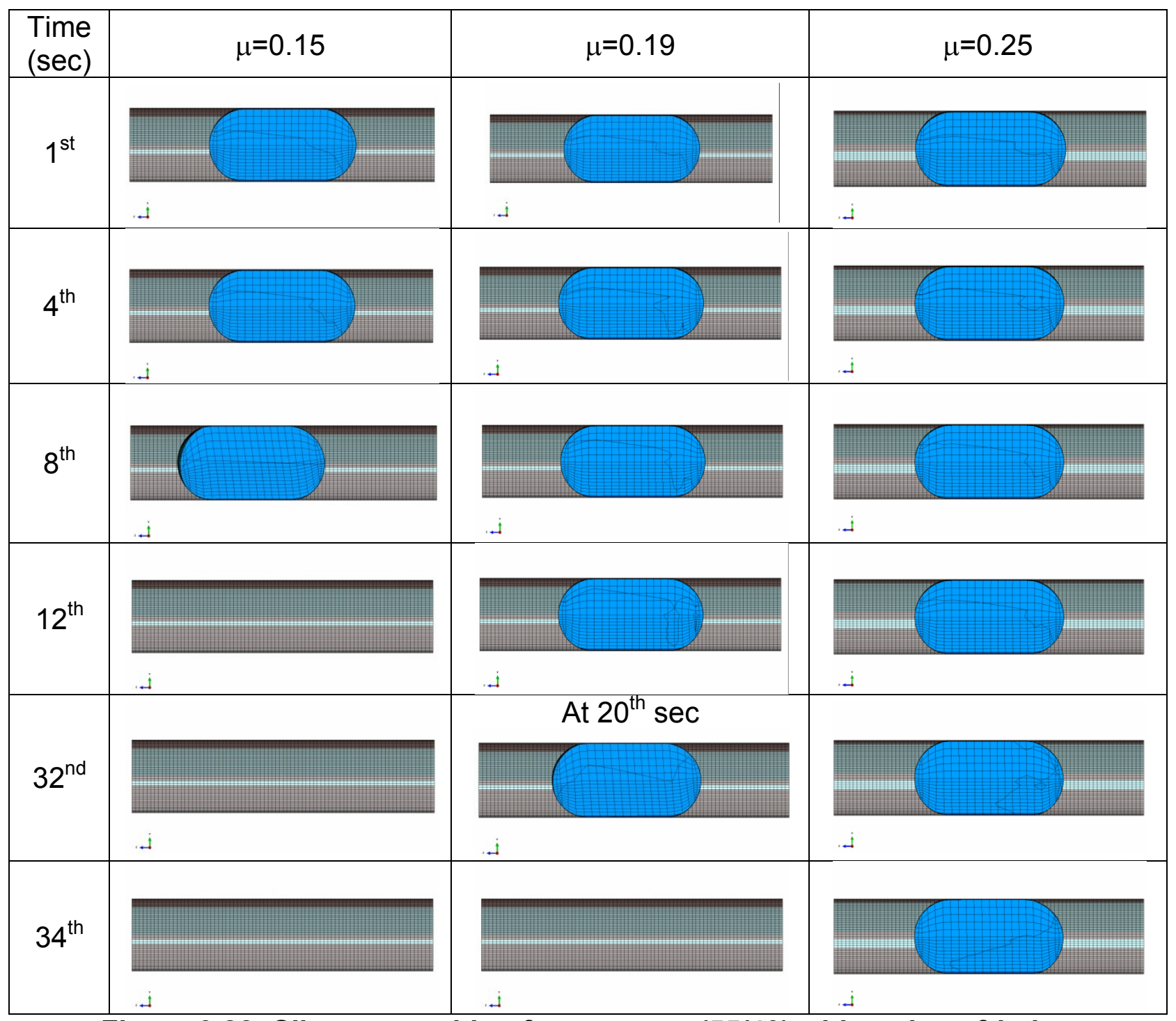

Figure 3.22: Slippage position for pressure (55/40) with various friction coefficients.

An additional case was run to verify the axial stability of the reduced-scale prototype when subjected to design pressures $\left(P_{i}=0.4688 \mathrm{MPa}\right.$ and $\mathrm{P}_{\mathrm{e}}=0.3309 \mathrm{MPa}$; $P_{i}=68 \mathrm{MPa}$ and $P_{e}=48 \mathrm{MPa}$, respectively) and different friction coefficients $(0.15,0.19$, 0.21 and 0.25 ). Figure 3.23 illustrates the results of evaluation of slippage for these cases. As expected, the lower friction coefficient, the earlier the occurrence of slippage as in the case of $\mu=0.15$. However, for $\mu=0.19, \mu=0.21$, and $\mu=0.25$, the plug remained stable. The case for $\mu=0.19$ was confirmed experimentally [59] and served as basis for comparison with the FE models developed for the full-scale prototype described in the next chapters. The results of FE models with friction coefficients of $0.15,0.21$, and 0.25 
could not be validated with experimental results, but provided an estimation of the magnitude of the friction coefficients to either induce or avoid slippage of the plug. The techniques and features developed and implemented in the FE modeling of the reduced-scale prototype served as a basis for the development of the FE simulation of the full-scale prototype.

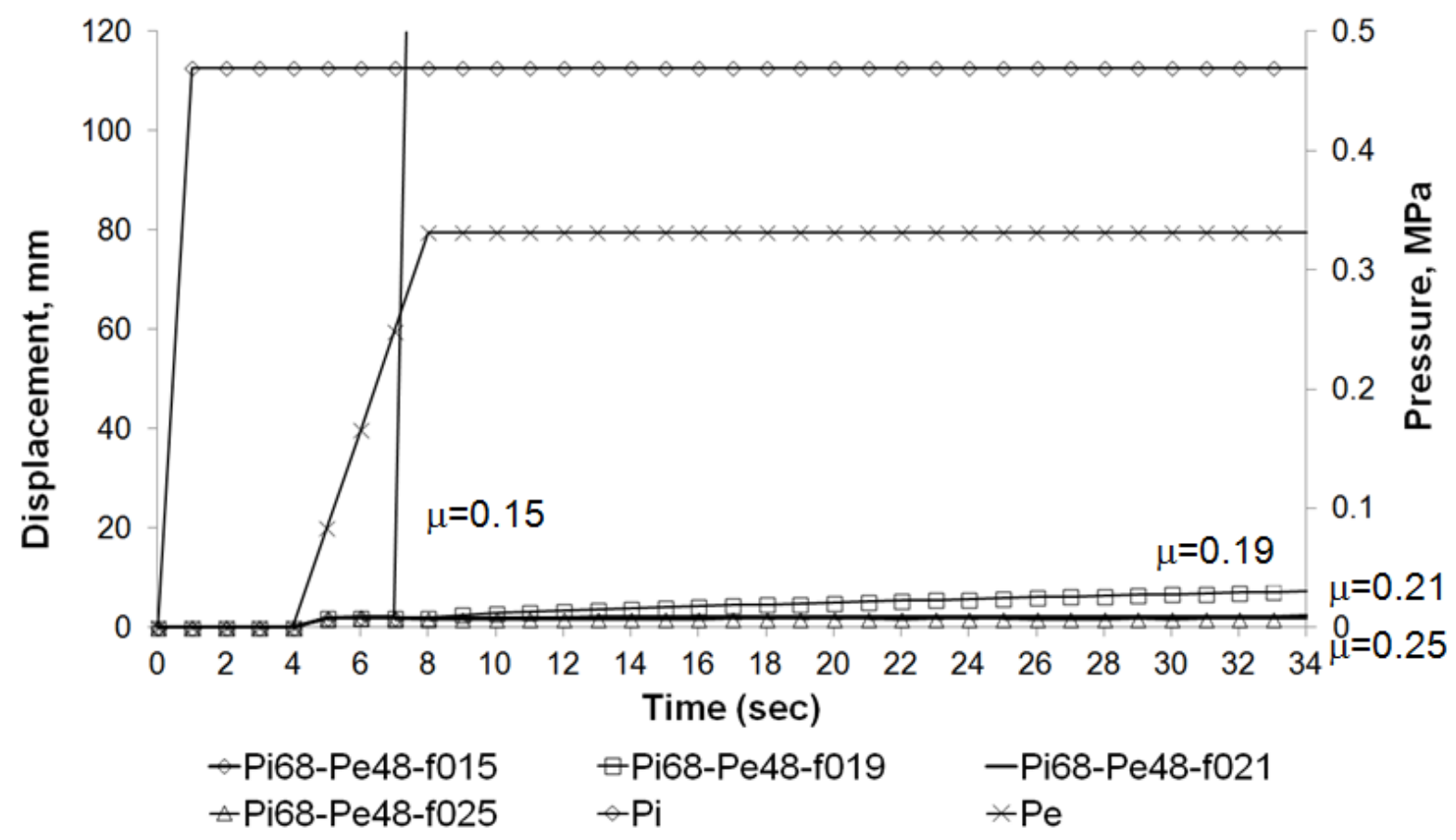

Figure 3.23: Slippage results for design pressure (68/48) with various friction coefficients.

\subsection{Summary}

Different FE models were developed for simulation of the behavior of a reducedscale prototype under different testing and loading conditions. The actual tri-layer structural membrane was replaced in the FE models by an equivalent single layer representative, in terms of mass and thickness, of the actual tri-layer system. The stiffness and maximum strength were adjusted based on available experimental results. A simplified approach for deployment and initial inflation was created for placing the inflatable inside the tunnel. 
Convergence studies indicated that a FE model of the reduced-scale prototype with at least 10000 elements will converge to the theoretical values of stresses corresponding to unconstrained pressurization. Material \#4 was chosen based on results of constrained elongation tests and will be used as the material for the development of FE model of the full-scale prototype.

The stress evaluation for unconstrained pressurization showed that the model can approximate to the theoretical values with a maximum error of $1.60 \%$. On the other hand, the stress evaluation for constrained pressurization showed that the hoop and longitudinal stresses in the hemispherical end-caps resulted $21 \%$ higher than the theoretical values, while the hoop and longitudinal stresses in the cylindrical portion of the plug resulted, as expected, considerably reduced (about $300 \%$ ) due to the confining effect of the tunnel.

The axial stability of the inflatable positioned in the tunnel was evaluated for different friction coefficients and pressure combinations. The model with friction coefficient of 0.19 displayed a holding resistance ratio of 0.8283 which is $3.5 \%$ higher than the result obtained experimentally $(0.80)$. This result shows the good correspondence between numerical and experimental results. 


\section{Full-Scale Prototype: FE Model Initial Preparation}

\subsection{Introduction}

This chapter presents the initial steps that were necessary for the generation of the FE model of the full-scale prototype. Dimensions and the process of creating each part are presented. Models of the key components needed to achieve a realistic behavior including the inflatable structure, tunnel section, and the folding components are described. The simulation features and material properties developed and implemented in the FE model of the reduced-scale prototype are applied to the fullscale FE models.

\subsection{Dimensions of Full-Scale Prototype}

The full-scale prototype consists of a cylinder with two hemispherical end-caps (Figure 4.1). The cylinder has a diameter of $4937.3 \mathrm{~mm}$ (194.48 in) and length of 4640.6 $\mathrm{mm}$ (182.70 in). Each hemispherical end-cap has diameter of $4937.3 \mathrm{~mm}$ (194.48 in) and include three partitions on its surface. Each partition is delimited by ropes that connect longitudinal webbings that terminate at different locations on the circular perimeter in order to avoid overcrowding of webbing at the apex of each hemispherical end-cap, as illustrated in Figure 4.2. The ropes have a diameter of $50.8 \mathrm{~mm}$ (2 in) and are made of Vectran fibers. The perimeter of full-scale prototype is designed to be larger than tunnel's intentionally. This oversizing is added in order to account for potential manufacturing imperfections as well as for unforeseen irregularities that the plug will have to conform around to achieve an acceptable level of sealing. In this study the amount of oversizing was kept constant at approximately $6 \%$.

Two aluminum fittings are assembled as part of the fill and air release ports in one of the hemispherical end-caps. The fill port fitting is $355.60 \times 355.60 \mathrm{~mm}$ (14"x 14") with a circular opening with diameter of $203.20 \mathrm{~mm}$ (8 in). The air release fitting is $254.00 \times 254.00 \mathrm{~mm}$ (10" x10") with an orifice of $101.6 \mathrm{~mm}$ (4 in) diameter (Figure 4.2); the mass of each one of them is $13.6078 \mathrm{~kg}(30 \mathrm{lbs})$ and $6.8039 \mathrm{~kg}(15 \mathrm{lbs})$, respectively. The inflatable plug is constructed with the same tri-layer system as described in Chapter 3. The total mass of plug (including metallic fittings) is approximately $907.1847 \mathrm{~kg}(2000 \mathrm{lbs})$. 


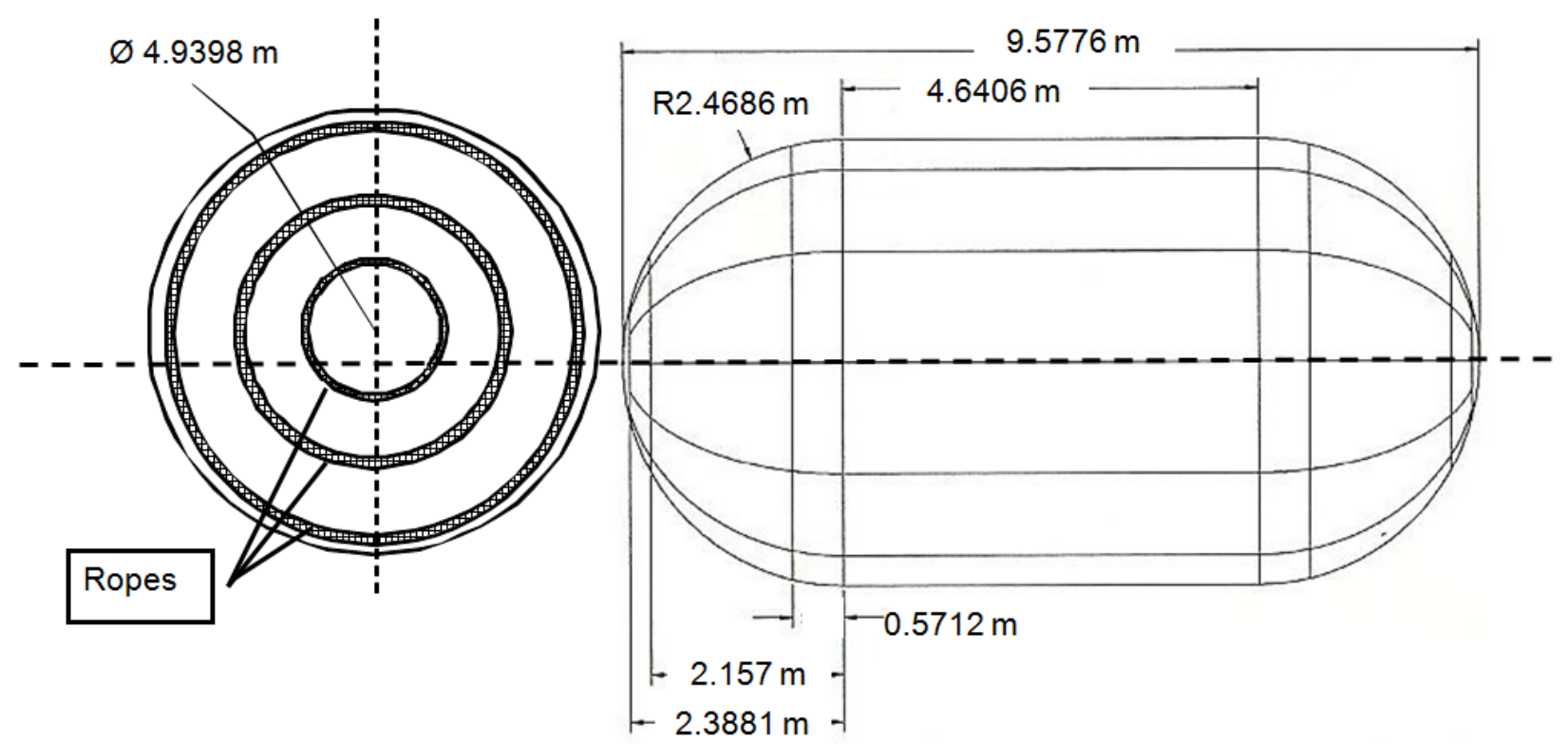

Figure 4.1: Nominal Dimensions of Full-Scale Prototype.

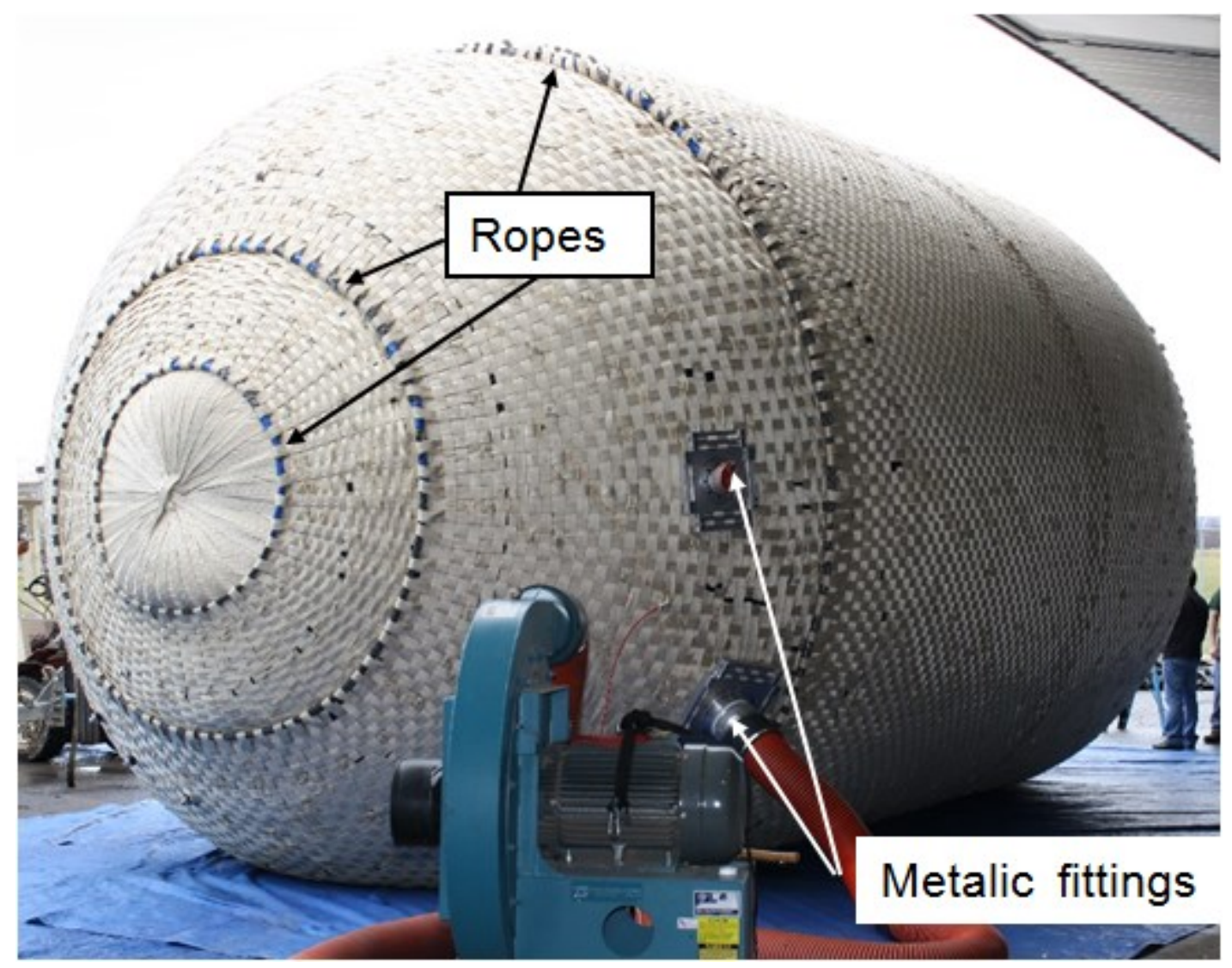

Figure 4.2: Isometric View of Actual Full Scale Prototype. 


\subsection{Generation of FE Geometry}

The FE geometry of the inflatable plug was created using three-dimensional shells through Abaqus/CAE. Figure 4.3(a) shows how the plug was initially partitioned into several auxiliary surfaces and edges. The purpose of creating these auxiliary surfaces and edges is to delimit the position of fitting areas, ropes, internal chamber surfaces, folding surfaces and folding lines at the cylindrical region of the plug. Figure 4.3(b) shows the mesh configuration of the full-scale prototype. M3D4 membrane elements were assigned to represent the macro-mechanical behavior of the full scale prototype. The fittings are modeled by R3D4 rigid elements.
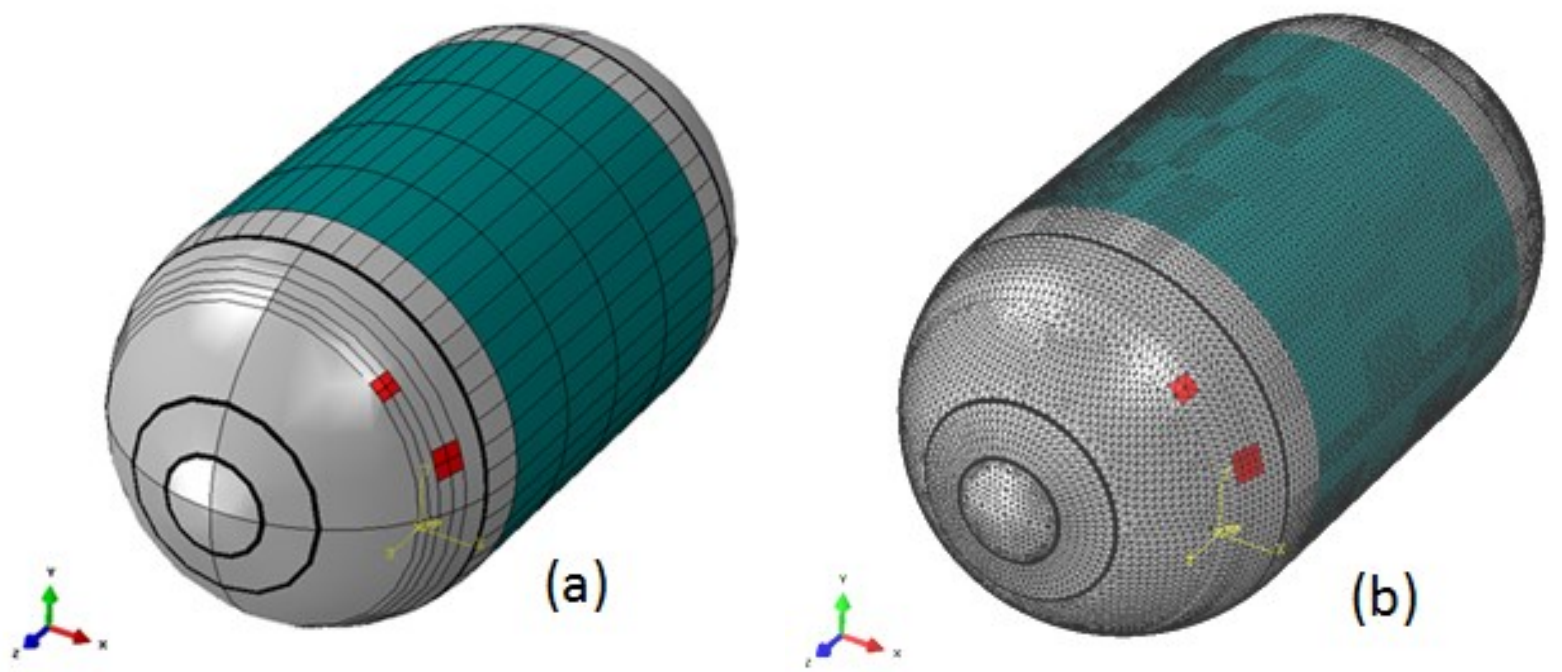

Figure 4.3: (a) Initial Geometry and (b) Mesh Configuration.

\subsubsection{Stress Evaluation}

In this section, the longitudinal stress and hoop stress of the full scale prototype are evaluated analytically and numerically in order to assess the accuracy of the FE model. The inflatable plug was assumed to be subjected to an internal pressure of $0.1172 \mathrm{MPa}(17 \mathrm{psi})$. The total thickness of fabric is $7.7978 \mathrm{~mm}(0.307 \mathrm{in})$ and dimensions of the inflatable plug were shown in Figure 4.1.

In order to have a reference point, the analytical solutions for the hoop stress and longitudinal stress for hemispherical end cap and the cylindrical region were obtained for an unconstrained configuration using classical equations for thin-walled structures under internal pressure. Theoretically, the hoop stress $\left(S_{11}\right)$ and longitudinal stress $\left(S_{22}\right)$ 
at hemispherical caps are the same and were calculated with Equation 3.1 (Chapter 3). On the other hand, the Equation 3.2 and 3.3 are applied to calculate the hoop and longitudinal stresses, respectively, in the cylindrical region.

Figures 4.4 and 4.5 present the contour plots of the distribution of hoop stress and longitudinal stress when the model was meshed with approximately 30,000 elements (20,000 DOF). The average hoop $\left(S_{11}\right)$ and longitudinal $\left(S_{22}\right)$ stresses of FE model were captured at several nodes located on the hemispherical end-caps and on the cylindrical region of the plug and represented by the small circles highlighted in Figure 4.4. At the hemispherical end-cap, the theoretical value for both hoop stress and longitudinal stress is $18.55 \mathrm{MPa}$ (2690.93 psi). The simulation result shows that the average stress is $18.23 \mathrm{MPa}$ (2644.61 psi), which is $1.72 \%$ lower than the theoretical value.

From Figures 4.4 and 4.5, it is seen that, as expected, the hoop stress and longitudinal stress in the cylindrical region are distributed differently. The theoretical hoop stress is $37.11 \mathrm{MPa}(5381.86 \mathrm{psi})$ and the simulation predicts an average hoop stress of $36.11 \mathrm{MPa}(5237.64 \mathrm{psi})$ which has an error of $2.69 \%$. On the other hand, the longitudinal stress $\left(S_{22}\right)$ in the cylindrical region has same value as the longitudinal or hoop stress in hemispherical end caps, which is $18.55 \mathrm{MPa}$ (2690.93 psi) and $18.01 \mathrm{MPa}(2612.51 \mathrm{psi}$ for the theoretical and numerical results, respectively. The error in this case is $2.91 \%$. Table 4.1 illustrates a summary of the stress evaluation and Appendix B summarizes the analytical calculations of both longitudinal and hoop stresses.

Table 4.1: Stress evaluation results of unconstrained full-scale prototype.

\begin{tabular}{|c|c|c|c|c|}
\hline \multirow{2}{*}{ Full Scale Model } & \multicolumn{2}{|c|}{ Front and Rear Spherical End Caps } & \multicolumn{2}{c|}{ Cylindrical Region } \\
\cline { 2 - 5 } & \multicolumn{2}{|c|}{$\mathrm{SPa}$} & $\mathrm{S} 11$ & $\mathrm{SP} 22$ \\
\cline { 2 - 5 } & 18.5533 & 18.5533 & 37.1066 & 18.5533 \\
\hline Theoretical Values & 18.3675 & 18.1004 & 36.1123 & 18.0126 \\
\hline FE Results & 0.1376 & 0.3651 & 0.9997 & 0.5407 \\
\hline Difference & \multicolumn{2}{|c|}{$1.72 \%$} & $2.69 \%$ & $2.91 \%$ \\
\hline \% of Error & \multicolumn{2}{|c|}{}
\end{tabular}




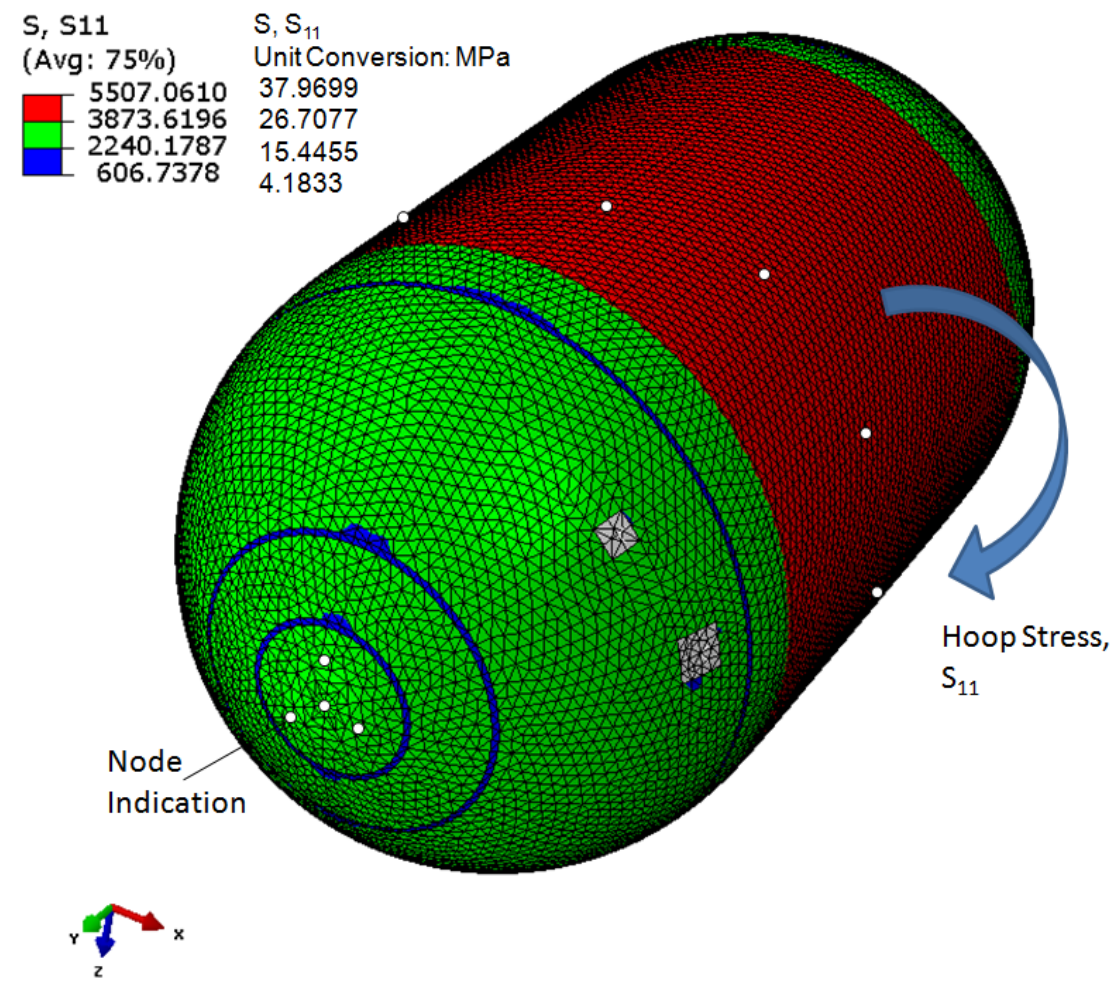

Figure 4.4: Hoop Stress of Model with 30000 elements (20000 DOF).

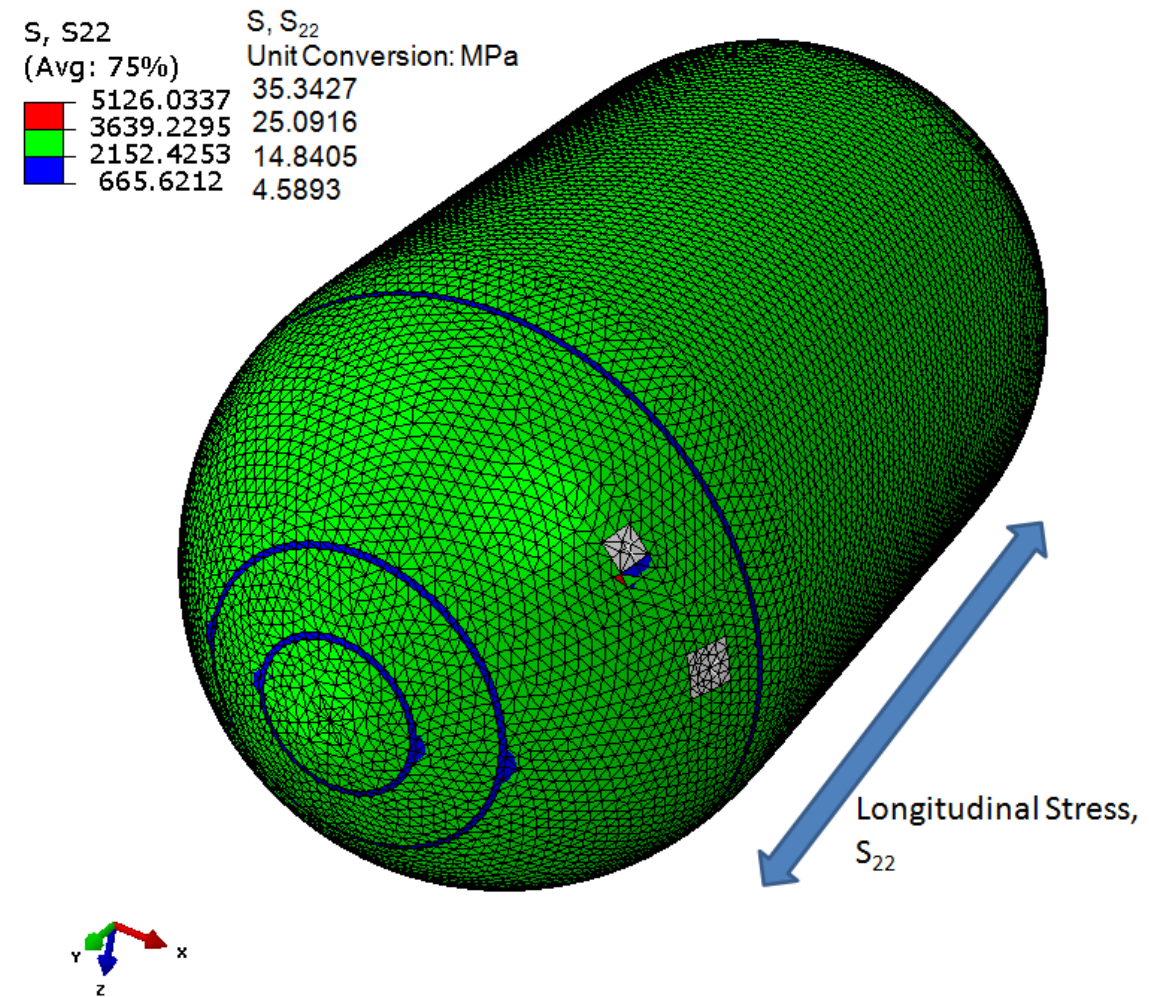

Figure 4.5: Longitudinal stress of Model with 30000 elements (20000 DOF). 


\subsubsection{Mesh Convergence Study}

The hoop stress $\left(S_{11}\right)$ in the cylindrical region for an unconstrained pressurization was chosen as control parameter for evaluation of mesh convergence. The control nodes are located in the $X-Z$ plane perpendicular to the longitudinal $Y$ axis of the cylindrical region of the plug as illustrated in Figure 4.4. The mesh density of the models was refined progressively from approximately 5000 30000 elements (16000 to 89100 DOF). Table 4.2 shows that a model with nearly 20000 elements (30000 DOF) has percentage of error of $2.69 \%$ when compared to the theoretical value of hoop stress. This error was considered acceptable taking into account that for the next mesh density with nearly 30000 elements, the computational time increased $33 \%$ for a small decrease in the percentage of error from $2.69 \%$ to $2.47 \%$. Figure 4.6 shows the convergence of the model to the theoretical solution as the mesh was refined by increasing the number of elements. Based on the results of the convergence study, a model with at least 20000 elements ( 30000 DOF) was used for further analyses.

Table 4.2: Summary of convergence study using hoop stress as control parameter.

\begin{tabular}{|l|l|c|c|c|c|}
\hline \multirow{2}{*}{$\begin{array}{l}\text { Full Scale } \\
\text { Prototype }\end{array}$} & Number of Elements & 5496 & 10888 & 19980 & 29692 \\
\cline { 2 - 6 } & Number of DOF & 8256 & 16344 & 29982 & 44550 \\
\hline \multirow{3}{*}{$\begin{array}{l}\text { Hoop Stress } \\
\left(S_{11}\right) \text { at }\end{array}$} & Theoretical Value, MPa & 37.1066 & 37.1066 & 37.1066 & 37.1066 \\
\cline { 2 - 6 } $\begin{array}{l}\text { Cylindrical } \\
\text { Region }\end{array}$ & $\begin{array}{l}\text { Average Hoop Stress }\left(S_{11}\right), \\
\mathrm{MPa}\end{array}$ & 34.8297 & 35.8303 & 36.1069 & 36.1890 \\
\cline { 2 - 6 } & Difference Cost & 2.2769 & 1.2763 & 0.9997 & 0.9176 \\
\cline { 2 - 6 } & Error \% & $\begin{array}{l}\text { Computational } \\
\text { (minutes) }\end{array}$ & 2 & 3 & 4 \\
\hline
\end{tabular}




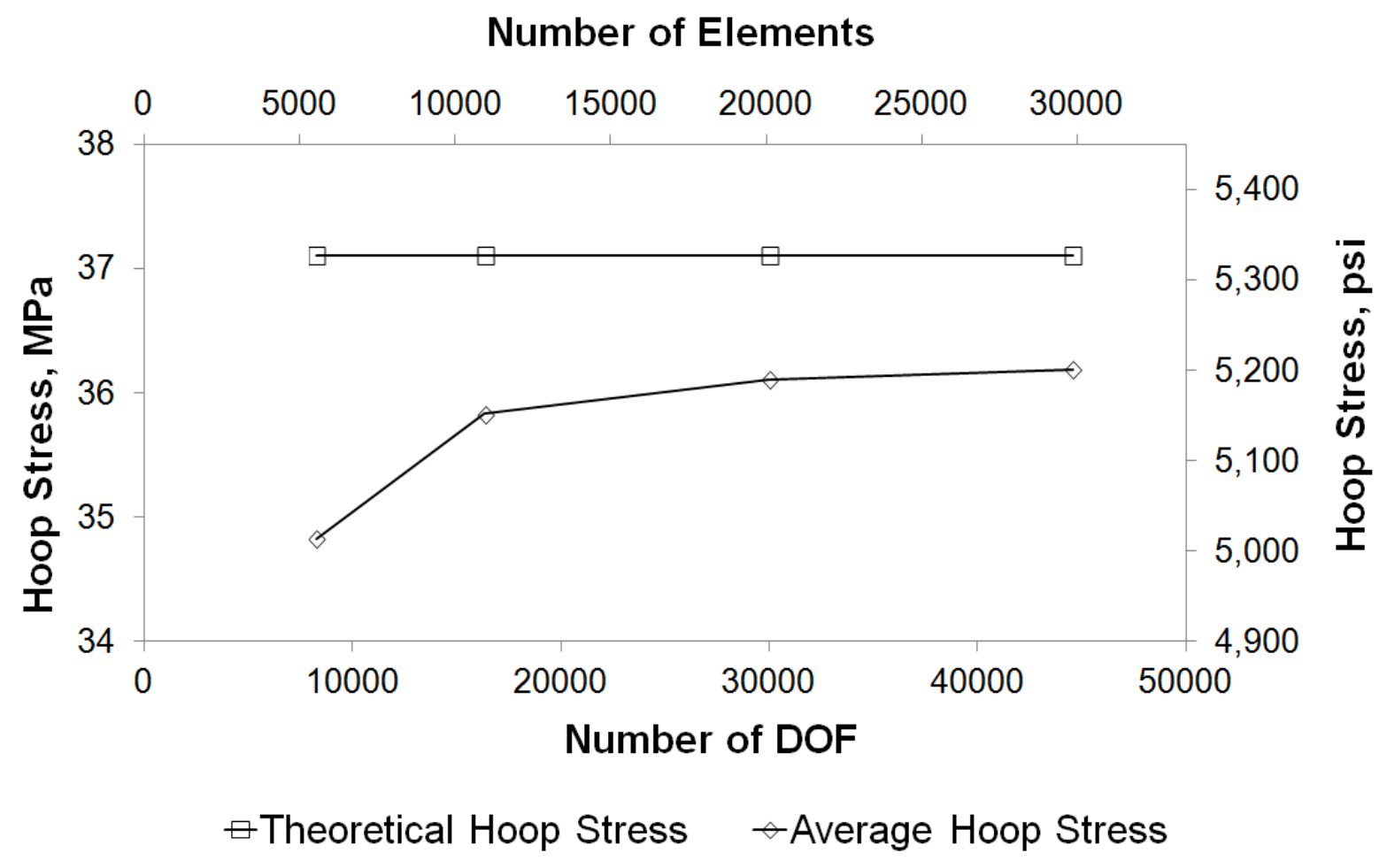

Figure 4.6: Plot of Mesh Convergence.

\subsection{Geometries of Tunnel, Base, and Rotational Plates.}

The tunnel section in which the inflatable plug will be placed is assumed to be non-deformable. The mesh consists of 5670 rigid elements of the type R3D4 [42-43]. Figure 4.7 shows the 3D geometry and the meshed configuration of the tunnel used for all the analyses. Figure 4.8 shows a typical cross section used for the analyses indicating also the space used for storage of the folded plug.

During the folding process described in the next chapters, the inflatable plug will interact with a flat base and auxiliary rotational plates created to simulate the folding process. Geometries and meshes of these auxiliary elements are illustrated in Figure 4.9 and 4.10. Similarly to the tunnel section, these surfaces are considered nondeformable and were meshed with R3D4 rigid elements. 

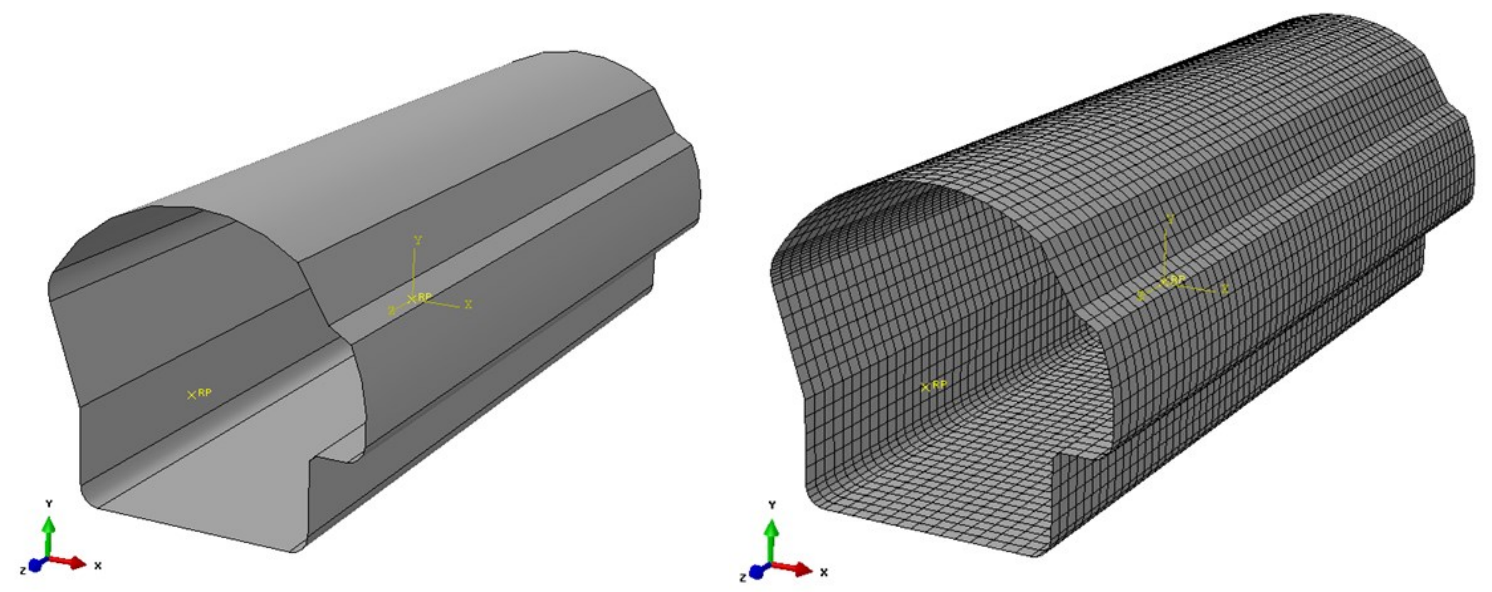

Figure 4.7: Geometry (left) and Mesh Configuration (right) of Tunnel.

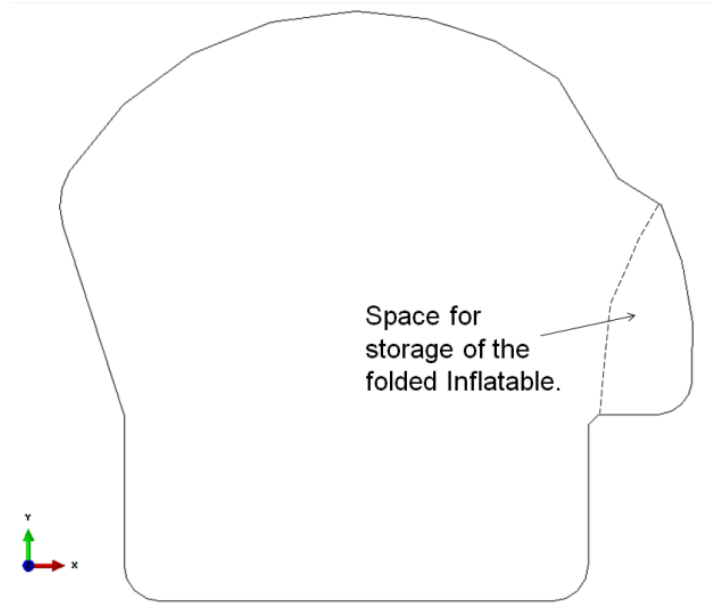

Figure 4.8: Typical tunnel cross section.

Rigid elements were assigned to the tunnel section, base, and rotational plates in order to minimize the simulation time since there will be no element-level calculation performed for rigid elements and assigned rigid elements do not contribute to the mass of the rigid body. There are several advantages using rigid elements for the auxiliary surfaces:

(i) They are used to represent the surfaces of contact of rigid bodies

(ii) They are used to simulate multi-body dynamic cases

(iii) They are attachable to the deformable elements, and

(iv) They can provide constraint on parts in a model. 
Figure 4.11 displays all the geometries including the inflatable plug, tunnel, auxiliary base, and auxiliary rotational plates that involved in the development of the FE model of the full-scale prototype.
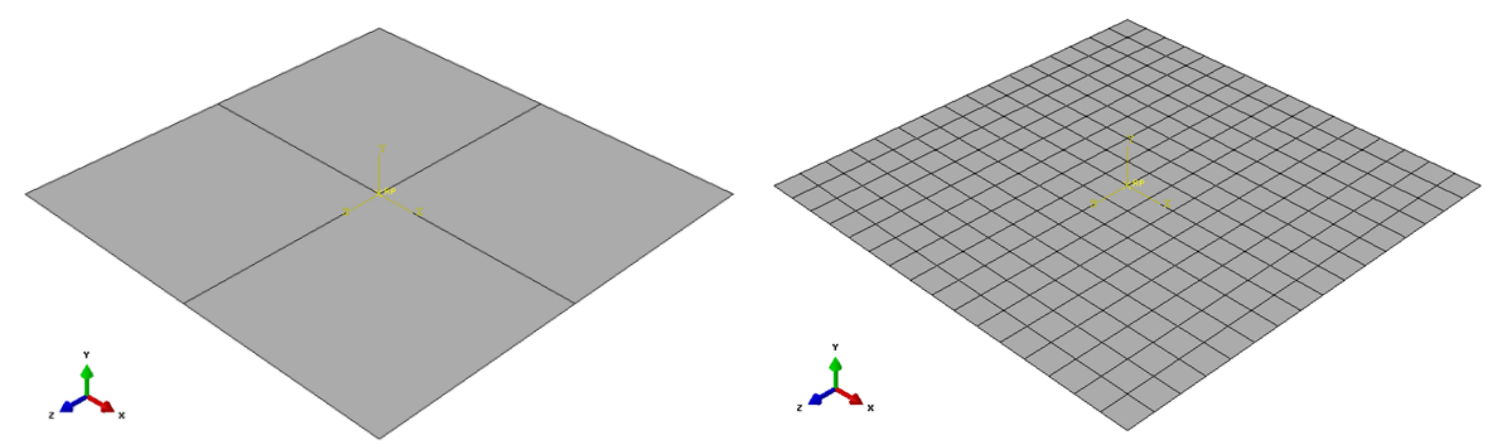

Figure 4.9: Geometry (left) and Mesh Configuration (right) of Base.
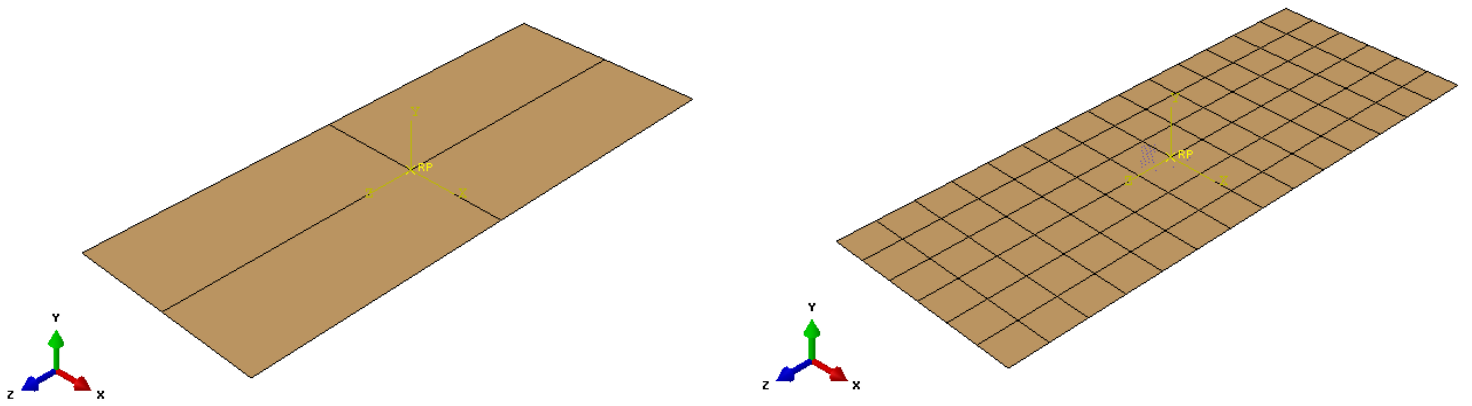

Figure 4.10: Geometry (left) and Mesh Configuration (right) of Rotational Plate.

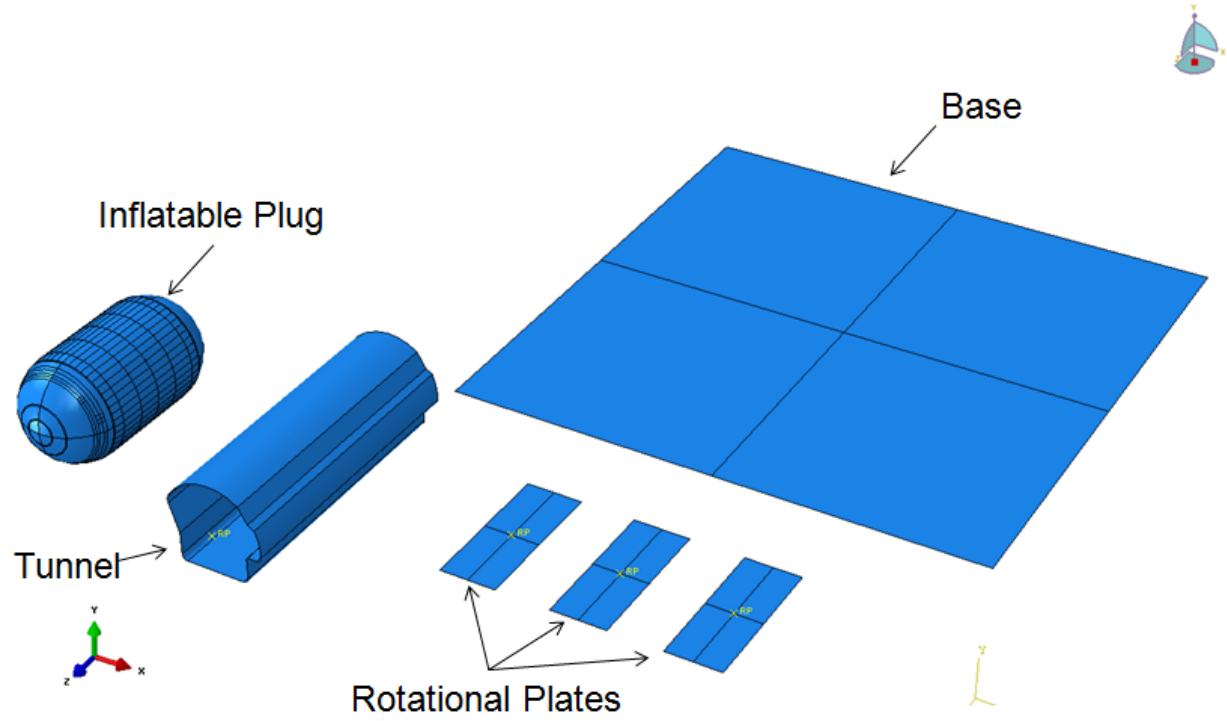

Figure 4.11: Components of the FE model for full-scale prototype. 


\subsection{Summary}

The initial steps necessary for the creation of a FE model of the full-scale prototype were presented in this chapter. The different components of the FE model were created considering all the stages of development of the model that include folding, placement in the storage area, relaxation, and final deployment of the inflatable plug.

All the nodes and elements of meshed components have been renumbered through HyperMesh and prepared to be utilized with Abaqus Scripting in order to make the simulation work more efficient.

The results of convergence study indicated that a model with at least 20000 elements will be needed to obtain relatively accurately results in terms of stresses at a reasonable computational cost. 


\section{Full Scale Prototype: Folding Process}

\subsection{Introduction}

For the purposes of this work, folding is a process that transforms a flattened inflatable structure into a certain compact shape so that it can be situated in a particular place or position that simulates storage of the folded inflatable. There are several brands of commercial software tools that provide ready-to-use folding features for airbag applications in the automotive industry. Some examples of commercial brands include: EASi-CRASH and PAM-CRASH developed by ESI Group [64]; OASYS Primer developed by Oasys Ltd which is a software house of ARUP [65]; LS-INGRID and LSPrePost developed by LS-DYNA [66]; HyperCrash [67] and Radioss Pre-Simulation [68] developed by ALTAIR; VPG Safety Module developed by Engineering Technology Associates, Inc (ETA) [69], and TEC|FOLD developed by TECOSIM [70]. Most of these software tools can be used for folding airbags with simple shapes (typically a $2 \mathrm{D}$ flattened inflatable structure) using one or a combination of the following folding strategies $[67,69]$ :

- Tuck Fold: Define a new fold layer in between existing layers.

- Thick/Thin Fold: Create the folding by offsetting the layers accounting for the material thickness and radius.

- Simple Fold: Fold the layers about a specific folding line.

- Rolling Fold: Also known as spiral fold which rolls the layers about a specific center of rotation with user-defined radius.

- Zigzag Fold: Fold the layers in a "Z" shape.

Figure 5.1 illustrates the folding strategies used by the commercial software tools mentioned previously. The folding algorithms for replicating a $2 \mathrm{D}$ flatten inflatable structure are considered mature. 


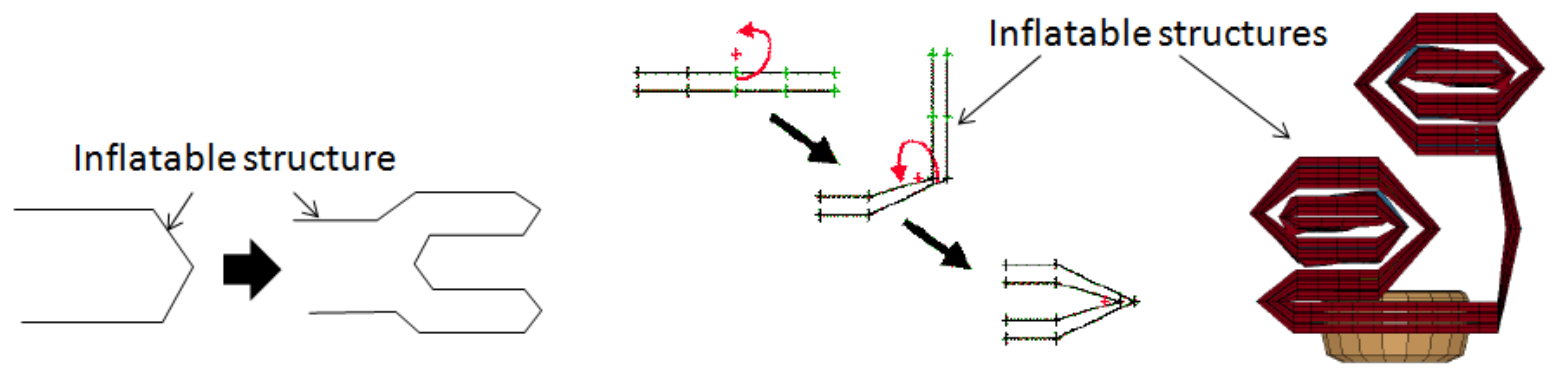

(a)

(b)

(c)

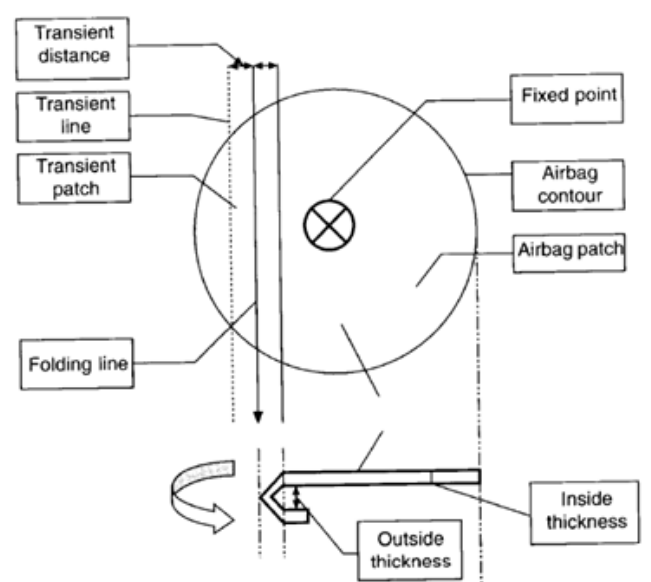

(d)

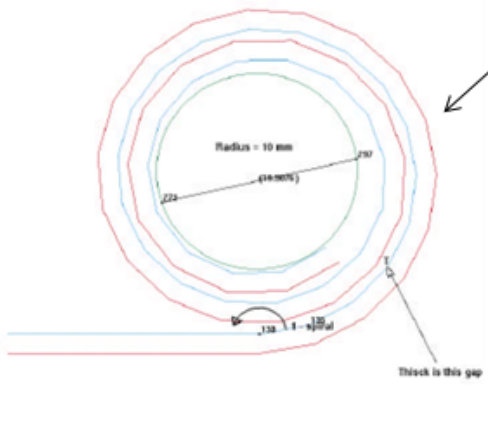

(e)
Inflatable structure

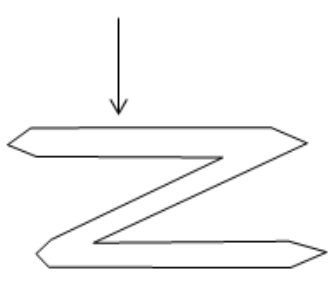

(f)

Figure 5.1: (a) Tuck fold, (b) Thin fold [71], (c) Thick fold [72], (d) Simple fold [73], (e) Rolling/Spiral fold [74], and (f) Zigzag fold

For more sophisticated applications, Tanavde et al. [75] developed a methodology called Initial Metric Method (IMM) to generate a flattened and folded mesh from a CAD geometry. The IMM consists of two types of meshes, a CAD reference mesh and a mapped/scaled/compressed mesh generated from a CAD mesh of the inflatable structure. The mapped/scaled/compressed mesh is used for inflation in the deployment simulation. The IMM uses the geometric difference of internal forces between the reference mesh and mapped mesh to compensate the mapped mesh in order to achieve the correct geometrical model. Zhang et al. [76] developed an automatic airbag modeling strategy using the IMM (Figure 5.2) and Park and Hong [77] use IMM to create the folded airbag implemented in their simulations as well. 


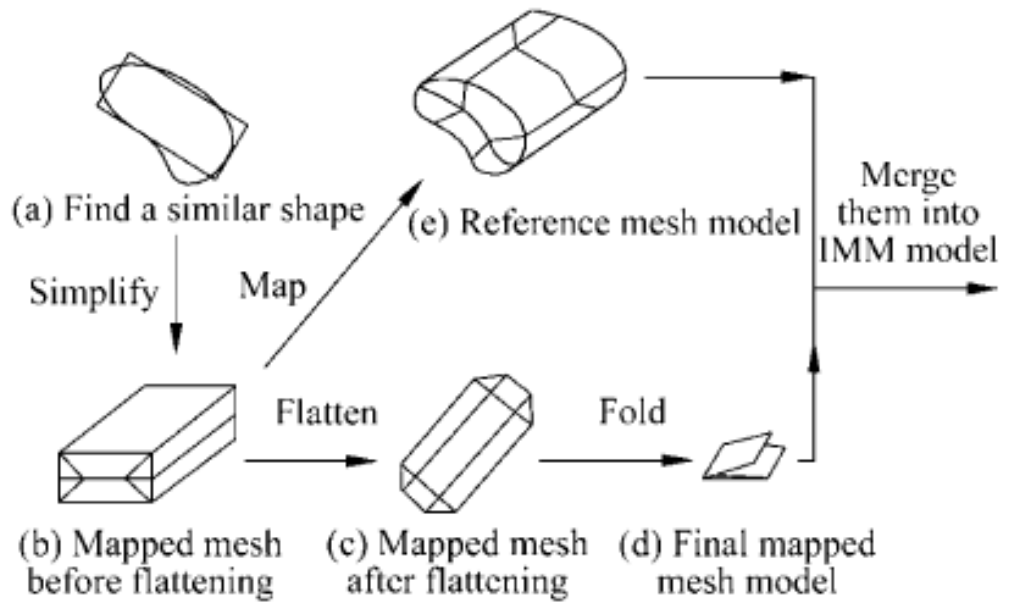

Figure 5.2: Automatic Modeling with IMM [76].

Beside the IMM approach, Cromvik [78] implemented the mathematical theory of Origami to flatten and fold an airbag when the geometry was too complex to fold with one of the above folding strategies (Figure 5.3). Cromvik approaches the folding by making a polyhedron approximation which is a mathematical Origami method that decomposes the folding patterns of a 3D geometry into a 2D flat shape [78]. The folding pattern is computed to be simple and able to restore close to the actual folds on the real inflatable [78].
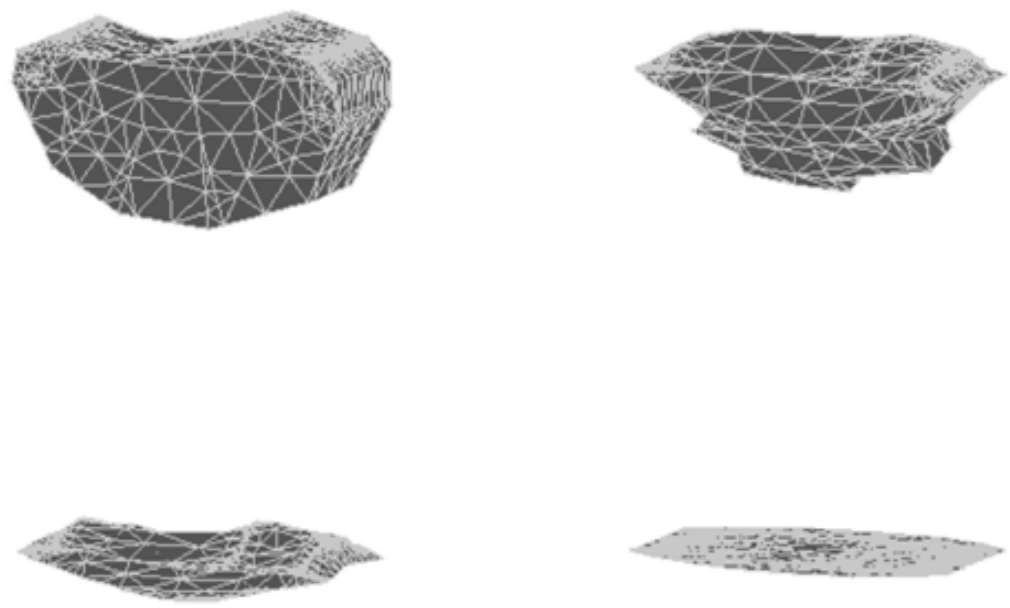

Figure 5.3: Folding sequence of the polyhedron approximation geometry [78].

Another strategy is the one proposed by Chawla et al. [79] who proposed a manual folding process using rigid planes (Figure 5.4). Similarly, Rieger [73] uses a rigid 
geometry to simulate the manufacturing process that includes folding of the inflatable structure (Figure 5.5).

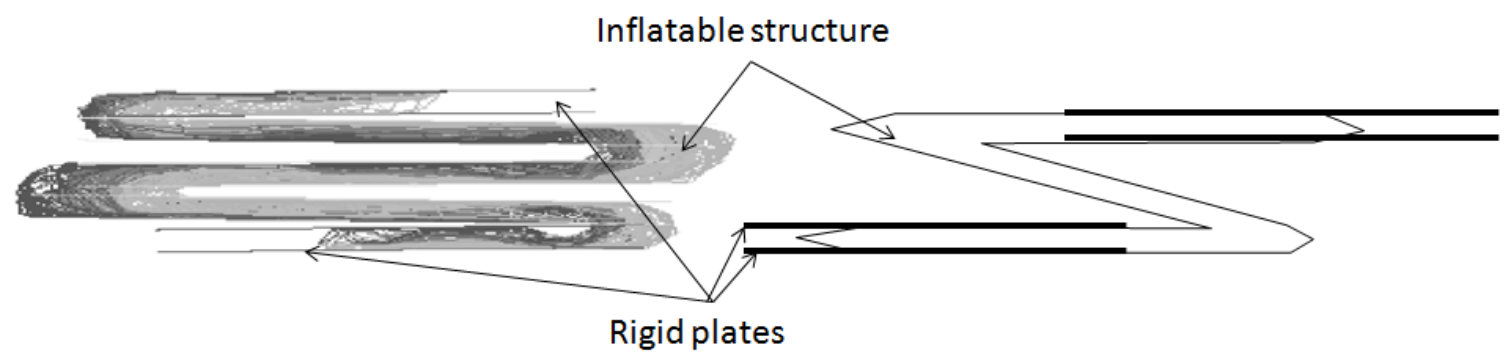

Figure 5.4: A zigzag fold using rigid plates [79].

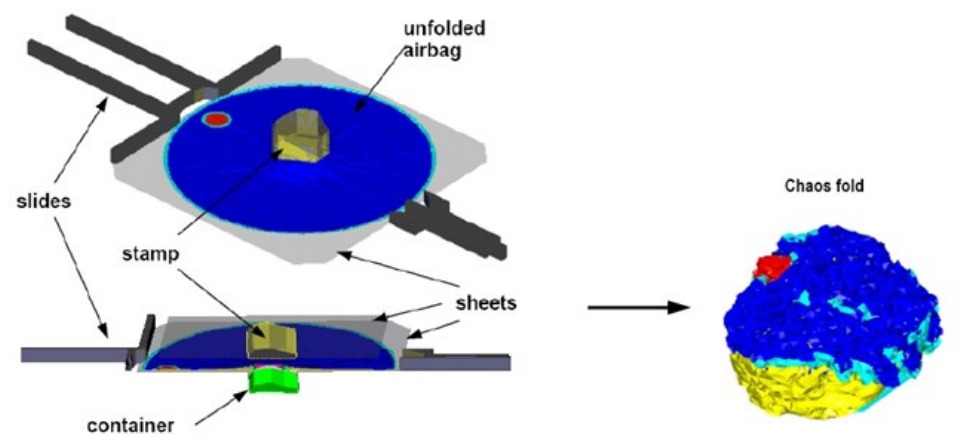

Figure 5.5: Simulation of folding as part of the manufacturing process [73].

Bosio and Mahangare [80] also analyzed the Origami folding method, the manufacturing process folding, and the mapping method by comparing them one another. The methods for modeling folding can be separated into five categories. Table 5.1 summarizes the advantages and disadvantages of each category:

a) Simulation of the flatten/folding process: This method uses initial pressure to flatten the 3D mesh and perform folding by moving nodes or splitting elements [71].

b) Mesh and fold the 2D surfaces: This method defines a folding line on a 2D meshed surface and the folding line is used for geometric transformations to create the folds. The type of folds such as thin fold, thick fold, roll fold, tuck fold and double tuck fold can be created through this method [71].

c) Mesh, deflate, and fold the 3D surfaces: A 3D meshed model is flattened by moving the nodes located at selected edges outward with appropriate scaling. 
Similarly to Method (a), the folds are created by using geometric transformations on the meshed folding lines [71].

d) Meshing after flattening (flattening and meshing): This method divides a $3 \mathrm{D}$ geometry of the inflatable into a set of $2 \mathrm{D}$ surfaces. The $2 \mathrm{D}$ surfaces are flattened and folded using simplifications in the geometry. The folded surfaces are then meshed, connected, and prepared for further simulations [71].

e) Flatten and folding simulation: This method directly simulates actual folding process of a meshed airbag. The flat airbag is meshed initially. Then, several auxiliary rigid planes are used to hold and fold a meshed flat airbag. Although this method is time consuming, it generates a realistic mesh and helps simulate the inflation process in a more realistic way [79].

Table 5.1: Summary of folding methods.

\begin{tabular}{|c|c|c|c|}
\hline & Folding Methods & Advantages & Disadvantages \\
\hline 1 & $\begin{array}{l}\text { Simulation of the } \\
\text { flatten/folding } \\
\text { process }\end{array}$ & $\begin{array}{l}\text { - Straight forward to } \\
\text { create a folded model } \\
\text { close to reality }\end{array}$ & $\begin{array}{l}\text { - Element penetration or } \\
\text { distortion may occur. } \\
\text { - Time consuming }\end{array}$ \\
\hline 2 & $\begin{array}{l}\text { Mesh and fold the } \\
\text { 2D surfaces }\end{array}$ & $\begin{array}{l}\text { - Sufficient for folding a } \\
\text { 2D flatten airbag. } \\
\text { - Easy to implement. }\end{array}$ & - Not suitable for 3D geometry. \\
\hline 3 & $\begin{array}{l}\text { Mesh, deflate, and } \\
\text { fold the 3D surfaces }\end{array}$ & $\begin{array}{l}\text { - Sufficient for folding a } \\
\text { 2D/3D airbag. }\end{array}$ & $\begin{array}{l}\text { - May lead to incorrect volume, } \\
\text { surface area, and shape. } \\
\text { - Time consuming }\end{array}$ \\
\hline 4 & $\begin{array}{l}\text { Meshing after } \\
\text { flattening }\end{array}$ & $\begin{array}{l}\text { - Sufficient for folding a } \\
\text { 2D/3D airbag. }\end{array}$ & $\begin{array}{l}\text { - May lead to incorrect volume, } \\
\text { surface area, and shape. } \\
\text { - Time consuming }\end{array}$ \\
\hline 5 & $\begin{array}{l}\text { Flatten and folding } \\
\text { simulation }\end{array}$ & $\begin{array}{l}\text { - Sufficient for folding a } \\
\text { 2D/3D airbag. }\end{array}$ & $\begin{array}{l}\text { Element penetration or } \\
\text { distortion may occur. } \\
\text { - Time consuming }\end{array}$ \\
\hline 6 & Origami Folding & $\begin{array}{l}\text { - Sufficient for folding a } \\
\text { 2D/3D airbag. }\end{array}$ & $\begin{array}{l}\text { - } \text { May lead to sharp edges } \\
\text { - Folding lines must be defined } \\
\text { initially } \\
\text { - Time consuming }\end{array}$ \\
\hline 7 & $\begin{array}{l}\text { IMM Mapped } \\
\text { Folding }\end{array}$ & $\begin{array}{l}\text { - Sufficient for folding a } \\
\text { 2D/3D airbag. } \\
\text { - Easy to implement }\end{array}$ & $\begin{array}{l}\text { - Element penetration or } \\
\text { distortion may occur. } \\
\text { - Time consuming }\end{array}$ \\
\hline
\end{tabular}


Unlike folding a thin automotive airbag, a novel approach is needed to handle the folding process of a gigantic inflatable structure with a non-negligible membrane thickness as the full-scale prototype modeled in this work. The novel approach proposed in this work is a hybrid folding methodology consisting in using boundary conditions to control the nodes and elements that initially are designed to be in the folding lines, adding new connector elements to the model in between the folding steps, and using rigid plates to fold the full-scale prototype according to an actual folding process implemented experimentally [58].

The model created to simulate the complete folding process of the full-scale prototype consists of flattening, grounding, node translating, assigning connectors at specific nodes, and using rigid plates to complete the folding steps. The details of folding process are presented in section 5.3.

\subsection{Mass Scaling Implementation}

As explained in section 2.9 of Chapter 2, the mass scaling is implemented in the model to reduce the computational cost without losing accuracy of the dynamic response. Most of the literature report using the method of trial and error in order to obtain the optimized mass scaling factor.

Jung [62] uses the mass scaling factors of zero, $20,50,100,150$, and 700 to perform a sheet metal forming simulation. Jung comments that the optimized mass scaling factor improves computational cost and does not affect solution reliability. However, the inertial effect should controlled properly. Pan et al. [63] implement mass scaling factors $(3.97,4.24,7.32$, and 9.14) in an explicit model of three point bending simulation. Although the typical difference percentile between kinetic energy and internal energy is required to be less than 10\% (see section 2.9), Pan et al.'s model with optimized mass scaling factor is able to obtain values of kinetic energy of less than $5 \%$ of the internal energy throughout most of the time period of the simulation [63]. Pan et al. [63] address the same concern that too much mass scaling could change the mass distribution which affects the dynamic behavior of the system as mentioned in section 2.9 . 
Liu [81] uses mass scaling factors of 100 and 1000 to improve the computational cost of a dynamic finite element modeling for the process of spin forming. Liu's numerical result shows a good agreement with the experiment data [81]. Wang et al. [82] scale up the membrane density by 1000 times to perform a simulation of nonlinear deformations of solar sail membranes using explicit analysis. Wang et al. [82] also mention the mass scaling technique is able to reduce the computational effort, but the excessive mass scaling can alter the accuracy in predicting wrinkling patterns.

For this work, and in order to find the appropriate mass scaling factor, a simulation has been done to validate the selection of mass scaling factor used for analyses of folding and placement, relaxation, and deployment of models of a full-scale prototype presented in Chapter 5, 6, and 7, respectively. A model of flatten and unfolded full-scale prototype is inflated by a uniform pressure with mass flow rate of air of $1.044 \mathrm{~kg} / \mathrm{sec}$ (for 5 time steps) and uses a dynamic relaxation process (a standard practice in inflatable structure simulation [80], see also Chapter 6 for further details). The selected mass scaling factors are zero, $10,100,386$, and 1000 and are applied directly to the density of the materials used in the model.

Figure 5.6 shows the plots of ratio difference $\left(\frac{E_{k}}{E_{i}}\right)$ in percentage between the kinetic energy $\left(E_{k}\right)$ and the internal energy $\left(E_{i}\right)$ of the model with different mass scaling factors. Table 5.2 presents the relationship between the mass scaling factor and computational cost. Model with zero mass scaling spends 82 minutes to complete 1 step time of calculation and yields $0.01381 \%$ of the difference kinetic-internal energy. On the other hand, the model with mass scaling factor of 386 produced a $0.9226 \%$ of the difference kinetic-internal energy by using 3 minutes per step time calculation. Model that scales the mass up to 1000 times does not converge due to element distortion by excessive mass scaling. Therefore, a mass scaling factor of 386 is selected to be used in simulations of folding, placement, relaxation, and deployment. 
Table 5.2: Computational cost corresponding to mass scaling factor.

\begin{tabular}{|l|c|c|c|c|c|}
\hline \multicolumn{1}{|c|}{$\begin{array}{c}\text { Sass } \\
\text { Scaling }\end{array}$} & MS =0 & MS = 10 & $\begin{array}{c}\text { MS }= \\
100\end{array}$ & MS = 386 & $\begin{array}{c}\text { MS }= \\
1000\end{array}$ \\
\hline $\begin{array}{l}\text { Average difference \% } \\
\text { between kinetic energy } \\
\text { and internal energy (\%) }\end{array}$ & 0.01381 & 0.05303 & 0.2577 & 0.9226 & $\begin{array}{c}\text { Does not } \\
\text { converge }\end{array}$ \\
\hline $\begin{array}{l}\text { Computational cost } \\
\text { (minutes) per step time }\end{array}$ & 82 & 30 & 10 & 3 & - \\
\hline
\end{tabular}

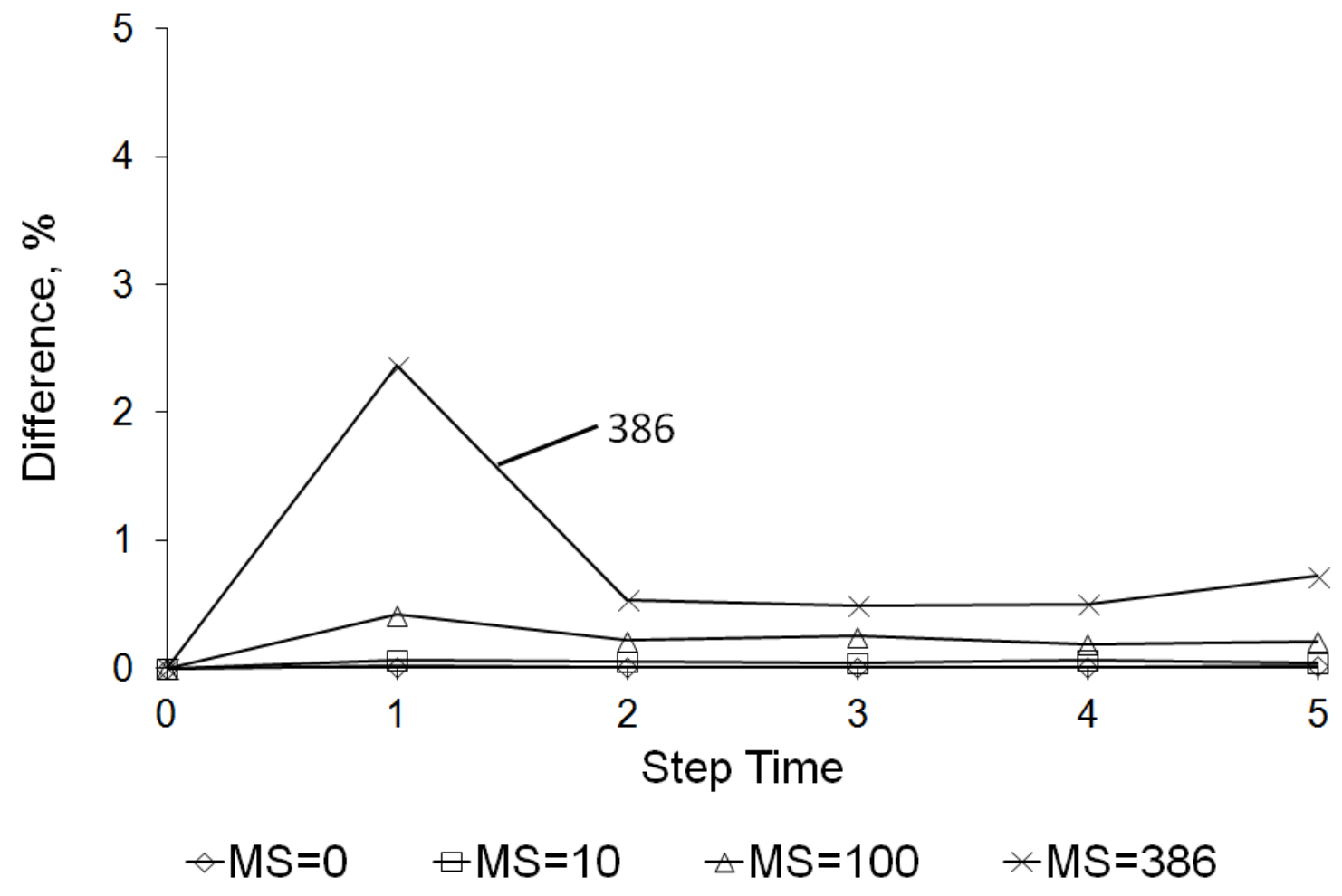

Figure 5.6: Mass scaling factor versus percentage difference between kinetic and internal energy

\subsection{Folding Plan}

In this study, Abaqus is used for the simulation of the folding process of the fullscale prototype. This folding process is developed as a set of the geometric transformations that replicates a folding sequence implemented experimentally in an actual prototype. The advantages of this novel approach are that the folding process is 
straight forward and allows to build a complete folded plug according to the actual folding process, connector elements are added into the model in between the folding steps, and the approach is able to avoid penetration and distortion of elements during the folding process.

The folding sequence developed in this work is shown in Figure 5.7. Figure 5.7(a) shows a top view of the flattened cylindrical region of the full-scale prototype. Figure 5.7(b) illustrates the folding sequence that follows the actual hand folding implemented experimentally. The folding process takes five steps after flattening in order to achieve the target shape. As seen in Figure 5.7(b), the folding sequence splits into two parts:

- Part 1: Line $C$ is translated and aligned with line $D$. These two lines are connected at 15 discrete points with fifteen connector elements (CONN3D2) evenly distributed along lines $C$ and $D$. The purpose of this initial step is to create an artificial wrinkle that "stores" material that will be released when the connector elements break during deployment process allowing more material to reach and cover intricate elements located in the upper portion of tunnel surface.

- Part 2: Once lines $C$ and $D$ are connected, the folding sequence continues with five rolling folds as illustrated in Figure $5.7(b)$. The folding by rolling demonstrated to be one of the simplest ways to fold an actual 2,000-pound fullscale prototype [58]. Two individual Abaqus Scripting input files were written to implement both Folding Part 1 and Folding Part 2. 


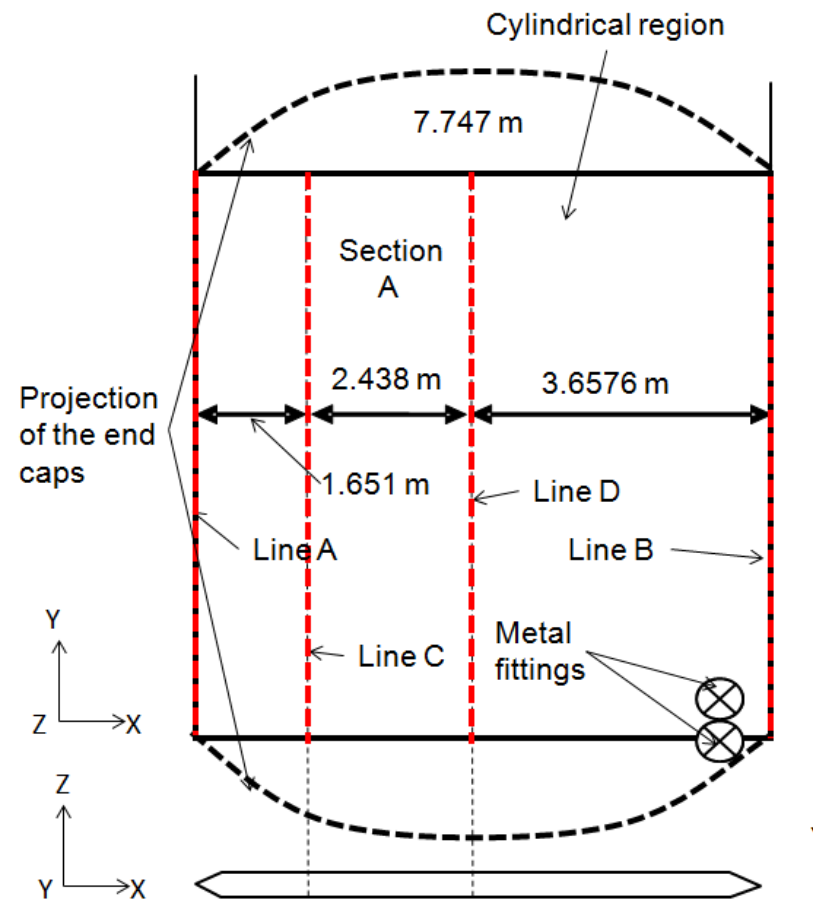

(a)

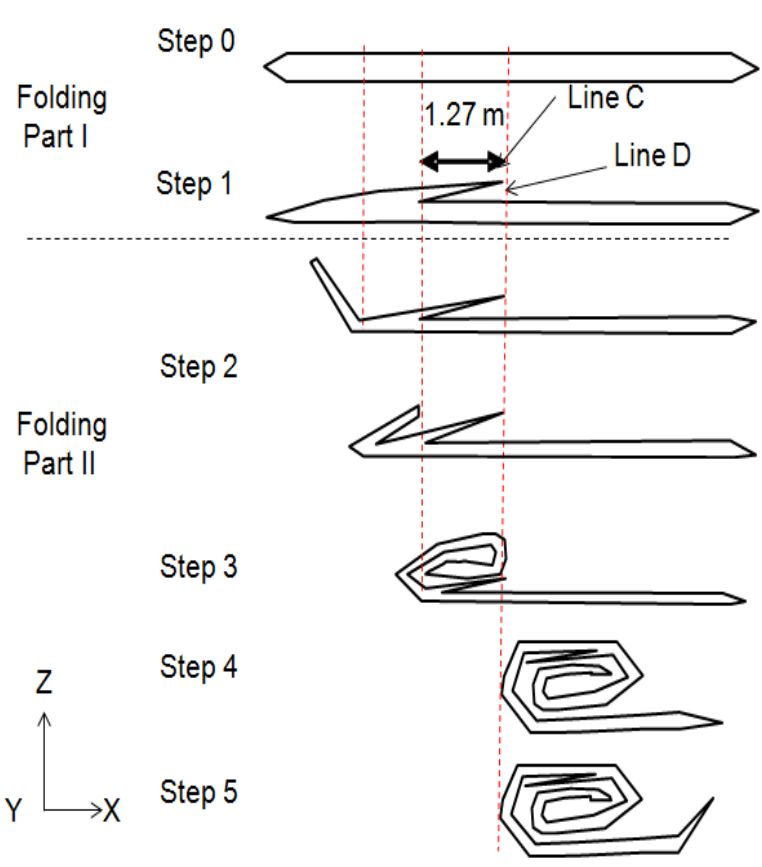

(b)

Figure 5.7: (a) Flattened cylindrical region. (b) Schematic of folding sequence after flattening.

The auxiliary parts created and presented in Chapter 4 are used for the folding process. They included the full-scale prototype meshed with membrane elements, the base plane meshed with rigid elements, and the rotational plates meshed with rigid elements as well. The parameters that are used to control the folding sequence included gravity force, position of folding lines, folding surfaces, axis of fold or roll, rolling angle, and translating distances.

The meshed parts were originally generated with Abaqus and renumbered with HyperMesh previous to the folding process. These parts were initially positioned in the global coordinate system defined in Abaqus/CAE and remained in the same orientation when writing into Abaqus Scripting. Friction coefficients of contact interaction properties between plug-base plane and self friction of the deflated plug were assumed to be 0.19 and 0.21 , respectively.

With all the parts in place, the folding process begins with flattening the full-scale prototype, which is initially in a unconstrained inflated condition (Figure 5.8). The plug's horizontal longitudinal axis $(z)$ is parallel to base plane which is assumed to be fully constrained. Line A and Line B located on the edges of the cylindrical portion. The 
deflation begins with a transverse horizontal translation of Line $A$ and $B$ along the $X$-axis. The base translates along $\mathrm{Y}$-axis by $2.286 \mathrm{~m}$ to reduce the kinetic energy and avoid penetration between elements (Figure 5.9). Gravity force is then applied on the plug along the -Y-axis and translates vertically the plug until it lies on the base plane (Figure $5.10)$.

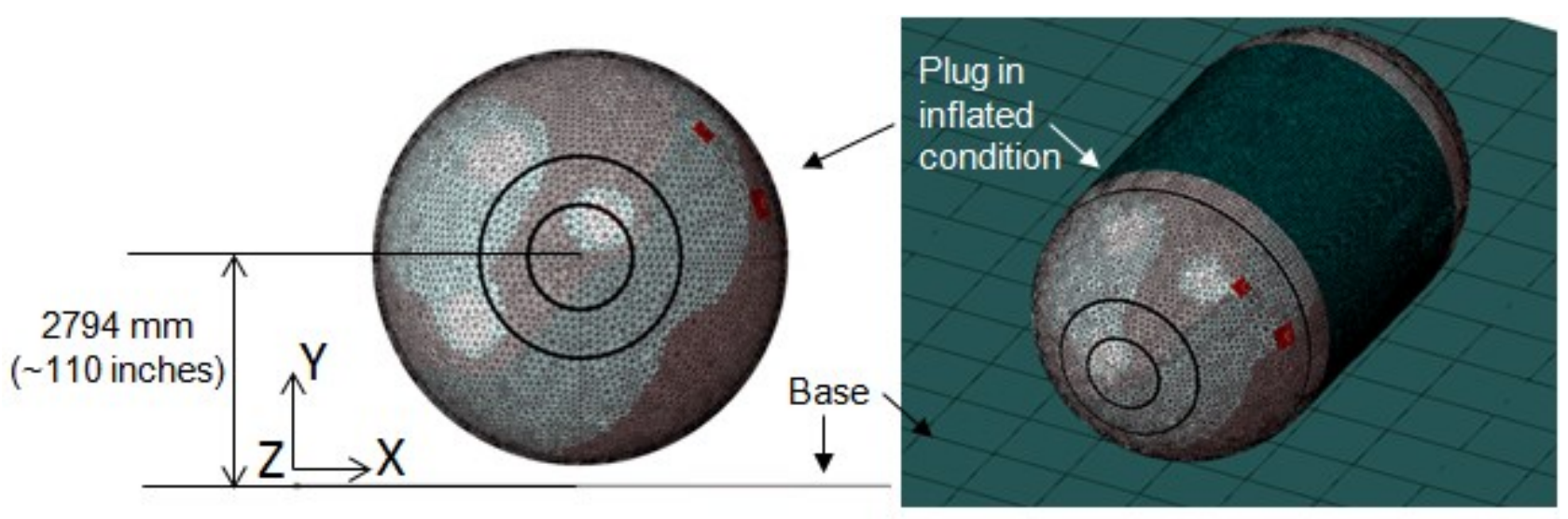

Figure 5.8: View of initial condition from XY-plane (left) and isometric (right).
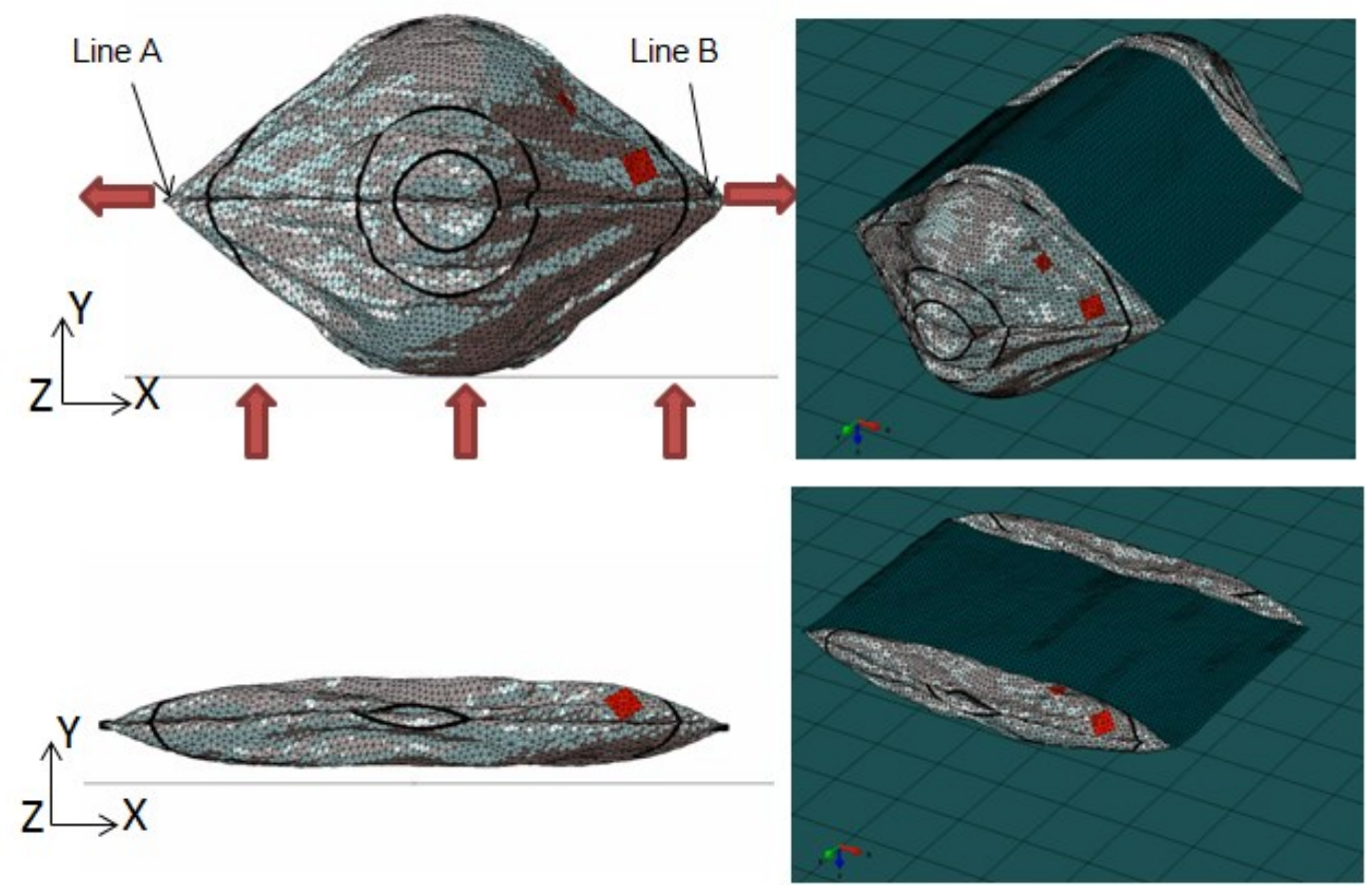

Figure 5.9: Translation of Line $A$ and Line $B$ along the $X$-axis. 


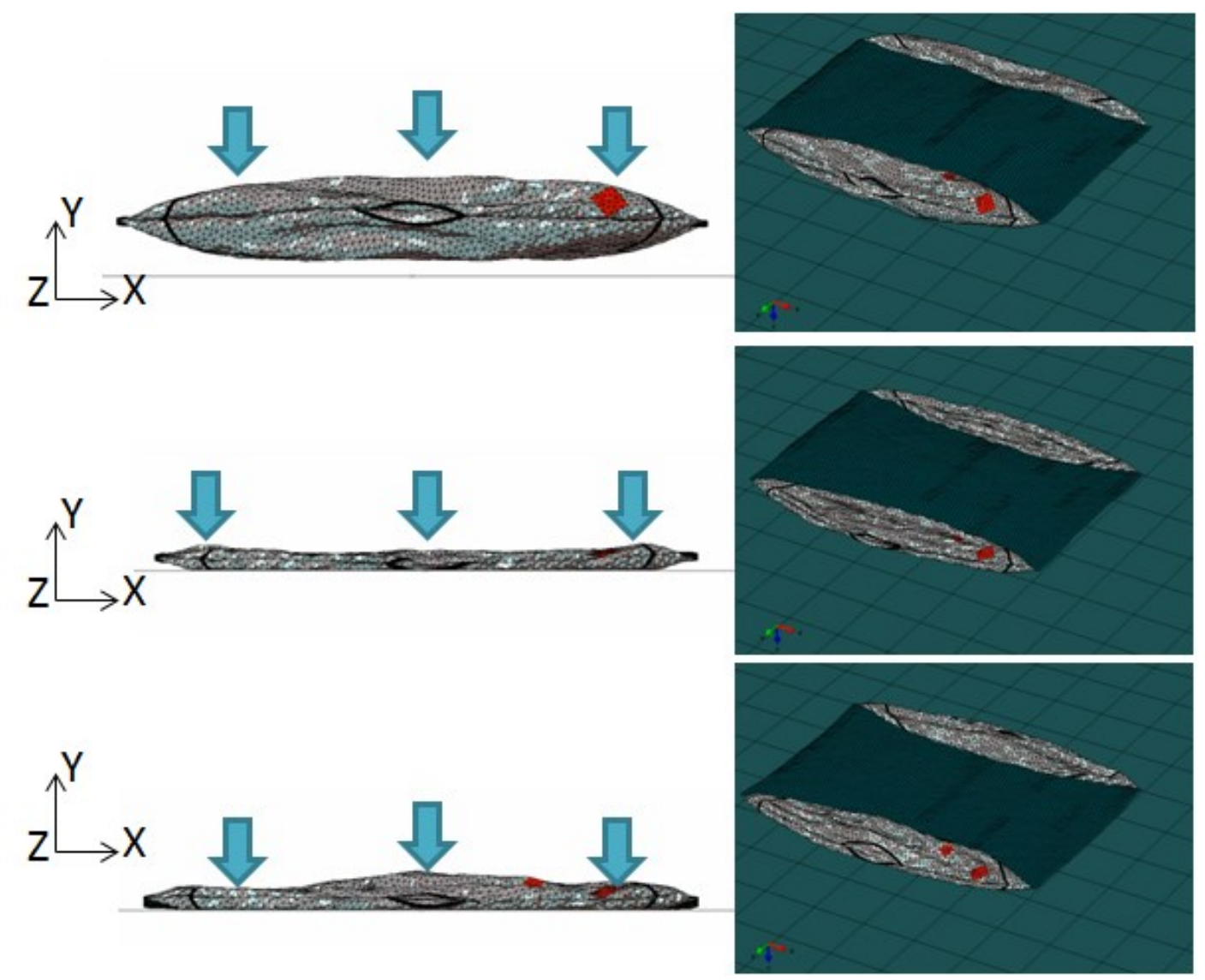

Figure 5.10: Gravity force acting on the deflated plug along the Y-axis.

Once the plug is completely deflated and sitting on the base plane, line C translates first along the $\mathrm{Y}$-axis $1.397 \mathrm{~m}$ (Figure 5.11). While the plug is continuously held down by gravity force until the translation is completed; then, line $\mathrm{C}$, again is translated along the X-axis $1.7272 \mathrm{~m}$ until it is aligned with line $\mathrm{D}$ as illustrated in Figures 5.12 and 5.13. The first part of the folding plan is considered complete at this stage. This first part of the folding plane was executed with Abaqus/CAE which was also used to export to HyperMesh the folding result shown in Figure 5.13. HyperMesh is then used to verify the final folded mesh geometry in order to detect and correct element penetration and intersections before proceeding to perform the second part of the folding plan. After verification of zero penetration and intersections, fifteen pairs of selected nodes from line $C$ and line $D$ are linked with connector elements through Abaqus Scripting. Initially, each connector element is assigned to have a tensile strength of $38308.99 \mathrm{~N} / \mathrm{m}$ (Appendix D). However, the tensile strength of simulation will 
be adjusted later to replicate the actual deployment behavior in further stages of the modeling.

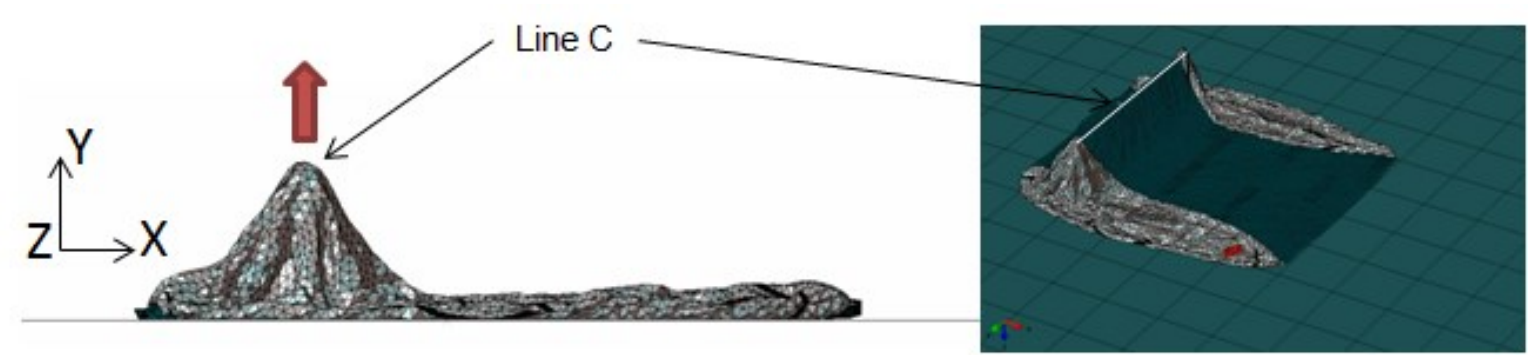

5.11: Line $C$ translates along the $Y$-axis.

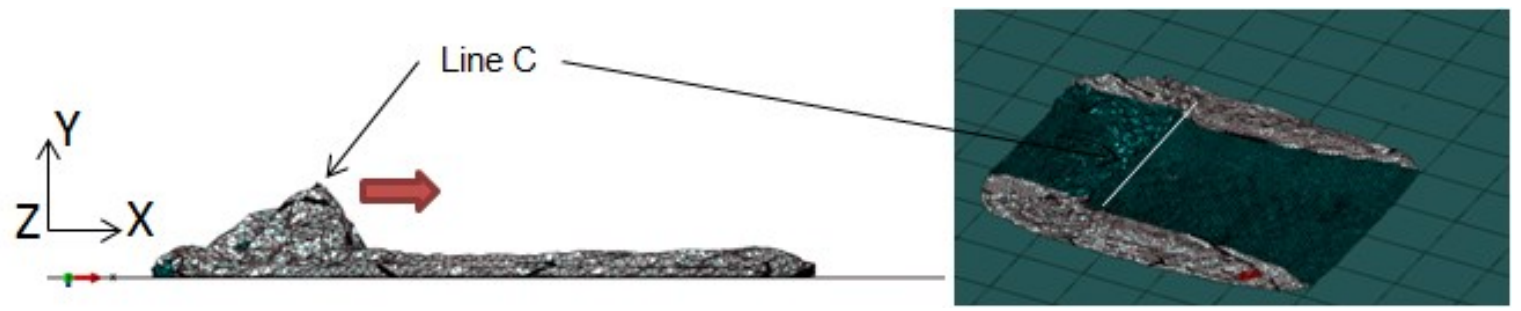

5.12: Line $C$ translates along the $X$-axis.

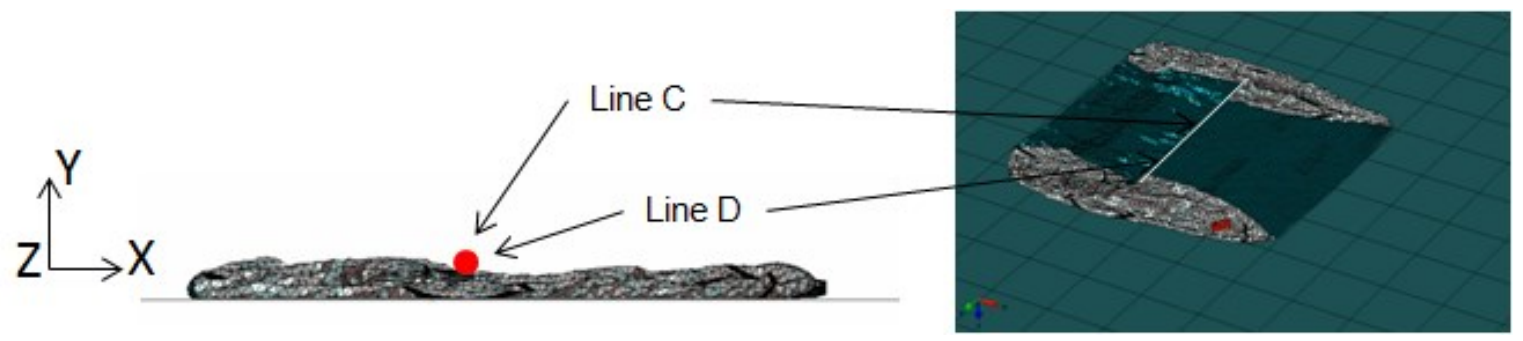

\subsection{3: Line $C$ aligns with Line $D$.}

Once the connector elements are installed, the second part of the folding process starts with the verified folded mesh geometry resting on the base plane. At this stage, four rigid plates are added to perform the folding operations (Figure 5.14). The rotational plates are placed along the Z-axis and slightly underneath the base plane. As mentioned previously, the friction coefficient between rotational plates and the deflated plug is assumed to be 0.19 . The contact interaction only applies between plug and the rigid bodies (base plane and rotational plates). However, there is no contact interaction assigned between the rigid bodies. The folding routine of each rotational plate consists of a single translation followed by a single rotation. 


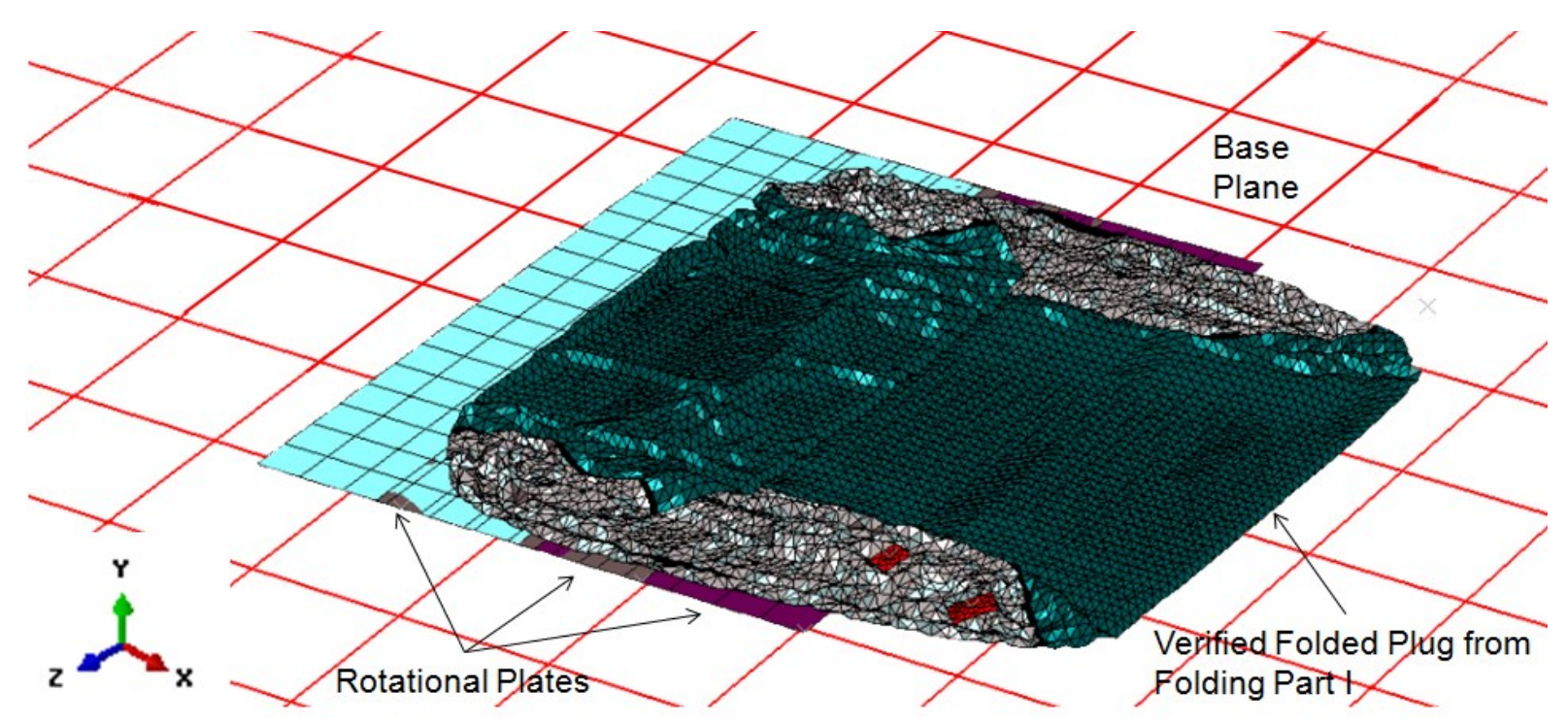

Figure 5.14: Initial condition for Folding Part II.

The first fold begins with the rotational plate \#1 translating along the $\mathrm{Y}$-axis 127 $\mathrm{mm}$ (5 inches) as shown in Figure 5.15, and then rotating clockwise $120^{\circ}$ about the zaxis as shown in Figure 5.16(a). When rotational plate \#1 finishes the rotation, the folded region falls free due to gravity force along the $\mathrm{Y}$-axis from the plate onto the surface of the plug as shown in Figure 5.16(b). Then, rotational plate \#2 translates $190.5 \mathrm{~mm}$ (7.5 inches) along the $\mathrm{Y}$-axis as shown in Figure 5.17 , and then rotates clockwise $135^{\circ}$ about the Z-axis as shown in Figure 5.18(a). Again, the gravity force helps to complete the third fold (Figure 5.18(b)).

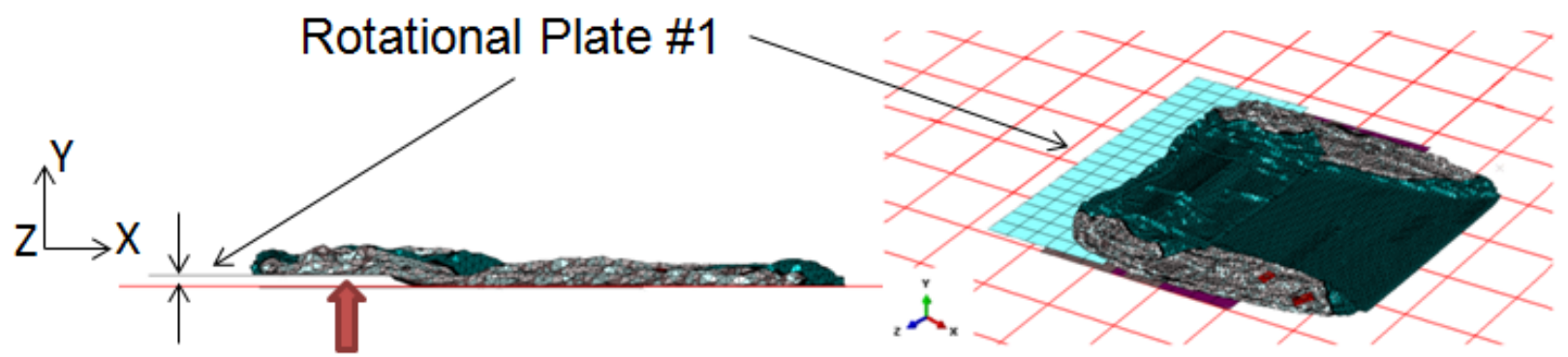

Figure 5.15: Rotational Plate \#1 translates along the Y-axis. 

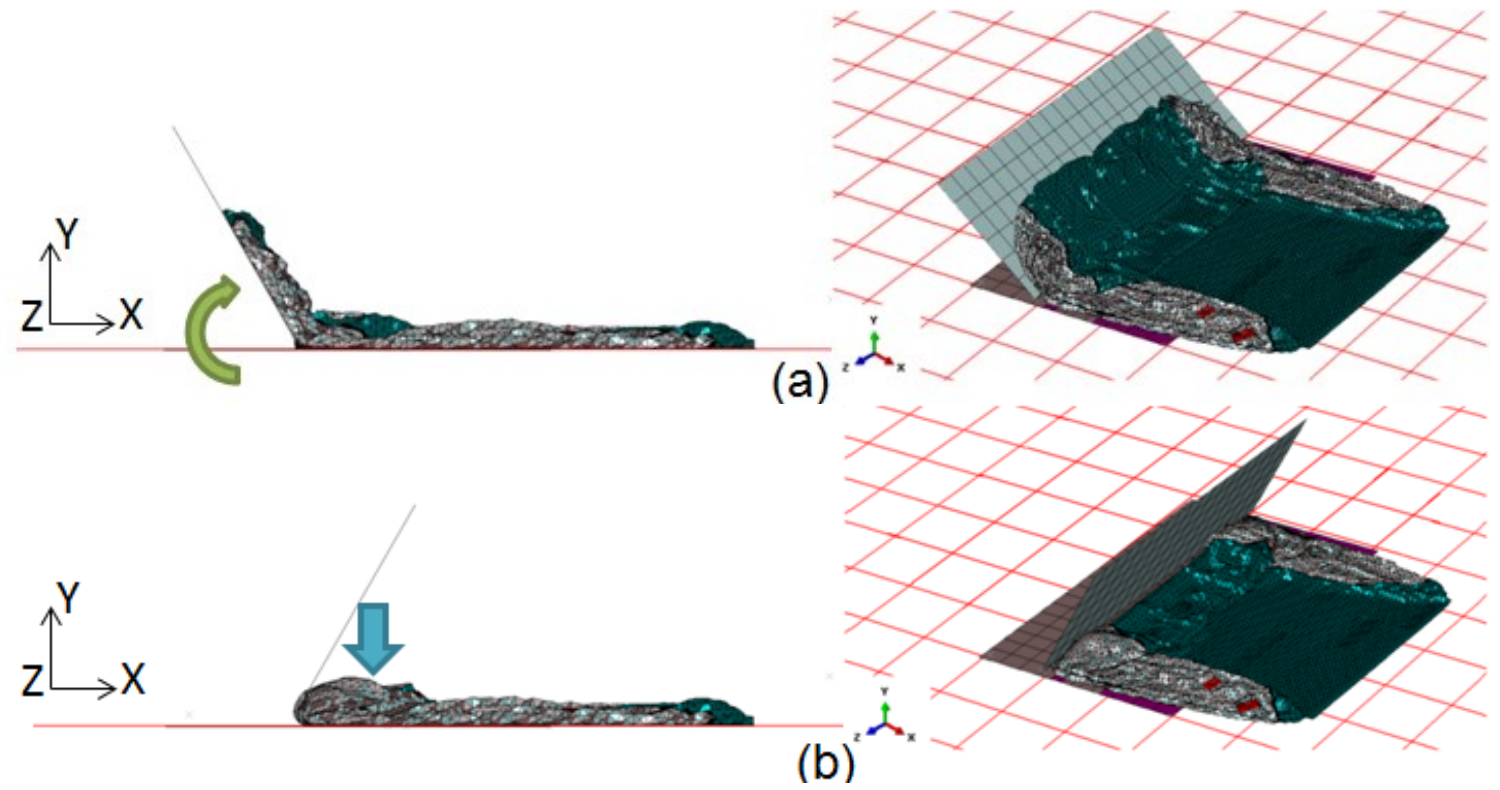

Figure 5.16: (a) Rotational Plate \#1 rotates clockwise $120^{\circ}$ about the Z-axis. (b) Gravity force acting to complete the fold.

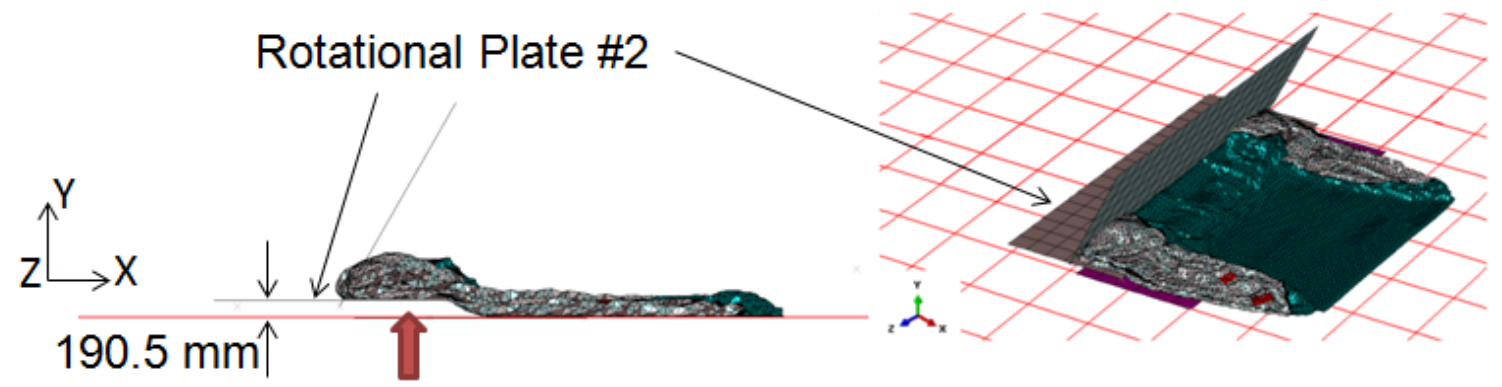

Figure 5.17: Rotational Plate \#2 translates along the Y-axis.
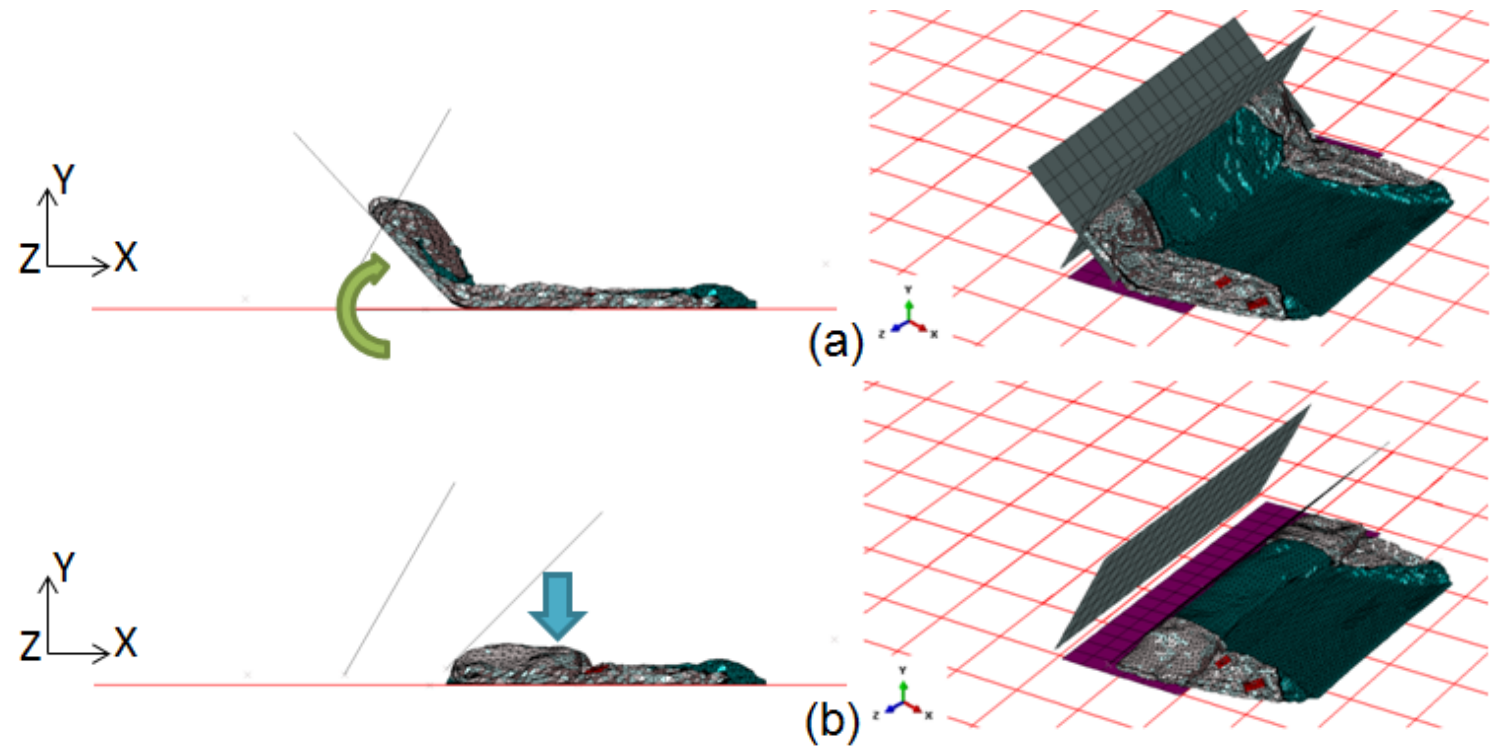

Figure 5.18: (a) Rotational Plate \#2 rotates clockwise $135^{\circ}$ about the Z-axis. (b) Completion of second folding by action of gravity force. 
The same folding routine applies to the fourth fold as the rotational plate \#3 translates $190.5 \mathrm{~mm}$ (7.5 inches) along the $\mathrm{Y}$-axis as shown in Figure 5.19, and then rotates clockwise $135^{\circ}$ about the Z-axis as illustrated in Figure 5.20. Gravity force completes the fourth fold. In order to make the placement process inside the storage area in the tunnel more convenient, a vertical translation is added on line B defined in Figure 5.7. At the end of the entire folding process, line $B$ translates $381 \mathrm{~mm}$ (15 inches) along the $Y$-axis for as shown in Figure 5.21. Line $B$ will be aligned and attached to a connecting line in the tunnel during the placement process described in Chapter 6.

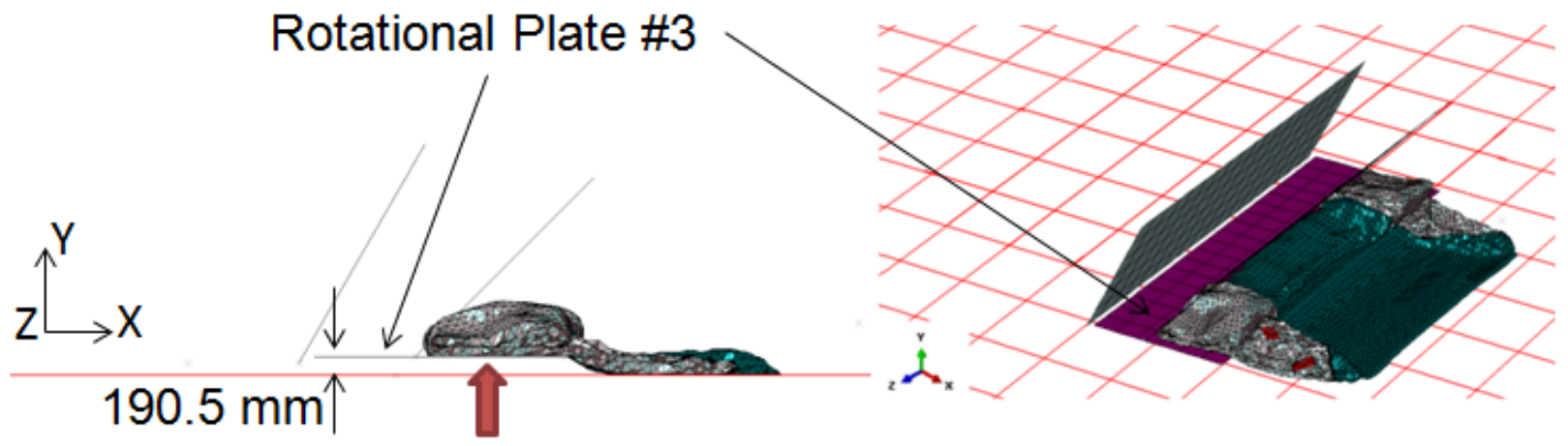

Figure 5.19: Rotational Plate \#3 translates along the Y-axis.

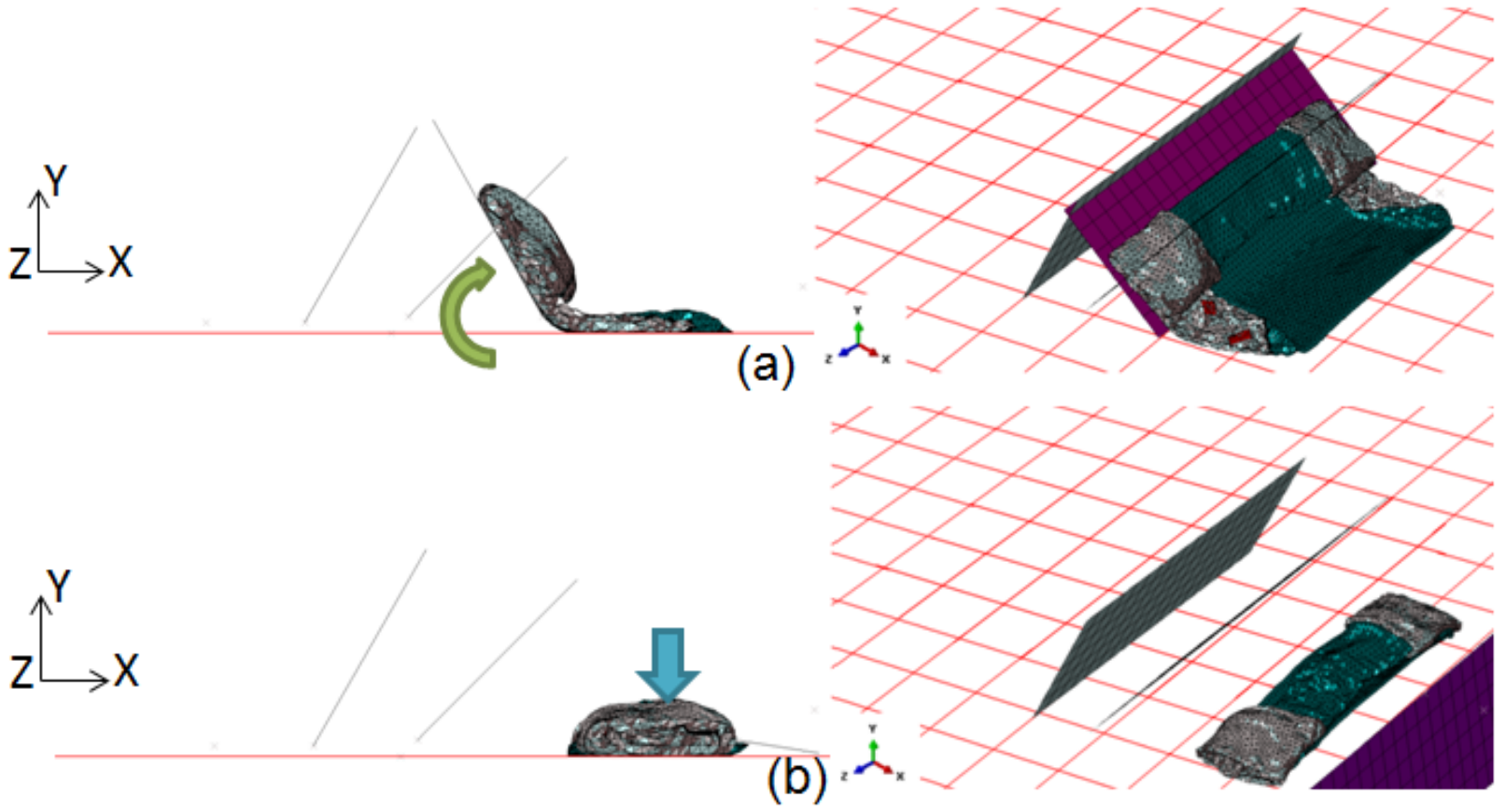

Figure 5.20: (a) Rotational Plate \#3 rotates clockwise $135^{\circ}$ about the Z-axis. (b) Completion of third folding by action of gravity force. 


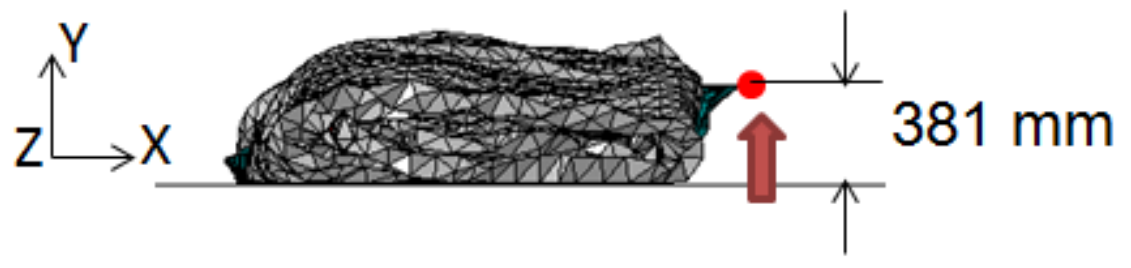

Figure 5.21: Line $B$ of plug translates along the $Y$-axis.

\subsection{Summary}

A folding process for a FE model of a full-scale prototype consisting of a combination of translation and rotation of rigid plates was implemented in Abaqus. The FE folding process replicates a sequence implemented experimentally. The verification of element penetration and intersection is a necessary process to detect and correct contact inconsistencies in order to create an error-free geometry before the implementation of the deployment process. The penetration and intersection feature of HyperMesh was used to systematize the inspection and correction of element distortions in order to eliminate errors and therefore prepare the resulting folded mesh for its placement inside the tunnel. 


\section{Full-Scale Prototype: Placement and Dynamic Relaxation}

\subsection{Introduction}

This chapter describes the process of placing the folded full-scale prototype into the storage area and the procedure for applying dynamic relaxation on the model. The folded plug as described in Chapter 5 , is placed in the storage area and held by a vertical rigid plane that represents an actual enclosure used to hold the plug within the storage area available in a typical tunnel section before deployment [58]. Figure 6.1 shows a schematic of the storage configuration. The rigid plane not only simulates the enclosure, which is activated during the deployment, but also maintains the folded fullscale prototype within the storage area during the application of the dynamic relaxation process.

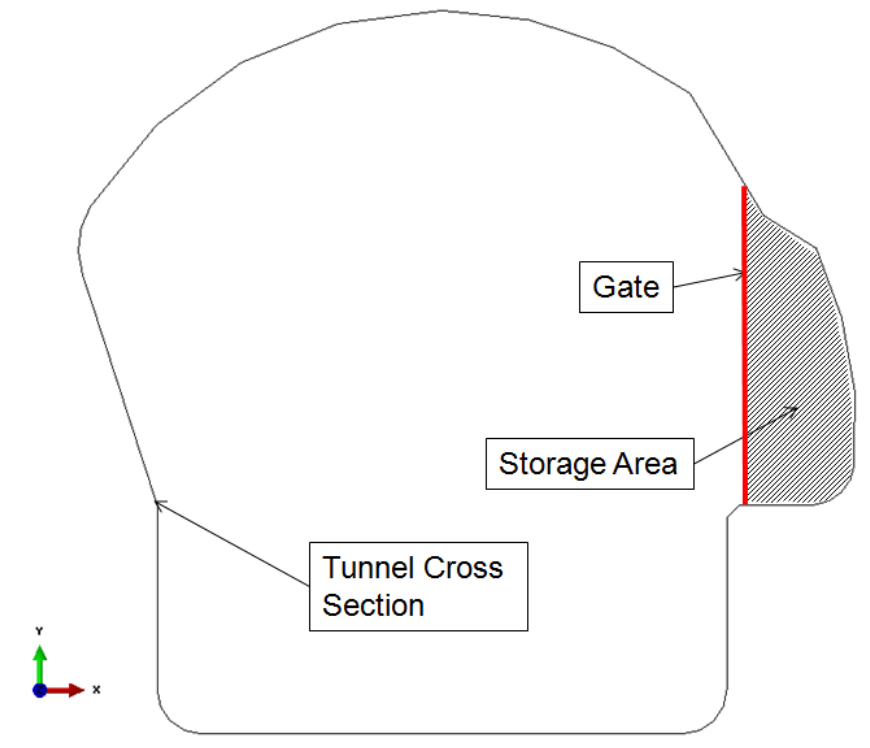

Figure 6.1: Location of Storage Area inside a Tunnel.

The main purpose of implementing the dynamic relaxation process into the simulation is to restore distorted elements back to their initial condition before doing the deployment simulation. The implementation of the dynamic relaxation process is a standard practice in airbag simulations [80]. Most of available commercial software execute the relaxation process through passing either node's or element's coordinates from an initial configuration to a reference configuration [113-115]. Section 6.3 describes this process in detail. 


\subsection{Placement}

The simulation of the placement process consists of a combination of a rigid body rotations and translations of the folded plug in order to position it in the storage area. Then, the folded plug is connected to the tunnel along a predefined horizontal line located inside the storage area that provides alignment to the plug during the deployment and inflation process. The placement is completed by further translation originated by a horizontal gravity force. Figure 6.2 shows the initial orientation of folded plug and the tunnel cross section.

The folded plug is rotated counterclockwise $45^{\circ}$ about the Z-axis at Line B that defined in Figure 6.2(a) and translated horizontally $-1041 \mathrm{~mm}$ and vertically $-254 \mathrm{~mm}$ along the global $\mathrm{X}$-axis and $\mathrm{Y}$-axis, respectively. Figure 6.2(b) shows the orientation of the plug after initial rotation and translation.

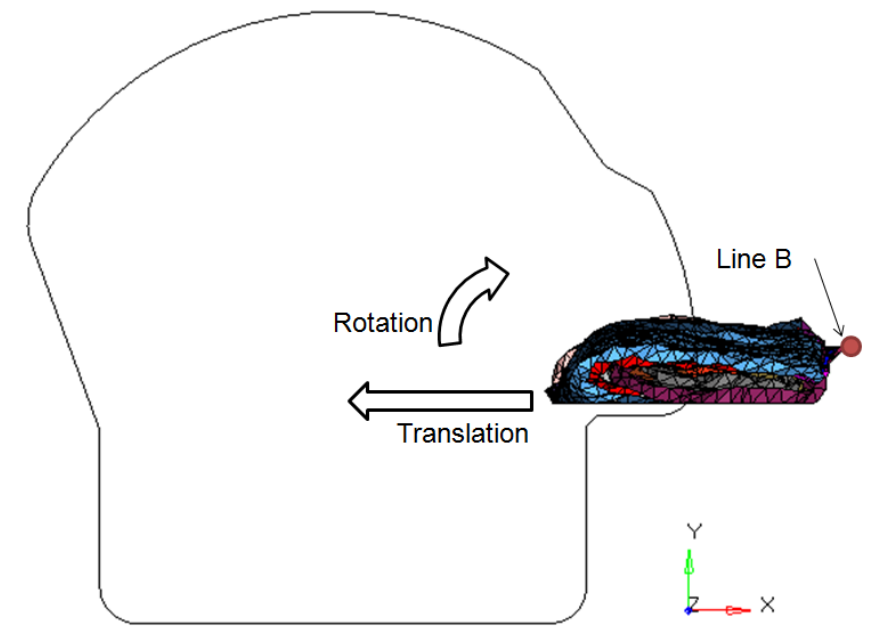

(a)

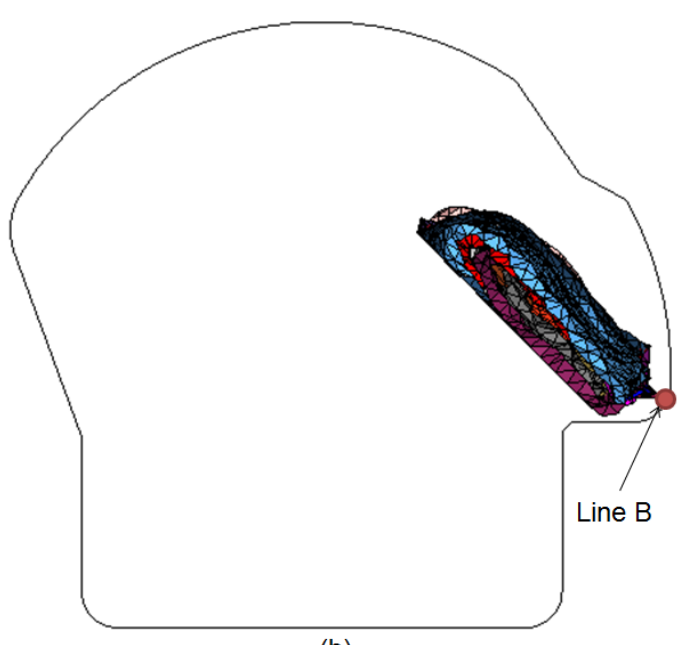

(b)

Figure 6.2: (a) Initial condition for placement; (b) Orientation of folded plug after rotation and translation.

The simulation of placement process continues with the connection of the folded plug to a selected line inside the storage area. Twenty two nodes from Line B were selected and aligned individually along the $\mathrm{X}$-axis and $\mathrm{Y}$-axis to match nodes located along line $B$ in the tunnel cross section. Figure 6.3 shows the twenty two selected nodes on line $B$ before the beginning of the placement simulation.

When the placement simulation started, the nodes were aligned to the specific coordinates initially along the global $\mathrm{X}$-axis and $\mathrm{Y}$-axis only. Then, the folded plug was 
shoved into the storage area by normal force along the X-axis. Figure 6.4 displays the final position of folded full-scale prototype and connecting nodes before the application of the dynamic relaxation process.

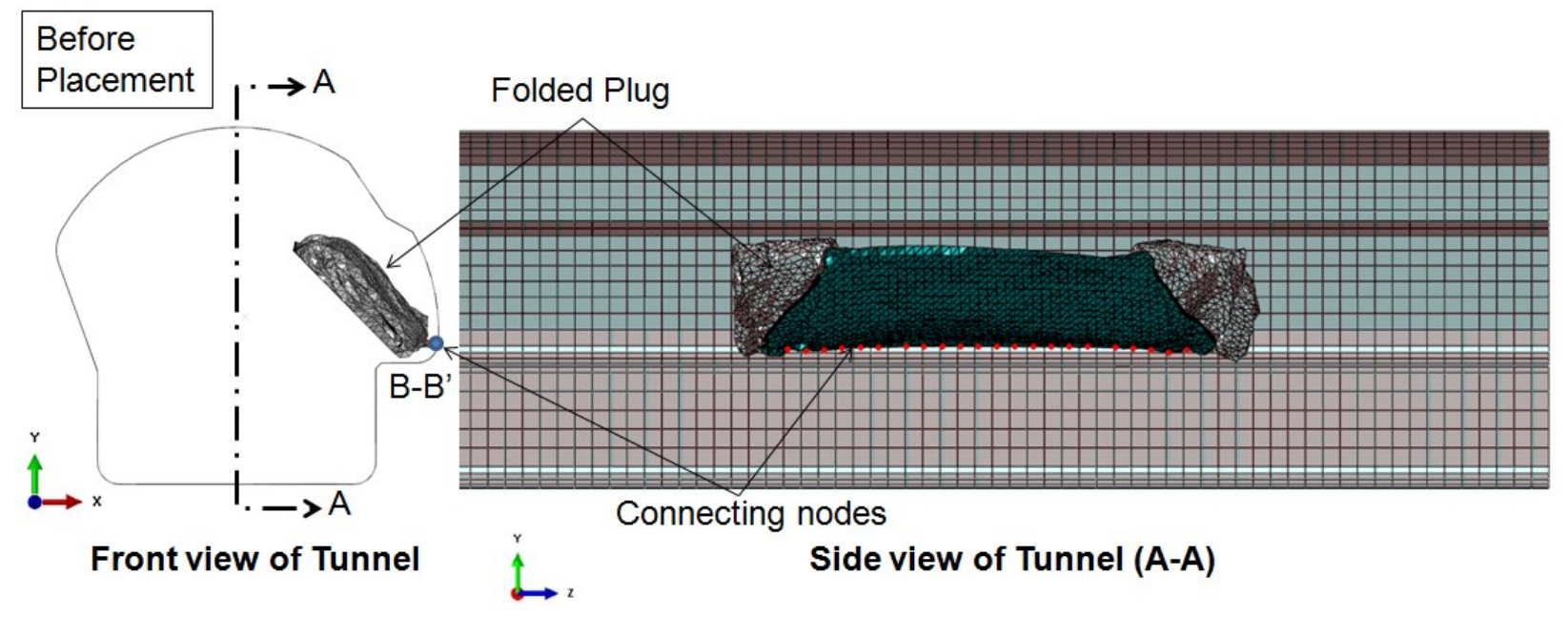

Figure 6.3: Connecting nodes of Line B.

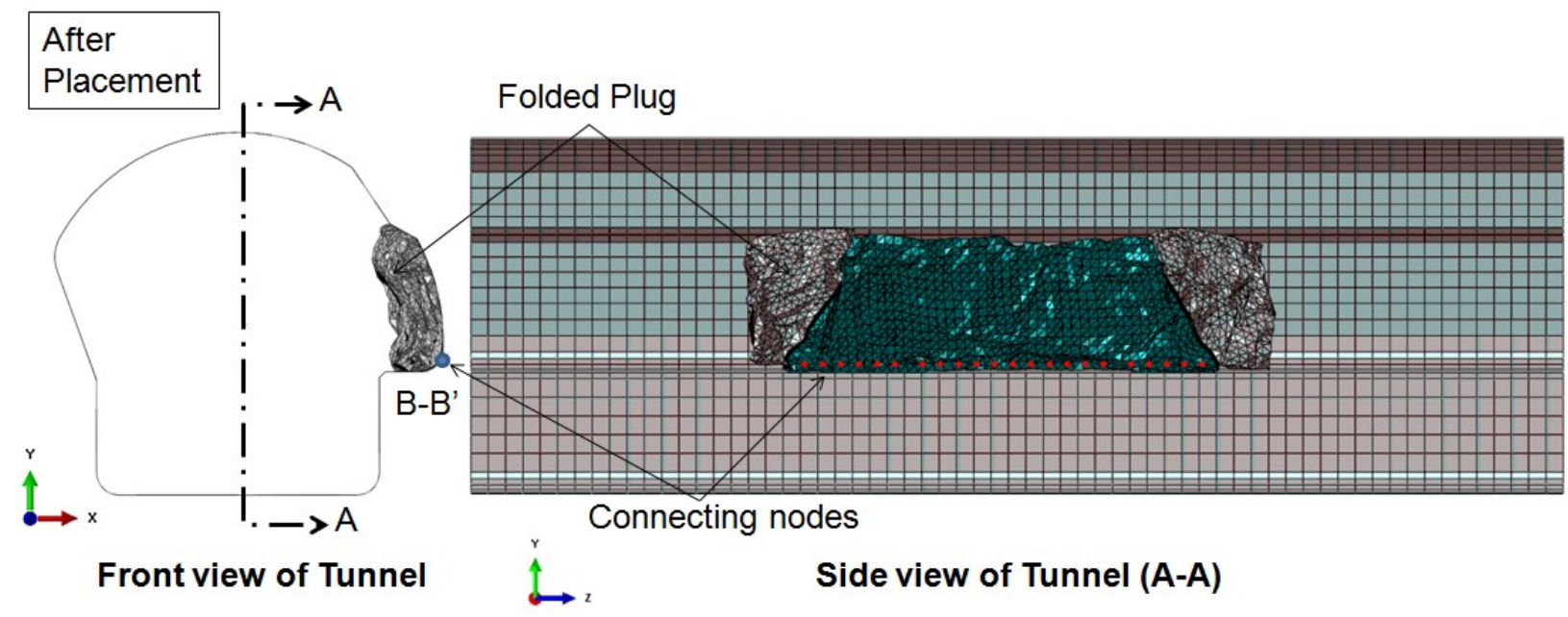

Figure 6.4: Connecting nodes after placement simulation.

\subsection{Dynamic Relaxation Method}

\subsubsection{Introduction}

Dynamic relaxation is a numerical procedure that uses a simple vector iteration method to achieve the static equilibrium state by minimizing the energy [89]. The computational cost is reduced because the tangential stiffness matrix of the structure is not assembled when the dynamic relaxation performs iterative calculations during the 
stabilizing process [89]. The stabilizing process of an unstable structure consists in introducing initial pre-stresses to determine the member rigidities of potential unstable structures and verify if they are able to resist external loads effectively.

Typically, a pneumatic structure is considered an unstable structure and requires an initial pressure or pre-stress in order to be used as structural member to carry external loads [89]. Therefore, dynamic relaxation is a necessary process for airbag simulations [80] because it minimizes the kinetic energy of a linear or a non-linear model during the area restoration of the pneumatic structure through the initial metric method [75], typically caused by the folding process, to its initial area [71-115]. Lee et al. [72] used relaxation to stabilize the internal energy of the folded airbag in order to reach a stable condition at later stages of the simulation.

In the remainder of this section, sub-section 6.3.2 summaries the history of dynamic relaxation development and sub-section 6.3.3 presents the basic theoretical formulation that supports the relaxation process. Section 6.3.4 outlines the implementation of the the relaxation in Abaqus and relaxation scripting code that can be used to define the material orientation of a complicated pre-folded model [71-115].

\subsubsection{Brief History of Dynamic Relaxation}

Otter and Day (1960 and 1965) [84-88] developed the a numerical method called Dynamic Relaxation to handle form-finding and load analysis of non-linear structures. According to Underwood (1983) [83] the dynamic relaxation provides a static solution to a dynamic transient analysis model. Welsh [96] and Cassell [97] improved the dynamic relaxation method by introducing a fictitious mass in late 1960 .

Rushton (1968) [98] was the first to apply dynamic relaxation method into a nonlinear problem. Wood [99] compared and discussed about dynamic relaxation method with other iterative methods. The dynamic relaxation method has been extensively implemented [100-106] and improved [107-112] since 1970. Underwood (1983) [83] summaries the history of dynamic relaxation development very well in his paper. 


\subsubsection{Formulation}

The basic equations of dynamic relaxation can be written from the discrete equation of motion [83-116] as shown below,

$M a+C v+K u=R$

where $M$ is the mass matrix, $C$ is the damping matrix, $K$ is the stiffness matrix, $R$ is the vector of nodal equivalent residual forces, $a$ is the vector of nodal accelerations, $v$ is the nodal velocities, and $u$ is the vector of nodal displacements. The nodal residual force can be expressed as,

$R=F-T(\sigma)$

where $F$ is the vector external loads and $T$ is the vector of equivalent nodal force as a function of membrane stress, $\sigma$. The nodal residual force also can be rewritten by introducing the viscous damping to control the element distortion without considering the elastic stiffness and external loading [83-116],

$R=M a$

$R=-T(\sigma)-C v$

Using a central finite difference form [117] to solve the equation of motion [93],

$a_{n}=\frac{1}{\Delta t^{2}}\left(u_{n+1}-2 u_{n}+u_{n-1}\right)$

$v_{n}=\frac{1}{2 \Delta t}\left(u_{n+1}-u_{n-1}\right)$

and the diagonal mass matrix of the equations of motion can be solved for a position at step $(n+1)$ [93],

$x_{n+1}^{i}=x_{n}^{i}+u_{n+1}^{i}$

$u_{n+1}^{i}=2 u_{n}^{i}-u_{n-1}^{i}+\frac{R_{n}^{i}}{m_{i i}} \Delta t^{2}$

where $x_{n+1}^{i}$ is the position of degree of freedom of $(i), m_{i i}$ is the diagonal mass, and $R_{n}^{i}$ is the residual force that can be obtained from equation 6.3.

Barnes [91] explained that the iterative computational procedure of dynamic relaxation can be summarized as follows:

1. Formulate all the residual force $(R)$ of the nodes, all the nodal velocities $(v)$, and kinetic energy (from previous simulation if there is any) to zero. 
2. Formulate the residuals $(R)$ equal to all the applied load, $F-T(\sigma)$, components.

3. Calculate the forces from the current element stress and add the calculated results to the residuals (equation 6.3).

4. All the fixed residuals and partial constrained nodes are to be reset to zero. This resetting restricts both the residuals and partial constrained nodes moving in the constrained direction.

5. Calculate all nodal velocities components and update the geometry (node coordinates) using Step 2 and 3. Calculate the current kinetic energy (KE) of entire system at $n+1$.

6. If current kinetic energy is lower than previous $n+1$, return to step 3 .

7. If current kinetic energy is higher than previous $n+1$, apply small corrections to all node coordinates corresponding to the true kinetic energy peak time (Figure 6.5).

8. Repeat the calculation process from step 1 until the structure is in the static equilibrium (low kinetic energy and nodal residuals).

Steps 3-6 are performed at each iteration and step 1-8 are executed between energy peaks [90-91]. In Figure 6.5, the energy peak $A$ is an early kinetic energy that corresponds to high frequency modes due to large unbalance forces in the boundary [90-91]. The energy peak B corresponds to the overall structural form at low frequency modes (which are normal to the changing surface after those modes in energy peak $A$ have been damped out substantially [90-91]). The energy peak $C$ occurs rapidly and corresponds to a slight in plane motion to complete the convergence [90-91].

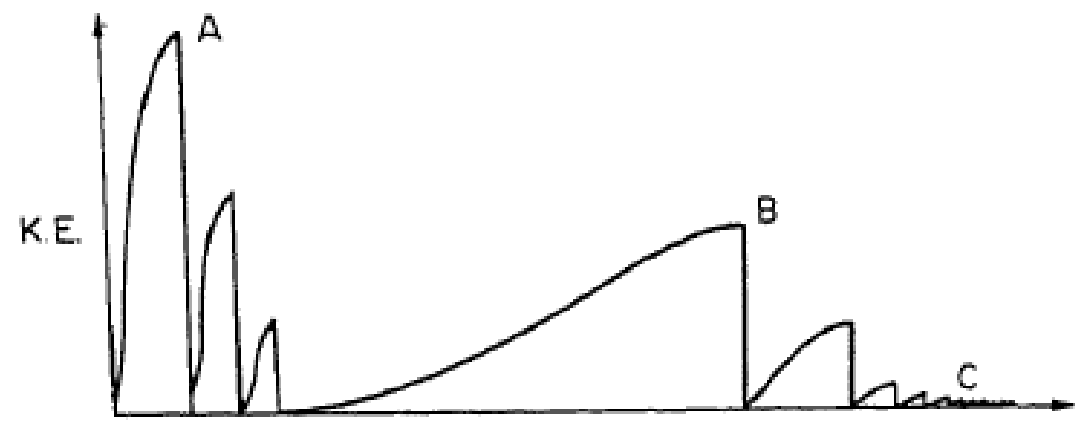

Figure 6.5: Kinetic Energy Plot Pattern of Dynamic Relaxation [90-91]. 


\subsubsection{Implementation}

Abaqus/Explicit provides two options for users to execute the relaxation process [4950] on membrane elements only.

I. Defining the reference mesh through element numbers and coordinates of all the element's nodes (Input File Keyword: TYPE=REF COORDINATE).

II. Defining the reference mesh through node numbers and coordinates of the nodes (Input File Keyword: TYPE=NODE REF COORDINATE).

For the first option, the keyword of TYPE=REF COORDINATE specifies the reference mesh of all initial metric for membrane elements through indicating the element numbers and the coordinates of all the element's nodes. All elements are assumed located on one plane, therefore it's easy to define material orientation under this option and is more general in use.

On the other hand, for the second option, the keyword TYPE=NODE REF COORDINATE is used to specify the reference mesh of all initial metric for membrane elements through indicating node numbers and the coordinates of each node. The user needs to define the specific material orientation (for instance, direction) under this keyword and this option usually applies when the user wants to assign a specific material orientation on a fully folded geometry in which the elements have laid in different planes [115].

These options are useful to reconfigure or remove the wrinkles that occur during the folding process. The reference configuration may be suitable to be defined from an unstressed mesh model because the corresponding folded mesh may need the initial state to define the folded state [115]. Based on the material definition, the nonzero stresses and strains may occur if the reference configuration was defined differently from the initial configuration. The initial stress and strain of the model are calculated to constitute the governing deformation from the reference to the initial configuration [115]. However, the initial stress or strain conditions of a specific element are neglected if the reference mesh is indicated for that specific element [115]. Also, Abaqus/Explicit allows users to control the amplitude of applying the load the stresses/forces gradually and smoothly by assigning the value of scale factor [115]. 


\subsection{Evaluation of Relaxation on Unconstrained Folded Plug}

A simple evaluation has been conducted to show the importance and necessity of applying the dynamic relaxation process to the model during deployment. The folded full-scale prototype shown in Figure 5.21 was used to perform an evaluation under unconstrained conditions. The test consisted of, first constraining one edge of the folded plug in a way that the nodes on line $B$ of the folded plug were restrained from any translation but free to rotate (i.e. 3 translation DOF $=0$ and 3 rotational DOF $\neq 0$ ); then, the folded plug was inflated with mass flow rate of $20 \mathrm{~m}^{3} / \mathrm{sec}$ until its initial volume $\left(151.7873 \mathrm{~m}^{3}\right)$ was reached at the ninth second (Figure 6.6). Figure 6.6 illustrates the results of both models with and without dynamic relaxation. Both models reached the stable volume at approximately the ninth second; however, the model without relaxation $(\boxminus)$ only reached $124.1695 \mathrm{~m}^{3}$ resulting in a loss of volume of approximately $18 \%$ with respect to the initial volume of $150 \mathrm{~m}^{3}$. The model that included relaxation reached the initial condition volume. Furthermore, the model that included dynamic relaxation did not show distorted elements as illustrated in Figure 6.7. The model without relaxation remained distorted at the moment of reaching its maximum volume as illustrated in Figure 6.8. This was considered an unacceptable result and demonstrated the importance of including dynamic relaxation to restore the original shape and volume during the inflation.

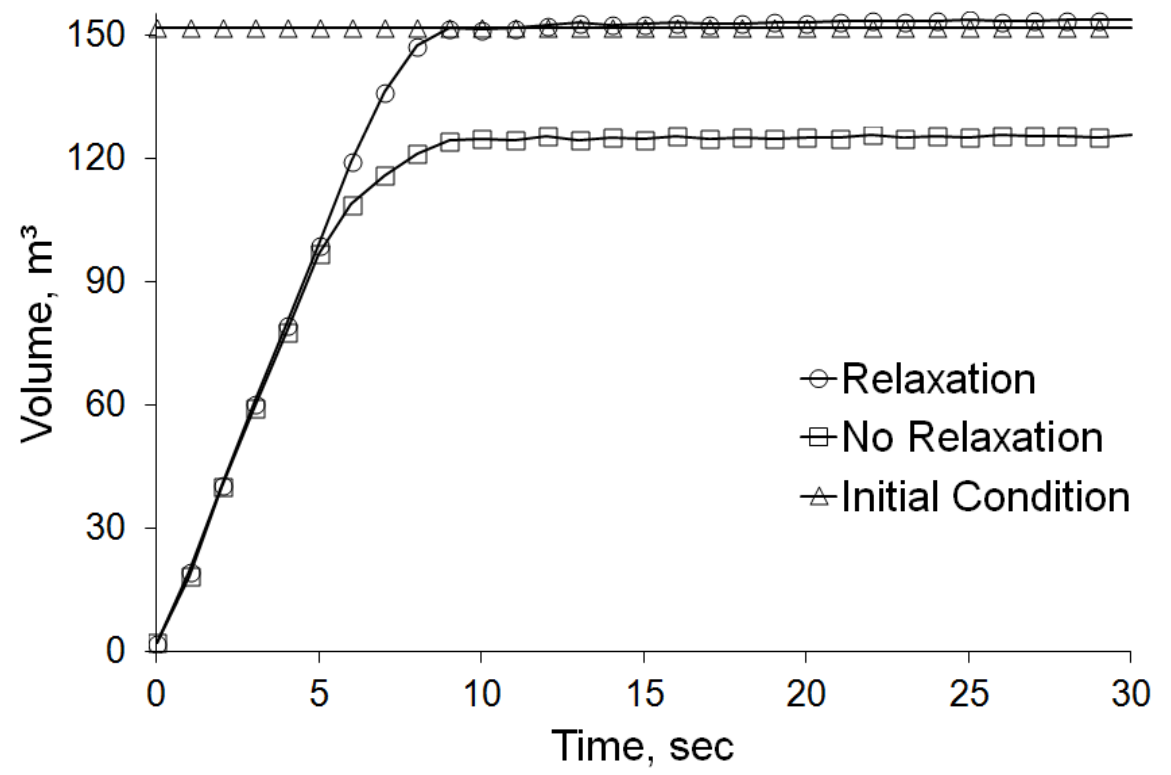

Figure 6.6: Evaluation of influence of relaxation through volume comparison. 

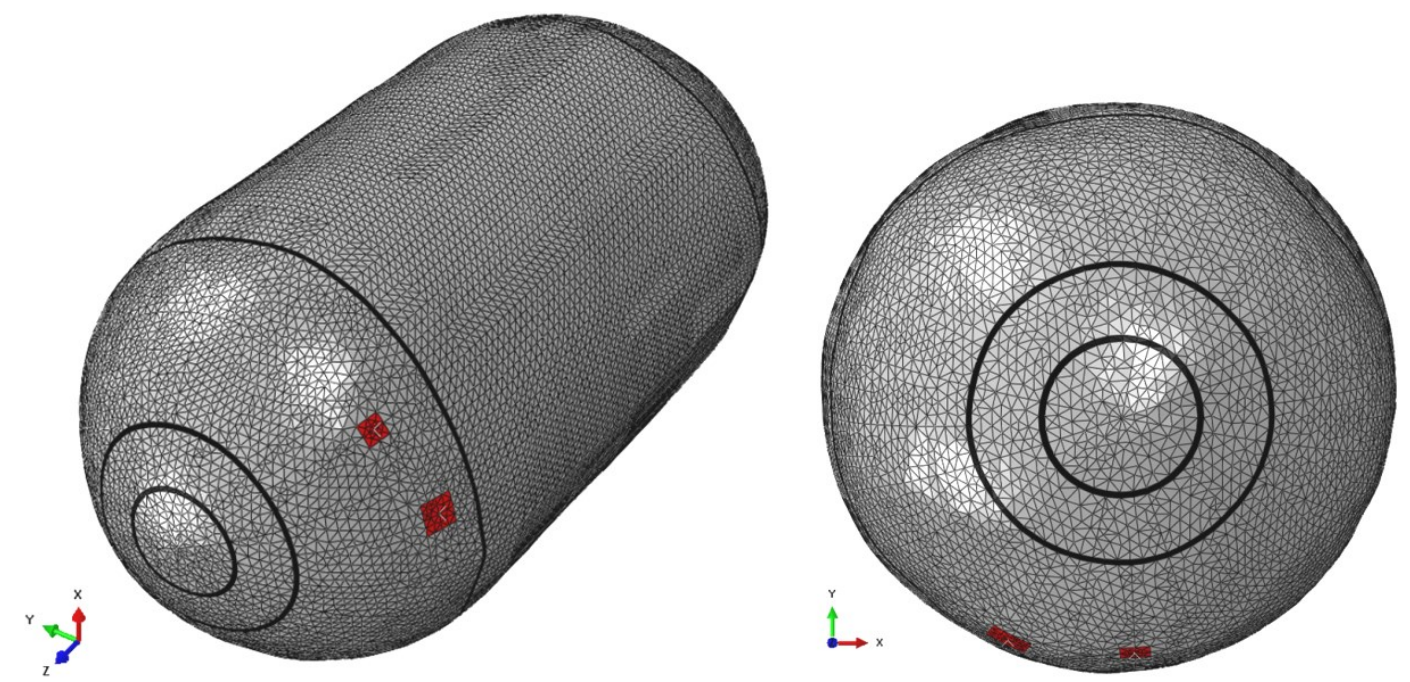

Figure 6.7: Model with Dynamic Relaxation @ $\mathrm{t}=25 \mathrm{sec}$ in Figure 6.6. Deformation Scale Factor $=1.0$.
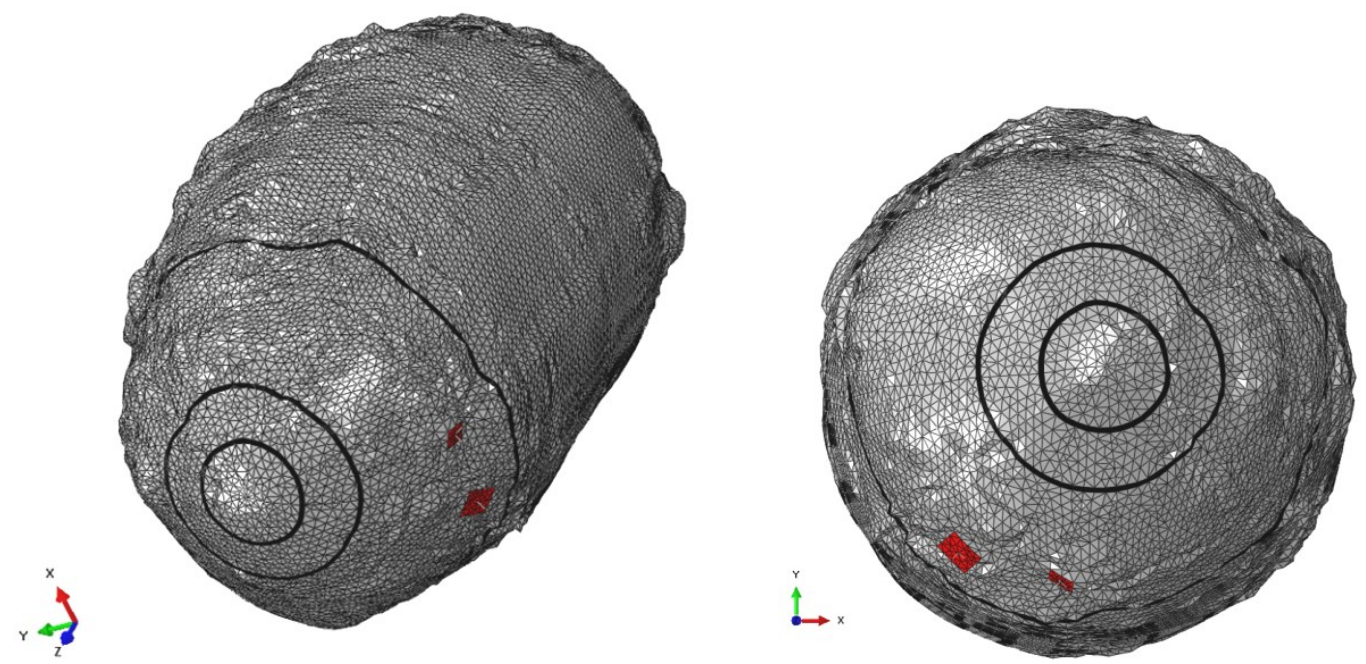

Figure 6.8: Model without Dynamic Relaxation @ $\mathrm{t}=25 \mathrm{sec}$ in Figure 6.6. Deformation Scale Factor $=1.0$.

\subsection{Relaxation Process on Folded Plug in Storage Position}

A rigid plane was created to represent the gate that encloses the storage area before the relaxation begins. In this way, the folded plug then can be placed freely within the storage area without unfolding during relaxation process. The keyword TYPE=REF COORDINATE was chosen to be applied in the model since the material 
orientation was previously defined in the material properties included in the scripting file. In the simulation, the rigid plane pushes and closes the storage area when relaxation simulation commences as shown in Figure 6.9 and Figure 6.10. Figure 6.11 shows the result of the relaxation simulation after it was completed.

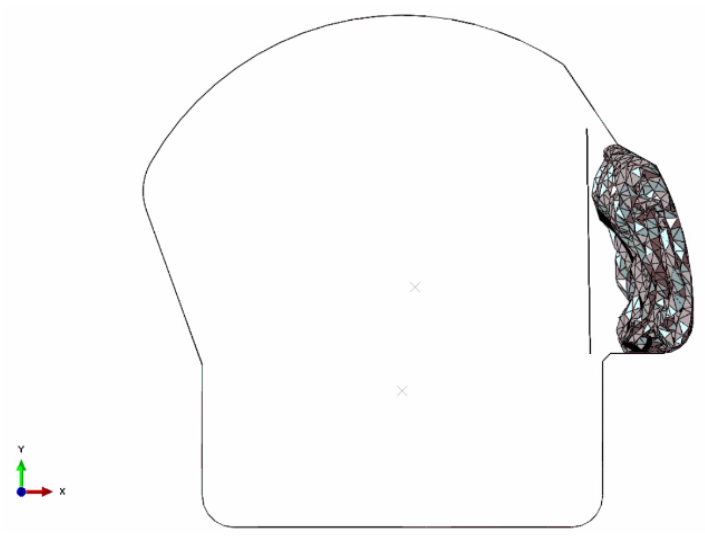

Figure 6.9: Rigid plane before closing the storage area.

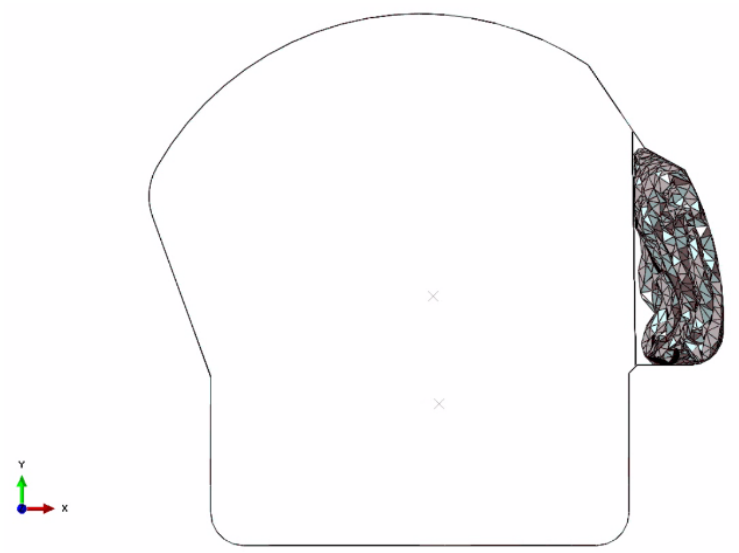

Figure 6.10: Rigid plane after closing the storage area.

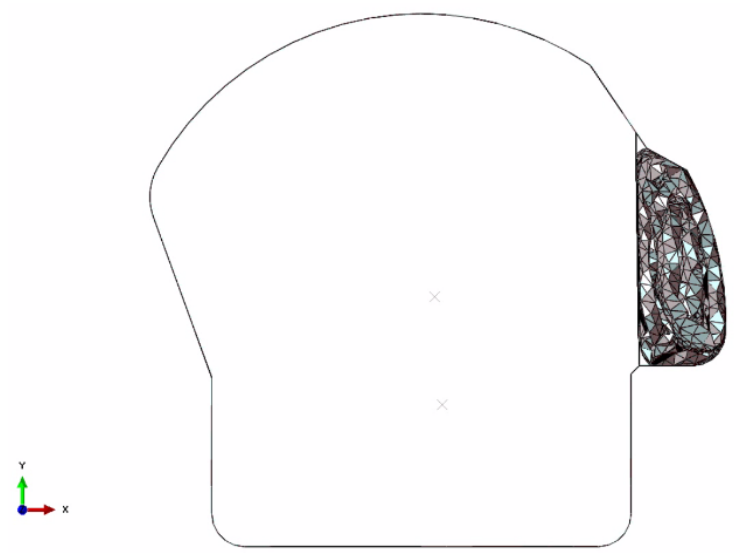

Figure 6.11: Folded plug after implementation of dynamic relaxation. 
From Figure 6.12, it can be seen how the folded inflatable was restored back to $96 \%$ of its initial area $\left(3.6834 \mathrm{~m}^{2}\right)$ of membrane elements within the storage area (Figure 6.1) which limits the relaxation process. In Figure 6.13, there is a spike of the difference percentile between kinetic energy and internal energy when the rigid plane pushes the plug into the storage area. The difference percentile of entire relaxation process is less than $0.5 \%$. Figure 6.14 is the individual plot of kinetic energy of same simulation that shown in Figure 6.13.

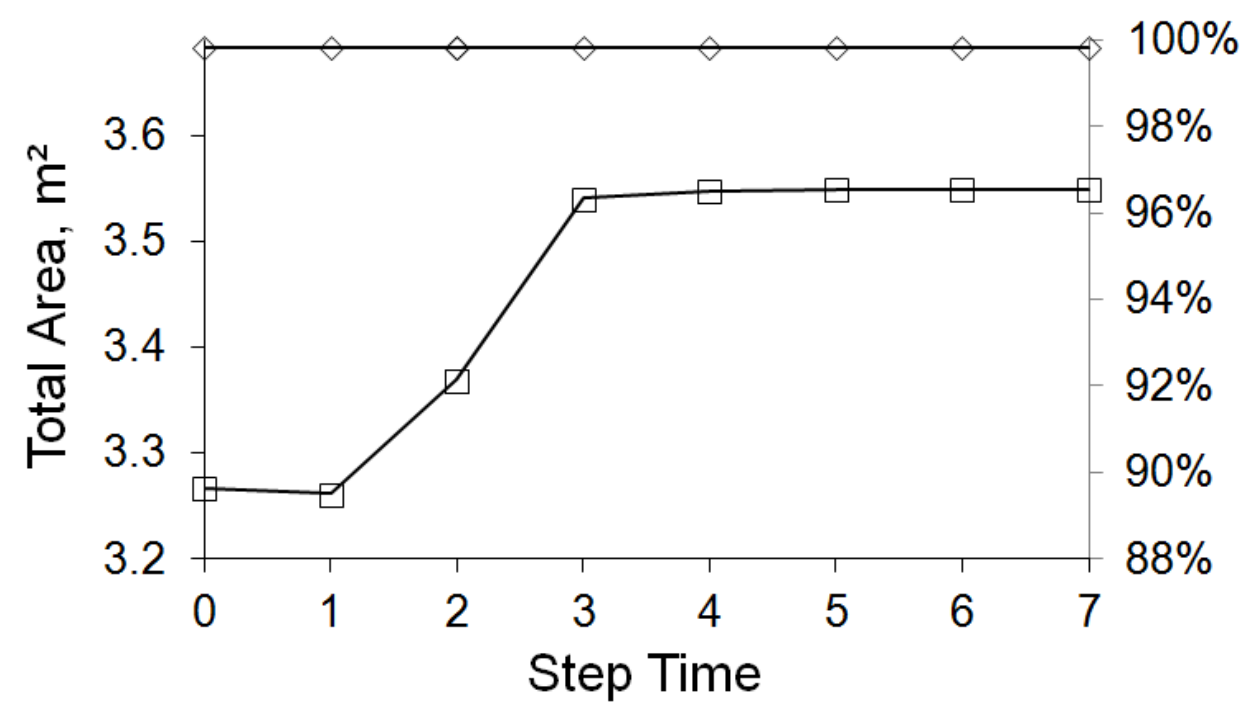

$\rightarrow$ Initial Configuration $\quad \square$ Current Configuration

Figure 6.12: Restitution of Total Area in the Folded plug after relaxation.

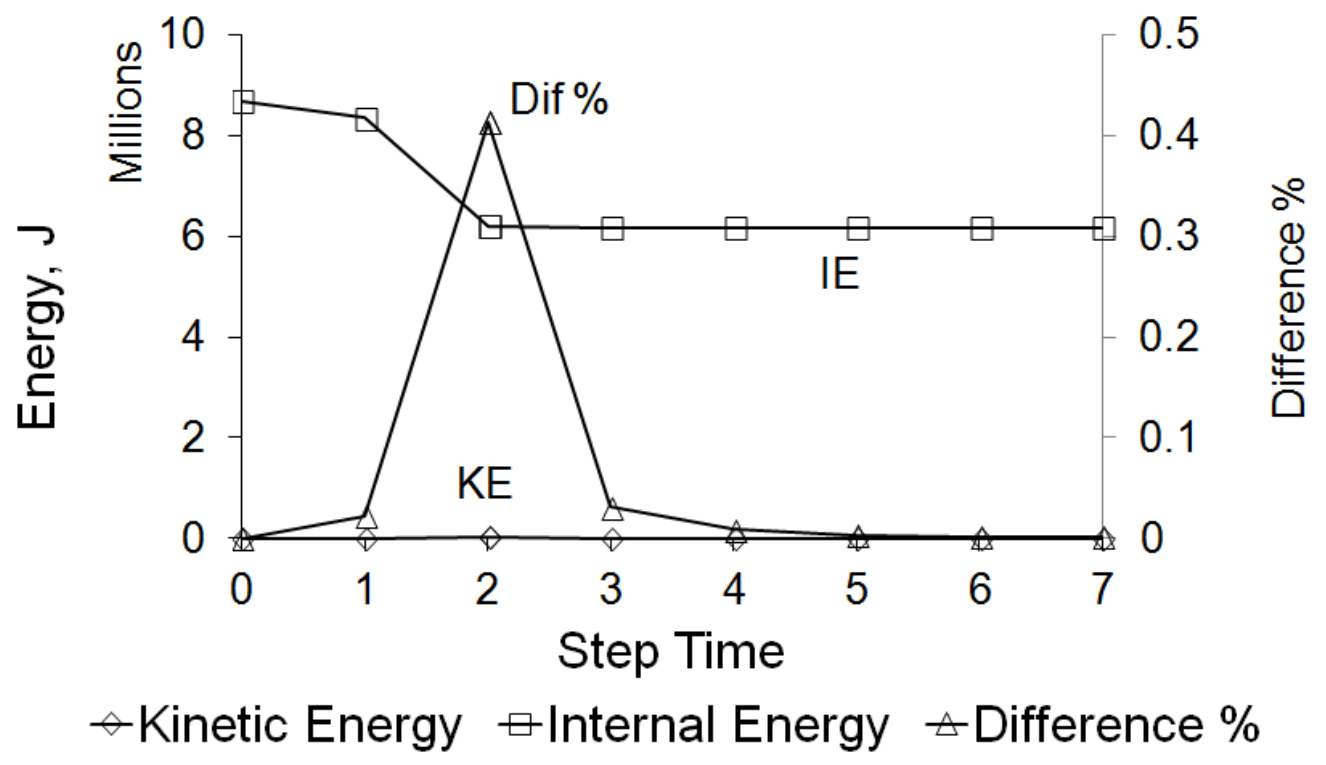

Figure 6.13: Evaluation of Energies during the Implementation of Dynamic Relaxation. 


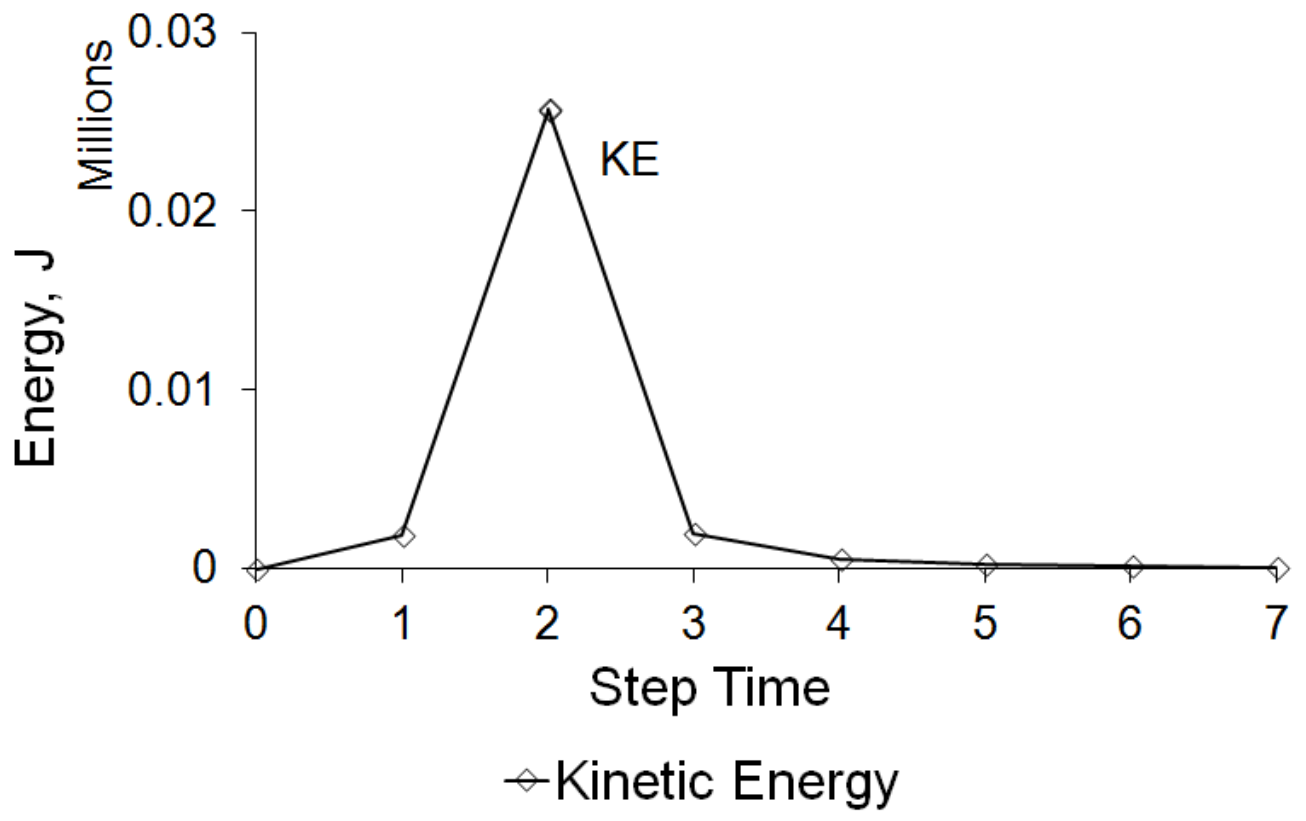

Figure 6.14: Evaluation of Kinetic Energies during the Implementation of Dynamic Relaxation.

The relaxation process will be also added the deployment simulation in order to minimize the kinetic energy, stabilize the simulation process, avoid the distorted shape shown in Figure 6.8, and restore the area of membrane elements in order to reach a value as close as possible to $100 \%$ when the plug is fully inflated inside the tunnel section.

\subsection{Summary}

A combination of translations, rotations and the action of gravity force were applied on the folded plug to place it into the storage area. This approach was successful as the results showed a model with no penetrations or inter-element intersections afterwards. The implementation of the relaxation process demonstrated to be necessary in order to avoid distortion and loss of volume.

The inclusion in the model of a rigid plane representative of the gate allowed to close the storage area and delimited the available volume in which the folded plug had to accommodate after the application of the relaxation process.

Although the folded plug was relaxed once within the enclosed storage area, the total area of membrane elements only restored to $96 \%$ of its initial configuration due to the limitation of enclosed storage area. Therefore, the relaxation process needs to be 
executed again at the stage of deployment, otherwise, the final shape within the tunnel will be distorted, affecting the total area, volume, forces, strains, and stresses in the membrane as shown in Figure 6.8. 


\section{Full-Scale Prototype: Deployment}

\subsection{Introduction}

This chapter presents the simulation of the sequence of deployment of the folded full-scale prototype. It includes the parameters that were necessary to define and adjust in order to obtain results representative of the actual deployment observed experimentally. The sequence begins with the folded inflatable packed in the storage area as described in the previous chapter. When the holding gate opens, the inflation begins, the plug falls out from the storage area, and the folded plug starts to unfold initially by its own weight and by the dynamic force of the fall. The inflation continues the unfolding process while the plug lies on the tunnel floor until it reaches the opposite wall and ceiling of the tunnel section, completing the positioning of the plug inside the tunnel sections.

The development of the deployment simulation required to take several factors into account such as boundary conditions, effect of gravity force, relaxation, friction coefficients, material properties of fabric, inflation methodology, properties of the inflation fluid, and the inclusion of passive restraining mechanisms for sequential deployment of the membrane material in order to achieve acceptable levels of global and local conformity.

The following sections details the purpose of including those factors in the simulation and how they influenced the performance of the deployment.

\subsection{Initial Configuration Before Deployment}

As described in the previous chapter, the folded plug was placed in the storage area as illustrated in Figure 7.1. In this configuration, the folded plug lies on the base of the storage area under the effect of its own weight. A virtual gate holds it up until the deployment sequence is activated. The folded plug is connected to the tunnel section at Line $\mathrm{B}$, described previously, and the nodes of this line are fixed in the $\mathrm{X}, \mathrm{Y}$, and $\mathrm{Z}$ directions as defined during the placement and relaxation stage. This boundary condition represents the ties that fasten and restrain the actual plug to the tunnel section and are assumed to be unbreakable during the deployment simulation. Gravity force and dynamic relaxation are applied on the folded plug from the very beginning of 
the simulation. The gravity force produces the fall of the plug when the virtual gate opens and the relaxation is used to ensure that the surface of folded plug will restore to its original area at the end of the deployment. The tunnel section is assumed to be a rigid body fixed in $X, Y$, and $Z$ direction, so it does not move when it interacts with the plug during deployment.

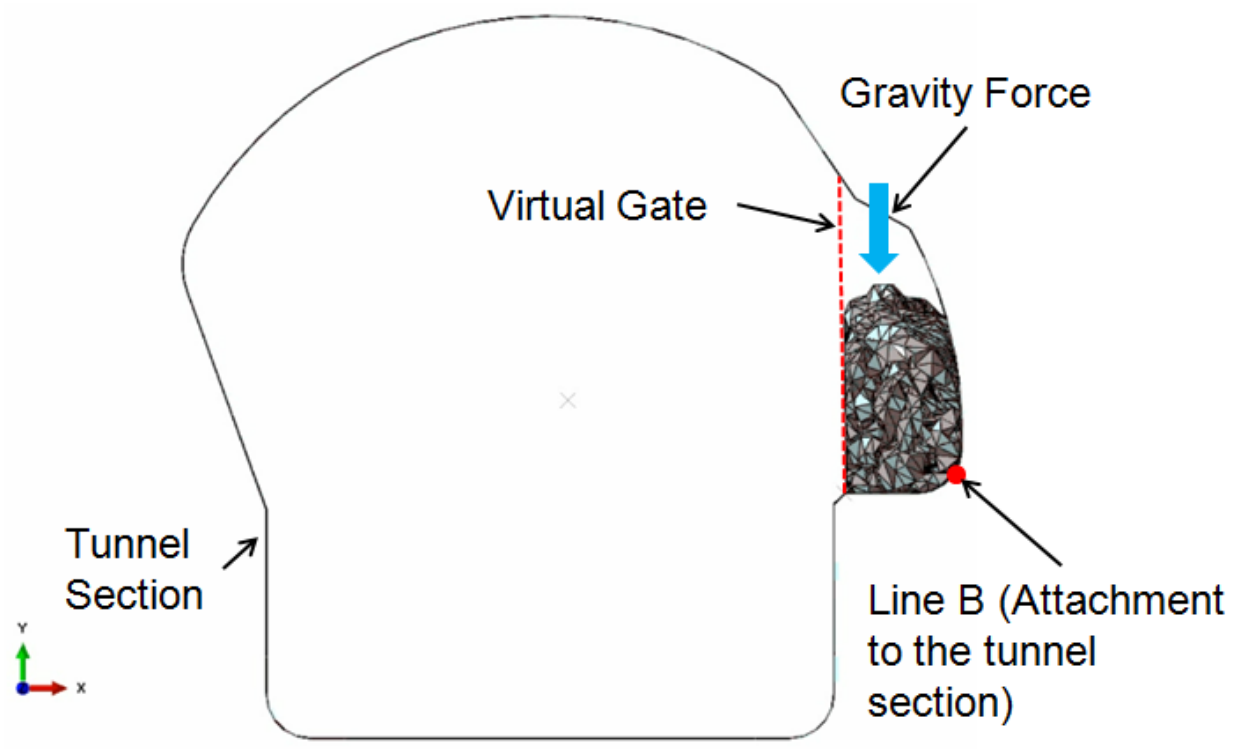

Figure 7.1: Initial position of the folded plug before deployment.

From experimental results, the friction coefficient between plug and tunnel was estimated to be 0.19 [43] and self-friction to the plug itself is 0.21 [54]. These two values defined how the surface of the membrane interacts with itself and with the tunnel surface as the deployment develops in the simulation. The hard contact (explained previously in Chapter 2) was selected to be the contact method between parts.

The material properties of the structural membrane consisting of a single layer fabric representative of the three-layer system (Table 7.1) are taken from Section 3.7.2 and Table 3.3. These equivalent material properties are assigned to the membrane elements of the model. The solid areas representative of metal fittings used to connect the inflation devices have no material properties and they are represented by rigid body elements. 
Table 7.1: Material Properties of Equivalent Membrane

\begin{tabular}{|c|c|c|c|}
\hline Direction & Material Properties & Material \#4 & Stress-Strain Graph \\
\hline \multirow{2}{*}{1} & $\begin{array}{l}\text { Tensile Strength, T11 } \\
\text { (MPa) }\end{array}$ & 258.5534 & \multirow[t]{2}{*}{$\uparrow^{\sigma, \text { Stress }}$} \\
\hline & $\begin{array}{c}\text { Compressive Strength, T22 } \\
\text { (MPa) }\end{array}$ & 25.8553 & \\
\hline & $\begin{array}{l}\text { Tensile Strength, T11 } \\
\text { (MPa) }\end{array}$ & 258.5534 & \multirow{2}{*}{$\begin{array}{l}\left.T_{22}\right|_{\text {, strain }} \\
\varepsilon 11=0.1 \\
\varepsilon 22=0.1\end{array}$} \\
\hline & $\begin{array}{c}\text { Compressive Strength, T22 } \\
(\mathrm{MPa})\end{array}$ & 25.8553 & \\
\hline
\end{tabular}

\subsection{Inflation Methodology}

\subsubsection{Introduction to Inflation Algorithms}

For several years the automotive airbag industry has been using the Finite Element Method to simulate the deployment of airbags under various conditions [115]. The deployment algorithms can be categorized into three different types: the Control Volume Algorithm, the Eulerian-Lagrangian Interaction Algorithm, and the Meshfree Algorithm.

The Control Volume Algorithm is defined by the scalar thermodynamic equations which assume that the gas contained in the chambers is an ideal gas and the formulation of gas flow does not implement a discretization method during the calculations [119]. Wang and Nefske [120] were the pioneers of this concept and developed the algorithm in the late 1980s (well-known later as the Uniform Pressure Method, UPM). Some of the advantages of this method are the easiness of implementation, the relatively low computational cost, and robustness of the simulation. However, this simplified algorithm is not able to simulate local fluid effects because the formulation does not involve the equations of fluid dynamics that describe the movement of fluid [119].

The Eulerian-Lagrangian Interaction Algorithm uses a combination of both the Eulerian algorithm and the Lagrangian algorithm to capture the dynamic effects of the 
simulation in a more precise manner than the UPM does. In general terms, the Lagrangian grid can be deformed and moved with the material, while the Eulerian mesh is fixed. A mesh smoothing is performed because the boundary of the Lagrangian domain is taken to represent the actual interface between the Lagrangian reference grid and the Eulerian frame mesh. Afterwards, the solution of a deformed mesh will take place on the smoothed grid. The grid of the Lagrangian algorithm and the Eulerian algorithm illustrated in Figure 7.2(a) are used to discretize the deforming penetrator (airbag) and the fixed target (gas flow), respectively [115, 121-124]. The two most popular simulation methods that have been developed from the concept of the EulerianLagrangian Interaction Algorithm and used by the airbag simulation industry are the Coupled Eulerian Lagrangian (CEL) method [125-126] and the Arbitrary Lagrangian Eulerian (ALE) method [127-129]. Since the step time of this algorithm cannot be arbitrary large, the computational cost to solve a step is approximately two to five times higher than the Lagrangian step time [130].

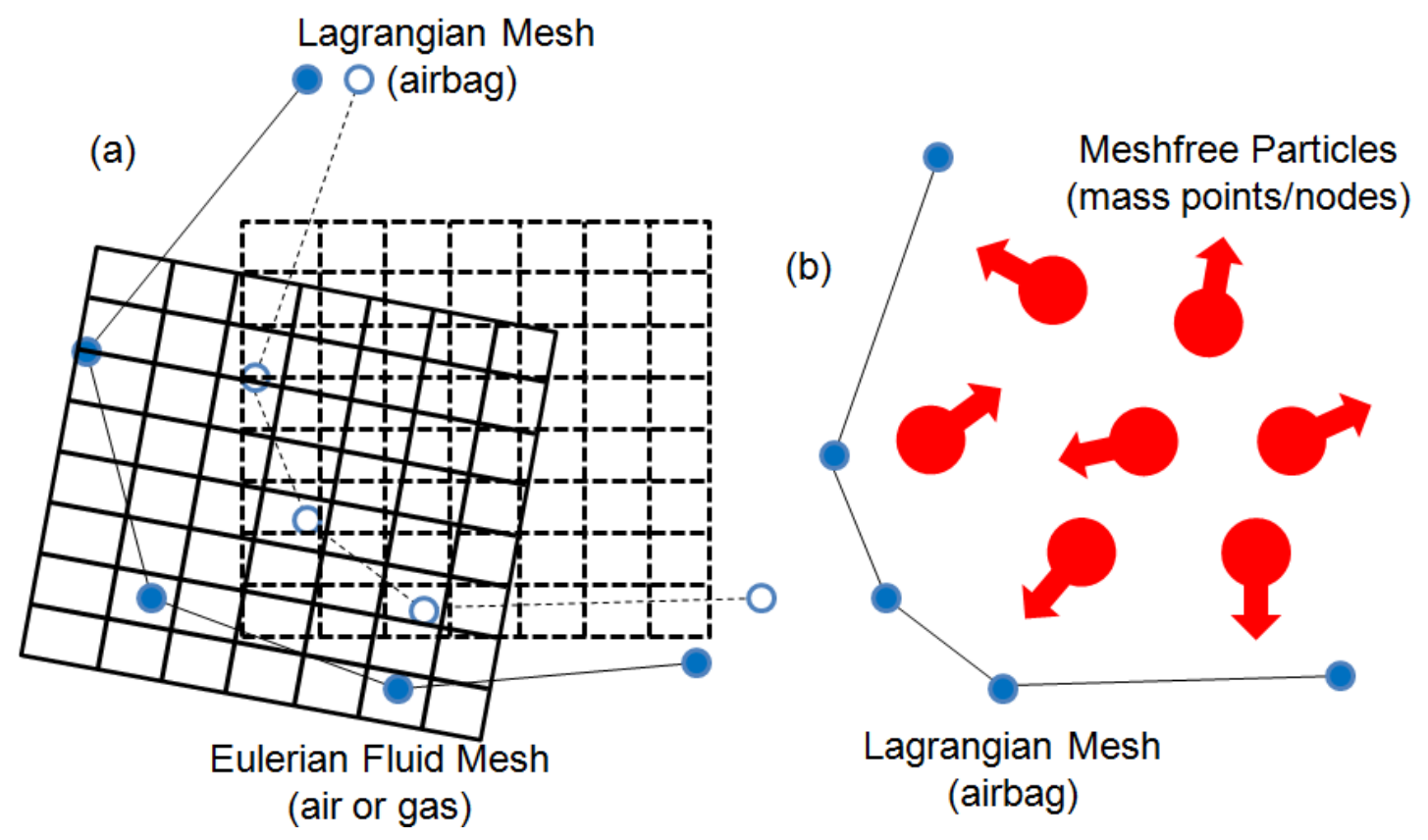

Figure 7.2: (a) Eulerian Lagrangian Interaction Algorithm [119] and (b) Meshfree Algorithm [120]

A meshless and gridless finite element concept called Meshfree algorithm was developed in order to avoid the grid tangling of grid-based algorithms (Lagrangrian and 
Eulerian) in simulations involving large deformations [131-134]. In this algorithm the particles of gas are defined by freely positioned Lagrangian nodes which contain velocity, density, pressure, and temperature. The particles use interpolation points and a weighting function to calculate the physical variables based on the data available from surrounding particles, as schematically illustrated in Figure 7.2(b) [115, 121-124]. Meshfree algorithms such as the Smoothed Particle Hydrodynamics (SPH) [131, 132], or the Finite Pointset Method (FPM) [133], and the Corpuscular Method (C Method) [134] were developed for different purposes and usages. However, the Meshfree algorithms are still considered as an immature approach (mainly because of problems with stability, consistency, and conservation) compared to the grid based Eulerian and Lagrangian algorithms [123].

The complexity of implementation of the Eulerian-Langragian algorithm, as well as the limitations of the Meshfree method, led to the adoption of the Uniform Pressure Method for the simulations carried out in this work. The capability of reproducing the actual deployment behavior with relatively low computational cost and high accuracy is also one of the main factors that led to the use of the UPM as inflation method. Further details on the formulation and implementation are presented in Section 7.3.3.

\subsubsection{Assumptions for the Deployment Simulation}

Most of the pre-simulation conditions of the full-scale prototype are similar to the ones that are typically applied to airbag's simulations [53-68]. However, additional assumptions have been made to define and simplify some of the conditions of the simulation based on the behavior observed during actual deployments of full-scale inflatables used for protection of tunnels [58]. These assumptions include:

- Gravity force is applied on the entire plug along the Z-axis from the beginning to the end of the simulations (Figure 7.1).

- There is no other gas than air at ambient temperature of $80^{\circ} \mathrm{F}$ and ambient pressure of $1.0133 \mathrm{MPa}$ used to inflate the plug.

- The volumetric values of air flow and inflation time are based on experimental results; the values used for the simulations are $0.8495 \mathrm{~m}^{3} / \mathrm{sec}\left(1800 \mathrm{ft}^{3} / \mathrm{min}\right)$ and 200 seconds (3.33 minutes) [58], respectively. 
- The fabric is assumed to be impermeable. Therefore, no leakage event will be considered in this simulation. Literally, there will be zero outgoing air from the plug and the efficiency of the air inflow is $100 \%$ during the inflation process.

- Inflow air behaves as an ideal gas without heat transfer into the fluid cavity (adiabatic process).

- The air is assumed to be transferred into and within plug through an orifice which has a discharge coefficient of 1.0 .

- The incoming air enters into the plug through the chamber with the inflation fittings. Section 7.3.7 explains how the fluid flows within the chambers.

- The relaxation process applies to the model during entire deployment simulation.

- The chamber walls are used to calculate the volume of fluid within the plug only and do not have physical contact to any solid part in the model.

- The nodes connecting the plug to the tunnel section are fully constrained through boundary condition settings on the tunnel wall.

- As mentioned previously, the friction coefficient between plug and tunnel is 0.19 [59] and fabric to fabric is 0.21 [60].

\subsubsection{Uniform Pressure Method}

The Uniform Pressure Method (UPM) was the approach selected to perform the inflation simulation of the full-scale prototype. The implementation of this method is relatively simple and involves a relatively lower computational cost when compared to more sophisticated methods described in previous sections [119]. Wang and Nefske [120] developed the UPM based on the ideal gas law and assuming an adiabatic process to determine the pressure of pneumatic fluids within a domain (chamber) as a function of density. The absolute pressure is determined by:

$\tilde{p}=\rho R\left(\theta-\theta^{Z}\right)$

where the absolute pressure, $\tilde{p}$, can be also expressed as

$\tilde{p}=p+p_{A}$ 
where $p_{A}$ is ambient pressure, $R$ is gas constant, $\theta$ is current temperature, $\theta^{Z}$ is absolute zero on the temperature scale being used. The gas constant, $R$, can be defined by the universal gas constant, $\tilde{R}$, and the molecular weight, $M W$ as:

$R=\frac{\tilde{R}}{M W}$

The change of inflating fluid mass in the fluid cavity, $\dot{m}$ consists of three components,

$\dot{m}=\dot{m}_{\text {in }}-\dot{m}_{\text {out }}$

where $\dot{m}_{i n}$ is mass rate conveyed into the fluid cavity domain of the internal plug and $\dot{m}_{\text {out }}$ is the mass rate that exits from the fluid cavity domain of the internal plug [114]. Assuming an impermeable fabric and the absence of leakages on the surface of the fabric during the inflation, the change of inflating fluid mass in the fluid cavity can also be simplified as the mass flow rate conveyed into the fluid cavity domain directly. That is $\dot{m}=\dot{m}_{\text {in }}$

Abaqus/Explicit computes the fluid temperature from the predefined temperature at the cavity reference node and from conservation of energy in an adiabatic process. Therefore, no heat transfer activity will be expected during the transferring of inflating fluid mass. The first law of thermodynamics is used to calculate the energy of fluid cavity [43] and the energy equation of fluid cavity at time $t$ can be written as,

$\frac{d(m E)}{d t}=\dot{m}_{\text {in }} H_{\text {in }}-\dot{m}_{\text {out }} H_{\text {out }}-\dot{W}-\dot{Q}$

where $m, E, H_{\text {in }}, H_{\text {out }}, \dot{W}$, and $\dot{Q}$ denote the mass of fluid cavity, specific energy, specific enthalpy into fluid cavity, specify enthalpy out from fluid cavity, work done by the fluid cavity expansion, heat energy flows out through the surface of fluid cavity, respectively [43]. Equation 7.6 can be simplified to

$\frac{d(m E)}{d t}=\dot{m}_{i n} H_{i n}-p \dot{V}-\dot{Q}$

where the work done by the fluid cavity expansion, $\dot{W}=p \dot{V}$, can be replaced by pressure, $p$, and volumetric flow rate of fluid cavity, $\dot{V}$. The specific energy, $E$, can be expressed as

$E=E_{i}+\int_{\theta_{i}}^{\theta} c_{v}(T) d T$ 
where $E_{i}, \theta_{i}$, and $c_{v}$ indicate the initial specific energy, initial temperature, constant volume heat capacity, respectively. Also, the equation of specific enthalpy of fluid cavity, $H$, can be stated as

$H=H_{i}+\int_{\theta_{i}}^{\theta} c_{p}(T) d T$

where $H_{i}$ is the initial specific enthalpy at the initial temperature and $c_{p}$ is the constant pressure heat capacity. Both constant volume and pressure heat capacities are depending only upon temperature for an ideal gas [43].

To model an adiabatic process for the ideal gas, the constant pressure heat capacity, $c_{p}$, need to be defined. The polynomial form of the Shomate equation is implemented to calculate the molar constant pressure heat capacity, $\tilde{c}_{p}$ [135]. According to the National Institute of Standards and Technology [135], the polynomial form of the molar constant pressure heat capacity can be expressed as:

$\tilde{c}_{p}=\tilde{a}+\tilde{b} T+\tilde{c} T^{2}+\tilde{d} T^{3}+\frac{\tilde{e}}{T^{2}}$

or $\quad \tilde{c}_{p}=\tilde{a}+\tilde{b}\left(\theta-\theta^{Z}\right)+\tilde{c}\left(\theta-\theta^{Z}\right)^{2}+\tilde{d}\left(\theta-\theta^{Z}\right)^{3}+\frac{\tilde{e}}{\left(\theta-\theta^{Z}\right)^{2}}[43]$

where $T=\left(\theta-\theta^{Z}\right)$ has units of temperature (Kelvin)/1000, the values of coefficients $\tilde{a}$, $\tilde{b}, \tilde{c}, \tilde{d}$, and $\tilde{e}$ are constants. The physical properties of air used for the models are listed in Table 7.2. Then, the constant pressure heat capacity can be computed by $c_{p}=\frac{\tilde{c}_{p}}{M W}$

and the constant volume heat capacity can be obtained by $c_{v}=c_{p}-R$

One of the main simplifications of the UPM is that it assumes that the internal pressure of the plug is constant at any time step. This simplification reduces the computational cost. However, the local gas motion cannot be represented by the constant pressure algorithm [119]. 
Table 7.2: Physical Properties of Air [136-139].

\begin{tabular}{|c|c|c|c|}
\hline Symbols & Description & Value & Unit \\
\hline \multirow{2}{*}{$M W$} & \multirow{2}{*}{ Molecular Weight } & 28.97 & $\frac{\mathrm{kg}}{\mathrm{kmol}}$ \\
\hline & & 28.97 & $\frac{l b m}{l b m o l}$ \\
\hline \multirow{2}{*}{$\tilde{a}$} & \multirow{10}{*}{ Coefficients of Shomate Equation } & 28.11 & $\frac{\mathrm{kJ}}{\mathrm{kmol} . \mathrm{K}}$ \\
\hline & & 62686.2035 & $\frac{i n . l b f}{l b m o l . R}$ \\
\hline \multirow{2}{*}{$\tilde{b}$} & & $0.1967 \mathrm{E}-02$ & $\frac{k J}{k m o l . K}$ \\
\hline & & 2.4363 & $\frac{i n . l b f}{l b m o l . R}$ \\
\hline \multirow{2}{*}{$\tilde{c}$} & & $0.4802 \mathrm{E}-05$ & $\frac{k J}{k m o l . K}$ \\
\hline & & 3.3057E-03 & $\frac{i n . l b f}{l b m o l . R}$ \\
\hline \multirow{2}{*}{$\tilde{d}$} & & $-1.966 \mathrm{E}-09$ & $\frac{k J}{k m o l . K}$ \\
\hline & & $-7.52 \mathrm{E}-07$ & $\frac{i n . l b f}{l b m o l . R}$ \\
\hline \multirow{2}{*}{$\tilde{e}$} & & 0.0 & $\frac{k J}{k m o l . K}$ \\
\hline & & 0.0 & $\frac{i n . l b f}{l b m o l . R}$ \\
\hline \multirow{2}{*}{$\tilde{R}$} & \multirow{2}{*}{ Universal Gas Constant } & 8.3143 & $\frac{k J}{k m o l . K}$ \\
\hline & & 18540 & $\frac{i n . l b f}{l b m o l . R}$ \\
\hline \multirow{2}{*}{$\theta$} & \multirow{2}{*}{ Current Temperature } & 273-1800 & ${ }^{\circ} K$ \\
\hline & & $491-3240$ & ${ }^{\circ} \mathrm{R}$ \\
\hline \multirow[t]{2}{*}{$\theta^{Z}$} & \multirow{2}{*}{ Absolute Temperature } & 0.0 & ${ }^{\circ} \mathrm{K}$ \\
\hline & & 0.0 & ${ }^{\circ} \mathrm{R}$ \\
\hline \multirow{3}{*}{$\theta_{i}$} & \multirow{3}{*}{ Ambient Temperature } & 299.8167 & ${ }^{\circ} \mathrm{K}$ \\
\hline & & 539.67 & ${ }^{\circ} R$ \\
\hline & & $\begin{array}{c}80 \\
266667\end{array}$ & ${ }^{\circ} \mathrm{F}$ \\
\hline \multirow[b]{2}{*}{$p_{A}$} & \multirow{2}{*}{ Ambient Pressure } & $\begin{array}{l}26.666 \% \\
1.01325\end{array}$ & $M P a$ \\
\hline & & 14.7 & $p s i$ \\
\hline \multirow{3}{*}{$\rho_{\text {air }}$} & \multirow{3}{*}{ Density of Air } & 1.229 & $\mathrm{~kg} / \mathrm{m}^{3}$ \\
\hline & & 4.4256E-05 & $\mathrm{lbm} / \mathrm{in}^{3}$ \\
\hline & & $1.1464 \mathrm{E}-7$ & $\frac{l b f \cdot \sec ^{2}}{i n^{4}}$ \\
\hline
\end{tabular}




\subsubsection{Multi-Chamber and Fluid Exchange Setup}

The advanced automotive airbags are developed using multiple chambers in order to control the inflation shape and speed [143-144, 147]. The multiple chambers of an airbag can be inflated with a pre-determined order at a pre-determined speed in order to protect a the passenger [143]. In order to have a full continuity of gas flow from one chamber to the other without any restriction, Lienard and Lefevre [145] proposed to create four chambers linked to each other and to use material-less shell elements as chamber walls to create partitions between chambers in a rolled inflatable tube model (Figure 7.3). Slade et al. [146] divided an airbag simulation model with 6 chambers by using impermeable radial diaphragms as chamber walls to allow more differential inflation strategies to handle various landing situations on Mars (Figure 7.4).

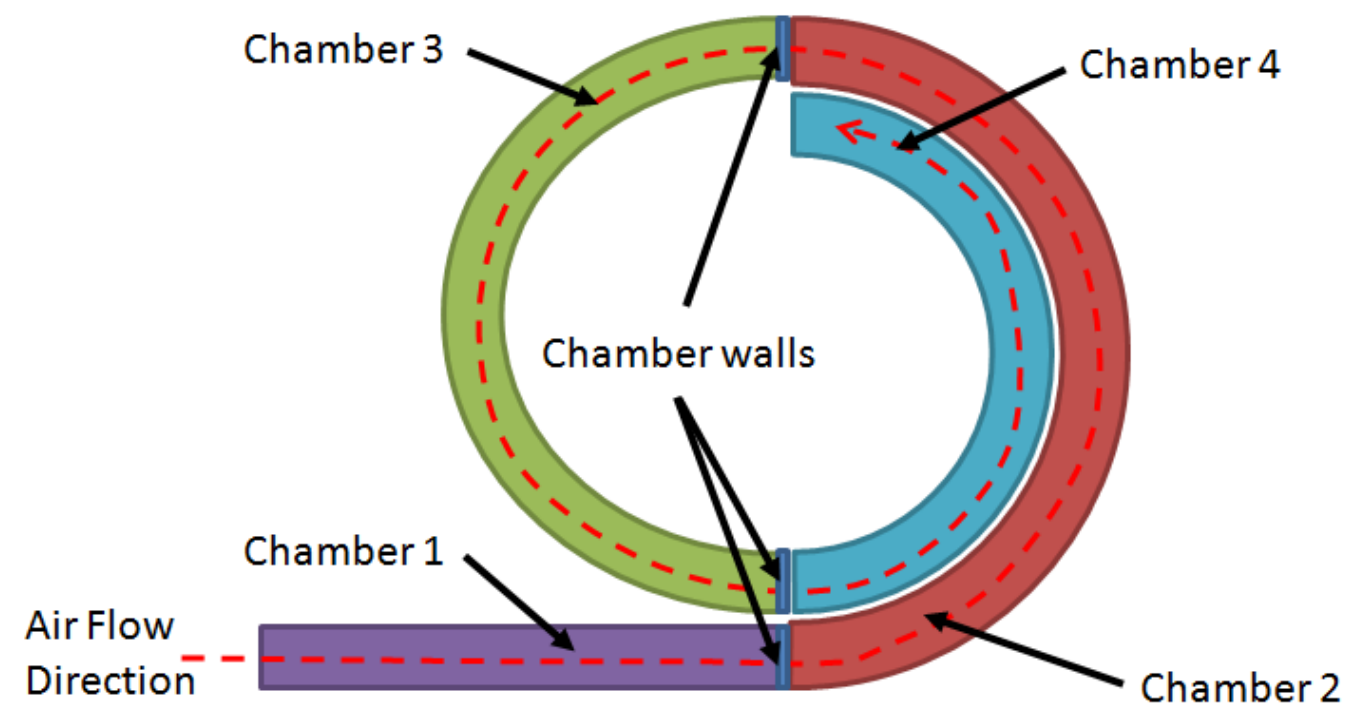

Figure 7.3: Definition of multi-chamber in an inflatable tube [145].
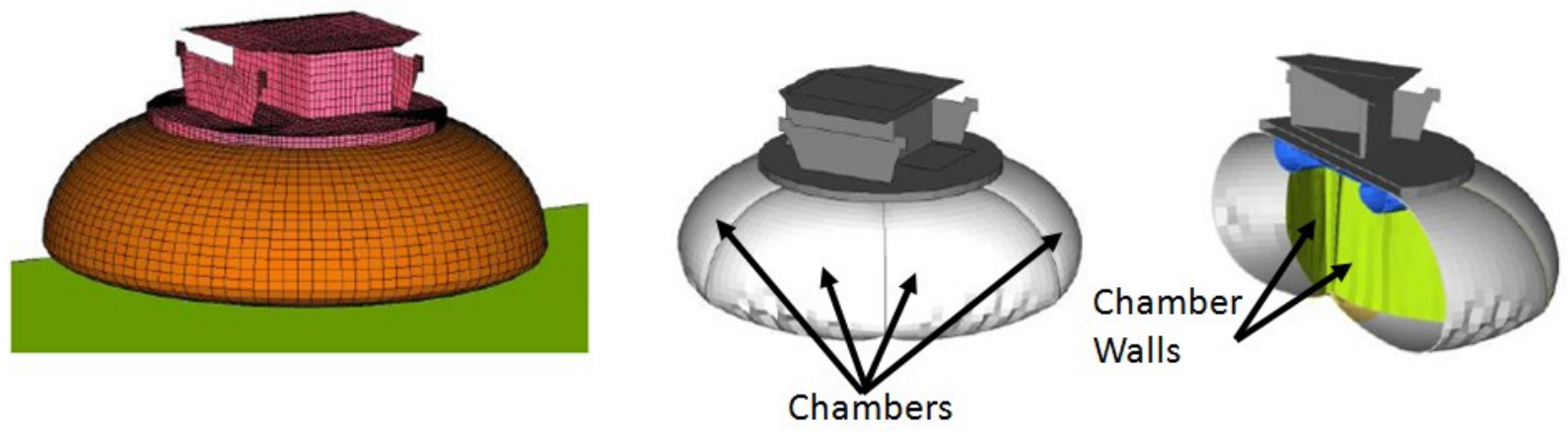

Figure 7.4: Definition of multi-chamber in an inflatable modeled by Slade et al. [146]. 
For the work developed in this dissertation, a multi-chamber approach was used to define the direction of fluid flow inside the plug since the gas motion inside the plug cannot be simulated by the Uniform Pressure Method [140-141]. The flow direction inside the plug needed to be defined in order to improve the deployment behavior thus making it more similar to reality. The multi-chamber approach with fluid flowing between chambers through holes or fabric leakage has been implemented in other fluid-filled systems such as automotive airbags [43]. The multi-chamber approach consists of dividing the single physical domain of the plug into multiple chambers using fictitious walls to obtain a gradient of pressure across the physical domain [43]. The fictitious fluid flow or leakage mechanisms can be defined through fictitious holes in the internal walls to replicate actual deployment behavior [43]. Abaqus uses the surface facet geometry (fictitious walls in this case) and the cavity reference node to calculate the volume of the fluid cavity that interacts with the internal surface facet geometry of a chamber. The surface facet geometry of the chamber is constructed by surface elements [43].

Surface elements are placed on facets, edges, or ends of elements. The elements can be defined as deformable or rigid according to the characteristics of the surface. The surface can be partially deformable and partially rigid when deformable surface elements have been assigned underlying a surface of a rigid body [43]. Abaqus can compute the contact stress acting on the surface by associating the surface area with each node when surface elements are used in a mechanical contact analysis [43].

Besides defining the fluid cavity for each enclosed chamber, the appropriate fluid exchange definition needs to be included to connect the fluid cavities together to perform a multi-chamber approach [43]. Fluid exchange definitions are implemented to model the transfer between two fluid cavities or between a single fluid cavity and its environment either as a prescribed function or based on the pressure difference arising from analysis conditions [43]. The flow between a fluid cavity and its environment in the model represents the air flow coming from the air hose connected to the plug through the fitting ports installed in the actual prototype. On the other hand, the flow within the plug can be determined as the transfer between two fluid cavities.

The flow between a single fluid cavity and its environment can be defined as the relationship between the single reference node with the primary cavity [43]. The flow 
can be designed to transfer into or out of the primary cavity when it is defined as a prescribed function [43]. The material properties of the flow going in are assumed to be the instantaneous material properties in the cavity itself [43]. The heat energy flow can be either into or out of the primary cavity but the mass flow can be only out of the primary cavity, based on instantaneous analysis conditions [43]. Abaqus/Explicit defines the flow behavior based on mass flow rate or heat energy flow [43]. Abaqus determines the mass flow rate by using the fluid cavity pressure and specified constant ambient pressure to calculate the pressure difference for the case of mass flow [43]. Furthermore, Abaqus defines the heat energy flow rate by using the fluid cavity temperature and the specified constant ambient temperature to calculate the temperature difference for the case of heat energy flow [43].

The flow between two fluid cavities can be defined by associating the reference nodes with the primary and secondary fluid cavities [43]. The fluid flows from high (upstream) pressure cavity to low pressure cavity and the heat energy flows from high (upstream) temperature to low (downstream) temperature, based on instantaneous analysis conditions [43].

The mass flow is assumed to be one-dimensional, quasi-steady, and isentropic flow (adiabatic and reversible) through an orifice [43, 142]. The equation of mass flow rate associated to an orifice can be expressed as follows,

$|\dot{m}|=C A \frac{\tilde{p}_{e}}{\sqrt{R\left(\theta-\theta^{Z}\right)}} \sqrt{\frac{2 \gamma}{\gamma-1}\left(q^{\frac{2}{\gamma}}-q^{\frac{\gamma+1}{\gamma}}\right)}$

where $C$ is the dimensionless discharge coefficient, $A$ is the orifice area, $\theta$ is the temperature in the upstream fluid cavity, $\theta^{Z}$ is the absolute temperature, $\gamma$ is the ratio between the constant pressure heat capacity and the constant volume heat capacity $\left(\gamma=\frac{c_{p}}{c_{v}}\right)$, and $\tilde{p}_{e}$ is the absolute pressure in the upstream fluid cavity [43]. The pressure ratio, $q$, can be determined as,

$q=\frac{\tilde{p}}{\tilde{p}_{e}}$

where $\tilde{p}$ is the absolute pressure in the orifice. The critical pressure, $p_{c}$, associated with the absolute pressure in the upstream fluid cavity at which choked flow occurs for 
adiabatic conditions (the mass flow rate increases when the upstream pressure increases or the upstream temperature decreases). The critical pressure can be expressed as

$p_{c}=\tilde{p}_{e}\left(\frac{2}{\gamma+1}\right)^{\frac{\gamma}{\gamma-1}}$

The absolute pressure in the orifice, $\tilde{p}$, is given by

$\tilde{p}=p_{a} ; \quad$ if $p_{a} \geq p_{c}$

$\tilde{p}=p_{c} ; \quad$ if $p_{a}<p_{c}$

where $p_{a}$ is the ambient pressure of a single fluid cavity for flowing out or the downstream cavity pressure for transferring between two fluid cavities. The value of the dimensionless discharge coefficient can be calculated as the function of the absolute upstream pressure, temperature, or any user-defined field variables [43].

Abaqus/Explicit is capable of modeling the flow characteristics and the position of inflators used for the deployment of inflatable structures [43]. The inflator can be used to inflate a fluid cavity that represents the actual inflator used as airbag supplemental restraints systems [43]. The inflator also can be used to inflate a fluid cavity with a mixture different ideal gas [43]. The simulation output of mass flow rate can be identified using inflator feature and the activation timing can be controlled through inflator during the inflation analysis [43]. The mass flow rate and temperature are the inflator properties which associate with the function of inflation time directly [43].

\subsection{Volume Flow Rate Verification}

In order to verify the physical properties of air listed in Table 7.2, the full-scale prototype was inflated in constrained conditions and the resulting volume was compared to the volume obtained for an unconstrained inflation. The volume for an unconstrained inflation is approximately $150.898 \mathrm{~m}^{3}$ (39863 gallon, $\left.5329 \mathrm{ft}^{3}\right)$ and the time to reach such volume during the experiments was nearly 180 seconds [58]. The inflation process in this verification model under constrained conditions lasted for 180 seconds and the volume reached for that time was compared to the volume corresponding to an unconstrained inflation. It is expected that the constraining effect of the tunnel section 
will prevent reaching $100 \%$ of the volume corresponding to an unconstrained condition, but that effect should appear only in the final stage of the inflation.

The FE model was set to have volume flow rate of $0.8495 \mathrm{~m}^{3} / \mathrm{sec}\left(1800 \mathrm{ft}^{3} / \mathrm{min}\right)$ of air flowing into the plug during the inflation process as it was in the physical tests [58]. The simulation requires a mass flow rate in order to complete the calculation of energy. Then, the mass flow rate can be calculated from volume flow rate as,

Volume flow rate of air, $\dot{V}=0.8495 \mathrm{~m}^{3} / \mathrm{sec}$

$$
\begin{aligned}
& =\dot{m} / \rho_{\text {air }} \\
& =\dot{m}_{\text {in }} / \rho_{\text {air }} \\
\text { Mass flow rate of air, } \dot{m}_{\text {in }} & =\dot{V} * \rho_{\text {air }} \\
& =0.8495 \mathrm{~m}^{3} / \mathrm{sec} * 1.229 \mathrm{~kg} / \mathrm{m}^{3} \\
& =1.0440 \mathrm{~kg} / \mathrm{sec}=2.2942 \mathrm{lbm} / \mathrm{sec}
\end{aligned}
$$

In order to compensate the dynamic response of mass scaling (introduced for computational cost reduction purposes discussed in Chapter 5) and resistance of gas stream due to narrow bridges between chambers [144], a scale factor needs to be implemented into the mass flow rate [145]. The scale factor $\left(S_{f}\right)$ of inflation flow rate can be defined as the ratio between the theoretical output of mass flow ( $\left.\dot{m}_{\text {theoretical }}\right)$ and the numerical output of mass flow ( $\left.\dot{m}_{\text {numerical }}\right)$ as follows:

Scale factor, $S_{f}=\frac{\dot{m}_{\text {theoretical }}}{\dot{m}_{\text {numerical }}}$

Figure 7.5 shows the evaluation of the volumetric flow rate for three different models: unconstrained plug, constrained plug without scale factor, and constrained plug with scale factor. The unconstrained plug had a mass flow rate increment of 0.8495 $\mathrm{m}^{3} / \mathrm{sec}$ and achieved the original volume (approximately $150 \mathrm{~m}^{3}$ ) in the expected time (approximately 180 seconds), while the constrained plug without scale factor reached only nearly $45 \mathrm{~m}^{3}$, which corresponds to error of $66.5 \%$.

Using the average difference volumetric flow rate from the model of constrained plug without scale factor, a scale factor, $S_{f}$, of 3.4578 is implemented into the mass flow rate of air and the difference of volumetric plot line has reduced to $2.8 \%$ before the plug starts to be constrained in the tunnel, around the $100^{\text {th }}$ second. 
The efficiency of volumetric flow rate is assumed to be $100 \%$ and no fabric leakage for all these three models. Then, for the deployment simulation, the volume flow rate of $0.8495 \mathrm{~m}^{3} / \mathrm{sec}$ with scale factor, $S_{f}$, of 3.4578 will be used to define the flow rate of air during inflation.

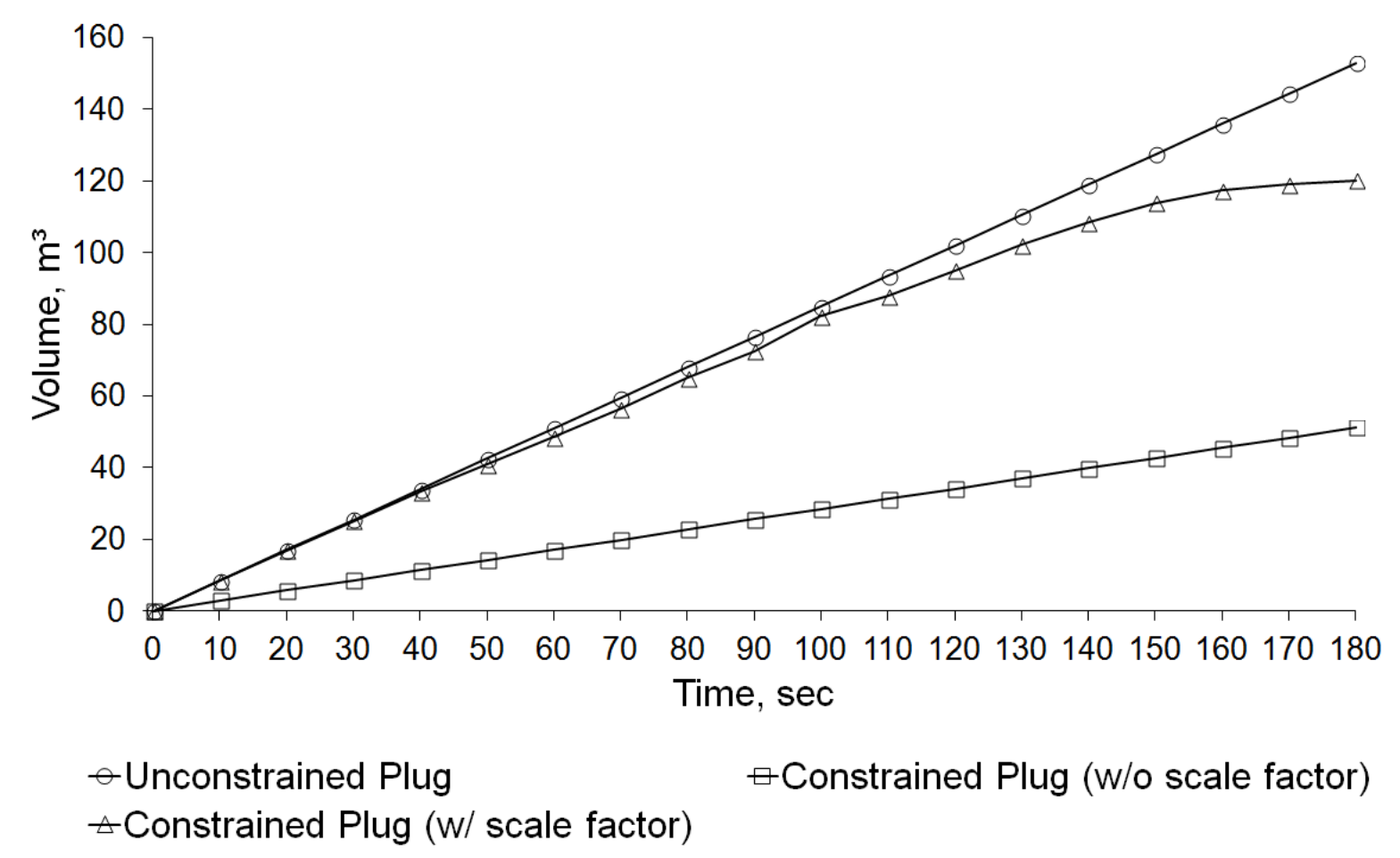

Figure 7.5: Flow rate verification.

\subsection{Passive Restraining Mechanism}

As previously mentioned in Chapter 5, a passive restraining mechanism consisting of multiple ties were installed in the surface of the inflatable during the folding process. The purpose of this ties was to produce a sequential release of membrane material in order to improve the level of local conformity in the upper region of the tunnel. Fifteen ties were used to connect the edges of the folded plug defined by line $C$ and line $D$ (see Figure 5.1). Each tie has a nominal tensile strength of $778.4387 \mathrm{~N}(175 \mathrm{lbf})$, an elongation of $20.32 \mathrm{~mm}$ ( $0.8 \mathrm{in}$ ), which provides an elastic constant of $38.309 \mathrm{~N} / \mathrm{mm}$ (218.75 lbf/in). Both tensile strength and elastic constant need to be scaled by 386 in order to achieve force equilibrium at the stage of deployment since the mass scaling factor of 386 (Chapter 5) has implemented into the model initially. 
The ties were represented by connector elements previously described in Chapter 2. The ties are assumed to have translational degrees of freedom during the deployment simulation. In order to reduce the complexity of the model, a simplified approach of connection was selected to represent and replicate the deformation behavior of the ties. The connector element CONN3D2 and the simplified translational basic connection component denominated AXIAL were selected.

The elastic connector behavior uses the spring stiffness of to define the elasticity of the connection,

$F_{i}=D_{i i} u_{i}$ with $i=1,2,3$

where $F_{i}$ is the force in the $i$ component of relative motion, $D_{i i}$ is the spring stiffness matrix components, and $u_{i}$ is the displacement or rotation in the $i$ direction [42-43]. The numerical elastic stiffness (force per relative displacement with scale factor of 386 ) used in the models is $14787.274 \mathrm{~N} / \mathrm{mm}$ (84437.5 lbf/in).

The failure of a connector releases the relative motion based on failure criterion in terms of relative position, force, or moment in the direction of the component. The failure force with scaled factor of 386 used to define the breaking point of a connector element in the model is $300477.3382 \mathrm{~N}(67550 \mathrm{lbf})$.

\subsection{Modeling of Deployment}

This section describes the model implemented for the deployment of the fullscale prototype. The evaluation of the effect of the passive restraining mechanism on the deployment behavior is analyzed. The level of local conformity is evaluated by computing the contact area between plug and tunnel wall. The effect on the deployment behavior of directing the air flow within plug is compared with the available experimental data reported in [58]. All these parameters were adjusted in order to reproduce experimental results. Figure 7.6 shows a sequence of an actual deployment of a fullscale prototype tested in the WVU testing facility [58]. 


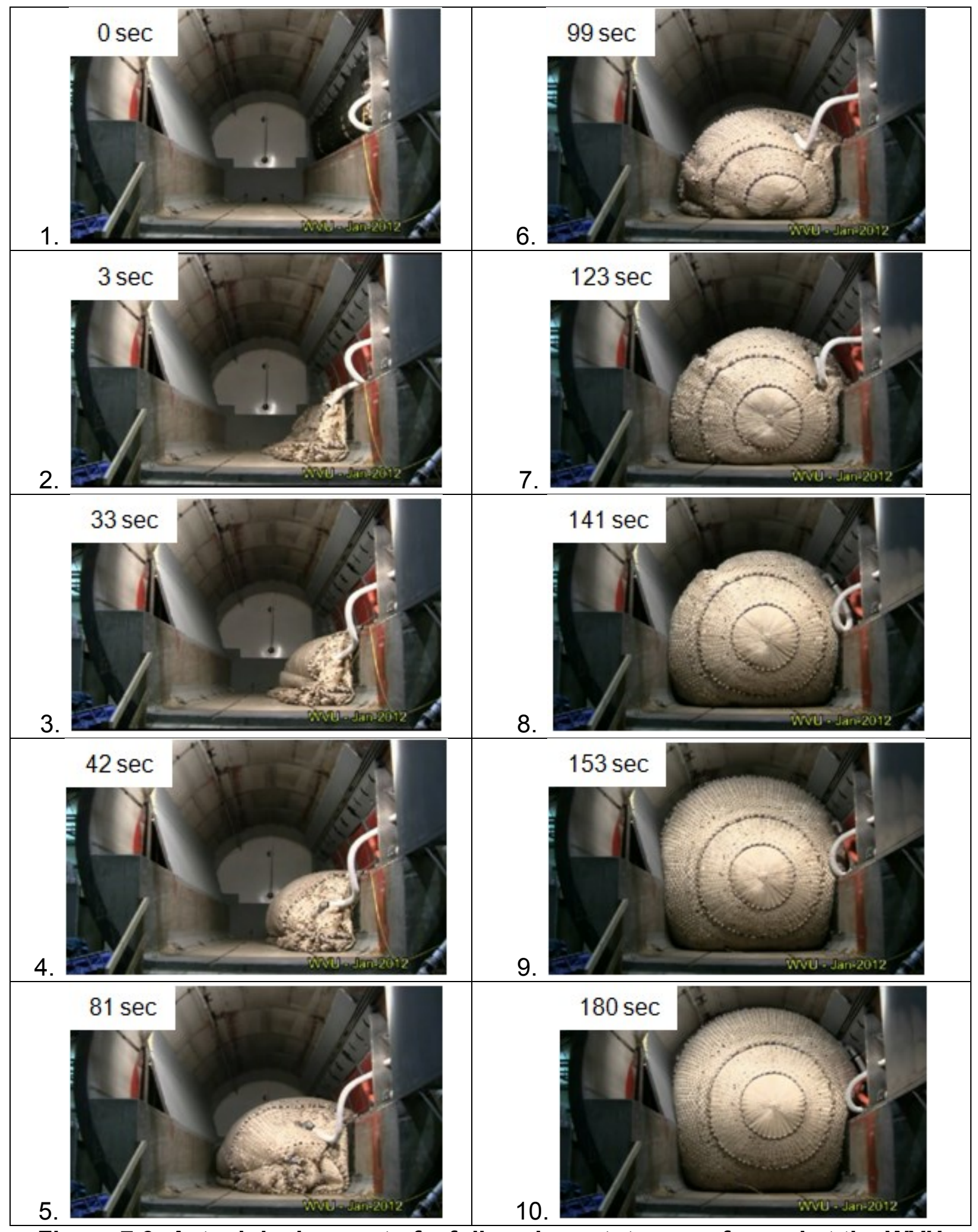

Figure 7.6: Actual deployment of a full-scale prototype performed at the WVU testing facility [58] 
The folded plug in storage position, described in Chapter 6, was used as starting point of the deployment simulation. Several models were generated considering different conditions in order to evaluate their influence in the performance of the deployment. The test plan showing various deployment conditions is summarized in Table 7.3.

Table 7.3: Test Plan of Deployment Simulations.

\begin{tabular}{|c|c|c|}
\hline Deployment \# & Connector Elements & Air Flow Directed \\
\hline 1 & No & No \\
\hline 2 & Yes, without mass scaling & No \\
\hline 3 & Yes, with mass scaling & No \\
\hline 4 & Same as Deployment 3 & Yes \\
\hline
\end{tabular}

All the deployment models summarized in Table 7.3 have the same properties of air indicated in Table 7.2, the same verified volume flow rate of air described in Section 7.4 , and the inflation time lasts for 200 seconds which is similar to the inflation time obtained experimentally (180 seconds) [58]. The extra simulation time is to allow stabilization of the inflatable once it reaches the final position within the tunnel section. Figure 7.7 shows the individual chambers separated by the chamber walls that were created inside the plug. In this model the inflation port is located in Chamber 2.

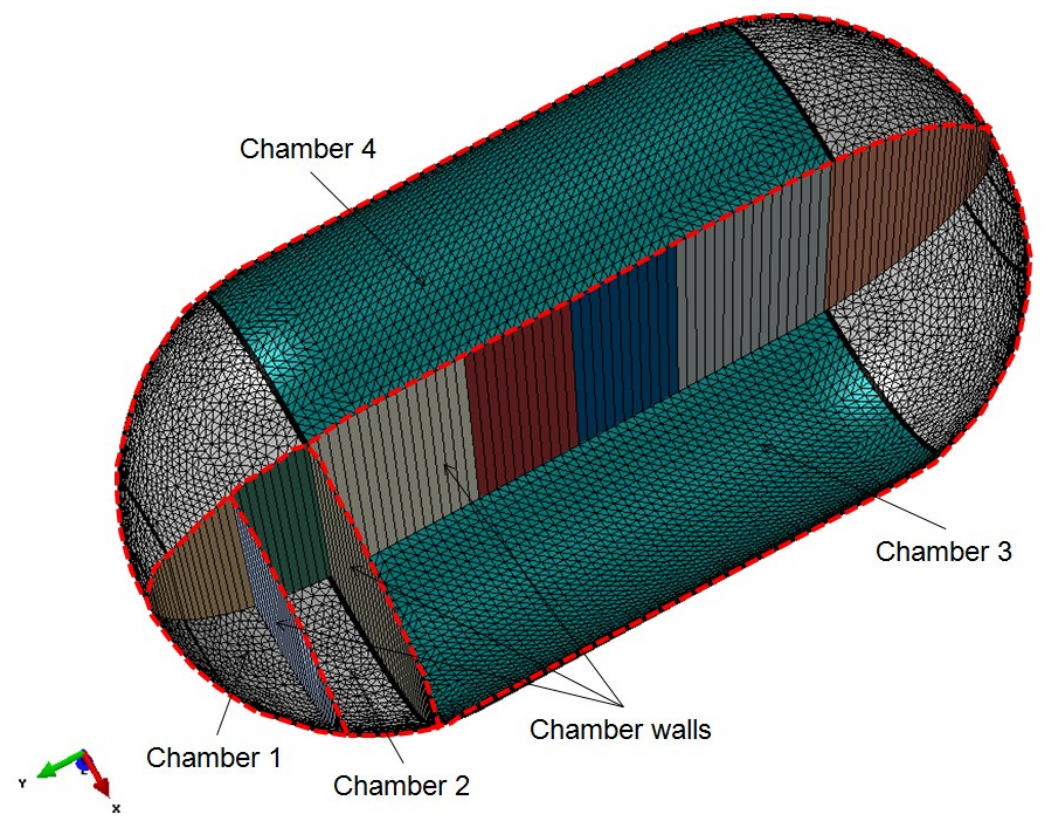

Figure 7.7: Position of chamber walls. 
The folded plug in Deployment \#1 does not include connector elements representative of ties to control the release of material as described in Chapter 5. Moreover, the air flow is not directed within the plug. When the air flow is not directed, the air fills up the plug evenly, spontaneously, and immediately once it enters into Chamber 2, as illustrated in Figure 7.8. The uniform pressure is calculated and applied on the entire internal surface of the plug when the air flow is not directed within the plug.

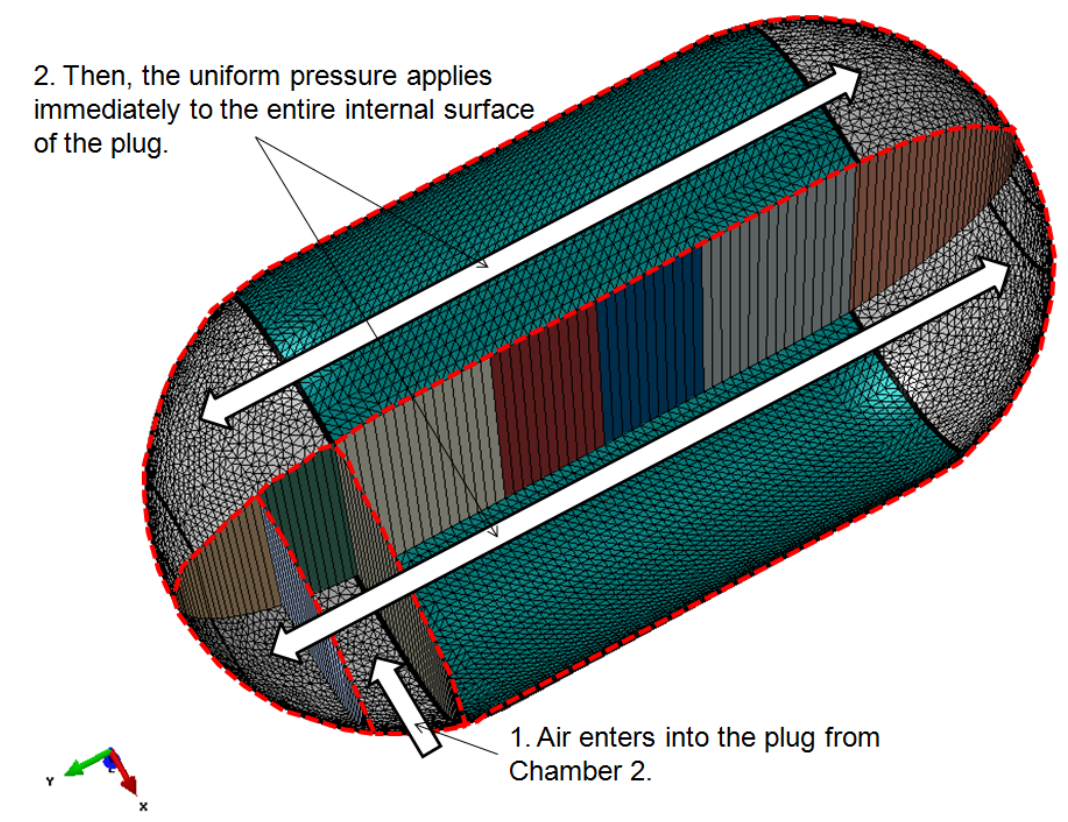

Figure 7.8: Undirected air flow schema (Deployments \#1, \#2, and \#3).

Deployment \#2 included fifteen connector elements placed as described in Chapter 4 and the elastic stiffness and failure strength are $38.309 \mathrm{~N} / \mathrm{mm}(218.75 \mathrm{lbf} / \mathrm{in})$ and $778.4387 \mathrm{~N}$ (175 lbf), respectively. As mentioned in session 7.5, a scale factor of 386 was applied to the elastic stiffness and failure strength of connector elements in Deployment \#3 in order to compensate the dynamic response associated to the mass scaling factor. The connector elements in Deployment \#3 included a scaled elastic stiffness and a scaled failure force of $14787.274 \mathrm{~N} / \mathrm{mm}$ (84437.5 lbf/in) and $300477.3382 \mathrm{~N}$ (67550 lbf), respectively.

Deployment \#4 is basically taken from Deployment \#3, but with a specific fluid exchange design to direct the air flow within the plug. From Figure 7.9, the air fills up the first half (from Chamber 1 to Chamber 3 ) immediately after entering into the plug. After this initial step, the second half (Chamber 4 ) of the plug starts inflating at the $20^{\text {th }}$ 
second. The time of fluid exchange in the simulation was adjusted based on observations of the actual deployment behavior. The inflation time range of the $15^{\text {th }} \sim 20^{\text {th }}$ second is typical timing that worked well to replicate the deployment nearly to the actual inflation. As the actual deployment shown in Figure 7.4 (1) and (2), half of the plug is pinched under the weight of fabric up to 20 seconds or more and the bottom of plug started filling up at the $33^{\text {rd }}$ second (Figure 7.4 (3)). So, it is unreasonably to expect pressure to reach the pinched part. However, a FE model without chambers or extreme narrow bridges between chambers would put pressure everywhere within the internal surface uniformly.

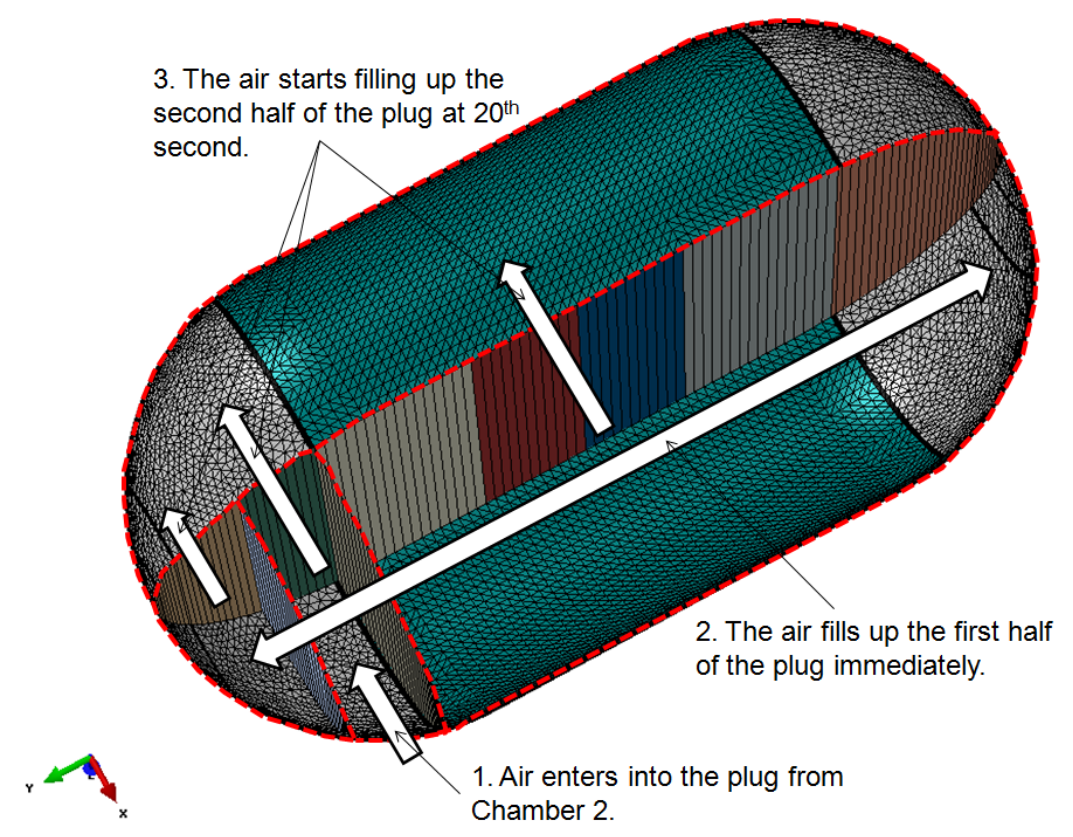

Figure 7.9: Directed air flow schema (Deployment \#4).

\subsection{Results}

As reference, Figure 7.10 shows details of local conformity and position of wrinkles obtained experimentally at WVU [58]. Local conformity at different critical places such as corners and transitions is illustrated in Figure 7.10(a). This image shows a successful case of local conformity because the membrane of the plug is not bridging, and therefore is not leaving open gaps, in all the critical spots. Figure 7.10 (b) displays the position of a longitudinal wrinkle on the wall after implementation of the passive restraining mechanism which led to better local conformity in the upper portion of the 
tunnel section. These results are used as a points of comparison with the results of the FE models.

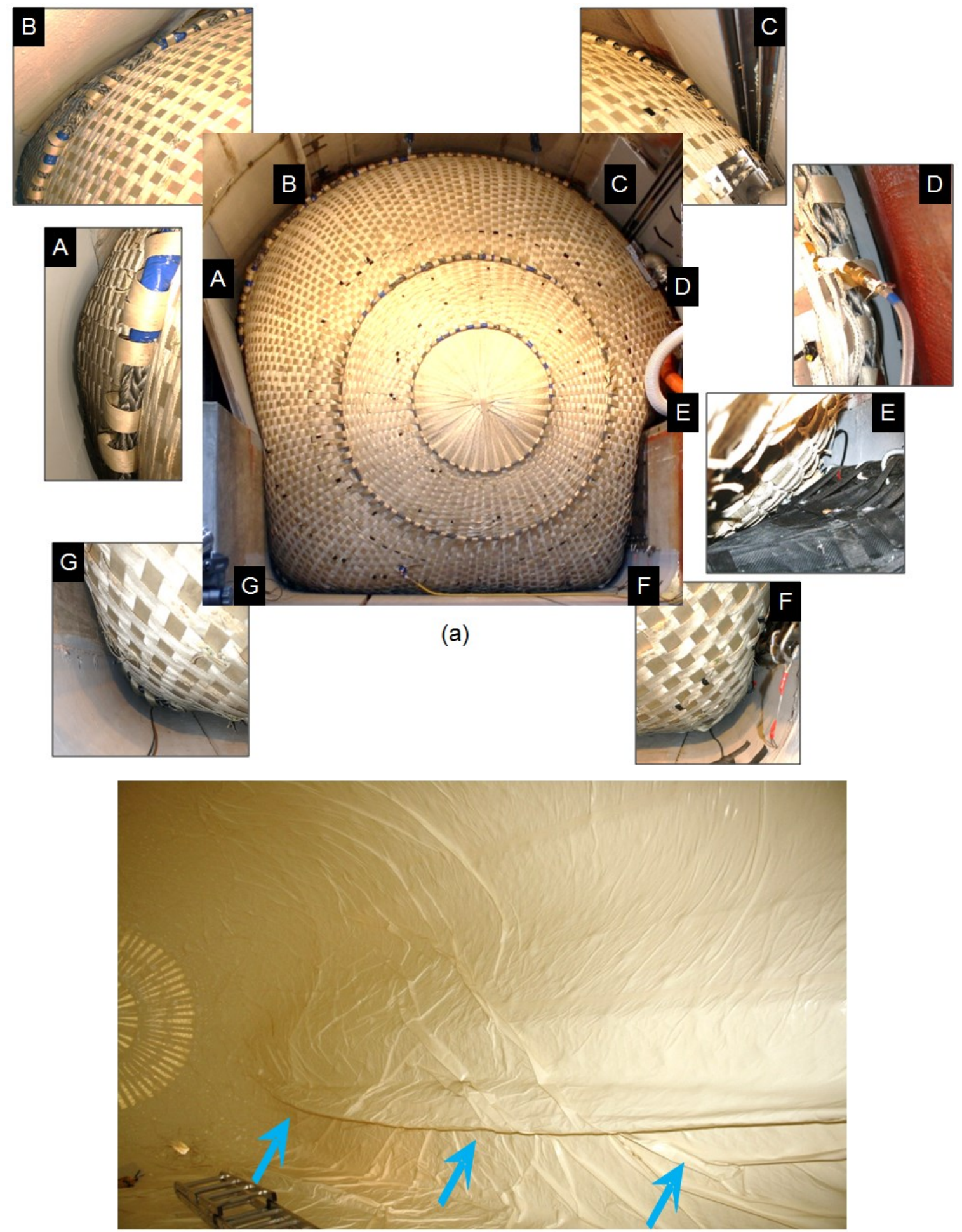

(b)

Figure 7.10: Actual deployment. (a) Front view with details of local conformity, and (b) Wrinkle view from the interior of the plug. 
Figures 7.11 to 7.14 display the detailed of local conformity of each deployment case summarized in Table 7.3 at the end of the inflation (200 ${ }^{\text {th }}$ second). Longitudinal cuts of the deployed plug show the location of wrinkles for the different cases.

Simulation results show that the local conformity of Deployment \#1 leaves at least three visible bridging spots at critical corners and also accumulates wrinkles at one corner of the floor (Figure 7.11). Deployment \#2 predicted slightly less wrinkles and shows two visible bridging spots compared to Deployment \#1 (Figure 7.12). In Deployment \#2, the implementation of the connector elements into the model seems to be helping in driving more material to the upper part of the tunnel and therefore helping to reduce the amplitude of the bridging in corners.

Deployments \#3 (Figure 7.13) and \#4 (Figure 7.14) for which the strength of the connector was scaled by a factor of 386 , do not show any visible bridging spot at the critical corners. However, Deployments \#3 and \#4 do have small longitudinal wrinkles that occurred on the tunnel wall instead of corner of floor when compared to Deployments \#1 and \#2. These results are indicative that the connectors are now working and driving up the membrane material of plug from the floor to the vertical walls during the inflation. The excess of membrane material forms wrinkles on the tunnel wall instead of corner of the floor when the connector elements break at the $158^{\text {th }}$ second, which is similar to what was observed experimentally [58].

The fluid exchange definition does not seem to affect the result of local conformity by comparing Deployment \#3 and Deployment \#4 (Figure 7.14), however, note that the wrinkle pattern obtained in Deployment \#4 resulted similar to the experimental result illustrated in Figure 7.10.

The occurrence of wrinkles is in part related to how the membrane material is distributed and driven during the deployment, but it is also function of the amount of oversizing left in the perimeter of the cylindrical portion of the plug during the manufacturing process to account for unforeseen elements in the tunnel that can add to the perimeter to seal, or for the non-uniformity of the deployment process that can lead to irregular distribution of membrane material. 

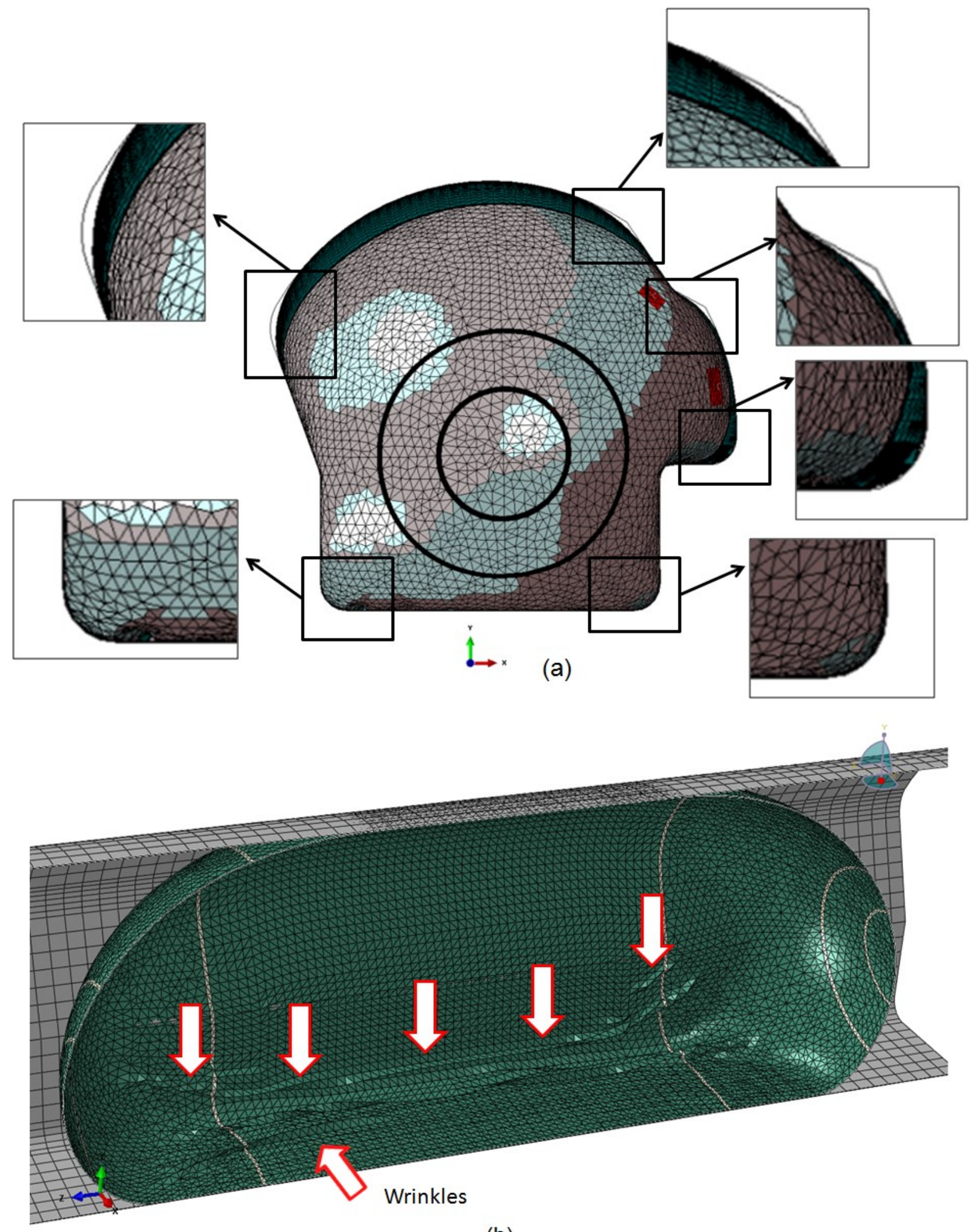

(b)

Figure 7.11: Deployment \#1. (a) Front view and (b) Wrinkle view. (Deformation Scale Factor $=1$ ) 

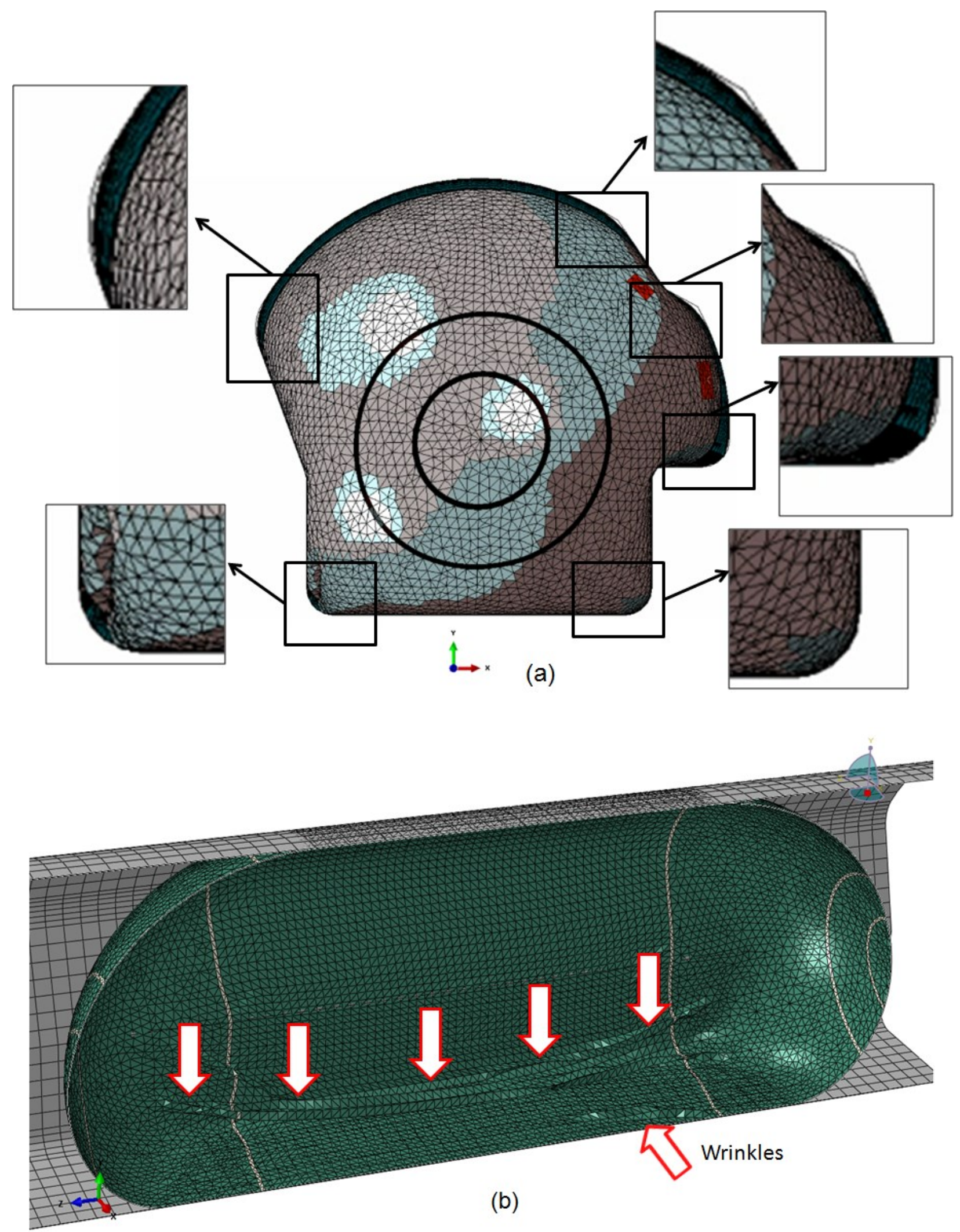

Figure 7.12: Deployment \#2. (a) Front view and (b) Wrinkle view. (Deformation Scale Factor $=1$ ) 


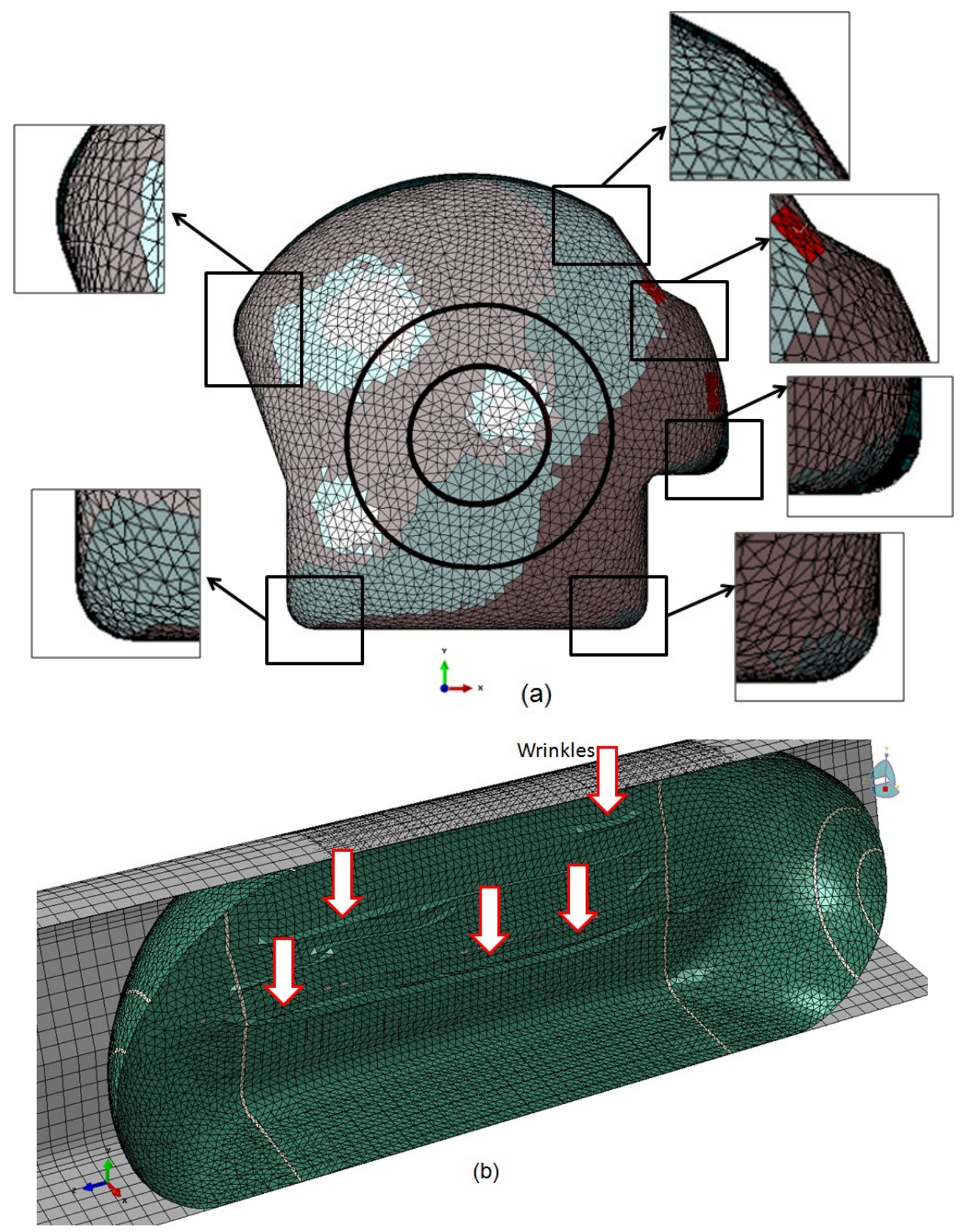

Figure 7.13: Deployment \#3. (a) Front view and (b) Wrinkle view. (Deformation Scale Factor $=1$ ) 


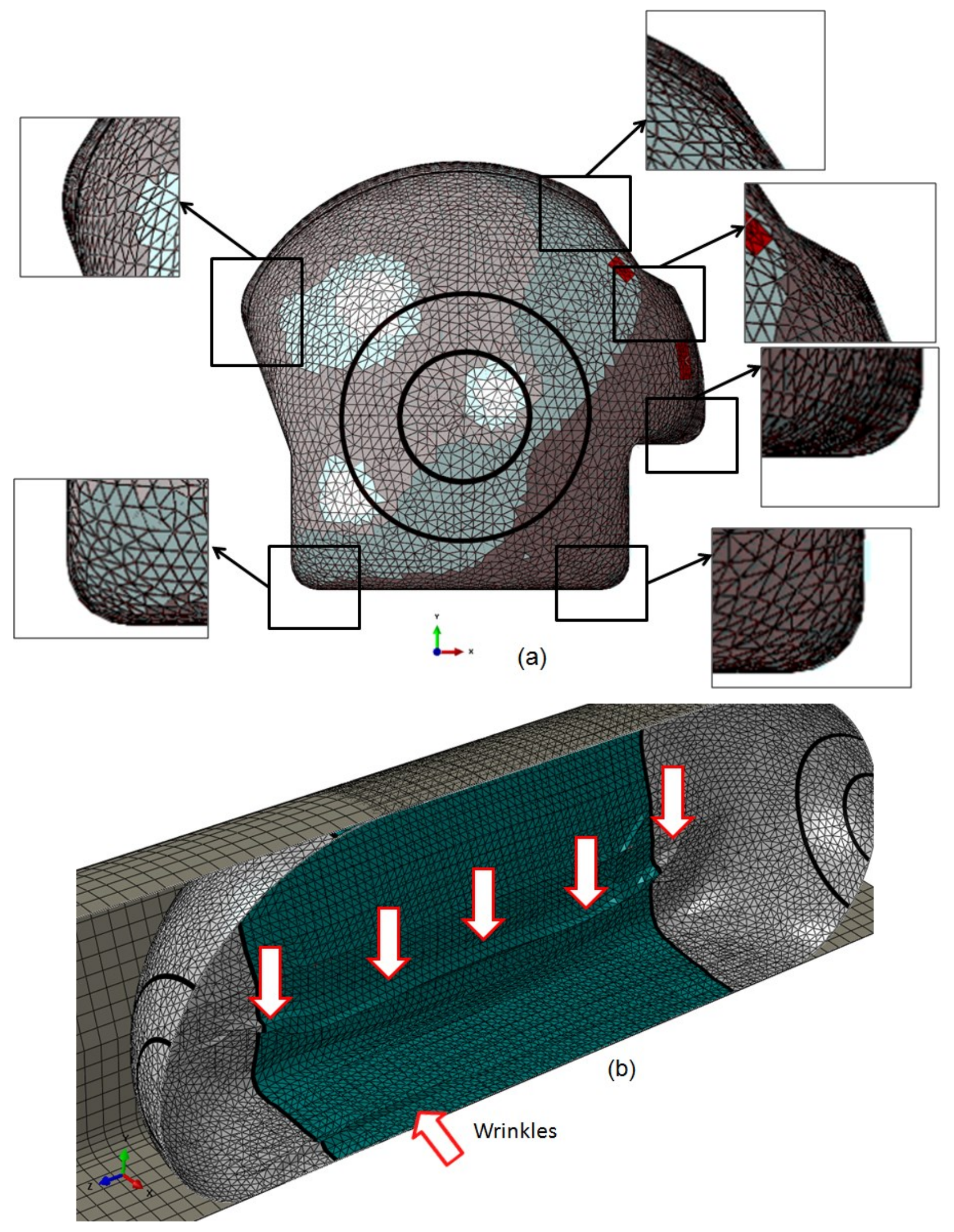

Figure 7.14: Deployment \#4. (a) Front view and (b) Wrinkle view. (Deformation Scale Factor $=1)^{2}$ 
In order to quantify the level of global and local conformity of the plug to the tunnel section, the actual contact area was computed for each deployment. Abaqus has the capability of computing the contact area between two objects, which in this case corresponding to the inflated plug and the tunnel wall. Table 7.4 summarizes the contact area for each deployment case. The cylindrical portion of the plug is designed to have theoretical contact area of $71.9792 \mathrm{~m}^{2}\left(774.7776 \mathrm{ft}^{2}\right.$, see Chapter 4). This area should provide sufficient slippage resistance to handle an external force that will try to push the plug along the tunnel section that the plug itself is intending to seal [15].

Table 7.4: Contact area after completion of deployment.

\begin{tabular}{|c|c|c|c|c|}
\hline Deployment & 1 & 2 & 3 & 4 \\
\hline $\begin{array}{c}\text { Unconfined Inflation } \\
\text { Cylindrical Contact Area }\left(\mathrm{m}^{2}\right)\end{array}$ & 71.9792 & 71.9792 & 71.9792 & 71.9792 \\
\hline $\begin{array}{c}\text { Confined Inflation } \\
\text { Simulation Contact Area }\left(\mathrm{m}^{2}\right)\end{array}$ & 81.5147 & 91.4914 & 95.2701 & 96.2740 \\
\hline Contact Enhancement \% & $+14 \%$ & $+27 \%$ & $+32 \%$ & $+34 \%$ \\
\hline Visible Bridging Spots & 3 & 2 & 0 & 0 \\
\hline
\end{tabular}

Deployment \#1 shows an increase of the contact area of $14 \%$ respect to the original design contact area, even though the model did not include any connector elements to drive up membrane material which created at least three bridging spots in the upper portion of the tunnel, as observed in Figure 7.11. The extra contact area is generated by the confining effect of the tunnel section on part of the hemispherical endcaps which contributed to the increasing of the total contact area. The connector elements implemented in Deployment \#2 failed earlier than expected. Still, the contact area increased to $27 \%$ and reduced the visible bridging spots to two at the critical corners. From the results of contact area, clearly the addition of connector elements has improved the local conformity by nearly duplicating the percentage of increase of contact area with respect to Deployment \#1.

Deployment \#3 included connectors with scaled elastic stiffness and failure force. These connector elements broke at the $158^{\text {th }}$ second and left no bridging spots. Deployment \#3 has a contact enhancement of $32 \%$ with respect to the theoretical value. 
Deployment \#4 predicted a contact enhancement slightly higher $2 \%$ than Deployment \#3. In Deployment \#4, directing the air flow within the plug by opening the chamber 4 at $20^{\text {th }}$ second (Figure 7.9), did not produce obvious differences in the deployment of the plug when folded as described in Chapter 5. However, Deployments \#3 and \#4 are the closest to the experimental results. Unfortunately, the experimental contact area is not easily measurable and it is not available for comparison with the simulation results. Despite this is limitation, the simulation results show the advantage of using FE modeling to predict or estimate physical quantities that probably cannot be obtained directly from experimental results.

The kinetic and internal energy as well as the total area of membrane elements were recorded for Deployment \#4 during the inflation analysis. Figure 7.15 shows the plots of kinetic $\left(E_{k}\right)$ and internal $\left(E_{i}\right)$ energies as well as the ratio $\left(\frac{E_{k}}{E_{i}}\right)$ between these two energies. The ratio of energies $\left(\frac{E_{k}}{E_{i}}\right)$ shows spikes when the air pushes the folded plug away from one corner to the other $(6.38 \%$ at $t \cong 10 \mathrm{sec})$ as well as the moment when ties are broken $(3.43 \%$ at $\mathrm{t} \cong 170 \mathrm{sec})$. However, the percentile of spikes is lower than $10 \%$ and the average ratio percentile of entire inflation analysis is $0.18 \%$. Figure 7.16 shows the total area of membrane elements has successfully restored to $99.6 \%$ of the initial configuration at the end of the simulation. Once again, the inclusion of mass scaling and dynamic relaxation in the deployment simulations are considered successful.

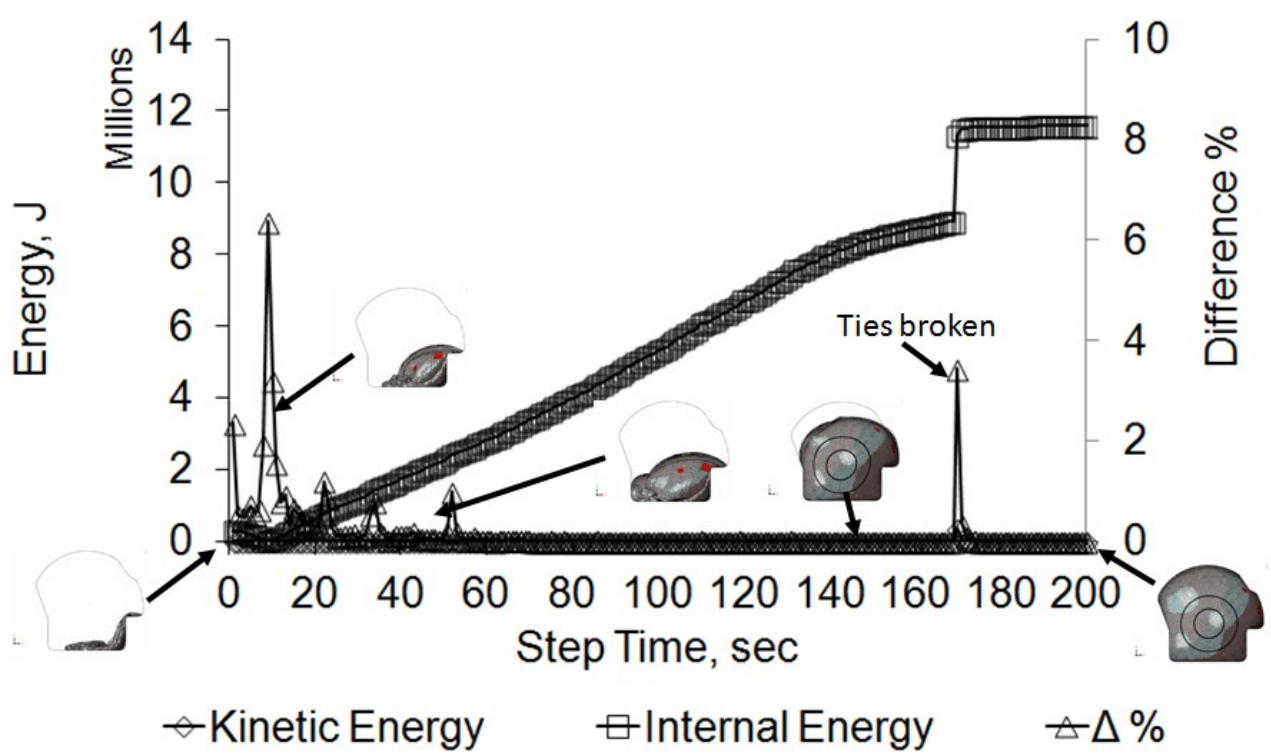

Figure 7.15: Energy Trace of Deployment \#4 


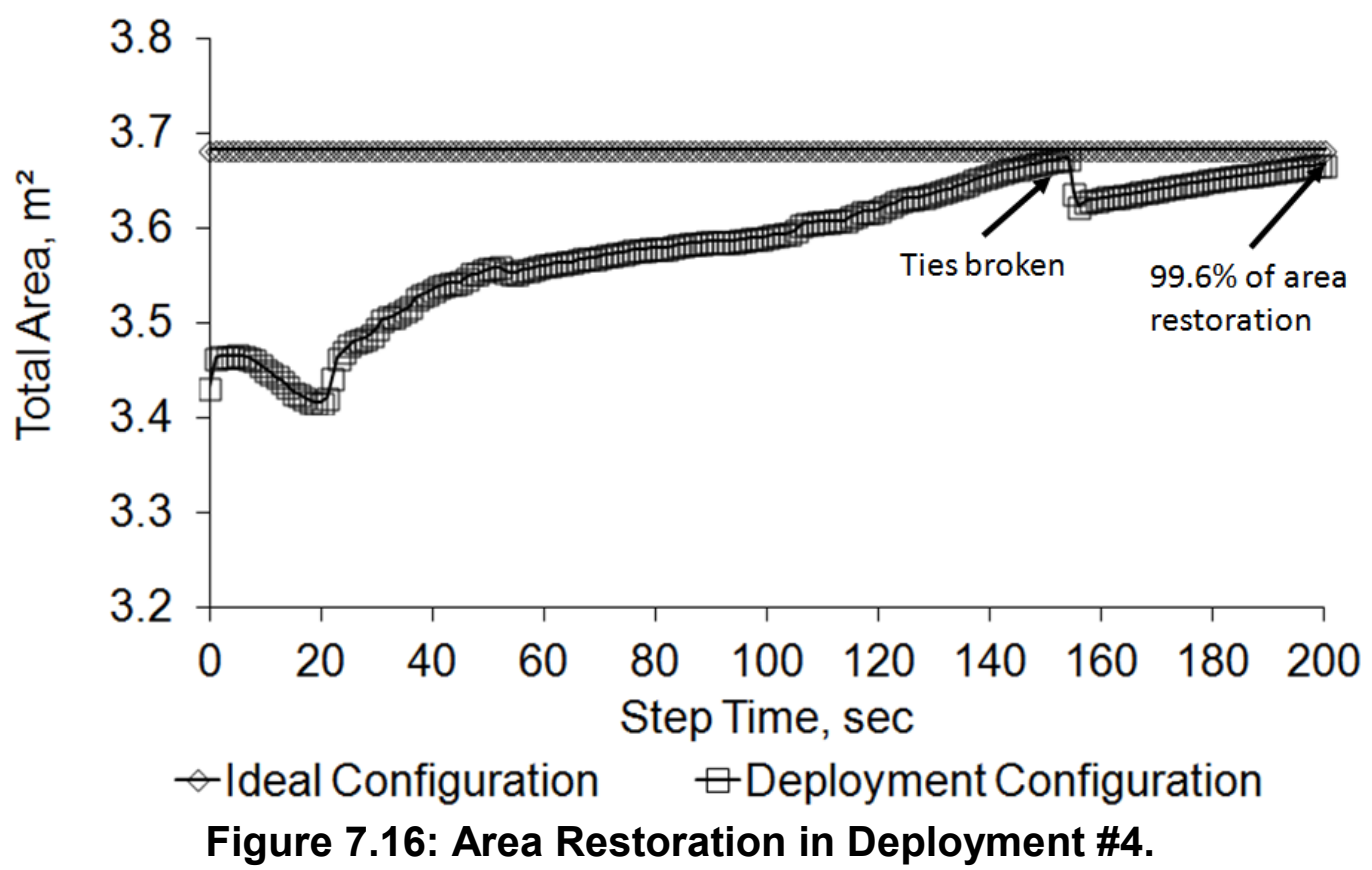

Figures 7.17 and 7.18 show the sequence of deployment for each case summarized in Table 7.3 compared to the experimental result. The air flow in Deployment \#1, \#2, and \#3 is not directed, therefore, the plug is inflated uniformly and instantaneously once the inflation part has been activated. The fluid exchange timing in Deployment \#4 has been specified to replicate the actual deployment behavior. However, the two half chambers along the $y-z$ plane (Figures 7.7, 7.8, and 7.9) limit the model from creating the smooth air flow characteristic discretely. From the deployment result of Deployment \#4, it is seen that the addition of more longitudinal chambers would help to replicate the actual deployment behavior even better. 


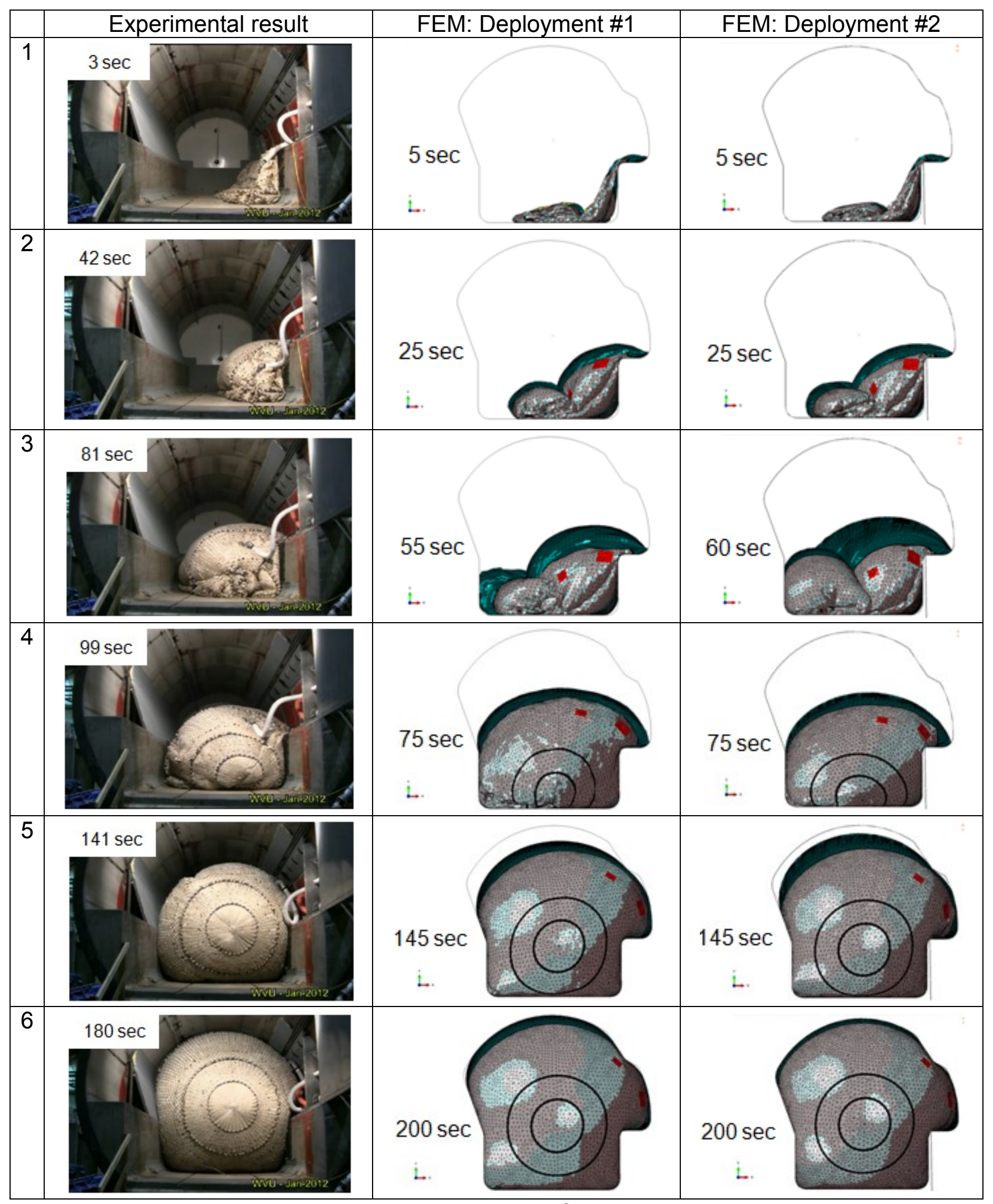

Figure 7.17: Deployment behavior of Deployment \#1 and \#2. 


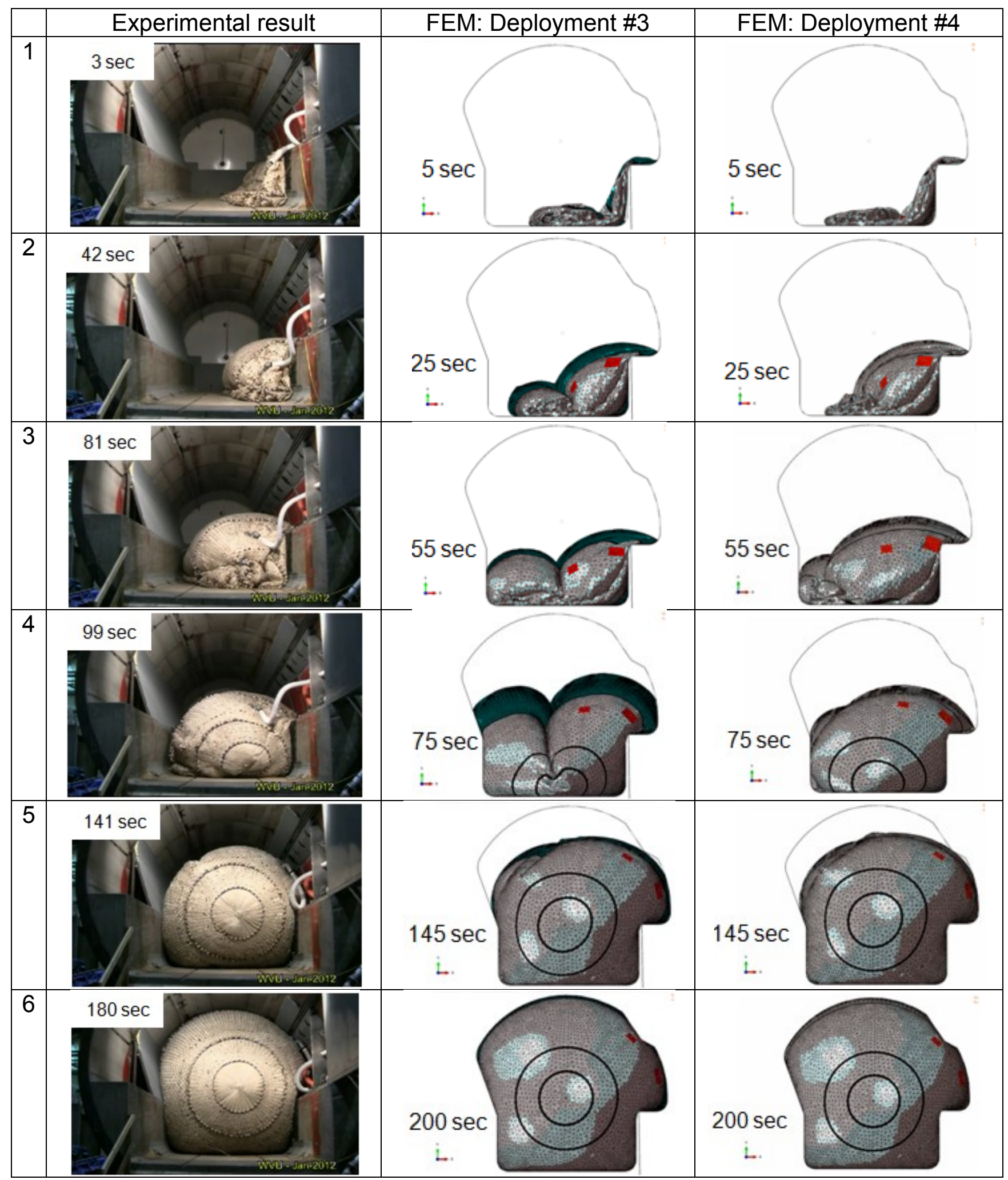

Figure 7.18: Deployment behavior of Deployment \#3 and \#4. 


\subsection{Summary}

Several factors needed to be identified and adjusted in order to build a complex model for Finite Element simulation of deployment of a full-scale inflatable structure within a tunnel segment. Such factors included definition of boundary conditions, direction of gravity force, relaxation, friction coefficients, material properties of equivalent fabric, inflation methodology, properties of fluid, and stiffness of tie used as a passive restraining mechanism. All these factors were described and implemented in a model of deployment of a folded inflatable structure.

The Uniform Pressure Method (UPM) was selected as the inflation algorithm due to its reduced computational cost. The multi-chamber and the fluid exchange approaches were needed to extend the capability of the UPM in order to replicate the actual deployment behavior obtained from experimental tests at full-scale.

The parameters of connector elements, representative of ties, were adjusted in order to reproduce a deployment behavior similar to what was observed experimentally. The two main reasons for using ties in the folded plug were:

- To drive material from the bottom to the upper zones of the tunnel during the deployment in order to improve the levels of local conformity.

- To have a sequential deployment in order to control the release of fabric material needed in critical areas of the tunnel such as corners and transitions.

The deployment case without connector elements has the lowest increase of contact surface area, the most bridging spots, and the most wrinkles and accumulation of material at the corner of floor among all the cases. Furthermore, the contact enhancement percentage increases nearly up to $34 \%$ when the plug leaves no bridges in critical corners after completion of the deployment and inflation.

The perimeter of the cylindrical portion of the plug was designed purposely to be oversized. This oversizing material was designed to be sufficient to take into account the potential manufacturing imperfections and unforeseen irregularities that the plug will have to conform around. The amount of oversizing material was kept constant at approximately $6 \%$ in this study.

The occurrence of wrinkles is a consequence of two main factors: a) the oversizing left in the perimeter of the cylindrical portion on the plug after complete 
deployment, and b) the ability of the inflation sequenced to drive and distribute membrane material uniformly around the contact perimeter. In this study, the location of wrinkles can be used to correlate the distribution and driving of the membrane material during the deployment. The location of wrinkles for Deployments \#1 and \#2 was accumulated at the bottom corner of the tunnel because the material was not driven up to the tunnel wall during the deployment. On the other hand, the location of wrinkles for Deployments \#3 and \#4 are observed at the wall of the tunnel because the passive restraining mechanism drove the oversizing material up to the ceiling and left minor wrinkles on the tunnel wall after the ties broke during the deployment.

Again, the Uniform Pressure Method resulted suitable to replicate the actual deployment behavior which correlated very well with the experimental results at relatively minimal computational cost. 


\section{Full-Scale Prototype: Evaluation of Axial Slippage}

\subsection{Introduction}

This chapter presents the results of axial slippage tests performed on models of the full-scale prototype pressurized under confined conditions. The objective of the work presented in this chapter is to evaluate the slippage resistance under various load combinations. The simulation results described in previous chapters, including deployment and equivalent material properties were used as input information for the models presented in this chapter.

The simulation of induced axial slippage for different values of friction coefficients provided an estimation of minimum values that need to be available in actual applications of the RTP concept.

\subsection{Induced Axial Slippage: Model Setup}

This section presents the minimum required parameters to create a model that can simulate an induced axial slippage test. The material properties of Material \#4 (see Section 3.8.2 of Chapter 3) are used to represent the equivalent fabric. The friction coefficient between plug and tunnel is 0.19 [59] and between plug and plug is 0.21 [60]. As mentioned in Section 7.7, the total area of membrane elements has been restored to 99.6\% after completion of the deployment and inflation stage, therefore the relaxation process can be neglected during slippage simulations. The mass scaling was not implemented in the slippage simulations as well since the scaled mass of model would affect slippage results. The results of Deployment \#4 presented in Chapter 7 have shown to have the best performance in terms of local conformity and maximum contact area, and therefore, they were selected as initial shape to perform the induced axial slippage simulations.

The inflated plug under confined conditions is pressurized by a fluid that can be either water or air depending on the loading scenario. As described in Chapter 2, the fluid cavity is modeled to represent the physical characteristics and calculate the volume 
of the selected fluid used for the simulations. Table 8.1 displays the physical properties of water and air used in the simulations.

Table 8.1: Physical properties of water and air.

\begin{tabular}{|c|c|c|c|}
\hline \multicolumn{2}{|c|}{ Material Propeties of Fluid } & $\begin{array}{c}\text { Water at } 277.15 \mathrm{~K} \\
\left(4^{\circ} \mathrm{C}, 39.2^{\circ} \mathrm{F}\right)\end{array}$ & $\begin{array}{c}\text { Air at } 293.15 \mathrm{~K} \\
\left(20^{\circ} \mathrm{C}, 68^{\circ} \mathrm{F}\right)\end{array}$ \\
\hline \multirow{3}{*}{ Fluid Density } & $\mathrm{kg} / \mathrm{m}^{3}$ & 1000 & 1.205 \\
\cline { 2 - 4 } & $\mathrm{lbm} / \mathrm{in}^{3}$ & $3.612 \mathrm{E}-2$ & $4.3337 \mathrm{E}-5$ \\
\cline { 2 - 4 } & $\frac{l \mathrm{lbf} \cdot \mathrm{sec}^{2}}{i n^{4}}$ & $0.94 \mathrm{E}-4$ & $1.123 \mathrm{E}-7$ \\
\hline \multirow{2}{*}{$\begin{array}{c}\text { Fluid Bulk Modulus } \\
\mathrm{N} / \mathrm{m}^{2}\end{array}$} & $2.15 \mathrm{E} 9$ & $1.42 \mathrm{E} 5$ (Adiabatic) \\
\hline $\begin{array}{c}\text { Fluid Expansion } \\
\text { Coefficient }\end{array}$ & $\mathrm{lbf} / \mathrm{in}^{2}$ & $3.12 \mathrm{E} 5$ & 14.6488 \\
\hline
\end{tabular}

The required internal $(\mathrm{Pi})$ and external $(\mathrm{Pe})$ pressures used for the simulations are $0.1172 \mathrm{MPa}(17 \mathrm{psi})$ and $0.0797 \mathrm{MPa}(11.56 \mathrm{psi})$, respectively. The induced axial slippage tests are modeled for two different conditions:

I. Normal operating conditions at required pressures

II. Assuming depressurization of the plug

The pressurization event for normal operating conditions at required pressures is illustrated in Figure 8.1. For this condition, the internal pressure $(\mathrm{Pi})$ increases until it reaches the maximum required pressure at the $1^{\text {st }}$ second and then remains constant for the rest of the simulation. On the other hand, the external pressure $(\mathrm{Pe})$ is applied on the rear hemispherical cap, as schematically illustrated in Figure 8.2, at the $4^{\text {th }}$ second, then reaches the maximum design pressure at the $8^{\text {th }}$ second, and remains constant for the rest of the simulation. 


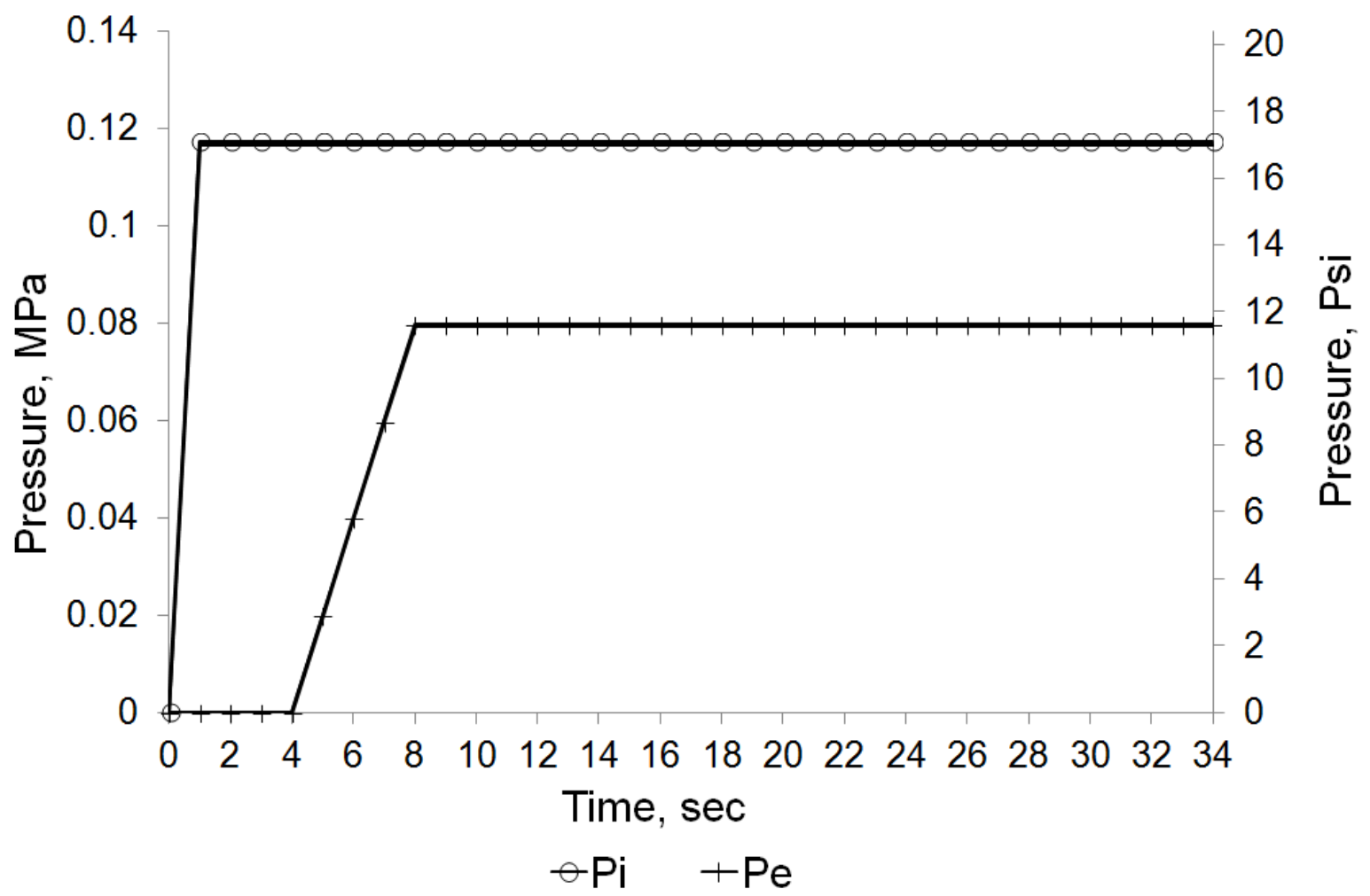

Figure 8.1: Pressurization event for normal operating conditions

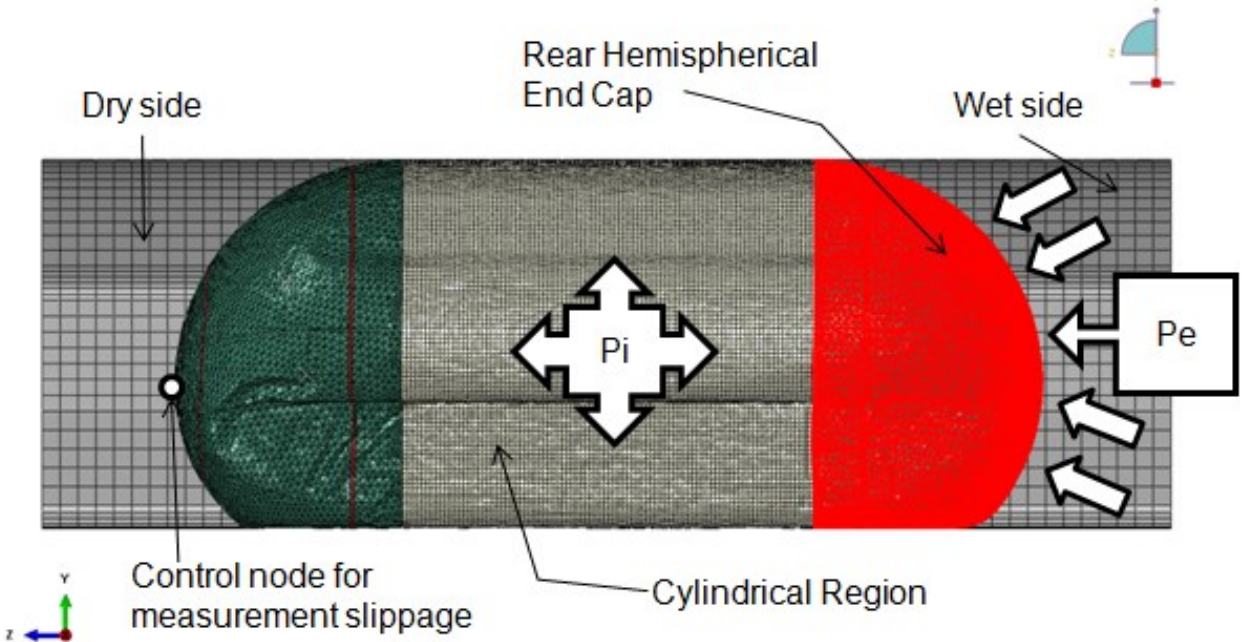

Figure 8.2: Pressurization Scheme for Internal and External Pressures.

The sequence of pressurization and depressurization of the plug to induce slippage is illustrated in Figure 8.3. The pressure $\mathrm{Pi}$ is applied to the entire internal surface of the plug from zero pressure until it reaches the maximum inflation pressure at the $1^{\text {st }}$ second and then remains constant for a certain period of time. The external 
pressure $(\mathrm{Pe})$ is then applied on the rear hemispherical end cap at the $4^{\text {th }}$ second and reaches its maximum at the $8^{\text {th }}$ second and then remains constant for the rest of the simulation. The scenario of plug depressurization (for example, due to a leakage in the fabric), starts at the $12^{\text {th }}$ second with the internal pressure reducing at 0.001875 $\mathrm{MPa} / \mathrm{sec}$ gradually until it finally matches the external pressure at the $32^{\text {nd }}$ second. Then, both internal and external pressures remain constant until the plug slips or not, depending the friction coefficient assigned to the system.

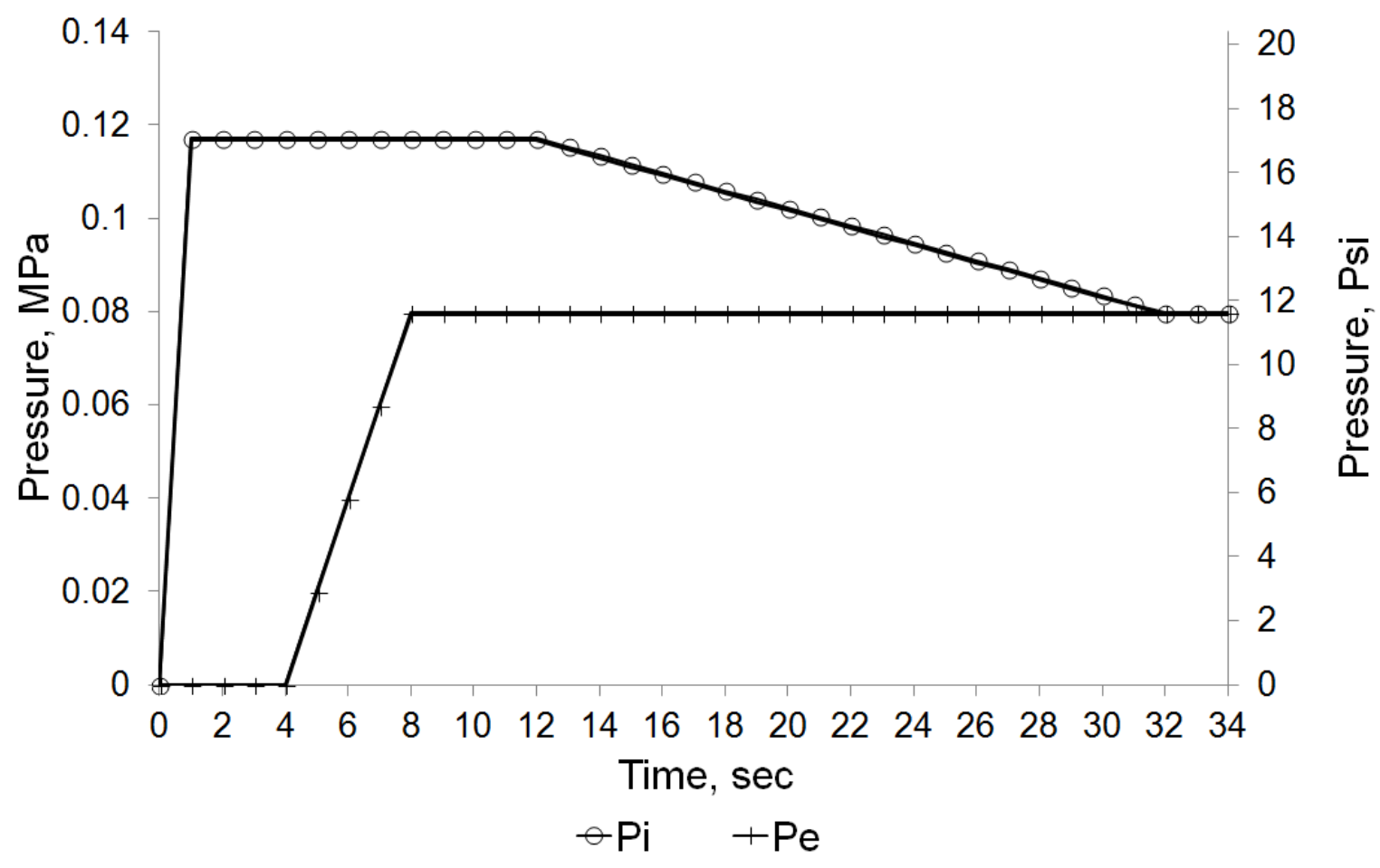

Figure 8.3: Sequence of pressurization and depressurization to induce slippage of the plug.

Four loading scenarios were simulated following the pressurization sequence shown in Figure 8.3. Each one included combinations of fluid properties and different friction coefficients. The internal or plug pressure is applied in two ways: 1) A uniform pressure is assumed to be produced by compressible gas (air) that occupies the volume of the plug; 2) A hydrostatic pressure is assumed to be generated by water contained within the plug. The external pressure $(\mathrm{Pe})$ also follows either a uniform or a hydrostatic distribution applied toward the external surface of the membrane in the rear 
hemispherical end-cap. The details of the pressure distribution were presented in Chapter 2.

The loading scenarios are summarized in Table 8.2. Loading scenario \#1 includes internal pressure as hydrostatic load with physical properties of water and external pressure as hydrostatic load. This loading scenario is the testing scenario that was implemented experimentally at WVU [58]. Loading scenario \#2 assumes internal pressure as uniform pressure with physical properties of air and external pressure as hydrostatic load. Loading scenarios \#1 and \#2 are feasible field scenarios. Loading scenarios \#3 and \#4 are unlikely field scenarios but included to complete the combinations of the modeling plan. These last two scenarios are assumed to have uniform pressure applied on the rear hemispherical end-cap of the plug, while an internal hydrostatic and a uniform pressure are applied with water and air, respectively, within the plug during the simulations.

Table 8.2: Loading Scenarios for Induced Slippage.

\begin{tabular}{|l|l|l|}
\hline & Pe (Hydrostatic) & Pe (Uniform) \\
\hline Pi (Hydrostatic) & (\#1) HydroPi-HydroPe & (\#3) HydroPi-UniPe \\
\hline Pi (Uniform) & (\#2) UniPi-HydroPe & (\#4) UniPi-UniPe \\
\hline
\end{tabular}

Figure 8.4 shows an overview of the model for loading scenarios \#1 and \#3. The water within the plug was represented with solid elements and analyzed with the same approach presented in Section 3.7.3. The model of water in the full-scale plug contains $86.8 \%$ of the internal volume of the plug, therefore an equivalent density of water needs to be implemented. The equivalent density of solid elements for water is $1152 \mathrm{~kg} / \mathrm{m}^{3}$ $\left(0.04165 \mathrm{lbm} / \mathrm{in}^{3}\right.$ or $\left.1.079 \mathrm{E}-4 \mathrm{lbf} . \mathrm{sec}^{2} / \mathrm{in}^{4}\right)$. For scenarios \#2 and \#4, the mass of air (149 $\mathrm{kg}$ or $328.52 \mathrm{lbm}$ ) is added to the membrane elements of plug assuming the plug carries the mass of air during slippage simulation. Figure 8.5 illustrates a contour of hydrostatic pressure distribution for loading scenario \#1 where the maximum required pressures of $\mathrm{Pi}=0.117 \mathrm{MPa}(17 \mathrm{psi})$ and $\mathrm{Pe}=0.0797 \mathrm{MPa}(11.56 \mathrm{psi})$ measured at the tunnel floor level. Appendix $\mathrm{E}$ demonstrates detailed calculations of equivalent densities of water and air. 


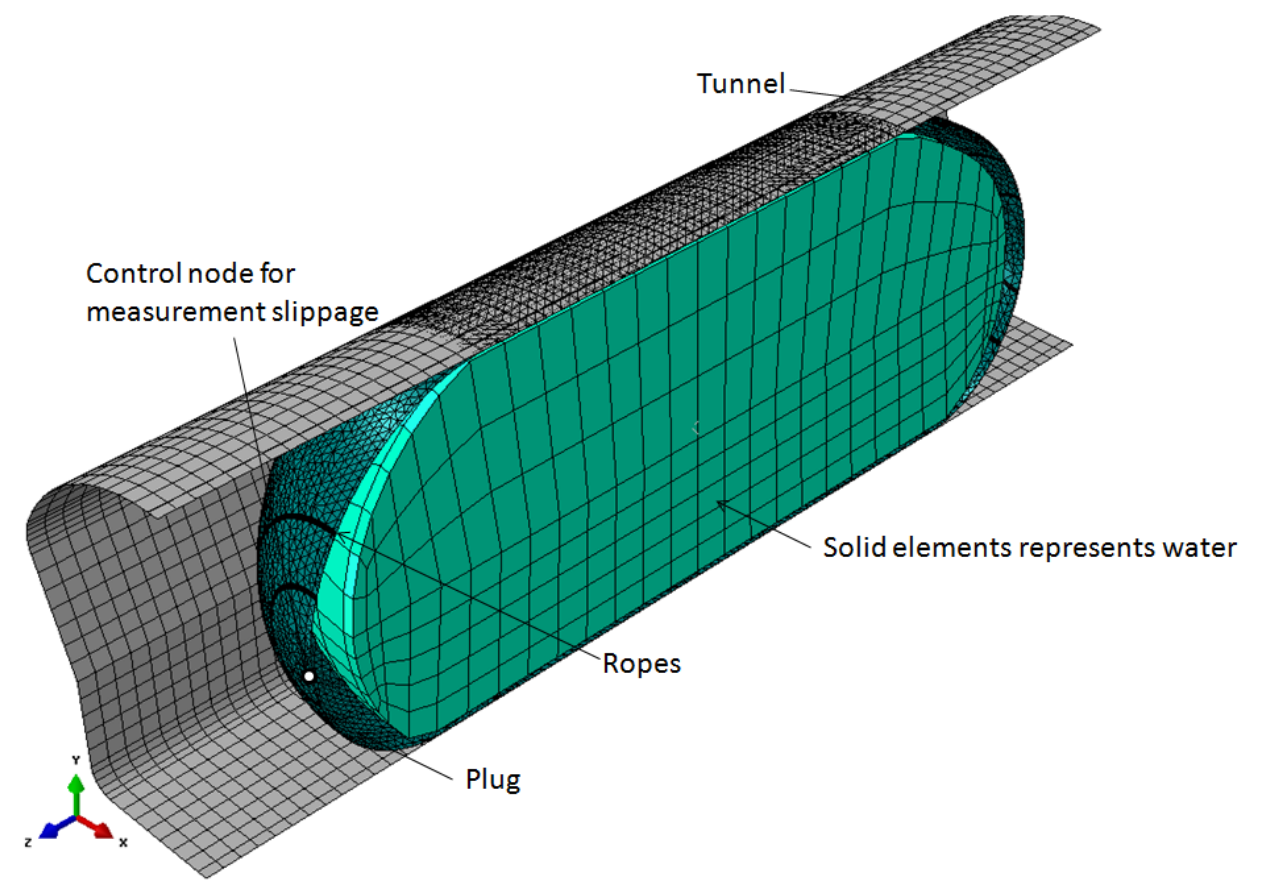

Figure 8.4: Cut view of FE model setup for slippage scenarios \#1 and \#2.

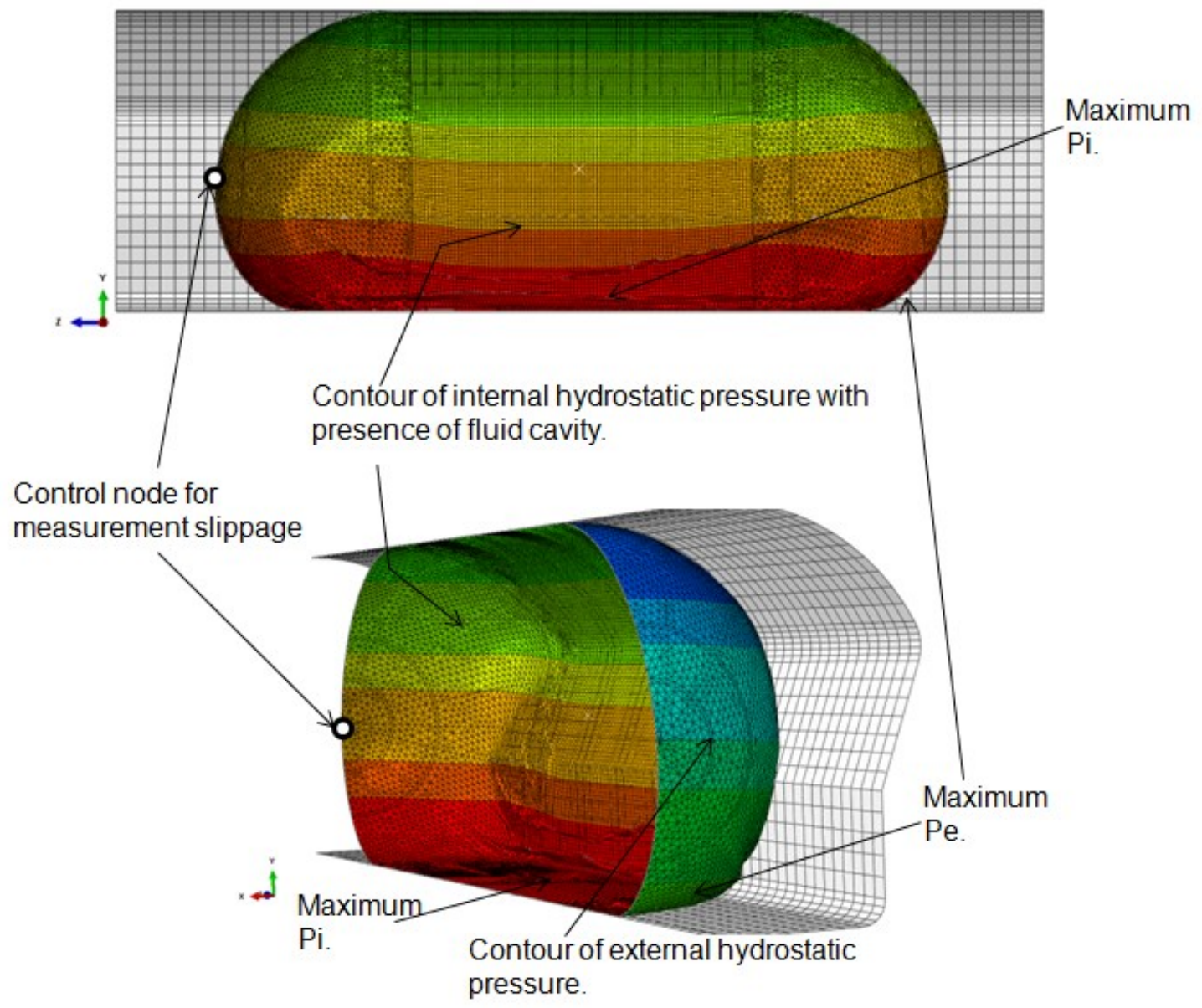

Figure 8.5: Cut view of Loading Scenario \#1 with hidden solid elements of fluid 
Table 8.3 summarizes the complete FE simulation plan including pressurization events, loading scenarios, and the friction coefficients (the contact interaction behavior between plug and tunnel). The friction coefficient between plug and tunnel of 0.19 was obtained from experimental results [59] and it is used in every simulation case. Loading Scenarios \#1 and \#2 were tested with four values of friction coefficient. That is a total of 20 simulations.

Table 8.3: Complete Induced Slippage FE Simulation Plan

\begin{tabular}{|c|c|c|c|c|c|c|}
\hline $\begin{array}{l}\text { Pressurization } \\
\text { Events }\end{array}$ & $\begin{array}{l}\text { Group } \\
\text { Number }\end{array}$ & Loading Scenarios & \multicolumn{4}{|c|}{ Friction Coefficients } \\
\hline \multirow{4}{*}{$\begin{array}{c}\text { Normal } \\
\text { Operating } \\
\text { Conditions }\end{array}$} & 1 & (\#1) HydroPi-HydroPe & 0.13 & 0.15 & 0.19 & 0.25 \\
\hline & 2 & (\#2) UniPi-HydroPe & 0.13 & 0.15 & 0.19 & 0.25 \\
\hline & 3 & (\#3) HydroPi-UniPe & - & - & 0.19 & - \\
\hline & 4 & (\#4) UniPi-UniPe & - & - & 0.19 & - \\
\hline \multirow{4}{*}{$\begin{array}{l}\text { Assuming } \\
\text { Depressurization } \\
\text { of the Plug }\end{array}$} & 5 & (\#1) HydroPi-HydroPe & 0.13 & 0.15 & 0.19 & 0.25 \\
\hline & 6 & (\#2) UniPi-HydroPe & 0.13 & 0.15 & 0.19 & 0.25 \\
\hline & 7 & (\#3) HydroPi-UniPe & - & - & 0.19 & - \\
\hline & 8 & (\#4) UniPi-UniPe & & - & 0.19 & - \\
\hline
\end{tabular}

\subsection{Simulation Results}

\subsubsection{Results for Normal Operating Conditions}

This section presents the results for the four groups under the pressurization event of normal operating conditions summarized in Table 8.3. Figure 8.6 illustrates the slippage simulation result of Group 1 . The plot shows a relatively little slippage in the range of $0.01 \mathrm{~m}$ to $0.07 \mathrm{~m}$ for friction coefficients in the range of 0.13 to 0.25 . This set of results suggests that the friction coefficient between plug and tunnel has to be at least larger than 0.15 to maintain the system stable. The relatively small slip observed at $10^{\text {th }}$ second in the model HydroPi-HydroPe-f013 is attributed to the gradual increase of external pressure from zero to its maximum constant value. 


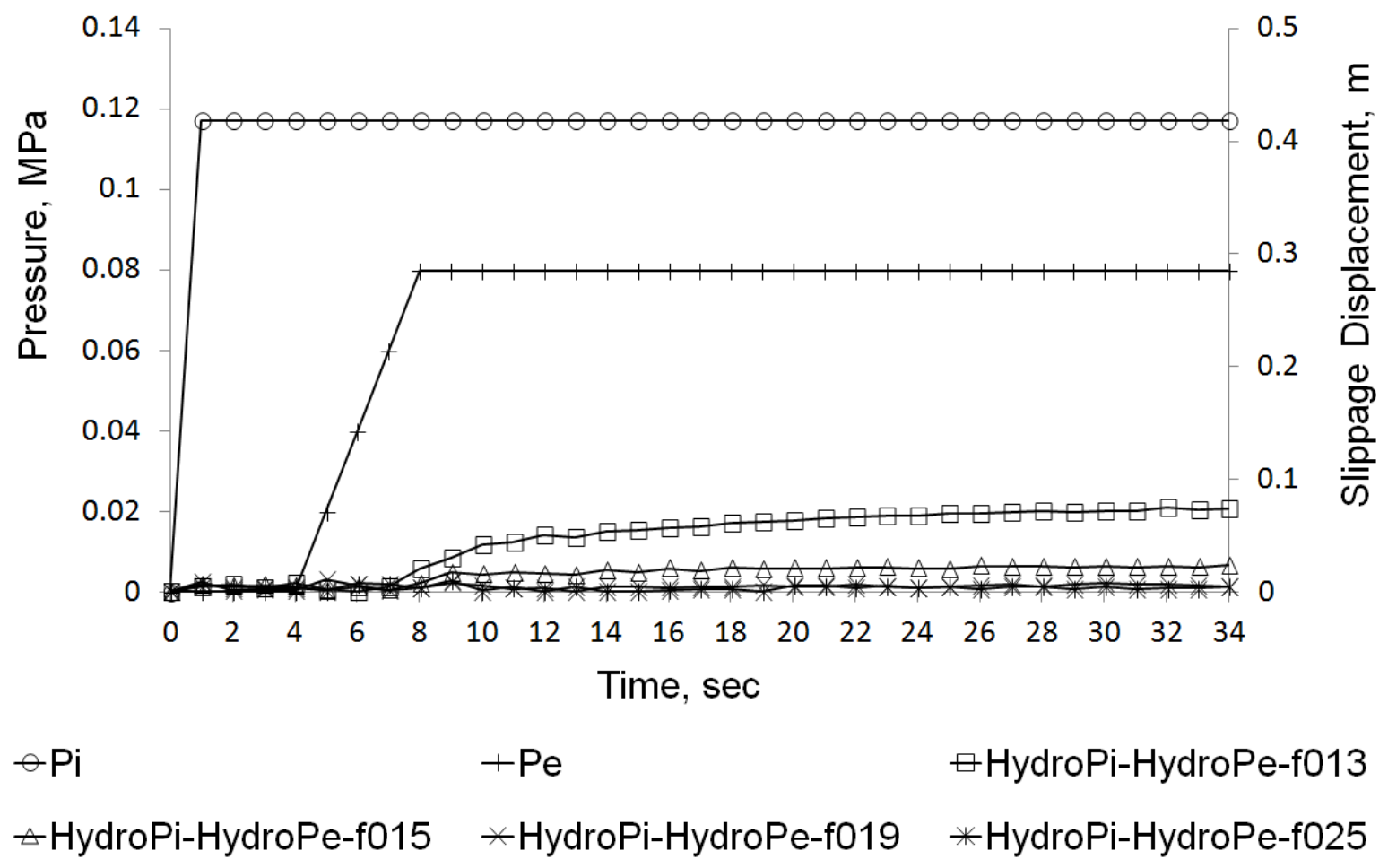

Figure 8.6: Slippage results for Group 1

Figure 8.7 shows the slippage results of Group 2 (UniPi-HydroPe). In this case, there is no obvious slippage except for a friction coefficient of 0.13 for which the plug slipped significantly less than in Group 1. Comparing the slippage distances illustrated in Figure 8.6 and Figure 8.7 on the model with friction coefficient 0.13 , a uniform internal pressure seems to hold the plug better than a hydrostatic internal pressure. Again, a friction coefficient of at least 0.15 seems to be the minimum value to maintain the plug axially stable. 


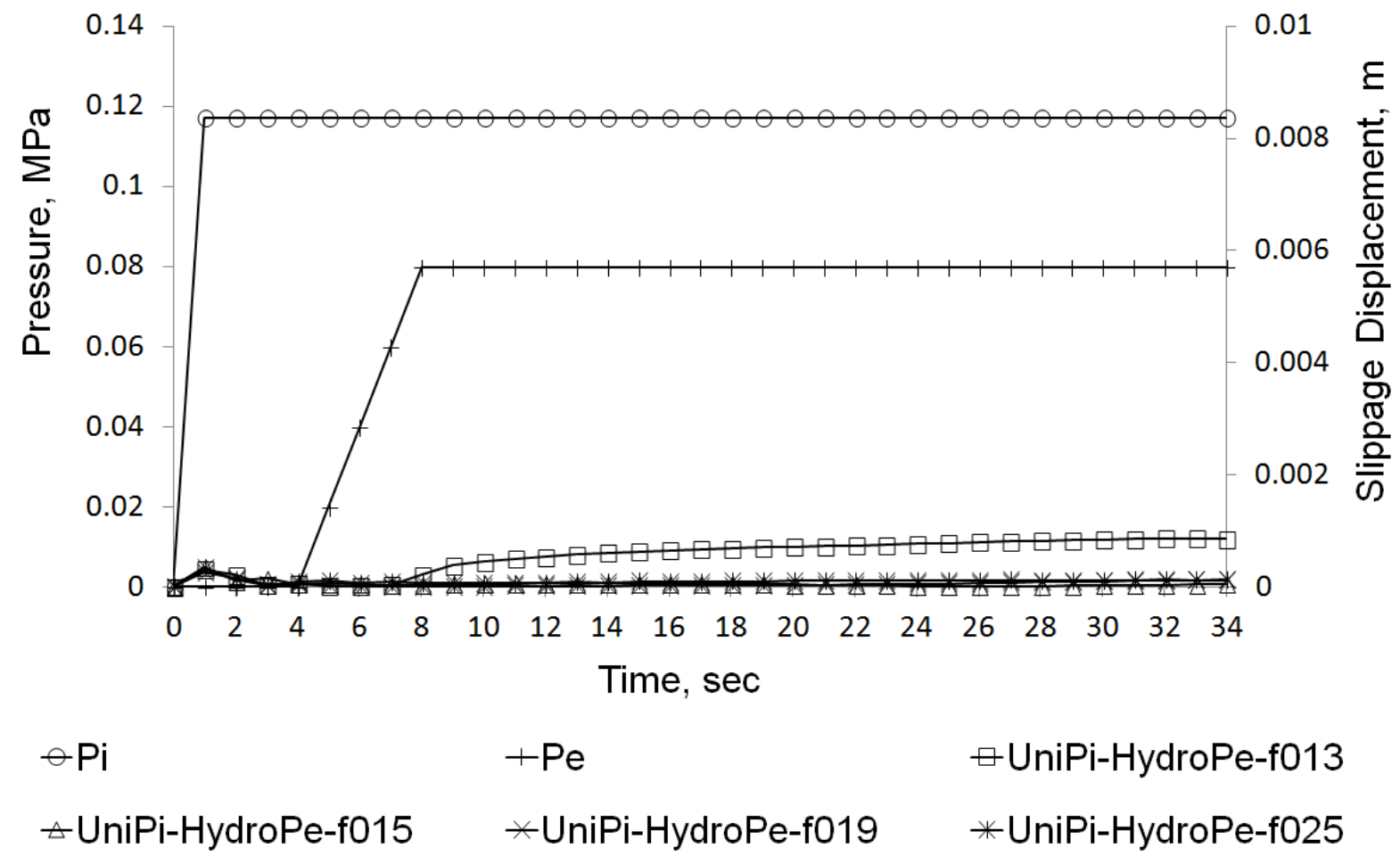

Figure 8.7: Slippage results for Group 2.

The slippage results for Group 3 are shown in Figure 8.8. The UniPi-UniPe scenario assumes that the plug is pressurized by a uniform internal pressure and also a uniform pressure acts on the external surface of the rear hemispherical end of the plug. Only one friction coefficient was tested (0.19) and the results show that the plug remained stable for this loading scenario.

Figure 8.9 shows the slippage result of Group 4. Only a friction coefficient of 0.19 was tested. The plug slipped approximately $0.10 \mathrm{~m}$ before reaching a new equilibrium position for the loading scenario of HydroPi-UniPe. This result suggests that an external uniform pressure is more severe than one with hydrostatic distribution. For this case, a friction coefficient higher than 0.19 would be needed to maintain the plug in place. 


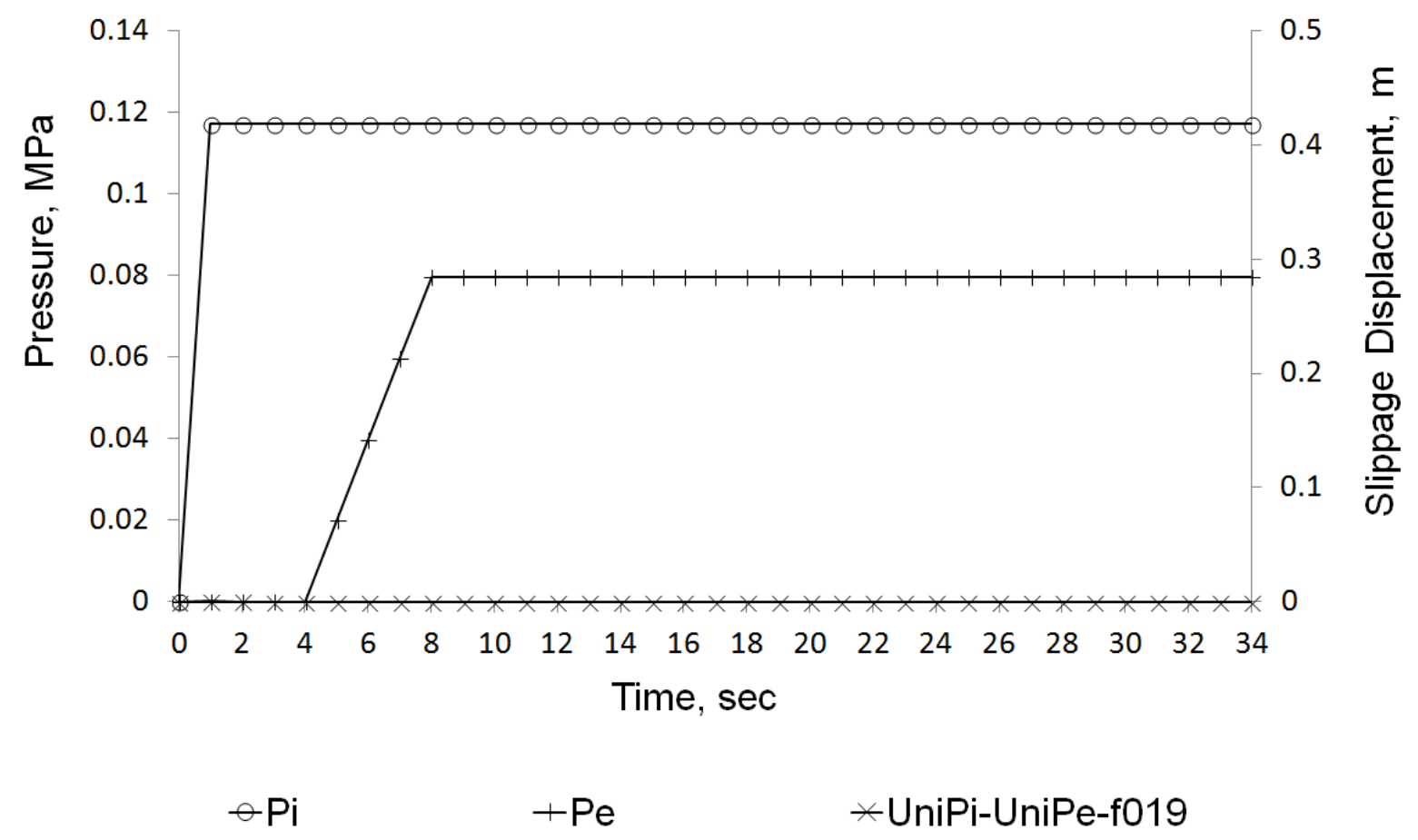

Figure 8.8: Slippage results for Group 3.

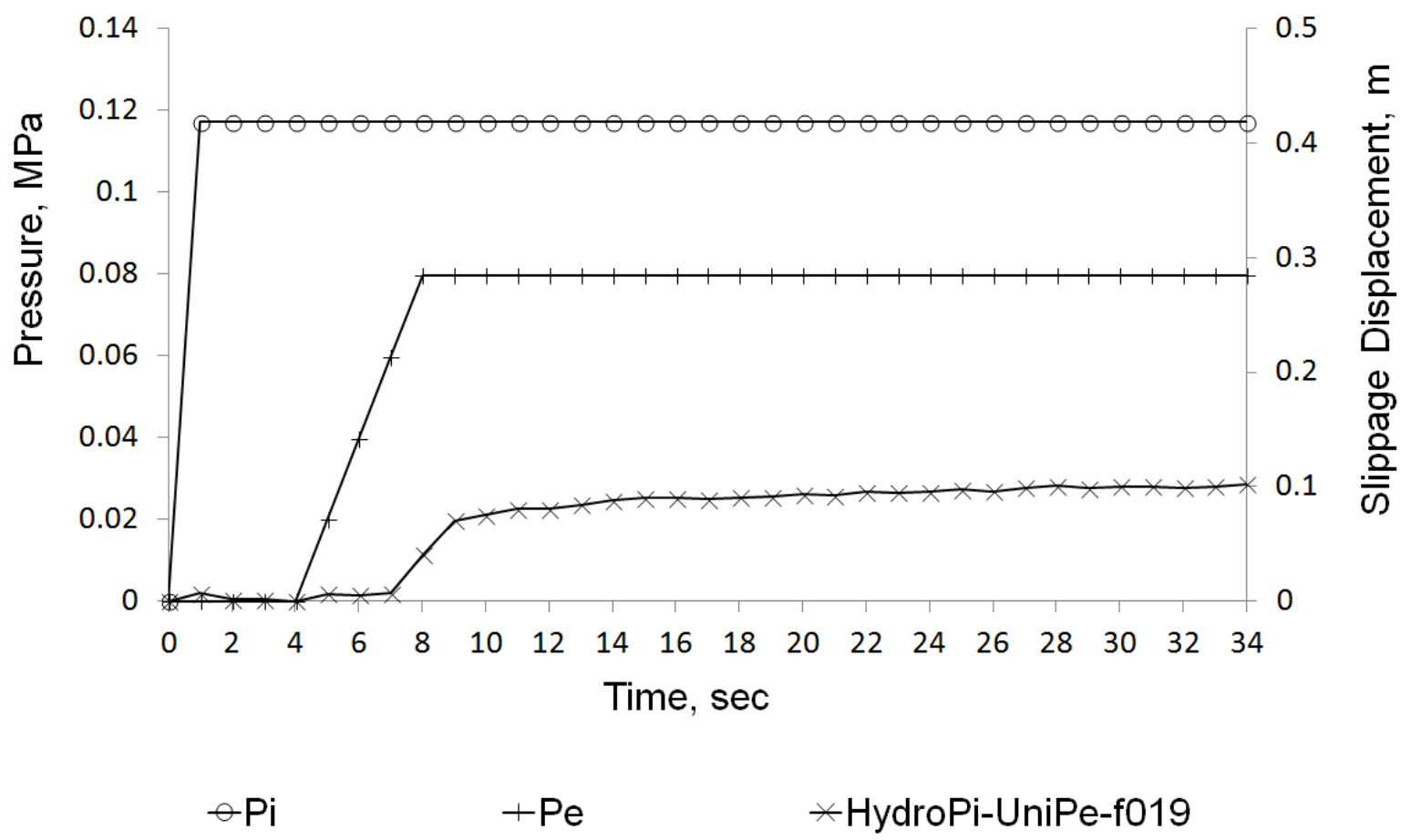

Figure 8.9: Slippage results for Group 4. 


\subsubsection{Results for Assuming Depressurization of the Plug}

This section presents the results of a set of simulations that reproduce the effect of the friction coefficient on the axial stability when there is a gradual depressurization of the plug. Results for Groups 5 to 8 (Table 8.3) are discussed here. The holding resistance ratio $(\mathrm{Pe} / \mathrm{Pi})$ introduced in Chapter 3 is again used here to identify how close the external and internal pressures are to each other at the instant of slippage.

Figure 8.10 summarizes the slippage results of Group 5 (HydroPi-HydroPe). In this group, the internal surface of the plug was subjected to hydrostatic pressure with fluid cavity (described in Chapter 2, in which the fluid has mass and the characteristics of water) and the external surface of rear plug end cap has hydrostatic pressure as well. Results show that, among the models, the model with a friction coefficient of 0.25 has the least slippage distance (less than $0.1 \mathrm{~m}$ ). Models with friction coefficients of 0.10 , 0.13 , and 0.15 basically slipped away from tunnel and the plug was barely held with a friction coefficient 0.19 . When the internal and external pressures were almost equivalent.

Table 8.4 illustrates the influence of friction coefficient on the holding resistance defined as the ratio $\mathrm{Pe} / \mathrm{Pi}$. For a constant $\mathrm{Pe}$ and a decreasing $\mathrm{Pi}$, the ratio $\mathrm{Pe} / \mathrm{Pi}$ provides an indication on how stable is the system for a given friction coefficient. A model without a holding resistance value means that the slippage occurred before the depressurization happened and almost immediately after the beginning of the action of $\mathrm{Pe}$. On the other hand, a model that has holding resistance coefficient of 1.00 indicates that the internal pressure, $\mathrm{Pi}$, dropped to the same level as the external pressure, $\mathrm{Pe}$, and the system can slip, or not, depending of the friction coefficient. The system can also reach a new equilibrium position as in the case of $\mu=0.25$ illustrated in Figure 8.10. The values of holding resistance show that the minimum friction coefficient between plug and tunnel, in a event of plug depressurization at a ratio of $0.001875 \mathrm{MPa} / \mathrm{sec}$, has to be higher than 0.25 to prevent the plug slipping completely away from the tunnel. 


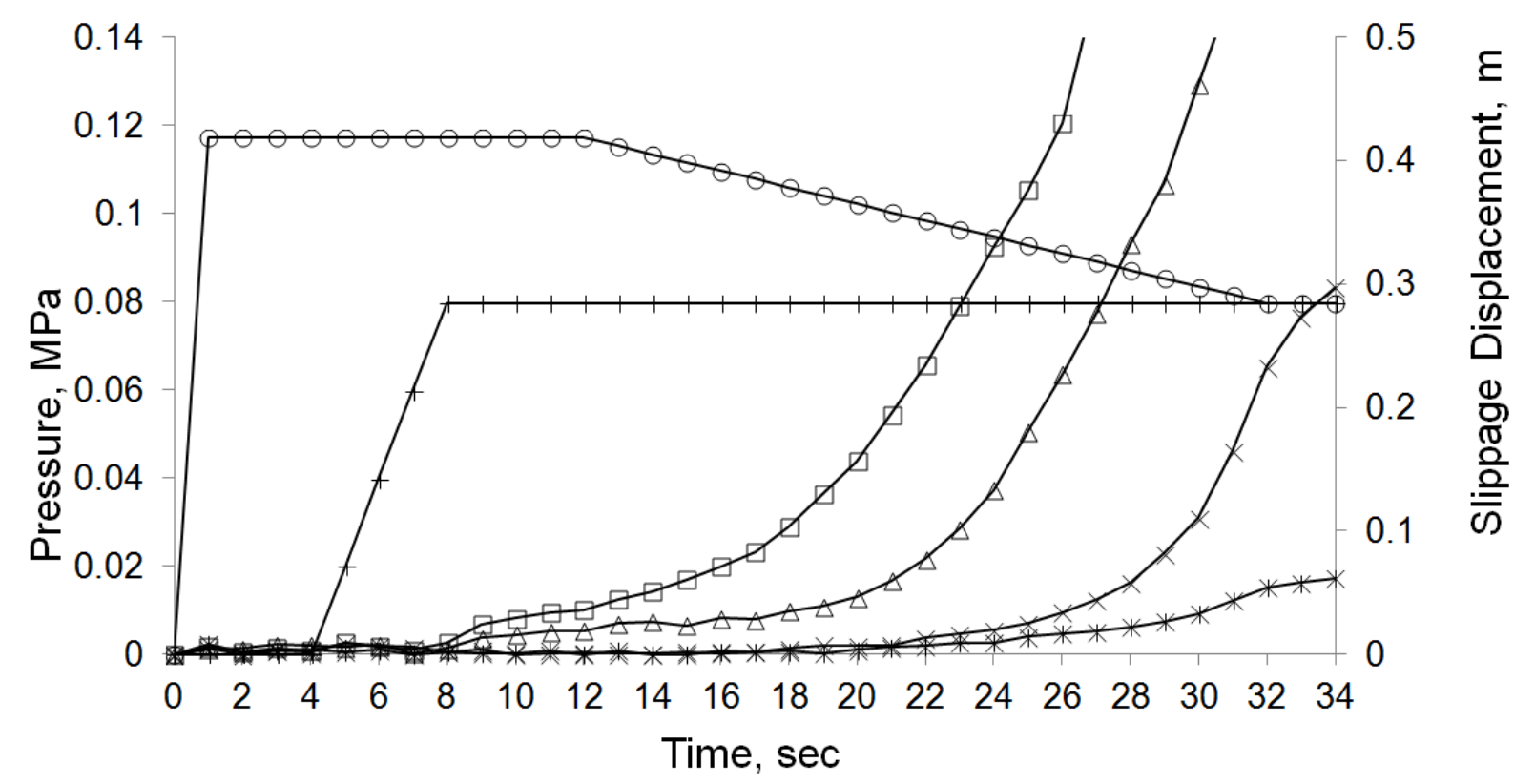

$\ominus \mathrm{Pi} \quad+\mathrm{Pe} \quad \square$ HydroPi-HydroPe-f013

$\triangle$ HydroPi-HydroPe-f015 *HydroPi-HydroPe-f019 *HydroPi-HydroPe-f025

Figure 8.10: Slippage results for Group 5.

Table 8.4: Influence of Friction Coefficient on Holding Resistance Ratio.

\begin{tabular}{|c|c|c|c|}
\hline Group & Pressurization Event & Friction Coefficient, $\mu$ & Pe/Pi ratio reached at slippage \\
\hline \multirow{3}{*}{5} & \multirow{3}{*}{5} & 0.13 & 0.77 \\
\cline { 3 - 4 } & \multirow{2}{*}{\begin{tabular}{c} 
Assuming \\
Depsurization of \\
\cline { 3 - 4 }
\end{tabular}} & 0.15 & 0.84 \\
\cline { 3 - 4 } & & 0.19 & 0.95 \\
\cline { 3 - 4 } & & 0.25 & 1.00 \\
\hline
\end{tabular}

Figure 8.11 shows sequences of slippage for each case of Group 5. The pressurization sequence illustrated in Figure 8.10 can be divided into six stages:

- Stage 1: At the $1^{\text {st }}$ second, the internal pressure is at its maximum value.

- Stage 2: At the $8^{\text {th }}$ second, both internal and external pressures reached their maximum values.

- Stage 3: At the $12^{\text {th }}$ second, depressurization begins.

- Stage 4: At the $22^{\text {nd }}$ second, depressurization has proceeded through half way. 
- Stage 5: At the $32^{\text {nd }}$ second, depressurization ended (internal pressure $=$ external pressure).

- Stage 6: At the $34^{\text {th }}$ second, the simulation ends.

Figures 8.11 and 8.12 also shows sequences of slippage for the cases of Group 5 for various friction coefficients. Note that, the model with friction coefficient of 0.13 in Figure 8.11 shows slipping phenomenon when the depressurization has proceeded from Stage 3 to Stage 4.

The model with friction coefficient of 0.15 in Figure 8.11 shows slipping phenomenon in between Stage 4 and 5. As described previously, the models with $\mu=$ 0.19 (Figure 8.12) shows slipping phenomenon almost at the end of Stage 5 (at $30^{\text {th }}$ second), while the model with $\mu=0.25$ (Figure 8.12 ) slightly slipped when the internal pressure nearly reached the same magnitude as the external pressure.

In Figure 8.11, note that at the instant of slippage (stage 5 and 6 ) of models with $\mu=0.13$ and 0.15 the plug shows a tendency to lose contact in the upper part of the tunnel which in practical terms would mean that the plug has lost its capacity of scaling the tunnel section and is no longer stable. In the models with $\mu=0.19$ and 0.25 , the overall plug shape remains practically unchanged until stage 5 after which there is clear tendency to lose contact in the upper portion of the plug as illustrated in Figure 8.12. 


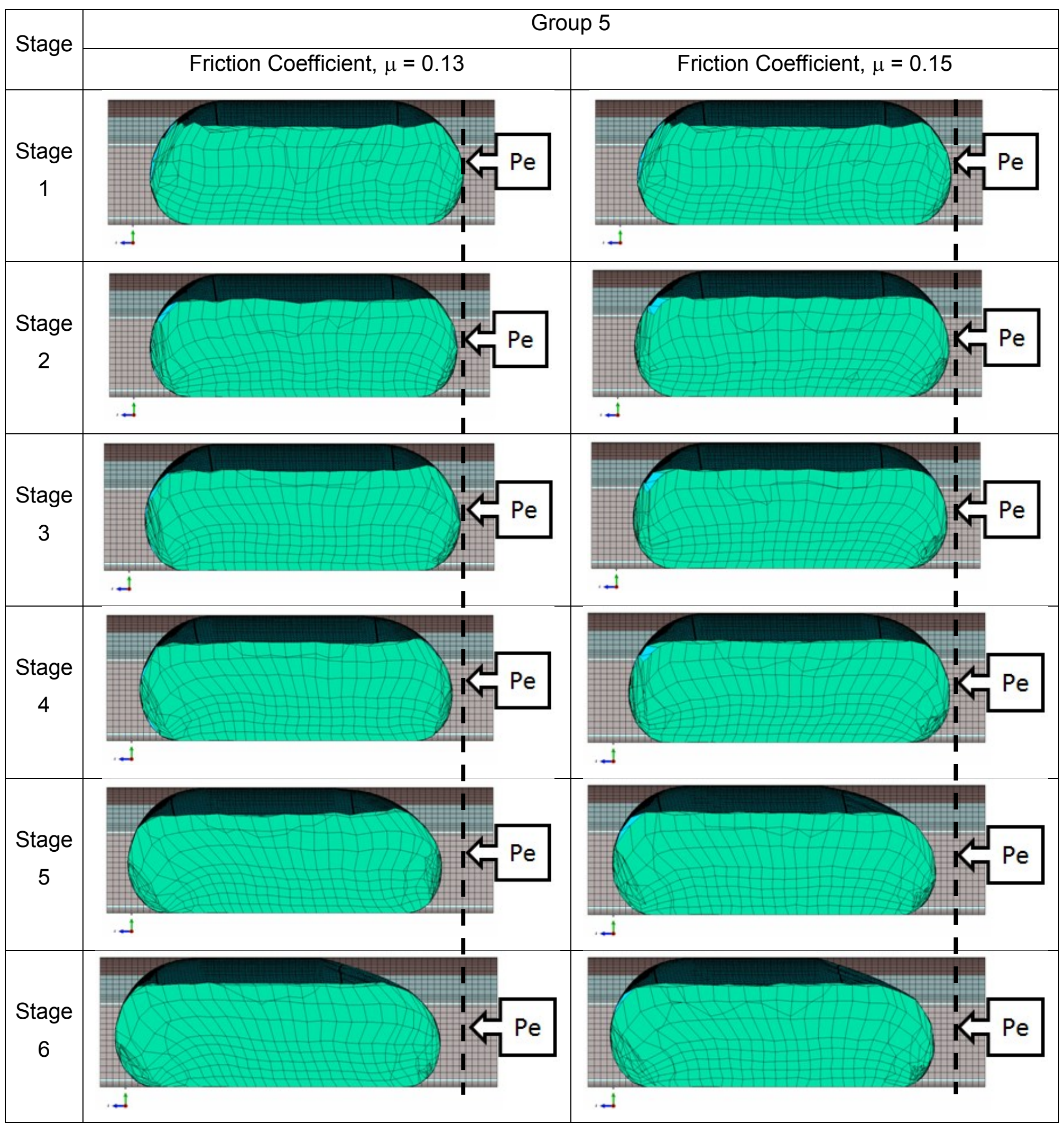

Figure 8.11: Sequence of slippage for friction coefficients of 0.13 and 0.15

(Deformation scale factor $=1$ ). 


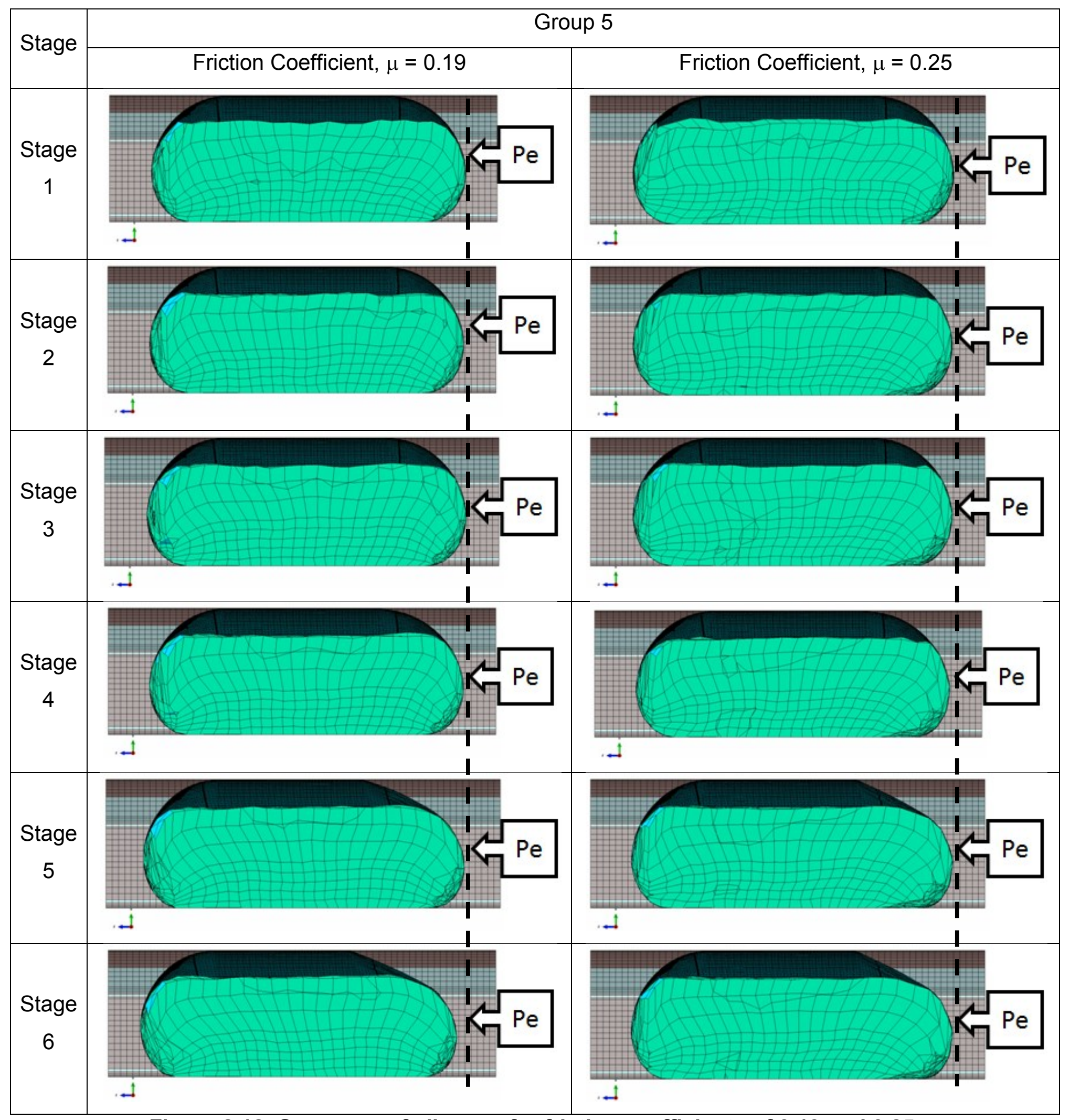

Figure 8.12: Sequence of slippage for friction coefficients of 0.19 and 0.25 .

(Deformation scale factor $=1$ ). 
Figure 8.13 shows the slippage results of Group 6 (UniPi-HydroPe). In this group, the internal surface of plug has a uniform pressure with fluid cavity (described in Chapter 2) and the external surface of rear plug end-cap has a hydrostatic pressure.

Results show that the models with friction coefficients of 0.19 and 0.25 have the least slippage displacement (less than $0.01 \mathrm{~m}$ ) among all the models. Model with friction coefficient of 0.13 slipped away from tunnel. The model with friction coefficient of 0.15 displaced for less than $0.10 \mathrm{~m}$ before reaching a new equilibrium position. Note that the model with friction coefficient of 0.13 held longer than the similar case shown for Group 5 and shows the affect of having uniform pressure versus hydrostatic pressure in the plug.

Table 8.5 summarizes the influence of friction coefficient the holding resistance of the cases corresponding to Group 6 . The models with friction coefficients of 0.13 , and 0.15 have holding resistances of 0.96 , and 0.98 , respectively. Models with friction coefficients of 0.19 and 0.25 have reached a holding resistance ratio of 1.0 without slipping indicating that the plug would still have holding resistance to remain stable.

The graphic position of the plug for the different cases simulated in Group 6 is shown in Figures 8.14 and 8.15. These figures show the sequence of slippage results corresponding to models with friction coefficients of $0.13,0.15,0.19$, and 0.25 . As seen in the pictures, models with friction coefficients of 0.13 , and 0.15 slipped in between Stage 4 and Stage 5. The models with friction coefficients of 0.19 and 0.25 did not slip and remained stable during the entire simulation.

From Figure 8.14 and 8.15 is worth to note the plug shape at stage 5 and 6 when $\mathrm{Pe}$ and $\mathrm{Pi}$ are equal. Contrary to what is seen in the cases of Group 5, the tendency of the plug to lose contact is at the tunnel floor level rather than from the upper part. This is attributed to the characteristics of the distribution of pressures. A uniform pressure inside the plug can be achieved by compressing a gas (air in this case), while the hydrostatic external pressure is achieved with water. At the critical stage of having both pressures equal, the maximum external pressure is at the tunnel floor level; because of 
the compressibility of air, the rear end-cap will tend to uplift to follow the hydrostatic external pressure distribution as seen in the different cases of Group 6.

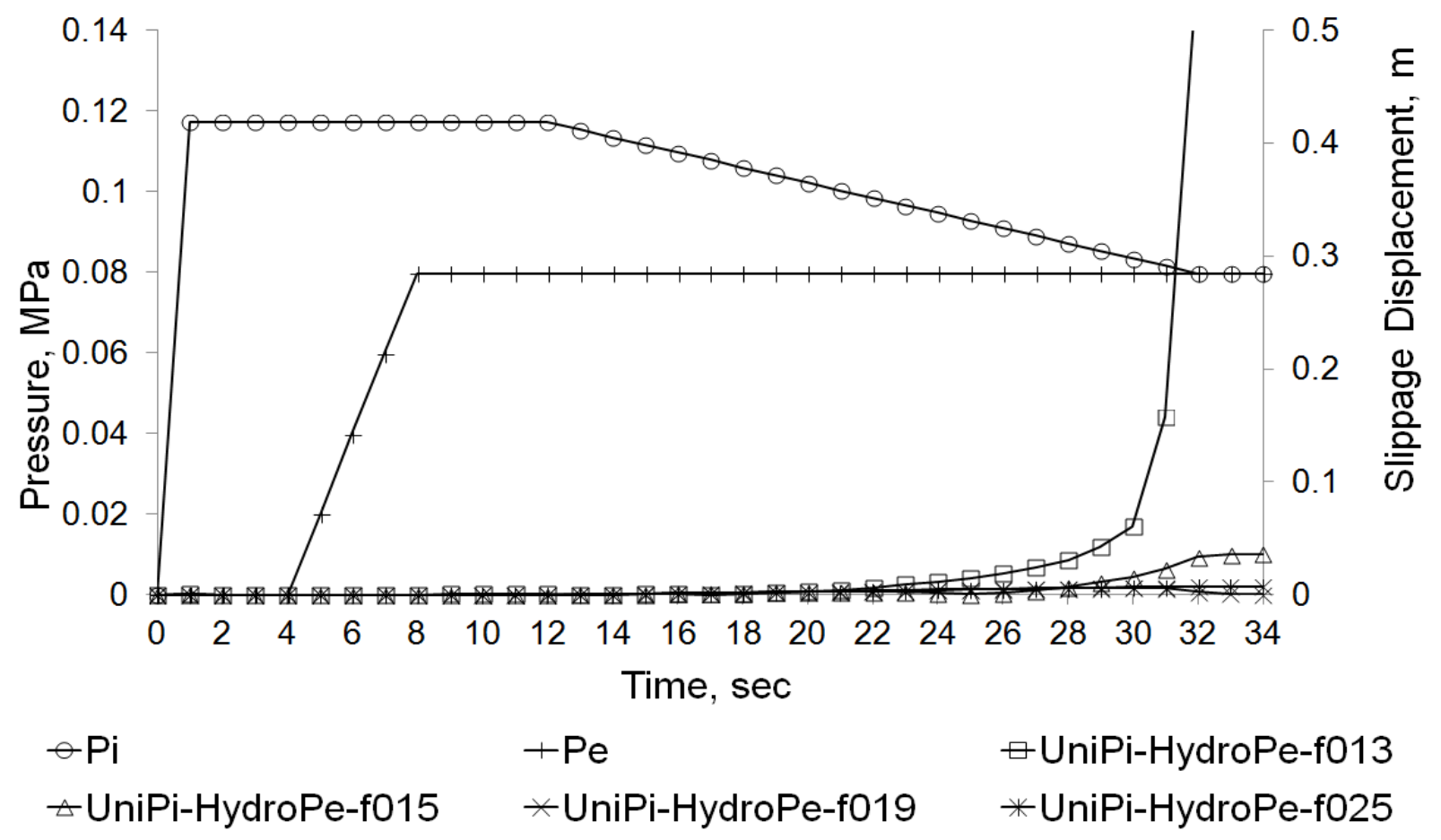

Figure 8.13: Slippage results for Group 6.

Table 8.5: Influence of Friction Coefficient on Holding Resistance Ratio.

\begin{tabular}{|c|c|c|c|}
\hline Group & Pressurization Event & Friction Coefficient, $\mu$ & $\mathrm{Pe} / \mathrm{Pi}$ ratio reached at slippage \\
\hline \multirow{4}{*}{6} & \multirow{4}{*}{$\begin{array}{l}\text { Assuming } \\
\text { Depressurization of } \\
\text { the Plug }\end{array}$} & 0.13 & 0.96 \\
\hline & & 0.15 & 0.98 \\
\hline & & 0.19 & 1.00 \\
\hline & & 0.25 & 1.00 \\
\hline
\end{tabular}




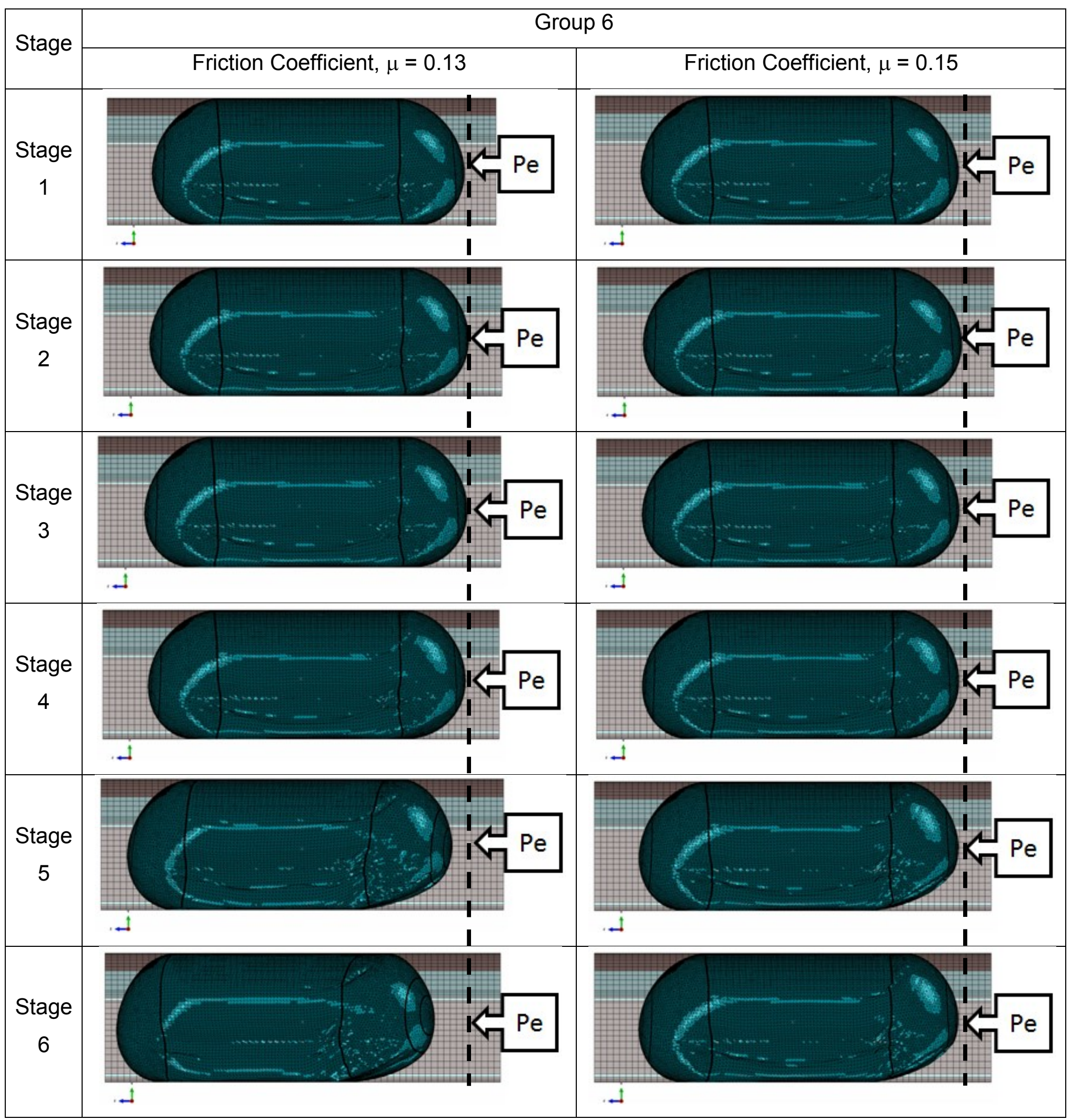

Figure 8.14: Sequence of slippage for friction coefficients of 0.13 and 0.15

(Deformation scale factor $=1$ ). 


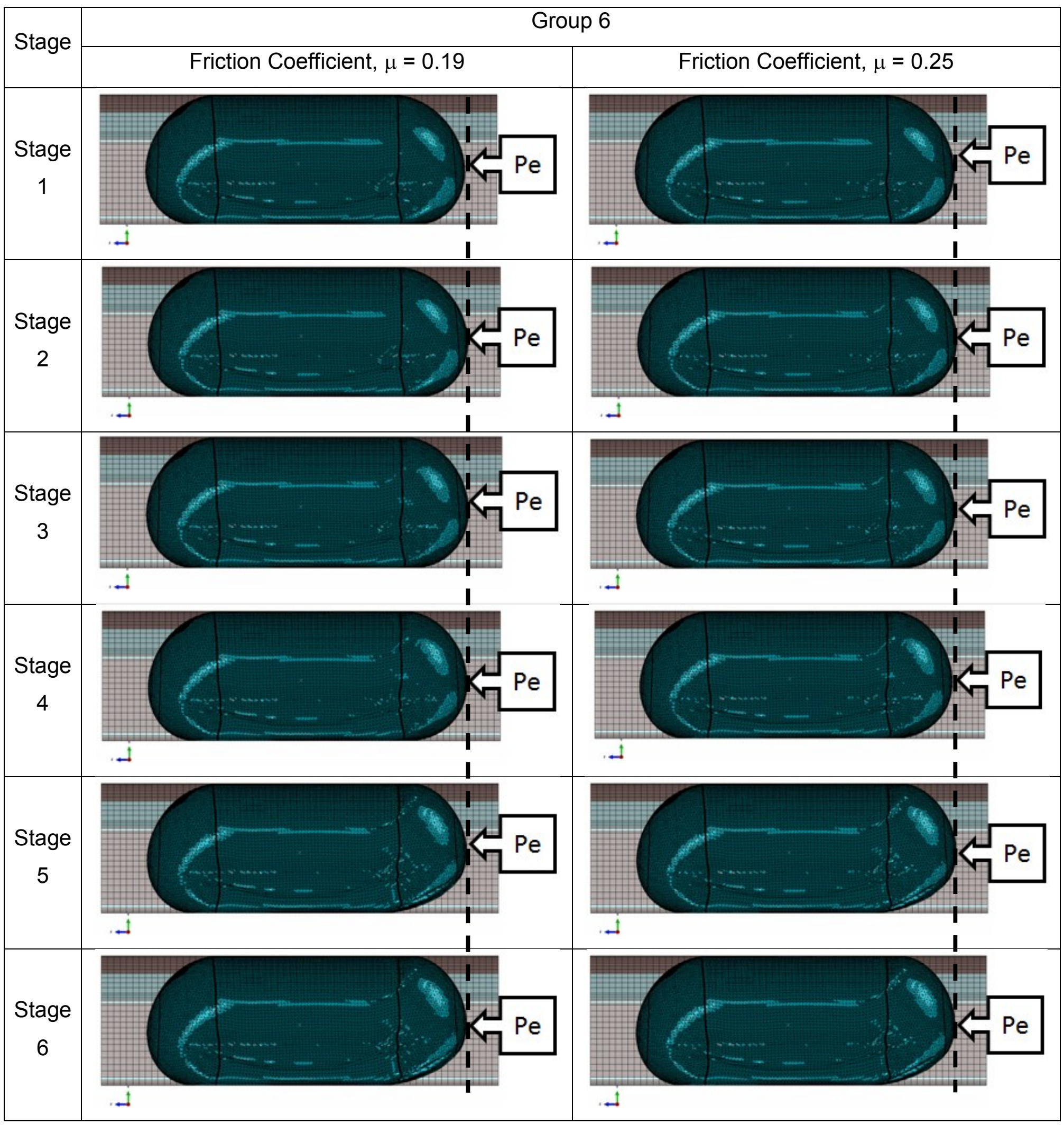

Figure 8.15: Sequence of slippage for friction coefficients of 0.19 and 0.25 .

(Deformation scale factor $=1$ ). 
Figure 8.16 shows the slippage results of Group 7 (UniPi-UniPe). In this group, the internal surface of plug has uniform pressure with fluid cavity (described in Chapter 2 , in which the fluid has mass and the characteristics of air) and the external uniform pressure is acting on the surface of the plug rear end-cap. Only the model with friction coefficient of 0.19 was analyzed and the results show that the plug slipped less than 0.1 $\mathrm{m}$ around the $31^{\text {st }}$ second before reaching a new equilibrium position for the remaining part of the simulation. The holding resistance for this case was 0.977 .

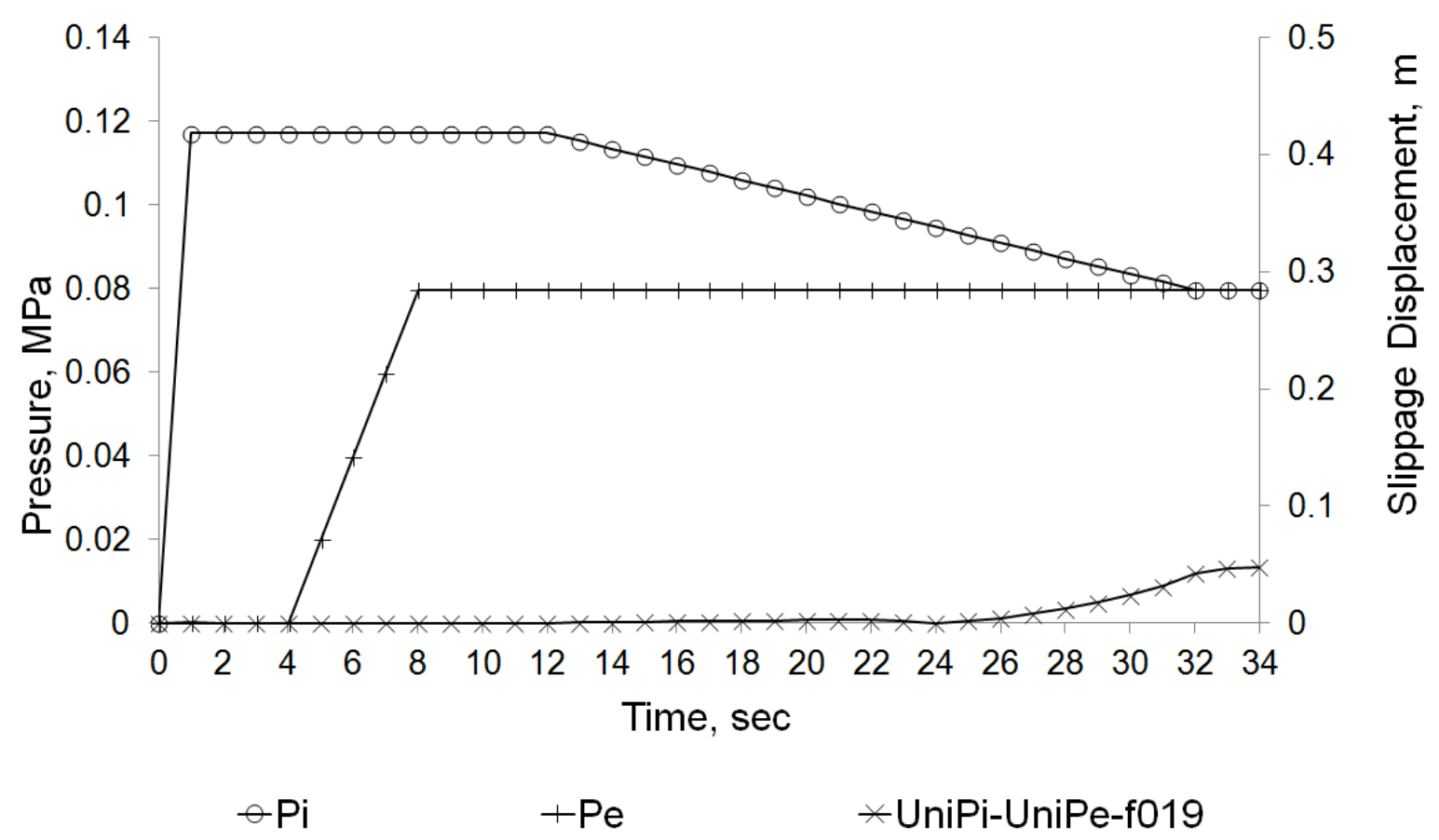

Figure 8.16: Slippage result for Group 7.

Lastly, Figure 8.17 shows the slippage results of Group 8 (HydroPi-UniPe). In this group, the internal surface of the plug has a hydrostatic pressure with fluid cavity (described in Chapter 2) and an external uniform pressure on the surface of the plug rear end-cap. This model was analyzed only with a friction coefficient of 0.19 and slipped twice, first of the $7^{\text {th }}$ second and then at the $17^{\text {th }}$ second the holding for this last case was resistance resulted approximately 0.73 . 


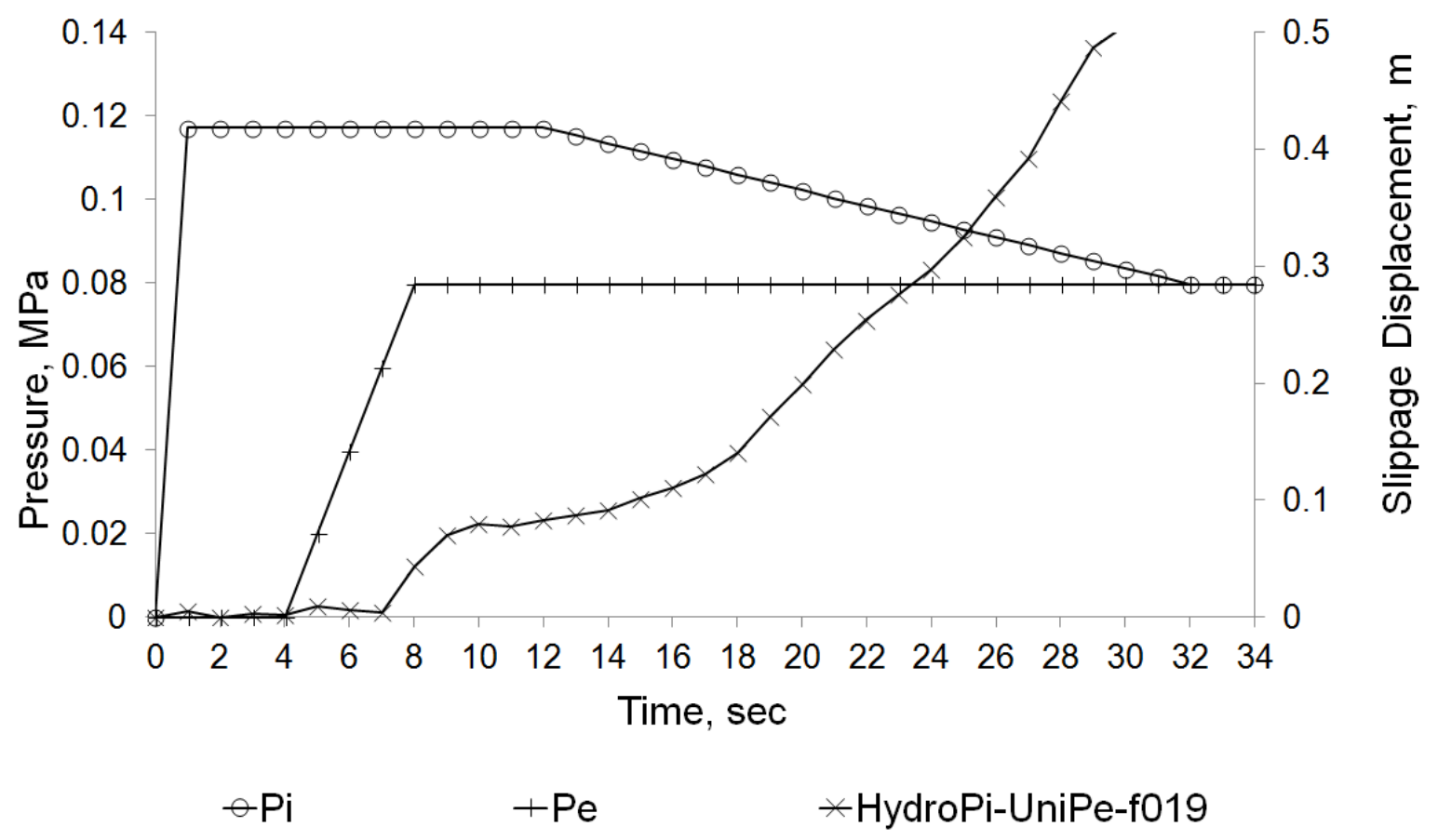

Figure 8.17: Slippage result for Group 8.

Table 8.6 shows a summary of the holding resistance results for Groups 5 to 8 (the cases that assumed depressurization of the plug). Similarly to Groups 1 and 2, Groups 5 and 6 are the other two groups that represent feasible testing scenarios and possible field scenarios. Group 6 shows higher holding resistance for friction coefficients of 0.13 and 0.15 (25\% and $17 \%$, respectively). Group 6 shows a higher holding resistance than Group 5 for a friction coefficient of $0.19(4 \%)$. Both groups displayed the maximum holding resistance of 1.0 for a friction coefficient of 0.25 . Group 8 showed the lowest holding resistance of all cases which indicated that the plug slipped as soon as the external pressure reached its maximum value applied on the surface of the plug rear end-cap as seen in Figure 8.19. Group 8 shows the model with friction coefficient of 0.19 obtained the lowest holding resistance of 0.73 , though more runs would be necessary to complete the modeling plan for other friction coefficients. Overall, holding resistance results corresponding to Groups 5 and 6 showed that a uniform pressure applied in the interior of the plug can hold the plug slightly better than a hydrostatic pressure. 
Table 8.6: Summary results of holding resistance of Groups 5 to 8.

\begin{tabular}{|c|c|c|c|c|}
\hline \multirow{2}{*}{$\begin{array}{c}\text { Friction } \\
\text { Coefficient, } \mu\end{array}$} & \multicolumn{4}{|c|}{ Holding resistance, Pe/Pi } \\
\cline { 2 - 5 } & $\begin{array}{c}\text { HydroPi- } \\
\text { HydroPe }\end{array}$ & $\begin{array}{c}\text { UniPi- } \\
\text { HydroPe }\end{array}$ & $\begin{array}{c}\text { HydroPi- } \\
\text { UniPe }\end{array}$ & $\begin{array}{c}\text { UniPi- } \\
\text { UniPe }\end{array}$ \\
\cline { 2 - 5 } & Group 5 & Group 6 & Group 7 & Group 8 \\
\hline 0.13 & 0.77 & 0.96 & - & - \\
\hline 0.15 & 0.84 & 0.98 & - & - \\
\hline 0.19 & 0.96 & 1.00 & 0.98 & 0.73 \\
\hline 0.25 & 1.00 & 1.00 & - & - \\
\hline
\end{tabular}

\subsection{Summary}

The evaluation of the axial stability of a model of a full-scale inflatable deployed inside a tunnel section was presented in this chapter. Models of slippage for different pressurization sequences and loading distributions were evaluated for several friction coefficients.

The results for pressurization sequences expected under normal operating conditions have shown that a friction coefficient of at least 0.15 is the minimum required by the system to remain stable and to withstand the required operating pressures.

A friction coefficient of 0.19 seems to be the threshold of the system to withstand slippage in an event of gradual depressurization of the plug.

Loading scenarios of denominated HydroPi-HydroPe and UniPi-HydroPe are feasible testing scenarios and possible field scenarios. Loading scenario of HydroPiHydroPe can be considered as a better scenario to examine the minimum requirement of system friction since the model with friction coefficient of 0.13 predicted the slippage occurring before depressurization. Loading scenario UniPi-HydroPe seems to provide better holding resistance ratios in an event of gradual depressurization of the plug. 


\section{Conclusions and Recommendations}

\subsection{Introduction}

This study consisted in the development of Finite Element (FE) models of inflatable structures at different scales used for protection of tunnels in an event of flooding. The modeling strategy was developed in two scales: first, a FE model of a reduced-scale inflatable, specifically one-fourth of the full dimensions, and; second, a FE model of a full-scale inflatable. In this way, several of the key components necessary to create a reasonably accurate model were initially developed and implemented in the model of the reduced-scale inflatable and later translated to the model of the full-scale inflatable. Two of the key properties evaluated in the model of the reduced-scale were the equivalent membrane mechanical properties and the system friction coefficient. The simulation results were able to replicate experimental results in terms of constrained elongation and slippage resistance. Then, the equivalent material properties and system friction coefficient were implemented in the model of the full-scale inflatable to simulate the deployment behavior, verify the local conformity, analyze the slippage characteristics, and the results were compared to available experimental results.

This chapter presents the main conclusions obtained from the simulation results obtained following the strategy outlined above. Possible future work is also recommended to expand the scope of this study.

\subsection{Conclusions for Reduced-Scale Prototype}

The modeling of a reduced-scale inflatable was the starting point for the development of this research. The FE models at this scale were used to determine and calibrate various parameters later used on the FE models of the full- scale inflatable.

The following sections summarize main results and conclusions of the simulation results including mesh convergence study, simplified deployment, stress evaluation, elongation evaluation, and slippage evaluation. 


\subsubsection{FE Model Creation}

The structural membrane of the inflatable was created using a single equivalent layer of membrane elements representative, in terms of mass and thickness, of the actual tri-layer system used for manufacturing the actual prototypes. The rope sections were assumed to have same material properties as the equivalent single layer as well as equivalent cross sectional area as the real ropes. Material properties such as membrane shear strength, friction coefficient between the inflatable and the tunnel, and self-friction of the woven webbing were obtained experimentally [59,60]. The tunnel and the inflation port fittings on the inflatable are assumed to be rigid bodies.

\subsubsection{Mesh Convergence Study}

Convergence studies were performed to determine the minimum number of elements necessary to converge to control membrane stress values obtained from analytical solutions within an acceptable level of error and reasonable computational cost.

The results of convergence studies showed that a FE model of the reduced-scale inflatable needs to be meshed with at least 10000 elements to converge to the theoretical values of stresses corresponding to an unconstrained pressurization condition with an error of less than $2 \%$.

\subsubsection{Simplified Deployment}

In order to place the deflated inflatable into a circular tunnel with minimal computational cost, a simplified approach for placement and deployment was created. This approach cannot be considered totally representative of actual deployment of a reduced-scale inflatable and served the purpose of placing the inflatable inside the tunnel. However, this simplified approach served as baseline for the development of some of the procedures implemented for placement of the model of the full-scale inflatable into a more realistic tunnel section. 


\subsubsection{Stress Evaluation}

Membrane stresses evaluation under unconstrained pressurization condition was performed to verify analytical values of hoop and longitudinal stresses. The results show that the model can approximate the theoretical values with a maximum error of $3.24 \%$.

On the other hand, the stress evaluation under constrained pressurization condition resulted $18 \%$ higher than the theoretical values in the hemispherical end-caps and, nearly $300 \%$ lower in cylindrical portion of the inflatable. The drastic reduction of the stresses in the cylindrical portion of the plug is attributed to the confining effect of the cylinder representative of a tunnel or pipe section that the inflatable is intended to seal.

\subsubsection{Elongation Evaluation}

A series of elongation tests in the reduced-scale prototype were modeled under constrained conditions to calibrate the equivalent material properties of the actual trilayer of fabric used to construct the inflatable plug. Material \#4 with a maximum tensile stress of $258.5534 \mathrm{MPa}(37500 \mathrm{psi})$ and a maximum tensile strain of 0.1 produced an elongation plot with the smallest error with respect to the linear trend line obtained from experimental results.

\subsubsection{Slippage Evaluation}

The purpose of performing the slippage simulations using the model of the reduced-scale inflatable was to determine the influence of the friction coefficient between inflatable and tunnel wall, on the longitudinal axial stability of the plug.

Twelve simulation cases were analyzed including four sets of loading scenarios (test IDs of $55 / 40,40 / 30,30 / 20$, and 20/10) with three different friction coefficients ( $\mu=0.15, \mu=0.19$, and $\mu=0.25$ ). In all these cases, the models simulated induced slippage by depressurization of the inflatable. The simulation results obtained for a system friction coefficient of 0.19 were comparable to results obtained experimentally 
[59]. The model with friction coefficient of 0.19 predicted an average holding resistance of 0.8283 which was only $3.5 \%$ higher than the average value obtained from experimental tests [59].

Moreover, an additional case was analyzed to verify the longitudinal axial stability when the model of the reduced-scale prototype was subjected to design pressures $(\mathrm{Pi}=0.4688 \mathrm{MPa}$ and $\mathrm{Pe}=0.3309 \mathrm{MPa})$ with four different friction coefficients $(0.15,0.19$, 0.21 , and 0.25$)$. The case for $(\mu=0.19)$ was confirmed experimentally [59] and taken as basis for comparison with the FE models developed for the full-scale prototype. The simulation results from other friction coefficients $(0.15,0.21$, and 0.25$)$ could not be validated due to unavailability of experimental results. However, this set of results provided an estimation of the magnitude of the friction coefficients to either induce or avoid slippage of the inflatable.

\subsection{Conclusions for Full Scale Prototype}

The following sections summarize the conclusions obtained from the simulation results of the full-scale inflatable including: initial preparation of the FE models, folding configuration, placement and relaxation, deployment, and evaluation of axial slippage.

\subsubsection{Initial Preparation}

The different components of the FE model including the full-scale inflatable, base plane, rotational plates, and tunnel section were initially created in preparation for the different stages of the simulation. Each of the components served for different purposes in each particular stage such as folding, placement, relaxation, and deployment.

The results of stress evaluation under unconstrained pressurization showed that the average hoop and longitudinal stresses at cylindrical region have errors of $2.69 \%$ and $2.91 \%$, respectively, when compared to theoretical values. The results of stress evaluation also showed an average error of $1.72 \%$ for hoop and longitudinal stresses at front and read hemispherical end caps. 
The convergence study suggested that a mesh with at least 20,000 elements (nearly 30,000 DOF) can predict the analytical stresses with an error of $2.69 \%$ with a reasonable computational cost.

\subsubsection{Folding Configuration}

Folding was the process that transformed a flattened inflatable structure into certain shape for situating it in a particular place or position within the tunnel section. A novel folding methodology using boundary conditions to control the nodes and elements initially designed to define the folding lines was proposed to be the folding. This methodology included procedures to add connector elements to the partially folded model between the folding steps, and to control the rigid plates used for folding of the inflatable and reproducing the actual folding process.

The modeling of the folding process developed in this study replicated an actual hand folding implemented experimentally. It essentially consisted on translating nodes and rigid body planes. The folding sequence included five steps to achieve the target shape after flattening. The penetration and intersection of elements needed to be verified to prevent element distortion happening during the deployment simulation.

\subsubsection{Placement and Relaxation}

The placement process consisted of a combination of rotations and translations done to position the folded inflatable within a determined storage area in the tunnel. The relaxation process helped to restore the elements back to initial coordinates as well as to prevent the elements from distortion.

The relaxation process was performed through associated invariants by developing the constitutive equations and the specific local in-plane membrane kinematics [118]. The relaxation process restored $96 \%$ of the total membrane area to its initial configuration with less than $0.5 \%$ of difference percentile between kinetic energy 
and internal energy. Therefore, the relaxation must be performed again at the stage of deployment in order to complete the restoration.

A simple evaluation of relaxation applied to a folded unconstrained folded plug was performed. This evaluation compared the volumes of the models with and with relaxation. The evaluation result showed that the model without relaxation predicted a volume approximately $18 \%$ smaller than the actual expected volume and remained with severe distortions in the shape after completion of the inflation. While the model that included relaxation was able to restitute all the original volume with no distortions in the surface of the membrane. This simple evaluation provided valuable information on the importance of including relaxation in the FE models in order to obtain the corrected volumes minimizing the formation of unrealistic distortions in further models.

\subsubsection{Deployment}

The inflation algorithm of Uniform Pressure Method (UPM) was chosen due to its relative low computational cost and its simplicity. The implementation of multi-chamber and fluid exchange approaches extended the capability of UPM to reproduce the actual deployment behavior. A scale factor of 386 needed to be assigned to the material properties of the connector elements due to effect of mass scaling in order to replicate the actual passive restraints representative of ties installed during the folding process. According to the results of deployment simulation, the inclusion of passive restraints demonstrated to be an effective solution to increase the level of local conformity. The simulations were also able to predict the position of wrinkles and bridging at similar positions as observed in the actual deployments. The percentage of improvement in terms of effective contact area between the plug and tunnel for the cases with and without connector elements is approximately $20 \%$. However, the FE deployment model has successfully simulated the actual deployment behavior through Uniform Pressure Method and created a model baseline by evaluating experimental data.

Simulations results showed that the presence and location of wrinkles is associated to the presence, or not, of the passive restraints used to control the release 
of membrane material during the deployment. Deployment \#1 and \#2 displayed wrinkles accumulated at the bottom corner of the tunnel due to the membrane material did not drive up the side wall of the tunnel. On the other hand, in Deployments \#3 and \#4 showed less wrinkling on the tunnel lateral wall because the passive restraints carried the membrane material up to the side wall on the wall after the ties broke during the deployment. The implementation of passive restraints contributed significantly to improve the local conformity of the plug to critical places such as corners, transitions and intricate elements located inside the tunnel section.

\subsubsection{Evaluation of Axial Slippage}

The longitudinal axial slippage resistance was evaluated under various load combinations and friction coefficients. The best conformity case among the four deployment cases (Deployment \#4) was selected to perform the axial slippage simulations.

The induced axial slippage tests were modeled for two different conditions: a) normal operating condition at the design pressures and b) assuming depressurization of the plug. Each condition was analyzed under four different loading scenarios with various friction coefficients. The simulation result of $\square=0.19$ under normal operating condition at the design pressures showed no slippage with the severest loading scenario of HydroPi-HydroPe (Hydrostatic pressure distributions for both $\mathrm{Pe}$ and $\mathrm{Pi}$ ) and it was confirmed experimentally [29]. For the simulation under assuming depressurization of the plug, the model with $\mu=0.19$ under loading scenario of HydroPiHydroPe slipped at 30th second and obtained the holding resistance ratio of 0.91 .

The results of slippage simulations provided an estimation of the system friction coefficient that would be necessary to install maintain in actual applications. At last, the finite element models were able to replicate successfully some of the available experimental results obtained with prototypes at full-scale. The simulation models proved also that they can be used as predicting tools of other testing configurations 
before the execution of the actual tests and for optimization and improvement of the design.

\subsection{Novel Contributions of Study}

The novel contributions made by this study for the development of FE models of confined inflatable plugs include:

- A hybrid folding methodology consisting of: using boundary conditions to control the nodes and elements that are part of the folding lines; adding new connector's elements between the folding steps, and; controlling the rigid plates to fold the full-scale inflatable according to an actual folding process.

- Implementation of multi-chambers in the modeling that can be translated to actual applications by manufacturing the multi-chambers within an inflatable structure. The opening and closing of the chambers in the inflatable structure can be controlled and deployed individually based on the particular conditions of the deployment site.

- $\quad$ Through slippage simulation of full-scale experiments, the simulation results demonstrated the advantages of using FE modeling to predict or measure physical quantities that cannot be obtained directly or immediately from experimental results.

\subsection{Recommendations}

\subsubsection{Recommendations for Simulations of Reduced-scale Prototype}

For FE model of reduced-scale confined inflatables, several suggestions are proposed for future work in order to get more information or to improve the current model.

I. Recommendations for model improvement:

a. Increase the number of element at the cylindrical portion of the inflatable for the model for better definition of conformity and wrinkles.

b. Create the rigid plates to perform the folding process on the inflatable.

c. Implement placement and relaxation process on the model.

d. Implement UPM technique to deploy the inflatable. 
II. Suggestions for future evaluations:

a. Replicate and verify the experimental data from the other set of loading scenario on the slippage test in the Ref [59].

b. Use the verified simulation data to produce models with different contact lengths of the plug and tunnel.

\subsubsection{Recommendations for Simulations of Full-Scale Prototype}

For FE model of full scale confined inflatables, several ideas are proposed to improve the current model in order to improve the predicting capabilities:

I. Recommendations for model improvement:

a. Increase the number of element at the cylindrical portion of the inflatable for the model for better definition of conformity and wrinkles.

b. Refine the folding process or propose alternative folding sequences that can potentially improve the local conformity and eliminate wrinkles.

c. The deployment behavior of current model can be improved by redesigning the longitudinal chambers and by creating more chamber walls on longitudinal direction (yz-plane). The discrete phase of fluid flow can be performed more smoothly.

II. Suggestions for future works:

a. Use current folded plug model and deploy in other tunnel profile.

b. Create another model with different folding configuration and deploy in the current tunnel profile to evaluate the performance of the deployment.

c. Implement the Coupled Euler-Lagrangian (CEL) or the Smoothed Particles Hydrodynamic (SPH) to predict the deployment behavior with current folding configuration and compare the results with available experimental data. 


\section{References}

1. Lana di Terzi F. (1670). Prodromo Overo saggio di alcune inventioni nuove premesso all'Arte maestra, Brescia: Rizzardi.

2. Davis T. (2009). Lighter Than Air, The Johns Hopkins University Press, 2.

3. Clement J., Warren A. W., \& Davis J. (1818). Aeronautics, Rest Fenner, Paternoster Row. Retrieved from http://www.loc.gov/pictures/item/2002722696

4. Thomson, R. W. (1847). U.S. Patent No. 5104. London: U.K.

5. Topham, S. (2002). Blow Up. Prestel - Verlag, Munich.

6. Shaeffer, R. E. (2006, September/October). The Last Night. Fabric Architecture, 58-61.

7. Dessauce, M., Aubert, J., Jungmann, J. \& Stinco, A. (1999). The inflatable moment: pneumatics and protest in '68. New York: Princeton Architectural Press.

8. National Aeronautics and Space Administration. (n.d.). SP-4308 SPACEFLIGHT REVOLUTION: The Odyssey of Project Echo. Retrieved January 10, 2012, from http://history.nasa.gov/SP-4308/ch6.htm

9. Shah, A. (2010, January-March). Frei Otto-Pioneer in light weight tensile and membrane structures. Structural Engineering Digest. Retrieved from http://sedigest.in/review/frei-otto-pioneer-light-weight-tensile-and-membranestructures

10. National Aeronautics and Space Administration. (n.d.). Mars Pathfinder Airbags. Retrieved January 10, 2012, from http://mars.jpl.nasa.gov/MPF/mpf/mpfairbags.html

11. Keith, H. (2007, February 23). Camping on the Moon Will Be One Far Out Experience. Retrieved from http://www.nasa.gov/exploration/home/inflatablelunar-hab.html

12. American Ergonomics Corporation. (2006). Counter Balance Motion Seat for Automotive Safety. Retrieved from http://americanergonomics.com/?page=auto

13. Composites World. (2009, January 01). The markets: Civil infrastructure (2011). Retrieved from http://www.compositesworld.com/articles/the-markets-civilinfrastructure-2010 
14. Martinez, X., Davalos, J., Barbero, E., Sosa, E., Huebsch, W., Means, K., Banta, L., \& Thompson, G. (2012, May 21-24). Inflatable Plug for Threat Mitigation in Transportation Tunne/s. Paper presented at Society for the Advancement of Material and Process Engineering 2012 Spring Conference, Baltimore, MD.

15. Barbero, E.J., Sosa, E.M., Martinez, X., \& Gutierrez, J.M. (2013, June). Reliability Design Methodology for Confined High Pressure Inflatable Structures.

Engineering Structures, 51, 1-9. http://dx.doi.org/ 10.1016/j.engstruct.2013.01.011

16. World Socialist Web Site. (2003, February 24). Inadequate Safety Planning Produces South Korean Subway Disaster. Retrieved from http://www.wsws.org/articles/2003/feb2003/daeg-f24

17. Arnold, R.L. (n,d). Special Report: Underground Flood Hits Chicago's Loop, Shutting Down Businesses for Weeks. Disaster Recovery Journal. Retrieved from http://www.drj.com/special/chicago.html

18. Landers, P., Zaun, T., \& Fialka. (2001, September 28). In 1995 Tokyo Gas Attack, Lessons for the U.S.. The Wall Street Journal, A12.

19. CBS News. (2007, August 31). Deadly Blast Hits Moscow. Retrieved from http://www.cbsnews.com/stories/2004/08/31/world/main639738.shtml

20. Flegenheimer, M. (2012, October 31). Flooded Tunnels May Keep City ‘s Subway Netwrok Closed for Several Days. The New York Times, A17.

21. Tony, P. (n.d). 2nd Manapouri Tailrace Tunnel 1998. Tunnel \& Mining New Zealand. Retrieved August 16, 2012, from http://demo.idesign.co.nz/tunnel/index.php?option=com_content\&task=view\&id=2 $1 \&$ ltemid $=48$

22. Bavani, M. (2007, April 16). Opening of SMART tunnel to reduce flood risks and traffic jams, The Star Online. Retrieved from http://thestar.com.my/news/story.asp?file=/2007/4/16/central/17446085\&sec=cent ral

23. Halcrow. (n.d). Our history - Deep Level Air Raid Shelters, London 1942. Retrieved August 16, 2012, from http://www.halcrow.com/Who-weare/History/Deep-Level-Shelters-London---1942/ 
24. Petersen Products Co. (n.d). Grout Bags, Inflatable Tunnel Seals. Retrieved August 16, 2012, from

http://www.petersenproducts.com/groutbagstunnelseals.aspx

25. Petersen Products Co. (n.d). Custom Bladders and Inflatable Products. Retrieved August 16, 2012, from http://www.petersenproducts.com/custom.aspx

26. Lindstrand Technologies. (n.d.). Inflatable Tunnel Plugs. Retrieved August 16, 2012, from http://www.lindstrandtech.com/innovation_centre.html

27. Lindstrand Technologies. (n.d.). Successful inflatable tunnel plug tests in the Netherlands. Retrieved August 16, 2012, from http://www.lindstrandtech.com/news4.html

28. WVU College of Engineering and Mineral Resources. (2009). Tunnel Safety: Focus on Research Effort. WVU College of Engineering and Mineral Resources EngineeringWV Magazine, 5(1), 4. Retrieved from http://www.cemr.wvu.edu/news/magazine/EngineeringWV_Spring09.pdf

29. Dillon, M.C. (2012). Resilient Tunnel Plug Continues to Impress Research Partners. WVU College of Engineering and Mineral Resources EngineeringWV Magazine, 8(1), 7. Retrieved from http://www.cemr.wvu.edu/news/magazine/EngineeringWV_Spring12.pdf

30. Bower, A. F. (2009, October 5). Applied Mechanics of Solid. Boca Raton, FL. CRC Press.

31. Green, A. E., \& Adkins, J. E. (1954). Large Elastic Deformations, Clarendon Press.

32. Turner, M. J., Clough, R. W., \& Topp, L.J. (1956). Stiffness and Deflection Analysis of Complex Structures. Journal of the Aeronautical Sciences, 23, 805823.

33. Oden, J.T. (1966). Analysis of Large Deformations of Elastic Membranes by the Finite Element Method. Proceedings from IASS: Congress on ... Large Span Shells. Leningrad, USSR.

34. Oden, J.T. (1967, June). Numerical Formulation of Nonlinear Elasticity Problems. Journal of Engineering Mechanics Division, ASCE.

35. Oden, J.T., \& Kubitza, W.K. (1967). Proceedings from IASS: The First International Colloqium on Pneumatic Structures. Stuttgart, Germany. 
36. Oden, J.T., \& Sato, T. (1967). Finite Strains and Displacements of Elastic Membranes by the Finite Element Method. International Journal of Solids and Structures, 3, 471-488.

37. Flores, F.G., \& Oñate, E. (2004). Improvements in the membrane behavior of the three node rotation-free BST shell triangle using an assumed strain approach. Computer Methods in Applied Mechanics and Engineering, 194, 907-932.

38. Oñate, E., \& Flores, F. G. (2005). Advances in the formulation of the rotation-free basic shell triangle. Computer Methods in Applied Mechanics and Engineering, 194, 2406-2443.

39. Taylor, R. L., Oñate, E., \& Ubach, P. (2005). Finite Element Analysis of Membrane Structures. Computational Methods in Applied Sciences, 3, 47-68.

40. Oñate, E., Flores, F.G., \& Marcipar, J. (2008). Membrane Structures Formed by Low Pressure Inflatable Tubes. New Analysis Methods and Recent Constructions. Computational Methods in Applied Sciences, 8, 163-196.

41. Rossi, R., Lazzari, M., Vitaliani, R., \& Oñate, E. (2005). Simulation of Light-weight Membrane Structures by Wrinkling Model. International Journal Numerical Methods Eng, 62, 2127-2153.

42. Dassault Systèmes Simulia Corp. (2011). Abaqus Theory Manual, Abaqus 6.11 Documentation Collection.

43. Dassault Systèmes Simulia Corp. (2011). Abaqus Analysis User's Manual, Abaqus 6.11 Documentation Collection.

44. Hugoniot, H. (1887). Mémoire sur la propagation des mouvements dans les corps et spécialement dans les gaz parfaits (première partie) [Memoir on the propagation of movements in bodies, especially perfect gases (first part)]". Journal de l'École Polytechnique (in French), 57, 3-97.

45. Hugoniot, H. (1889). "Mémoire sur la propagation des mouvements dans les corps et spécialement dans les gaz parfaits (deuxième partie)" [Memoir on the propagation of movements in bodies, especially perfect gases (second part)], Journal de l'École Polytechnique (in French), 58, 1-125.

46. Mie, G. (1903) "Zur kinetischen Theorie der einatomigen Körper"[ The kinetic theory of monatomic body], Annalen der Physik (in German), 316, 657-697. 
47. Grüneisen, E. (1912). Theorie des festen Zustandes einatomiger Elemente [Theory of the solid state of monoatomic elements], Annalen der Physik (in German), 344, 257-306.

48. Nakayama, T., \& Washizu, K. (1980). Nonlinear Analysis of Liquid Motion in a Container Subjected to Forced Pitching Oscillation. International Journal for Numerical Methods in Engineering, 15, 1207-1220.

49. Dassault Systèmes Simulia Corp. (2011). Abaqus Example Problem Manual, Abaqus 6.11 Documentation Collection.

50. Dassault Systèmes Simulia Corp. (2011). Abaqus Benchmarks Manual, Abaqus 6.11 Documentation Collection.

51. Cannmo, P. \& Snygg, H. (2001). Proceedings from 2001 IMAC XIX - 19th International Modal Analysis Conference. Orlando, FL.

52. Higuchi, M., Tanaka, T., \& Endo, S. (1976). Study on hull vibration-induced tank liquid sloshing in LPG tankers, Nippon Kokan Tech. Rep. (in Japanese), 72, 111122.

53. Jetteur, P., \& Bruyneel, M. (2008). Advanced Capabilities for the Simulation of Membrane and Inflatable Space Structures Using SAMCEF. Textile Composites and Inflatable Structures II, 211-231, Springer.

54. Rumpel T., Schweizerhof K., HaBler M. (2005). Efficient Finite Element Modeling and Simulation of Gas and Fluid Supported Membrane and Shell Structures. Textile Composites and Inflatable Structures, 153-172, Springer.

55. Rumpel, T., \& Schweizerhof, K. (2003). Volume-dependent Pressure Loading and its Influence on the Stability of Structures. International Journal for Numerical Methods in Engineering, 56, 211-238.

56. Kuraray America Inc. (n.d.). Vectran ${ }^{\circledR}$ Liquid Crystal Polymer Fiber: Brochure \& Product Information. Retrieved January 10, 2012, from http://www.vectranfiber.com/BrochureProductlnformation.aspx

57. Cadogan D, Sandy C., Grahne M. (2002). Development and Evaluation of the Mars Pathfinder Inflatable Airbag Landing System. Acta Astronautica. 50(10), 633-640. Retrieved from http://www.sciencedirect.com/science/article/pii/S0094576501002156 
58. Barbero, E.J., Sosa, E. M. and Thompson, G.J. (2013). Testing of Full-Scale Confined Inflatable for the Protection of Tunnels. VI International Conference on Textile Composites and Inflatable Structures, Structural Membranes 2013. Munich, Germany.

59. Ghosh, S. (2012). Evaluation of Reduced-Scale Confined Inflatable Structure for Tunnel Safety. (Master's Thesis). Retrieved from West Virginia University Libraries. (http://www.libraries.wvu.edu)

60. Peil. K. L. (2012). Material Characterization and Analysis of Vectran Fabrics and Tensile testing for Inflatable Structure Applications. (Master's Thesis). Retrieved from West Virginia University Libraries. (http://www.libraries.wvu.edu)

61. Olovsson, L. \& Simonsson, K. (2006). Iterative Solution Technique in Selective Mass Scaling. Communications in Numerical Methods in Engineering, 22, 77-82.

62. Jung, D.W. (1998). Study of Dynamic Explicit Analysis in Sheet Metal Forming Process Using Faster Punch Velocity and Mass Scaling Scheme. Journal of Materials Engineering and Performance, 7, 479-490.

63. Pan, F., Zhu, J., Helminen, A. O., \& Vatanparast, R. (2006). Proceedings from $9^{\text {th }}$ International LS-DYNA Users Conference. Dearborn, Michigan: Livermore Software Technology Corporation.

64. ESI Group. (n.d.). Occupant Safety. Retrieved from http://virtualperformance.esigroup.com/applications-occupant-safety/

65. Oasys Ltd. (n.d.). Oasys PRIMER. Retrieved from http://www.oasyssoftware.com/dyna/en/software/primer.shtml

66. Livermore Software Technology Corporation. (2011). LS-PrePost. Retrieved from http://www.Istc.com/products/ls-prepost

67. Altair HyperWorks. (2012). Altair HyperCrash: Altair HyperCrash Capabilities. Retrieved from http://www.altairhyperworks.com/HWTemp3Product.aspx?product_id=31\&item_n ame=Capabilities

68. Forkin D. (2009, November 3). Airbag Folding with Radioss Pre- Simulation. Retrieved from 
http://www.altairhtc.com/europe/Presentations_2009/Session_02/ALTAIR_Airbag _Folding_ehtc_003b.pdf

69. Engineering Technology Associates, Inc. (2012). SAFETY. Retrieved from http://www.eta.com/vpg-modules/safety

70. TECOSIM. (2012). Occupant Safety. Retrieved from http://www.tecosim.com/services/structure-simulation/crash-simulation/occupantsafety.html? L=0

71. Gärtner, T., Erisson, M., \& Faltstorm, J. (1999, June). Advanced Technology for the Simulations of Folded Airbags. Paper presented at the $2^{\text {nd }}$ European LSDYNA Conference, Gothenburg, Sweden. Retrieved from http://www.carhs.de/en/company/publications/dyna_tor/dyna_tor.htm

72. Lee, J.K., Ha, W.P., Lee, J.H., Chae, D.B., \& Kim, J.H. (2009). Proceedings from 21st International Technical Conference on the Enhanced Safety of Vehicles Conference (ESV). Germany. Retrieved from http://wwwnrd.nhtsa.dot.gov/pdf/esv/esv21/09-0363.pdf

73. Rieger, D. (2003). Capability of simplified folded airbag models in gasflow simulations. European MADYMO users meeting, Brussels, Belgium

74. Engineering Technology Associates, Inc. (2012). Appendix C: VPG Airbag Folding User Manual. Retrieved from http://mirror.eta.com/Software/VPG/VPG31/VPG_3_Manual/Appendicies/Appendi x\%20C\%20Airbag\%20Folding\%20Manual.pdf

75. Tanavde, A., Khandelwal, H., Lasry, D., Ni, X. et al. (1995). Airbag Modeling Using Initial Metric Methodology. Journal of Passenger Cars, 104, 1576-1589.

76. Zhang, J., Ma, C., Bai, Y., \& Huang, S. (2005). Airbag Mapped Mesh AutoFlattening Method. Tsinghua Science and Technology, 10, 387-390.

77. Park, W. and Hong, S., (2005). A Study on the Modelling Technique for the Passenger Out-Of-Position Simulation. SAE Technical Paper 2005-01-1298, doi:10.4271/2005-01-1298.

78. Cromvik C. (2007). Numerical Folding of Airbags Based on Optimization and Origami. (Licentiate Thesis). Chalmers University of Technology and Göteborg University, Göteborg, Sweden. 
79. Chawla, A., Bhosale, P., \& Mukherjee, S. (2005). Modeling of Folding of Passenger Side Airbag Mesh. SAE Technical Paper 2005-26-059, doi:10.4271/2005-26-059.

80. Bosio, C., \& Mahangare, M. (2009). Rapid Airbag Folding Technique for Side Curtain Airbag. The $6^{\text {th }}$ Altair CAE Technology Conference 2009, 5, 1-10.

81. Liu, C. (2007). Dynamic Finite Element Modeling for the Conventional Spinning Process. Journal of the Chinese Institute of Engineers, 30, 911-916.

82. Wang J. T., Chen, T., Sleight, D.W., \& Tessler, A.(2004). Simulating Nonlinear Deformations of Solar Sail Membranes Using Explicit Time Integration. 45th AIAA/ASME/ASCE/AHS/ASC Structures, Structural Dynamics, and Materials Conference, AIAA-2004-1580, 741-755.

83. Underwood, P. (1983). Dynamic Relaxation. In T. Belytschko and T.J.R. Hughes, Computational Methods for Transient Analysis (pp. 245-265). New York, NY: Elsevier.

84. Otter, J.R.H., \& Day, A.S. (1960). Tidal Computations. The Engineer, 209, 177182.

85. Day, A.S. (1965). An Introduction to Dynamic Relaxation. The Engineer, 219, 218221.

86. Otter, J.R.H. (1965). Computations for Prestressed Concrete Reactor Pressure Vessels Using Dynamic Relaxation. Nucl. Struct. Engng, 1, 61-75.

87. Otter, J.R.H. (1966). Dynamic Relaxation. Proc. Inst. Civ. Engrs, 35, 633-656.

88. Otter, J.R.H., Cassel, E., \& Hobbs, R.E. (1966). Dynamic Relaxation. Proc. Inst. Civ. Engrs, 35, 723-750.

89. Han, S., \& Lee, K. (2003). A Study of the Stabilizing process of unstable structures by dynamic relaxation method. Computers and Structures, 81, 16771688.

90. Barnes, M.R. (1988). Form-finding and Analysis of Prestressed Nets and Membranes. Computers and Structures, 30, 685-695.

91. Barnes, M.R. (1999). Form Finding and Analysis of Tension Structures by Dynamic Relaxation. International Journal of Space Structures, 14, 89-104. 
92. Riches, C.G. \& Gosling, P.D. (1998). Proceedings from IASS International Congress '98: Lightweight Structures in Architecture, Engineering and Construction. Sidney, Australia.

93. Wood, R.D. (2002). A Simple Technique for Controlling element distortion in Dynamic Relaxation Form-finding of Tension Membranes. Computers and Structures, 80, 2115-2120.

94. Wu, T., \& Ting, E.C. (2008). Large Deflection Analysis of 3D Membranes Structures by a 4-node Quadrilateral Intrinsic Element. Thin-Walled Structures, 46, 261-275.

95. Rodriguez, J., Rio, G., Cadou, J.M., \& Troufflard, J. (2011). Numerical Study of Dynamic Relaxation with Kinetic Damping Applied to Inflatable Fabric Structures with Extensions for 3D solid Elements and Non-Linear Behavior. Thin-Walled Structures, 49, 1468-1474.

96. Welsh, A.K. (1967). Discussion on Dynamic Relaxation. Proc. Inst. Civ. Engrs. 37, 723-750.

97. Cassell, A.C. et al. (1968). Cylindrical Shell Analysis by Dynamic Relaxation. Proc. Inst. Civ. Engrs. 39, 75-84.

98. Rushton, K.R. (1968). Large Deflection of Variable-Thickness Plates. Int. J. Mech. Sci., 10, 723-735.

99. Wood, W.L. (1967). Comparison of Dynamic Relaxation with Three Other Iterative Methods, Engineer, 224, 683-687.

100. Cassell, A.C. (1970). Shells of Revolution Under Arbitrary Loading and the Use of Fictitious Densities in Dynamic Relaxation. Proc. Inst. Civ. Engrs., 45, 65-78.

101. Rushton, K.R. (1972). Buckling of Laterally Loaded Plates Having Initial Curvature. Int. J. Mech. Sci., 14, 667-680.

102. Alwar, R.S., \& Rao, N.R. (1973). Nonlinear Analysis of Orthotropic Skew Plates. AIAA J., 11, 495-498.

103. Alwar, R.S., and Rao, N.R. (1974). Large Elastic Deformations of Clamped Skewed Plates by Dynamic Relaxation. Computers and Structures, 4, 381-398. 
104. Frieze, P.A., Hobbs, R.E., \& Dowling, P.J. (1978). Application of Dynamic Relaxation to the Large Deflection Elasto-Plastic Analysis of Plates. Computers and Structures, 8, 301-310.

105. Pica, A., \& Hinton, E. (1980). Transient and Pseudo-Transient Analysis of Mindlin Plates. Int. J. for Num. Meth. in Engrg., 15, 189-208.

106. Key, S.W., Stone, C.M., \& Kreig, R.D. (1981). A Solution Strategy for the QuasiStatic, Large Deformation, Inelastic Response of Axisymmetric Solids. In W. Wunderlich et al., Nonlinear Finite Element Analysis in Structural Mechanics (pp. 585-620). New York, NY: Springer

107. Brew, J.S. \& Brotton, D.M. (1971). Nonlinear Structural Analysis by Dynamic Relaxation. Int. J. for Num. Meth. in Engrg. 3, 145-147

108. Wood, W.L. (1971). Note on Dynamic Relaxation. Int. J. for Num. Meth. in Engrg, 3, 145-147.

109. Bunce, J.W. (1972). A Note on the Estimation of Critical Damping in Dynamic Relaxation. Int. J. for Num. Meth. in Engrg. 4, 301-304.

110. Alwar. R.S., Rao, N.R., \& Rao, M.S. (1975). An Alternative Procedure in Dynamic Relaxation. Computers and Structures, 5, 271-274.

111. Cassell, A.C. \& Hobbs, R.E. (1976). Numerical Stability of Dynamic Relaxation Analysis of Non-Linear Structures. Int. J. for Num. Meth. in Engrg. 10, 1407-1410.

112. Papadrakakis, M. (1981). A Method for the Automated Evaluation of the Dynamic Relaxation Parameters. Computer Methods in Applied Mechanics and Engineerinf, $25,35-48$

113. Livermore Software Technology Corporation . (2007). LS-DYNA Keyword User's Manual.

114. Livermore Software Technology Corporation . (1999). LS-DYNA Theory Manual.

115. Dassault Systèmes Simulia Corp. (2011). Abaqus Keywords Reference Manual, Abaqus 6.11 Documentation Collection.

116. Zienkiewicz, O.C. (1977). The Finite Element Method. $3^{\text {rd }}$ Edition, McGraw-Hill.

117. Bathe, K. (1982). Finite Element procedures in engineering analysis. NJ: PrenticeHall 
118. Bonet, J., Wood, R.D., Mahaney, J., \& Heywood, P. (2000). Finite Element Analysis of Air Supported Membrane Structures. Computer Methods in Applied Mechanics and Engineering, 190, 579-595.

119. Hirth, A., Haufe, A., \& Olovsson, L. (2007). Airbag Simulation with LS-DYNA PastPresent-Future. Retrieved from http://www.dynamore.de/en/downloads/papers/07forum/keynote/airbag-simulation-with-Is-dyna-past-2013-present

120. Wang, J.T., \& Nefske, D. J. (1988). A New CAL3D Airbag Inflation Model. International Congress and Exposition, SAE 880654, Detroit.

121. Sinz, W. \& Hermann, S. (2008). The development of a 3D-Navier-Strokes code for the simulation of an airbag inflation. Simulation Modeling Practice and Theory, 16, 885-899. doi: 10.1016/j.simpat.2008.03.005

122. Sandia National Laboratories. (2002, May). Coupled Eulerian-Lagrangian Methods for Earth Penetrating Weapon Applications. Albuquerque, NM: Brown, K. H., Burns, S. P., \& Christon, M. A.

123. Birnbaum, N. K., Francis, N. J., \& Gerber, B. I. (n.d.). Coupled Techniques for the Simulation of Fluid-Structure and Impact Problems. Retrieved from http://hsrlab.gatech.edu/AUTODYN/papers/paper63.pdf

124. Quan, X., Birnbaum, N. K., Cowler, M.S., Gerber, B. I., Clegg R.A., \& Hayhurst, C. J. (2003). Numerical Simulation of Structural Deformation under Shock and Impact Loads using a Coupled Multi-solver Approach. Retrieved from http://hsrlab.gatech.edu/AUTODYN/papers/paper152.pdf

125. Noh, W. F. (1964). CEL: A Time-Depedent, Two-Space-Dimensional, Coupled Eulerian-Lagrangian Code. Methods in Computational Physics, 3, 117-179.

126. Benson, K. G. (1992). Computational Methods in Lagrangian and Eulerian Hydrocodes. Computer Methods in Applied Mechanics and Engineering, 99, 235394.

127. Belytschko, T., Flanagan, D.P., \& Kennedy, J.M. (1982). Finite Element Methods with User-Controlled Meshes for Fluid-Structure Interaction. Computer Methods in Applied Mechanics and Engineering, 33, 669-688. 
128. Donea, J., Giuliani, S., \& Halleux, J.P. (1982). An Arbitrary Lagrangian-Eulerian Finite Element Method for Transient Dynamics Fluid-Structure Interaction. Computer Methods in Applied Mechanics and Engineering, 33, 689-723.

129. Olovsson, L., (2000). On the Arbitrary Lagrangian-Eulerian Finite Element Method. (Doctoral Dissertation). Retrieved from http://www.solid.iei.liu.se/Publications/PhD_thesis/olovsson.html

130. Livermore Software Technology Corporation . (2005). LS-DYNA Theoretical Manual.

131. Gingold R. A., \& Monaghan J. J. (1977). Smoothed particle hydrodynamics: theory and application to non-spherical stars. Monthly Notices of the Royal Astronomical Society, 181, 375-389.

132. Wingate, C. A., \& Stellimhwerf, R. F. (1993). Smooth Particle Hydrodynamics: The SPHINX and SPHC Codes. Advances in Numerical Simulation Techniques for Penetration and Perforation of Solids, 171, 75-94.

133. Kuhnert, J. (1999). General Smoothed Particle Hydrodynamics. (Doctoral Dissertation). Retrieved from University Library Kaiserslautern.

134. Lian, W., Bhalsod, D., \& Olovsson, L. (2008). Benchmark Study of the AIRBAG_PARTICLE Method for Out-Of-Position Applications. $10^{\text {th }}$ International LS-DYNA Users Conference, Michigan.

135. National Institute of Standards and Technology. (2011). NIST Chemistry WebBook. Retrieved from http://webbook.nist.gov/chemistry/

136. Kyle, B. G. (1984). Chemical and Process Thermodynamics. Englewood Cliffs, NJ: Prentice-Hall.

137. Kobe, K. A. \& Lynn, R. E., Jr. (1953). Chemical Review, 52, 117-236.

138. American Society of Heating, Refrigerating, and Air-Conditioning Engineers, Inc. (1993). ASHRAE. Handbook of Fundamentals, 16.4 \& 36.1.

139. National Aeronautics and Space Administration. (n.d). Air Properties Definitions. Retrieved January 10, 2012, from https://www.grc.nasa.gov/WWWWright/airplane/airprop.html

140. Fokin, D., Dessarud, E., \& Ljungqvist, C. (2007). Simulation of Curtain Airbag with Arbitrary Eulerian-Lagrangian Method. $6^{\text {th }}$ LS-DYNA Forum, Frankenthal. 
141. Feng, B., \& Coleman, D. (2008). Gas Dynamic Simulation of Curtain Airbag Deployment through Interior Trims. $10^{\text {th }}$ International LS-DYNA Users Conference, Michigan.

142. Bird, R. B., Stewart, W. E. \& Lightfoot, E. N. (2002). Transport Phenomena. New York: Wiley.

143. Teng, H., Wang, J., \& Bhalsod, D. (2010). The Recent Progress and Potential Applications of Corpuscular Method in LS-DYNA®. In 11th International LSDYNA® Users Conference.

144. Zhang, H., Gopal, M., Saxena, R., and Avula, X. (2003). An Integrated Optimization System for Airbag Design and Modeling by Finite Element Analysis. SAE Technical Paper 2003-01-0506, doi:10.4271/2003-01-0506.

145. Lienard, S., \& Lefevre, Y. (2005). Modeling and Analysis of the Deployment of a Rolled Inflatable Beam Using MSC-DYTRAN, AIAA paper, AIAA-2005-1968.

146. Slade, R., Kiley, A., \& Toropov, V. (2007). Design optimisation and probabilistic assessment of a vented airbag landing system for the ExoMars space mission. 6th ASMO UK / ISSMO Conf. on Engineering Design Optimization. Process and Product Improvement. Oxford.

147. Salama, M., Kou, C.P., \& Lou, M. (2000). Simulation of Deployment Dynamics of Inflatable Structures. AIAA Journal, 38, 2277-2283. 


\section{Appendix A: Theoretical Calculation of Stress for Quarter Scale Prototype}

i. Spherical Cap Region:

The theoretical calculation for,

Hoop stress, $\quad S_{11}=\frac{P R}{2 t}$

Longitudinal stress, $\quad S_{22}=\frac{P R}{2 t}$

$$
\begin{aligned}
& =58 \mathrm{psi}(49 / 2 \mathrm{in}) /\left(2^{*} 0.307 \mathrm{in}\right) \\
& =2314.33 \mathrm{psi} \\
& =15.96 \mathrm{MPa}
\end{aligned}
$$

ii. Cylindrical Region:

The theoretical calculation for

$$
\text { Hoop stress, } \quad \begin{aligned}
S_{11} & =\frac{P R}{t} \\
& =58(49 / 2) /(0.307) \\
& =4628.66 \mathrm{psi} \\
& =31.91 \mathrm{MPa}
\end{aligned}
$$

The theoretical calculation for

$$
\text { Longitudinal stress, } \quad \begin{aligned}
S_{22} \quad & =\frac{P R}{2 t} \\
& =58 \mathrm{psi}(49 / 2 \mathrm{in}) /\left(2^{*} 0.307 \mathrm{in}\right) \\
& =2314.33 \mathrm{psi} \\
& =15.96 \mathrm{MPa}
\end{aligned}
$$




\section{Appendix B: Theoretical Calculation of Stress for Full Scale Prototype}

i. Spherical Cap Region:

The theoretical calculation for,

Hoop stress, $\quad S_{11}=\frac{P R}{2 t}$

Longitudinal stress, $\quad S_{22}=\frac{P R}{2 t}$

$$
\begin{aligned}
& =17(97.19) /\left(2^{*} 0.307\right) \\
& =2690.93 \mathrm{psi} \\
& =18.55 \mathrm{MPa}
\end{aligned}
$$

ii. Cylindrical Region:

The theoretical calculation for

$$
\text { Hoop stress, } \quad \begin{aligned}
S_{11} & =\frac{P R}{t} \\
& =17(97.19) /(0.307) \\
& =5381.86 \mathrm{psi} \\
& =37.11 \mathrm{MPa}
\end{aligned}
$$

The theoretical calculation for

$$
\text { Longitudinal stress, } \quad \begin{aligned}
S_{22} \quad & =\frac{P R}{2 t} \\
& =17(97.19) /\left(2^{*} 0.307\right) \\
& =2690.93 \mathrm{psi} \\
& =18.55 \mathrm{MPa}
\end{aligned}
$$




\section{Appendix C: Calculation of Equivalent Cross Sectional Area of Rope}

\section{Calculation of Equivalent Cross Sectional Area of Rope for Quarter Scale RTP}

Cross Sectional Area of Actual Rope and Fabric,

Assumed diameter of rope, $d_{a} \quad=1.0 \mathrm{in}$

Width of membrane, $W_{o} \quad=1.0$ in

Thickness of membrane, $t_{o} \quad=0.307$ in

Area of circular Rope, $d_{a} \quad=\pi(\mathrm{d} / 2)^{2}=\pi(1.0 / 2)^{2}=0.7854 \mathrm{in}^{2}$

Rope-representing Equivalent Area, $A=t_{1} * W_{o}=0.7854 \mathrm{in}^{2}$

Thickness of equivalent, $t_{1} \quad=0.7854 / 1.0=0.7854$ in

Total thickness of membrane, $T_{\text {total }}=t_{1}+t_{0}=0.7854+0.307=1.0924$ in

\section{Calculation of Equivalent Cross Sectional Area of Rope for Full Scale RTP}

Cross Sectional Area of Actual Rope and Fabric,

Assumed diameter of rope, $d_{a} \quad=2.0$ in

Width of membrane, $W_{o} \quad=2.0$ in

Thickness of membrane, $t_{o} \quad=0.307$ in

Area of circular Rope, $d_{a} \quad=\pi(\mathrm{d} / 2)^{2}=\pi(2 / 2)^{2}=3.142 \mathrm{in}^{2}$

Rope-representing Equivalent Area, $A=t_{1} * W_{o}=3.142 \mathrm{in}^{2}$

Thickness of equivalent, $t_{1} \quad=3.142 / 2=1.571$ in

Total thickness of membrane, $T_{\text {total }} \quad=t_{1}+t_{0}=1.571+0.307=1.878 \mathrm{in}$ 

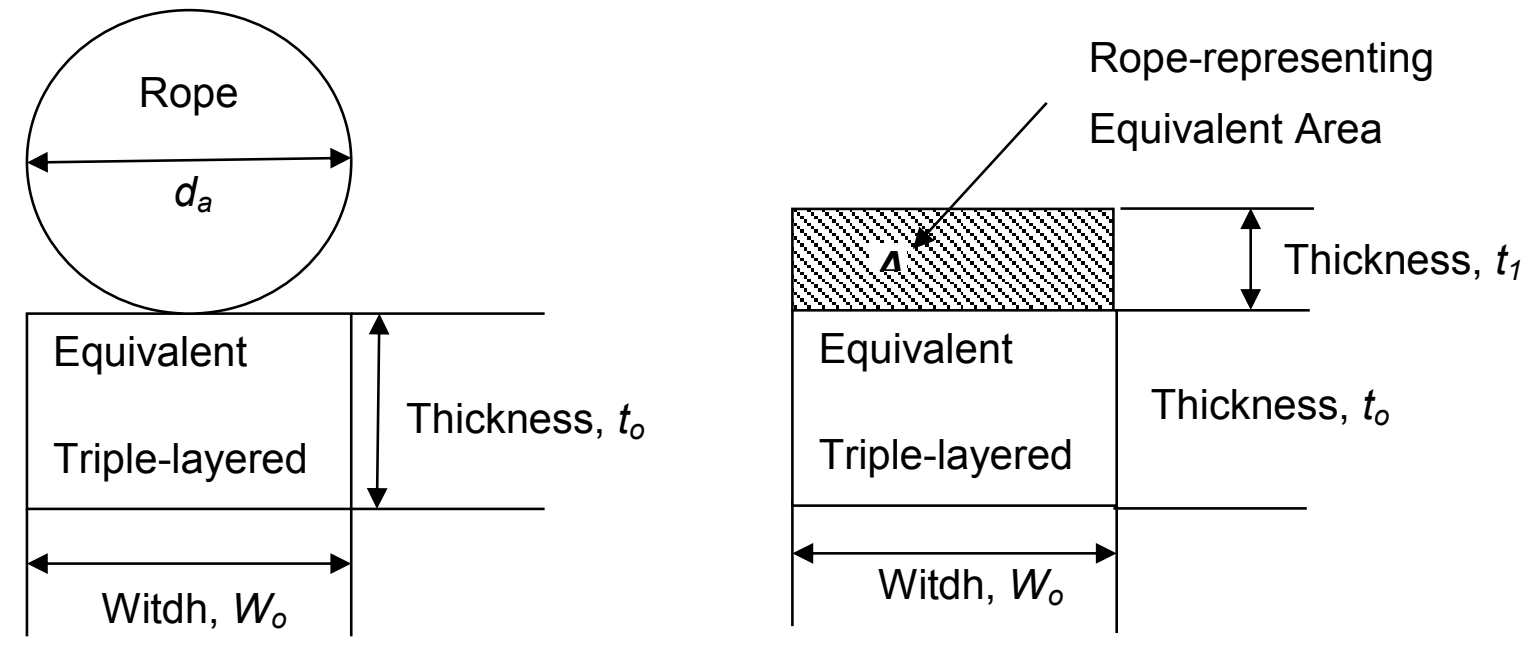


\section{Appendix D: Calculation of Elastic Force for a Connector Element.}

Width of tie, $w$

Thickness of tie, $t$

Initial length, $L_{0}$

The change of length of tie, $\delta$

Experimental Strain, $\varepsilon$

Experimental break strength, $F$

Elastic force (stiffness), $k$
$=0.35$ in

$=0.07 \mathrm{in}$

$=2.25$ inches

$=0.8$ inch

$=\frac{\delta}{L_{0}}=0.35$

$=175 \mathrm{lbf}=778.4387 \mathrm{~N}$

$=\frac{F}{\delta}$

$=\frac{175}{0.8}$

$=218.75 \mathrm{lbf} / \mathrm{in}=38308.99 \mathrm{~N} / \mathrm{m}$ 


\section{Appendix E: Calculation of Equivalent Density for Full-Scale Slippage Simulation.}

Volume within a constrained full-scale plug, $\mathrm{V}_{\text {plug }}$

Density of water, $\rho_{\text {water }}$

Density of air, $\rho_{\text {air }}$

Mass of water in a constrained full-scale plug, $m_{\text {water }}$

Volume of solid elements, $\mathrm{V}_{\text {se }}$

Equivalent density (water) of solid elements, $\rho_{\text {Eqwater }}$
$=7.6357 \mathrm{E} 6 \mathrm{in}^{3}$

$=0.036 \mathrm{lbm} / \mathrm{in}^{3}$

$=4.3 \mathrm{E}-5 \mathrm{lbm} / \mathrm{in}^{3}$

$=0.036 * 7.6357 \mathrm{E} 6$

$=274886 \mathrm{lbm}$

$=6.6 \mathrm{E} 6 \mathrm{in}^{3}$

$=274886 / 6.6 \mathrm{E} 6$

$=0.0416 \mathrm{lbm} / \mathrm{in}^{3}$

$=1.079 \mathrm{E}-4 \mathrm{lbf} \cdot \mathrm{sec}^{2} / \mathrm{in}^{4}$

Mass of air in a constrained full-scale plug, $m_{\text {air }}$

$$
\begin{aligned}
& =4.3 \mathrm{E}-5^{\star} 7.6 \mathrm{E} 6 \\
& =326.8 \mathrm{lbm} \\
& =2000+326.8 \\
& =2326.8 \mathrm{lbm} \\
& =70500 \mathrm{in}^{3} \\
& =2326.8 / 70500 \\
& =0.033 \mathrm{lbm} / \mathrm{in}^{3} \\
& =8.55 \mathrm{E}-5 \mathrm{lbf} . \mathrm{sec}^{2} / \mathrm{in}^{4}
\end{aligned}
$$

